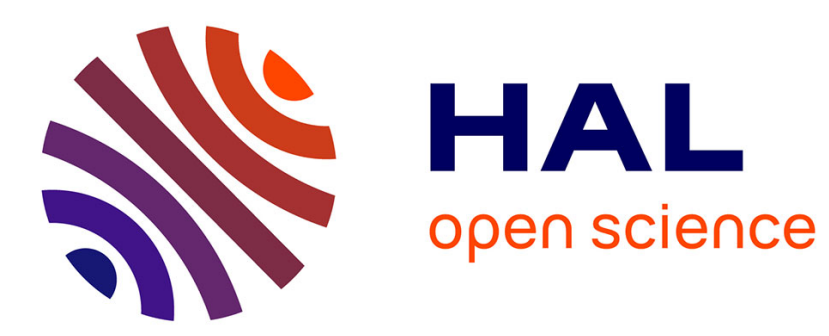

\title{
Sur le transfert des traces d'un groupe classique p-adique à un groupe linéaire tordu
}

Colette Moeglin, Jean-Loup Waldspurger

\section{To cite this version:}

Colette Moeglin, Jean-Loup Waldspurger. Sur le transfert des traces d'un groupe classique p-adique à un groupe linéaire tordu. 2005. hal-00014487

\section{HAL Id: hal-00014487 \\ https://hal.science/hal-00014487}

Preprint submitted on 25 Nov 2005

HAL is a multi-disciplinary open access archive for the deposit and dissemination of scientific research documents, whether they are published or not. The documents may come from teaching and research institutions in France or abroad, or from public or private research centers.
L'archive ouverte pluridisciplinaire HAL, est destinée au dépôt et à la diffusion de documents scientifiques de niveau recherche, publiés ou non, émanant des établissements d'enseignement et de recherche français ou étrangers, des laboratoires publics ou privés. 


\title{
Sur le transfert des traces d'un groupe classique p-adique à un groupe linéaire tordu.
}

\author{
C. Mœglin et J.-L. Waldspurger \\ Institut de Mathématiques de Jussieu \\ CNRS
}

Soit $F$ un corps local non archimédien et soit $N$ un entier. On note $\theta$ l'automorphisme de $G L(N, F)$ défini pour tout élément $g$ de $G L(N, F)$ par $\theta(g)=J^{t} g^{-1} J$ où $J$ est la matrice antidiagonale multiplié à gauche par la matrice diagonale $\left(1,-1, \cdots,(-1)^{N-2},(-1)^{N-1}\right)$. On note $\tilde{G}(N)$ le produit semidirect de $G L(N, F)$ par le groupe à 2 éléments engendré par $\theta$. Pour justifier notre travail, admettons momentanément que la conjecture de Ramanujan soit vérifiée pour les représentations automorphes cuspidales de $G L(N, \mathbb{A})$ où $\mathbb{A}$ est l'anneau des adèles d'un corps global admettant $F$ comme localisé en une place. Les composantes locales en cette place des formes automorphes de carré intégrable (et même des séries d'Eisenstein sur l'axe unitaire) sont des induites irréductibles de la forme $\sigma:=$ $\times_{(\rho, a, b)} S p(b, S t(a, \rho))$ où $(\rho, a, b)$ parcourt un ensemble de triplets où $\rho$ est une représentation cuspidale irréductible et unitaire d'un groupe $G L\left(d_{\rho}, F\right)$ (ce qui définiti $d_{\rho}$ ) et $a, b$ sont des entiers. La notation $S p(b, S t(a, \rho))$ est importante pour nous : pour la définir on utilise des induites ce qui suppose que nous avons fixé un parabolique minimal, pour nous les matrices triangulaires supérieures (pour fixer les idées), alors $S t(a, \rho)$ est la représentation de Steinberg de $G L\left(a d_{\rho}, F\right)$ basée sur $\rho$, c'est-à-dire l'unique sous-module irréductible de l'induite $\left.\rho\right|^{(a-1) / 2} \times \cdots \times \rho \|^{-(a-1) / 2}$ et $S p(b, S t(a, \rho))$ est l'unique quotient irréductible de l'induite pour $G L\left(a b d_{\rho}, F\right)$ :

$$
\left.S t(a, \rho)\right|^{(b-1) / 2} \times \cdots \times\left. S t(a, \rho)\right|^{-(b-1) / 2} .
$$

C'est aussi l'unique sous-module irréductible de l'induite

$$
S t(a, \rho)||^{-(b-1) / 2} \times \cdots \times S t(a, \rho)||^{(b-1) / 2} .
$$

On remarque aussi que $\rho$ est $\theta$-invariante si et seulement si $\rho$ est autoduale. Si l'ensemble des représentations $\rho$ qui interviennent dans ce produit est formé de représentations dont la classe d'isomorphie est invariante sous l'action de $\theta$ (on dira représentations $\theta$-invariantes), l'induite $\sigma$ est elle aussi $\theta$-invariante et on peut donc la prolonger en une action de $\tilde{G}(N)$; il y a évidemment un choix, qu'il faudra préciser. D'un point de vue global, un choix pour l'action de $\theta$ s'impose assez naturellement : celui qui vient de la classification de Langlands des représentations. C'est celui qui est suggéré par Arthur. D'un point de vue local, ce n'est pas le plus simple et il n'a rien de canonique. Dans ce travail, on étudiera les différents choix possible, on calculera les signes qui interviennent quand on change de choix et on montrera l'influence de ces signes sur le transfert.

Mais le but principal de ce travail est de ramener le calcul de la trace $\operatorname{tr} \sigma(g, \theta)$ pour tout $g \in$ $G L(N, F)$ au calcul de la trace pour des représentations tempérées. Pour cela, nous n'avons besoin d'aucune hypothèse.

Ce cas a été traité sous certaines hypothèses (en particulier un lemme fondamental) en [27] et sous ces mêmes hypothèses on obtient donc un transfert assez général. Le but ultime est de décrire les paquets d'Arthur pour les groupes classiques; ici on règle donc la combinatoire liée à la distribution stable associée au paquet. Il restera à régler la combinatoire liée aux propriétés endoscopiques, et on voit clairement comment faire. Soyons plus précis.

Arthur [1] [6] a remarqué que si $N$ est pair le groupe $H_{N}:=S O(N+1, F)$ (forme quasidéployée) est un groupe endoscopique "stable" pour la forme tordue $\tilde{G}(N)$ alors que si $N$ est impair, la même 
assertion est vraie pour $H_{N}:=S p(2 N, F)$. Ainsi si $\operatorname{tr}(\sigma(g, \theta))$ est stable, cette distribution doit être le tranfert d'une distribution stable sur l'un de ces groupes; quand cette distribution n'est pas stable d'atures groupes endoscopiques elliptiques remplace $H_{N}$ (cf [6] par. 30. L'idée serait de calculer $\operatorname{tr}(\sigma(g, \theta))$ dans le groupe de Grothendieck des représentations lisses de $\tilde{G}(N)$ avec comme base des induites de représentations tempérées, en fait ce n'est pas tout à fait comme cela que l'on procède. On interprétera la représentation $\sigma$ à l'aide des morphismes de $W_{F} \times S L(2, \mathbb{C}) \times S L(2, \mathbb{C})$ dans $G L(N, \mathbb{C})$ dont la restriction à $W_{F}$ est continue et bornée. Et les représentations tempérées qui doivent alors intervenir sont celles qui sont associées au morphisme $\psi \circ \Delta$ où $\Delta$ est le plongement diagonal de $S L(2, \mathbb{C})$ pour tous les sous-groupes de Levi de $G L(N, F)$ tel que le groupe dual contienne un conjugué de $\psi \circ \Delta$. On oublie la notation $\sigma$ et on note $\pi(\psi)$ la représentation associée à un tel morphisme $\psi$. On veut étudier les $\operatorname{tr} \pi(\psi)(g, \theta)$ quand l'image de $\psi$ est simplement $\theta$-invariante.

Le cas difficile est celui où $\psi \circ \Delta$ est discret c'est-à-dire défini une représentation semi-simple sans multiplicités de $W_{F} \times S L(2, \mathbb{C})$; on dit alors que $\psi$ est de restriction discrète à la diagonale. On ramène le cas général (cf 2.4) à ce cas, en utilisant le Jacquet ce qui est une opération qui nécessite de commencer par augmenter le rang du groupe mais cette opération est très compatible au transfert (cf. 4.2).

Supposons donc que $\psi \circ \Delta$ soit discret; dans ce cas, le centralisateur de $\psi$ et celui de $\psi \circ \Delta$ sont des produits de groupes $G L(1, \mathbb{C})$ et celui de $\psi$ est inclus dans celui de $\psi \circ \Delta$. On dit que $\psi$ est élémentaire si cette inclusion est une bijection; en termes simples cela veut dire que $\psi$ en tant que représentation de $W_{F} \times S L(2, \mathbb{C}) \times S L(2, \mathbb{C})$ est somme de représentations irréductibles dont chacune est triviale sur au moins l'une des copies de $S L(2, \mathbb{C})$; la représentation de $G L(n, F)$ alors associée à $\psi$ est une induite irréductible de représentations de Steinberg et de représentations de Speh (les représentations de Speh sont les images par l'involution de Zelevinsky des représentations de Steinberg). Ce cas se traite en réalisant la représentation associée à $\psi$ comme cohomologie d'un complexe provenant de la représentation tempérée associée à $\psi \circ \Delta$; c'est une réminiscence des constructions d'Aubert [7]. Ce complexe est muni d'une action de $\theta$ et il faut la comparer à l'action de $\theta$ mise a priori. Ceci est fait en 3. Le travail combinatoire de toute la première partie du papier est de ramener le cas des $\psi$ généraux de restriction discrète à la diagonale au cas élémentaire; on réalise la représentation associée à $\psi$ dans le groupe de Grothendieck avec comme base des induites de représentations associées à des morphismes élémentaires. Nous allons essayer de donner ici une idée de la formule; on fixe une sous-représentation irréductible de la représentation de $W_{F} \times S L(2, \mathbb{C}) \times S L(2, \mathbb{C})$. Une telle représentation est le produit tensorielle de 3 représentaions irréductibles, l'une de $W_{F}$ que nous appelons $\rho$ et deux de $S L(2, \mathbb{C})$ qui sont connues dès que l'on a leurs dimensions, dimensions que l'on note $a, b$ respectivement. Ainsi $\psi$ est la somme d'une représentation associée à un morphisme du même type $\psi^{\prime}$ et de cette représentation que nous écrivons $\pi(\rho, a, b)$; comme $\psi$ n'est pas élémentaire, on suppose que $\inf (a, b)>1$ et pour n'utiliser dans cette introduction que la représentation de Steinberg, on suppose ici que $a \geq b$. Grâce à la correspondance de Langlands locale [9], [10], on identifie $\rho$ à une représentation cuspidale d'un groupe $G L\left(d_{\rho}, F\right)$ (ce qui définit $d_{\rho}$ ). Dans le groupe de Grothendieck on définit a priori la représentation suivante :

$$
\begin{gathered}
\oplus_{c \in] b, a]}(-1)^{a-c} S t(c, \rho)||^{(a-b-c+1) / 2} \times \pi_{c} \times S t(c, \rho)||^{-(a-b-c+1) / 2} \\
\oplus(-1)^{[b / 2]} S t(a-b+1, \rho) \times \pi(\rho, a+1, b-1) \times \pi\left(\psi^{\prime}\right),
\end{gathered}
$$

où $\pi_{c}$, pour $c=a$ vaut $\pi(\rho, a, b-2) \times \pi\left(\psi^{\prime}\right)$ (si $\left.b=2, \pi_{a}=\pi\left(\psi^{\prime}\right)\right)$, est la représentation 0 si $b=2$ et $c<a$ et sinon est un module de Jacquet convenable de la représentation $\pi(\rho, a+2, b-2) \times \pi\left(\psi^{\prime}\right)$ qui dépend de $c$ évidemment; on renvoie au texte pour une définition précise, le point important est que inf $(a+2, b-2)=b-2<b$ et de même inf $(a+1, b-1)=b-1<b$. Chacune des représentations écrites dans cette combinaison linéaire a une action de $\theta$ que l'on précise de façon ad hoc de façon à obtenir le résultat : cet élément du groupe de Grothendieck de $\tilde{G}(N)$ vérifie pour tout élément $g \in G L(n, F)$ suffisamment régulier $\operatorname{tr} \tilde{\pi}(\psi)(g, \theta)=\operatorname{tr} \pi(\psi)(g, \theta)$. On conclut en faisant une récurrence sur $I_{\psi}:=\sum_{\left(\rho^{\prime}, a^{\prime}, b^{\prime}\right)}$ inf $\left(a^{\prime}, b^{\prime}\right)$ où $\left(\rho^{\prime}, a^{\prime}, b^{\prime}\right)$ parcourt l'ensemble des représentations irréductibles incluses dans $\psi$. Revenons sur le problème de l'action de $\theta$; on définit a priori une action de $\theta$ sur toutes les représentations associées à des morphismes du type de $\psi$. Avec les notations ci- 
dessus, on aura canoniquement une action sur chaque représentation $\pi_{c}$ qui se prolonge canoniquement en une action sur l'induite correspondant à $c$ ci-dessus. Toutes ces actions provenant canoniquement d'une action mise sur $\pi(\rho, a+2, b-2) \times \pi\left(\psi^{\prime}\right)$, il est naturel qu'elles se combinent bien. Mais comme il y a encore le terme $S t(a-b+1, \rho) \times \pi(\rho, a+1, b-1) \times \pi\left(\psi^{\prime}\right)$ dans la combinaison linéaire, il faut que l'action mise sur $\pi(\rho, a+2, b-2) \times \pi\left(\psi^{\prime}\right)$ soit aussi liée à celle mise sur $\operatorname{St}(a-b+1, \rho) \times \pi(\rho, a+1, b-1) \times \pi\left(\psi^{\prime}\right)$. $\mathrm{Si}$ on prend l'action qui vient des classifications de Langlands (cf. 5.1), ici, cela fonctionnerait bien car on a supposé que $a \geq b$; mais dans le cas inverse, où $b \geq a$ et où les Steinberg sont remplacés par les "Speh" , cela ne fonctionnerait plus, il faudrait introduire un signe sur l'action de $\theta$. Plutôt que de mettre des signes, on a choisi de définir sur les représentations $\pi(\psi)$ une action de $\theta$ qui est "indépendante" des signes $a^{\prime}-b^{\prime}$ (où $\left(\rho^{\prime}, a^{\prime}, b^{\prime}\right.$ ) parcourt comme ci-dessus l'ensemble des sousreprésentations irréductibles incluses dans $\psi$ ). Et dans la partie 5, on étudie ces signes. D'un point de vue local il n'y a de toute façon aucune raison de privilégier la classification de Langlands à celle de Zelevinsky. Appelons normalisation à la Whittaker, l'action de $\theta$ obtenue en utilisant la normalisation de Langlands ; un peu plus précisément la classification de Langlands fournit une induite (nécessairement $\theta$-invariante) tel que $\pi(\psi)$ soit l'unique quotient irréductible (dans tout le travail on utilise sous-module et non quotient en dualisant convenablement). L'induite a un modèle de Whittaker pour tout choix de caractère additif de $F$. On fait un tel choix de caractère additif et avec ce choix on construit un caractère $\chi$ du sous-groupe unipotent supérieur de $G L(n, F)$ invariant sous l'action de $\theta$; pour tout représentation $I$ de $G L(n, F)$, munie d'une action de $\theta$, l'espace des coinvariants pour ce sous-groupe unipotent et le caractère $\chi$ hérite d'une action de $\theta$. Si l'espace des coinvariants est de dimension 1, ce qui est le cas ci-dessus, on a ainsi un moyen de normaliser l'action de $\theta$ en demandant que l'action induite soit l'action triviale. C'est ce que l'on appelle la normalisation à la Whittaker. On vérifiera que ce choix est indépendant du caractère additif fixé quand $\psi$ se factorise par le $L$-groupe de $H_{N}$. On note $\theta_{W}(\psi)$ l'action ainsi définie de $\theta$. Quand on utilise la classification de Zelevinsky, on réalise $\pi(\psi)$ comme unique sous-module irréductible d'une induite $\theta$-invariante ; ici l'induite n'a pas de modèle de Whittaker en général, il faut donc commencer par mettre une action de $\theta$ sur l'induite puis la restreindre (c'est expliqué en 5.3). On note $\theta_{U}(\psi)$ cette normalisation et on note $\theta(\psi)$ la normalisation "ad hoc", celle qui fait "marcher" le résultat ci-dessus. Il n'y a plus besoin de supposer ici que $\psi$ est de restriction discrète à la diagonale. On calcule explicitement tous les signes $\sigma_{W}(\psi):=\theta(\psi) / \theta_{W}(\psi), \sigma_{U}(\psi):=\theta(\psi) / \theta_{U}(\psi) \sigma_{\emptyset}(\psi):=\theta_{W}(\psi) / \theta_{U}(\psi)$; ces signes ont une jolie interprétation relativement au transfert endoscopique. Ici on se limite au cas où $\psi$ se factorise par le $L$-groupe de $H_{N}$, cas où $\pi(\psi) \circ \theta$ est stable d'après [6]. On montre alors qu'il existe un caractère $\epsilon_{W}(\psi), \epsilon_{U}(\psi)$ et $\epsilon_{\emptyset}(\psi)$ du centralisateur de $\psi$ dans le $L$-groupe de $H_{N}$ dont la restriction au centre de ce $L$-groupe est triviale et tel que en notant $z_{2}$ l'image par $\psi$ de l'élément non trivial de la 2e copie de $S L(2, \mathbb{C})$, le signe $\sigma_{?}(\psi)$ soit la valeur du caractère $\epsilon_{?}(\psi)$ sur $z_{2}$.

Supposons encore que $\psi$ se factorise par le $L$-groupe de $H_{N}$; pour motiver notre article on rappelle en 4.6 comment on a associé à tout caractère $\epsilon \operatorname{de} \operatorname{Cent}(\psi)$ dans ce $L$-groupe une représentation $\pi(\psi, \epsilon)$ ou (parfois) 0 que l'on note $\pi^{H}(\psi, \epsilon)$; on a étudié ces représentations sous certaines hypothèses mais ici seule la construction nous importe. Le transfert que l'on obtient dans les cas où on le connaît pour les représentations tempérées (cf. 4.4 pour des hypothèses précises) est de la forme :

$$
\sum_{\epsilon} \epsilon\left(z_{2}\right) \operatorname{tr} \pi^{H}(\psi, \epsilon)=\operatorname{tr} \pi(\psi) \circ \theta
$$

où $\epsilon$ parcourt l'ensemble des caractères du centralisateur de $\psi$ dans le $L$-groupe de $H_{N}$ triviaux sur le centre de ce $L$-groupe. Et avec la propriété donnée une telle formule ne doit être montrée que pour l'une des actions de $\theta$, on l'obtient ensuite pour les autres en changement l'application $\epsilon \mapsto \pi^{H}(\psi, \epsilon)$ en $\epsilon \mapsto \pi(\psi, \epsilon$ ? $\epsilon)$. Cette formule est heureusement analogue à celle de [6] 30.1 qui fait suite à [2], [3], [4], [5]. Notre point de vue est une description explicite des représentations qui interviennent. On explique d'ailleurs en 4.5 comment le transfert obtenu par Arthur est un point départ pour nos constructions et ce qu'il entraîne des hypothèses dont nous avons besoin pour aller au bout des descriptions ; on pousse ce point de vue en [17] pour avoir toutes les hypothèses dont nous avons besoin pour une description combinatoire mais cela sort du cadre de ce travail limité à $G L(N, F)$ tordu. 


\section{Définitions et notations générales}

\section{$1.1 \quad$ Notations pour les intervalles}

A de nombreuses reprises dans cet article des sommes ou des produits sont indexés par des élément $\ell$ dans un segment; on a pris quelques conventions sur cette notations. Pour nous un segment est la donnée de 2 nombres réels $x, y$ tel que $x-y \in \mathbb{Z}$ (en général $x, y$ seront des demi-entiers non nécessairement entiers) et la notation $\ell \in[x, y]$ est l'ensemble des éléments $\ell$ de la forme $x, x-$ $1, \cdots, y+1, y$ si $x \geq y$ et $x, x+1, \cdots, y-1, y$ si $x \leq y$.

\subsection{Action de $\theta$ sur une représentation cuspidale autoduale}

Soit $\rho$ une représentation cuspidale irréductible de $G L\left(d_{\rho}, F\right)$ (ce qui définit $d_{\rho}$ ). On suppose que $\rho$ est invariante par conjugaison sous $\theta$ (action définie ci-dessus quand on fait $n=d_{\rho}$ ). On fixe un espace de la représentation $\rho$ muni d'un automorphisme $N_{\rho, \theta}$ tel que $N_{\rho, \theta}$ entrelace $\rho$ et $\rho \circ \theta$ et on suppose que $N_{\rho, \theta}^{2}=1$. On considère $\rho$ comme une représentation du produit semi-direct $\tilde{G}\left(d_{\rho}\right)$ où $\theta$ agit par l'opérateur $N_{\rho, \theta}$. On a fait là un choix qui dure dans tout le texte; une façon de normaliser est de normaliser à la Whittaker (comme expliqué dans l'inroduction), ce qui suppose que l'on a fixé un caractère additif du corps de base; on vérifiera qu'un tel choix n'influe pas sur le transfert (cf. 4.1).

\subsection{Action de $\theta$ sur des induites}

De façon générale, soit $\sigma$ une représentation de $\tilde{G}\left(d_{\sigma}\right)$ (ce qui définit $d_{\sigma}$ ) et soit $\tau$ une représentation de $G L\left(d_{\tau}, F\right)$ (ce qui définit $d_{\tau}$ ). On note ${ }^{\theta} \tau$ l'image de $\tau$ par l'automorphisme induit par $\theta$ et soit $\tau^{\prime}$ une représentation de $G L\left(d_{\tau}, F\right)$ isomorphe à ${ }^{\theta} \tau$. Soit $A$ un automorphisme de l'espace, $V_{\tau}$, de $\tau$ sur l'espace $V_{\tau^{\prime}}$, de $\tau^{\prime}$ tel que pour tout $v$ dans l'espace de $\tau$ et tout $g \in G L\left(d_{\tau}, F\right)$, on ait $\tau^{\prime}(g) A(v)=A(\tau(\theta(g)) v)$. On note $V_{\sigma}$ l'espace de $\sigma$ et $\theta_{\sigma}$ l'action de $\theta$ sur cet espace. On pose pour tout $\phi$ dans l'induite de $\tau \otimes \sigma \otimes \tau^{\prime}$ et tout $g \in G L\left(d_{\sigma}+2 d_{\tau}, F\right)$ :

$$
\theta \phi(g):=\left(A^{-1} \otimes \theta_{\sigma} \otimes A\right) \iota(\phi(\theta(g)))
$$

où $\iota$ est l'isomorphisme de $V_{\tau} \otimes V_{\sigma} \otimes V_{\tau^{\prime}}$ sur $V_{\tau^{\prime}} \otimes V_{\sigma} \otimes V_{\tau}$ qui échange les termes extrêmes. On vérifie que cela définit une action de $\theta$ sur l'induite $\tau \times \sigma \times \tau^{\prime}$; en effet soit $m_{1} \in G l\left(d_{\tau}, F\right), m_{0} \in G L\left(d_{\sigma}, F\right)$ et $m_{2} \in G L\left(d_{\tau}, F\right)$. On considère $m:=\left(m_{1}, m_{0}, m_{2}\right)$ comme un élément de $G L\left(d_{\sigma}+2 d_{\tau}, F\right)$. Pour $g$ comme ci-dessus, on a $\theta(m g)=\left(\theta\left(m_{2}\right), \theta\left(m_{0}\right), \theta\left(m_{1}\right)\right) \theta(g)$ et l'assertion s'en déduit. Ainsi l'induite $\tau \times \sigma \times \tau^{\prime}$ est une représentation de $\tilde{G}\left(d_{\sigma}+2 d_{\tau}\right)$ et cette action dépend du choix de $A$ à ceci près qu'elle est indépendante de la multiplication de $A$ par un scalaire; en particulier si $\tau$ est irréductible, elle ne dépend pas du choix de $A$. On généralise cette construction en remplaçant $\tau$ par une induite $\tau_{1} \times \cdots \times \tau_{\ell}$, où chaque $\tau_{i}$ est irréductible; on voit clairement comment prolonger naturellement l'action de $\theta_{\sigma}$ à l'induite $\tau_{1} \times \cdots \times \tau_{\ell} \times \sigma \times{ }^{\theta} \tau_{\ell} \times \cdots \times{ }^{\theta} \tau_{1}$ de façon indépendante de tout choix de morphisme, $A_{1}, \cdots, A_{\ell}$ entrelaçant, pour $i=1, \cdots, \ell,{ }^{\theta} \tau_{i}$ et $\tau_{i} \circ \theta$.

Pour $\rho$ fixé et $a$ un entier, on note $S t(a, \rho)$ la représentation de Steinberg qui est l'unique sousreprésentation irréductible de l'induite $\sigma_{S t, a, \rho}:=\left.\rho\right|^{(a-1) / 2} \times \cdots \times\left.\rho\right|^{-(a-1) / 2}$ et pour $b$ un entier, on note $S p(b, \rho)$ la représentation de Speh qui est l'unique sous-représentation irréductible de l'induite $\sigma_{S p, b, \rho}:=\rho||^{-(b-1) / 2} \times \cdots \times\left.\rho\right|^{(b-1) / 2}$.

\subsection{Notations relatives aux modules de Jacquet}

Soit $\pi$ une représentation de $\tilde{G}(N)$ de longueur finie et soit $\rho$ une représentation irréductible cuspidale et autoduale de $G L\left(d_{\rho}, F\right)$. On considère le parabolique standard de $G L(n, F)$ de Levi $G L\left(d_{\rho}, F\right) \times G L\left(N-2 d_{\rho}, F\right) \times G L\left(d_{\rho}, F\right)$; on remarque qu'il est $\theta$-invariante. On considère le module de Jacquet de $\pi$ le long du radical unipotent de ce parabolique. On considère ce module de Jacquet dans le groupe de Grothendieck des représentations du Levi . On fixe $x \in \mathbb{R}$ et on projette cet élément semi-simplifié sur le support cuspidal $\left.\rho\right|^{x}$ par l'action du premier facteur $G L\left(d_{\rho}, F\right)$ et sur le support 
cuspidal $\rho||^{-x}$ pour le deuxième facteur $G L\left(d_{\rho}, F\right)$ (le dernier dans l'écriture du Levi) ; cette projection semi-simplifiée est une somme de représentations irréductibles $\left.\left.\rho\right|^{x} \otimes \sigma \otimes \rho\right|^{-x}$ où $\sigma$ parcourt un ensemble de représentations irréductibles de $G L\left(N-2 d_{\rho}, F\right)$. On note $J a c_{x}^{\theta}(\pi)$ l'élément du groupe de Grothendieck de $G L\left(N-2 d_{\rho}, F\right)$ qui est la somme de ces représentations $\sigma$. Comme $\pi$ a une action de $\theta$, on obtient une action de $\theta$ sur le module de Jacquet tout entier puis sur sa projection sur la composante correspond à l'action de $\left.\rho\right|^{x}$ pour le premier facteur $G L\left(d_{\rho}, F\right)$ et $\left.\rho\right|^{-x}$ pour le dernier facteur. On obtient donc une action du produit semi-direct, $\tilde{M}$, de $G L\left(d_{\rho}, F\right) \times G L\left(n-2 d_{\rho}, F\right) \times G L\left(d_{\rho}, F\right)$ par le groupe $\{1, \theta\}$. On a défini $\tilde{G}\left(n-2 d_{\rho}\right)$ définition que l'on prolonge au cas où $n=2 d_{\rho}$ en considérant que dans ce cas le groupe est $\{1, \theta\}$. Et $\tilde{G}\left(n-2 d_{\rho}\right)$ se plonge naturellement dans $\tilde{M}$. Supposons d'abord que la projection considérée du module de Jacquet de $\pi$ est semi-simple en tant que représentation de $\tilde{M}$. Elle se réalise alors dans un espace $V \otimes W \otimes V$ où $V$ est un espace pour la représentation $\rho$ et $W$ est une représentation semi-simple, $\sigma_{W}$, de $G L\left(n-2 d_{\rho}, F\right)$. On fixe un opérateur $A$ de $A u t(V)$ qui entrelace $\rho$ et ${ }^{\theta} \rho$ et on note $\iota$ l'échange des 2 facteurs $V$. Alors $D:=\left(A \otimes i d_{W} \otimes A^{-1}\right) \circ \iota \circ \theta$ est un opérateur de $V \otimes W \otimes V$ qui vérifie pour tout $m, m^{\prime} \in G L\left(d_{\rho}, F\right)$ et $h \in G L\left(n-2 d_{\rho}, F\right)$ :

$$
D \circ\left(\rho||^{x} \otimes \sigma_{W} \otimes \rho||^{-x}\right)\left(m, h, m^{\prime}\right)=\left(\rho||^{x} \otimes \sigma_{W} \otimes \rho||^{-x}\right)\left(m, \theta(h), m^{\prime}\right) \circ D .
$$

Par irréductibilité de $\rho, D$ est donc de la forme, $i d \otimes D_{W} \otimes i d$ et $D_{W}$ est indépendant du choix de $A$ fait ci-dessus. Ainsi $W$ est muni d'une action de $\tilde{G}\left(n-2 d_{\rho}\right)$ en faisant agir $\theta$ via $D_{W}$. En filtrant convenablement le module de Jacquet de $\pi$, par cette méthode, on munit $\operatorname{Jac}^{\theta}(\pi)$ d'une action semisimple de $\tilde{G}\left(n-2 d_{\rho}\right)$ qui ne dépend que de l'action de $\theta$ sur $\pi$. Ceci permet de considérer $J_{a c}^{\theta}(\pi)$ comme un élément du groupe de Grothendieck de $\tilde{G}\left(N-2 d_{\rho}\right)$ et c'est ce que nous ferons. On remarque que cette définition n'utilise que l'image de $\pi$ dans le groupe de Grothendieck de $\tilde{G}(N)$. Quant au lieu d'avoir un seul réel $x$, on en a une collection ordonnée, $x_{1}, \cdots, x_{v}$, on itère les définitions en posant :

$$
J a c_{x_{1}, \cdots, x_{v}}^{\theta}(\pi):=J a c_{x_{v}}^{\theta} \cdots J a c_{x_{1}}^{\theta}(\pi) .
$$

Dans ce papier on reprend aussi la notation $J a c_{x}(\pi)$ où ici $\rho, x$ sont comme ci-dessus, $\pi$ est simplement une représentation de longueur finie de $G L(N, F)$ et on considère le module de Jacquet de $\pi$ pour le parabolique standard de Levi $G L\left(d_{\rho}, F\right) \times G L\left(N-d_{\rho}, F\right)$ avec sa projection sur le support cuspidal $\rho \mid{ }^{x}$ pour l'action du premier facteur. La propriété que nous utiliserons est la suivante : soit $x, y$ tels que $x-y \neq \pm 1$ alors $J a c_{x, y}(\pi)=J a c_{y, x}(\pi)$ égalité dans le groupe de Grothendieck. En effet pour calculer $J a c_{x, y}(\pi)$ on commence par calculer le module de Jacquet pour le parabolique $G L\left(2 d_{\rho}, F\right) \times$ $G L\left(N-2 d_{\rho}, F\right)$; puis on projette sur le support cuspidal $\left.\rho\right|^{x},\left.\rho\right|^{y}$ pour l'action du premier facteur. Toute représentation irréductible de $G L\left(2 d_{\rho}, F\right)$ ayant ce support cuspidal est l'induite irréductible $\left.\rho\right|^{x} \times\left.\rho\right|^{y}$. D'où la symétrie entre $x$ et $y$. Cette formule se généralise en $J a c_{x, y}^{\theta}=J a c_{y, x}^{\theta}$ sous les mêmes hypothèses (implicitement ici $N \geq 4 d_{\rho}$ ).

\subsection{Notation pour le socle d'une induite}

Soient $m \in \mathbb{N}$ et $m_{1}, \cdots, m_{r}$ une collection d'entiers tels que $m=\sum_{i \in[1, r]} m_{i}$. Pour $i \in[1, r]$ soit $\sigma_{i}$ une représentation de $G L\left(m_{i}, F\right)$. On considère

$$
\sigma:=\times_{i \in[1, r]} \sigma_{i}
$$

la représentation induite de $G L(m, F)$. Le socle d'une représentation est la somme de ses sous-modules irréductibles et on note ce socle

$$
<\sigma_{1}, \cdots, \sigma_{r}>\text {. }
$$

On n'utilisera cette définition que dans des cas où le socle est irréductible et essentiellement dans le cas suivant : $\rho$ est une représentation cuspidale fixé, $[x, y]$ est un intervalle au sens défini ci-dessus et on prend pour $r:=|(x-y+1)|$ et pour $\sigma_{i}:=\left.\rho\right|^{x-i+1}$ si $x \geq y$ et $\left.\rho\right|^{x+i-1}$ si $y \geq x$. Le socle est alors noté $<\rho||^{x}, \cdots, \rho||^{y}>$. 


\subsection{Notation pour les ||$^{s}$ et les opérateurs d'entrelacement standard}

Soit $M$ un sous-groupe de Levi de $G L(N, F)$ et $\sigma$ une représentation irréductible de $M$; supposons que $M$ soit maximal, il s'écrit alors comme produit $G L\left(N_{1}, F\right) \times G L\left(N_{2}, F\right)$ et $\sigma$ est un produit tensoriel $\sigma_{1} \otimes \sigma_{2}$. Pour $s \in \mathbb{C}$, on définit l'induite pour le parabolique standard :

$$
\sigma_{1}||^{s} \times \sigma_{2}
$$

où ||$^{s}$ est un raccourci pour $\left|\operatorname{det}_{G L\left(N_{1}, F\right)}\right|^{s}$. On dispose alors des opérateurs d'entrelacement standard

$$
M(s):=\sigma_{1}||^{s} \times \sigma_{2} \rightarrow \sigma_{2} \times \sigma_{1}||^{s}
$$

ces opérateurs sont obtenus par intégration pour $s$ tel que $\operatorname{Re} s>0$ puis par prolongement méromorphe. Ils n'ont pas de raison d'être holomorphe en $s=0$ mais on peut toujours trouver une fonction méromorphe de $s, r(s)$ tel que l'opérateur $r(s) M(s)$ soit holomorphe en $s=0$ et que sa valeur en $s=0$ donne un opérateur non nul.

Cette définition d'opérateur d'entrelacement standard, se généralise à tout sous-groupe de Levi.

\subsection{Action de $\theta$ sur $S p(b, \rho)$ et $S t(a, \rho)$}

On suppose que $\rho \simeq{ }^{\theta} \rho$ et on fixe $N_{\theta, \rho}$ un isomorphisme de $\rho$ avec ${ }^{\theta} \rho$. Pour tout réel $x, N_{\theta, \rho}$ entrelace naturellement la représentation $\rho||^{x}$ avec ${ }^{\theta} \rho||^{-x}$. Ainsi si $a$ est pair, la représentation induite $\sigma_{S t, a, \rho}$ est munie d'une structure de $\tilde{G}\left(a d_{\rho}\right)$ module en itérant la construction faite ci-dessus pour une induite en commençant avec $\sigma$ la représentation triviale. Si $a$ est impair, on fait la même construction mais en partant de $\sigma=\rho$. Dans le cas $a$ pair, la structure ne dépend pas du choix de $N_{\theta, \rho}$ par contre elle en dépend dans le cas impair. On peut évidemment faire la même chose pour $\sigma_{S p, b, \rho}$. Par restriction, on en déduit une structure de $\tilde{G}\left(a d_{\rho}\right)$-module sur $S t(a, \rho)$ et une structure de $\tilde{G}\left(b d_{\rho}\right)$-module sur $S p(b, \rho)$.

\subsection{Définition de l'action de $\theta$ sur $S p(b, S t(a, \rho))$}

On généralise ces définitions, en définissant pour $a, b$ des entiers $S p(b, S t(a, \rho))$ comme l'unique sous-module irréductible de l'induite :

$$
S t(a, \rho)||^{-(b-1) / 2} \times \cdots \times S t(a, \rho)||^{(b-1) / 2} .
$$

On sait ou on vérifie facilement que cette définition a une forme de symétrie en $a, b$ ou plutôt entre $S p$ et $S t$ dans la mesure où $S p(b, S t(a, \rho))$ est aussi l'unique sous-module irréductible de l'induite :

$$
\left.S p(b, \rho)\right|^{(a-1) / 2} \times \cdots \times\left. S p(b, \rho)\right|^{-(a-1) / 2} .
$$

Comme nous venons de munir $S t(a, \rho)$ d'une action de $\theta$, on en déduit une action de $\theta$ sur (1) et par restriction une action de $\theta$ sur $S p(b, S t(a, \rho))$ que l'on note momentanément $\theta_{S t}$. On peut faire la même chose pour (2) en utilisant l'action de $\theta$ sur $S p(b, \rho)$ ce qui donne une action de $\theta$ sur $S p(b, S t(a, \rho))$ que l'on note $\theta_{S p}$. Heureusement, ces actions sont les mêmes comme nous allons le vérifier.

En effet, si inf $(a, b)=1$, c'est exactement la définition : pour clarifier supposons que inf $(a, b)=a$, c'est-à-dire $a=1$, alors (1) n'est autre que $\sigma_{S p, b, \rho}$ et (2) est $S p(b, \rho)$. On a bien défini l'action de $\theta$ sur $S p(b, \rho)$ par restriction de celle sur $\sigma_{S p(b, \rho)}$.

On suppose donc maintenant que inf $(a, b)>1$ et on montre l'assertion par induction en l'admettant pour $S p\left(b^{\prime}, S t\left(a^{\prime}, \rho\right)\right)$ pour tout couple $\left(a^{\prime}, b^{\prime}\right)$ tel que $a+b>a^{\prime}+b^{\prime}$; dans ce qui suit si $a=2$ tout terme écrit ci-dessous contenant $a-2$ est la représentation triviale de $G L(0, F)=\{1\}$ et on prend la même convention si $b=2$. L'induite (1) contient la représentation

$$
S t(a, \rho)||^{-(b-1) / 2} \times S p(b-2, S t(a, \rho)) \times\left. S t(a, \rho)\right|^{(b-1) / 2} .
$$

En remplaçant $S t(a, \rho)$ par sa définition, on vérifie que (3) est un sous-module de l'induite :

$$
\rho||^{(a-b) / 2} \times \cdots \times \rho||^{-(a+b) / 2+1} \times S p(b-2, S t(a, \rho)) \times \rho||^{(a+b) / 2-1} \times \cdots \times \rho||^{-(a-b) / 2} .
$$


Sur (4), on a une action de $\theta$ en tant qu'induite mais il n'est pas clair a priori que l'action de $\theta$ sur (3) est la restriction de cette action sur (4). Toutefois c'est vrai car l'action sur (3) ne dépend pas du choix de $A$ l'opérateur qui identifie ${ }^{\theta}\left(S t(a, \rho)||^{-(b-1) / 2}\right)$ avec $S t(a, \rho)^{(b-1) / 2}$. On peut donc prendre pour $A$ la restriction de l'opérateur qui se déduit naturellement de $N_{\theta, \rho}$ et qui envoie $\left.\rho\right|^{(a-b) / 2} \times \cdots \times \rho \|^{-(a+b) / 2+1}$ $\operatorname{sur} \rho||^{(a+b) / 2-1} \times \cdots \times\left.\rho\right|^{-(a-b) / 2}$. Par récurrence on sait que l'action de $\theta$ sur $S p(b-2, S t(a, \rho))$ s'obtient en restreignant celle que l'on définit naturellement sur

$$
S p(b-2, \rho)||^{(a-1) / 2} \times S p(b-2, S t(a-2, \rho)) \times S p(b-2, \rho)||^{-(a-1) / 2} .
$$

On représente $S p(b-2, \rho)$ comme sous-module de $\sigma_{S p, b-2, \rho}$ et comme ci-dessus on montre que l'action de $\theta$ sur (5) s'obtient en restreignant l'action de $\theta$ sur l'induite :

$$
\rho||^{(a-b) / 2+1} \times \cdots \times \rho||^{(a+b) / 2-2} \times S p(b-2, S t(a-2, \rho)) \times \rho||^{-(a+b) / 2+2} \times \cdots \times\left.\rho\right|^{-(a-b) / 2-1} .
$$

En remettant avec (4), on vient de montrer que l'action $\theta_{S t}$ sur $S p(b, S t(a, \rho))$ est celle que l'on obtient en restreignant l'action naturelle de $\theta$ sur l'induite :

$$
\begin{gathered}
\left.\rho\left\|\left.\right|^{(a-b) / 2} \times \cdots \times \rho||^{-(a+b) / 2+1} \times \rho\right\|\right|^{(a-b) / 2+1} \times \cdots \times \rho \|^{(a+b) / 2-2} \\
\times S p(b-2, S t(a-2, \rho)) \\
\times \rho||^{-(a+b) / 2+2} \times \cdots \times \rho||^{-(a-b) / 2-1} \times\left.\rho\right|^{(a+b) / 2-1} \times \cdots \times\left.\rho\right|^{-(a-b) / 2} .
\end{gathered}
$$

On fait les mêmes manipulations en partant de (2) pour trouver que l'action $\theta_{S p}$ est la restriction de l'action naturelle de $\theta$ sur l'induite :

$$
\begin{gathered}
\rho\left\|\left.\right|^{(a-b) / 2} \times \cdots \times \rho\right\|^{(a+b) / 2-1} \times\left.\rho\left\|\left.\right|^{(a-b) / 2-1} \times \cdots \times \rho\right\|\right|^{-(a+b) / 2+2} \\
\times S p(b-2, S t(a-2, \rho)) \\
\times \rho\left\|\left.\right|^{(a+b) / 2-2} \times \cdots \times\left.\rho\right|^{-(a-b) / 2+1} \times \rho\right\|^{-(a+b) / 2+1} \times \cdots \times \rho \|^{-(a-b) / 2} .
\end{gathered}
$$

Les induites (6) et (7) sont isomorphes (on le vérifiera) et il faut donc montrer que l'isomorphisme entrelace l'action de $\theta$, ainsi les restrictions seront les mêmes sur leur unique sous-module $S p(b, S t(a, \rho))$.

Construisons un isomorphisme de (6) sur (7); d'abord on remarque que l'induite

$$
\rho||^{(a-b) / 2} \times \rho\left\|^{(a-b) / 2-1} \cdots \times \rho\right\|^{-(a+b) / 2+1} \times \rho\left\|^{(a-b) / 2+1} \times \cdots \times \rho\right\|^{(a+b) / 2-2}
$$

est isomorphe à

$$
\rho||^{(a-b) / 2} \times \rho||^{(a-b) / 2+1} \times \cdots \times \rho||^{(a+b) / 2-2} \times \rho||^{(a-b) / 2-1} \times \cdots \times \rho||^{-(a+b) / 2+1},
$$

où on utilise simplement le fait que pour $y \in[(a-b) / 2-1,-(a+b) / 2+1]$ et $y^{\prime} \in[(a-b) / 2+1,(a+$ $b) / 2-2], y-y^{\prime}<-1$. On peut construire un isomorphisme à l'aide des opérateurs d'entrelacement standard par exemple et en transportant cet isomorphisme par $\theta$, on construit un isomorphisme de (6) sur

$$
\begin{aligned}
& \rho||^{(a-b) / 2} \times \rho||^{(a-b) / 2+1} \times \cdots \times \rho||^{(a+b) / 2-2} \times \rho||^{(a-b) / 2-1} \times \cdots \times \rho||^{-(a+b) / 2+1} \times S p(b-2, S t(a-2, \rho)) \\
& \times \rho||^{(a+b) / 2-1} \times \cdots \times\left.\rho\right|^{-(a-b) / 2+1} \times\left.\rho\right|^{-(a+b) / 2+2} \times \cdots \times\left.\rho\right|^{-(a-b) / 2-1)} \times \rho||^{-(a-b) / 2} .
\end{aligned}
$$

Cet isomorphisme est compatible aux actions de $\theta$ définies essentiellement car il n'y a qu'une action de $\theta$ naturelle sur ces induites une fois que l'on a fixé l'action sur $S p(b-2, S t(a-2, \rho))$, mais on renvoie à 1.9 pour une démonstration précise. De même on entrelace l'action de $\theta$ sur (7) avec l'action de $\theta$ sur l'induite (9) ci-dessous qui lui est isomorphe :

$$
\begin{gathered}
\left.\rho\left\|\left.\right|^{(a-b) / 2} \times \rho\right\|\right|^{(a-b) / 2+1} \times \cdots \times \rho\left\|\left.\right|^{(a+b) / 2-2} \times \rho\right\|^{(a-b) / 2-1} \times \cdots \times \rho\left\|^{-(a+b) / 2+2} \times \rho\right\| \|^{(a+b) / 2-1} \\
\times S p(b-2, S t(a-2, \rho)) \times
\end{gathered}
$$




$$
\rho||^{-(a+b) / 2+1} \times \rho||^{(a+b) / 2-2} \times \cdots \times \rho||^{-(a-b) / 2+1} \times \rho||^{-(a+b) / 2+2} \times \cdots \times\left.\rho\right|^{-(a-b) / 2-1} \times \rho||^{-(a-b) / 2} .
$$

Ici on a fait commuter $\left.\rho\right|^{(a+b) / 2-1}$ avec les induites $\left.\rho\right|^{y}$ pour $y \in[(a-b) / 2-1,-(a+b) / 2+2]$ et $\rho||^{-(a+b) / 2+1}$ avec les induites $\rho||^{-y}$ pour les mêmes valeurs de $y$. Il reste à construire un isomorphisme :

$\rho||^{(a+b) / 2-1} \times S p(b-2, S t(a-2, \rho)) \times \rho||^{-(a+b) / 2+1} \simeq \rho||^{-(a+b) / 2+1} \times S p(b-2, S t(a-2, \rho)) \times \rho||^{(a+b) / 2-1}$

entrelaçant les actions de $\theta$. On glisse un paramètre $s \in \mathbb{C}$ et on regarde l'entrelacement :

$$
\begin{gathered}
\rho||^{(a+b) / 2-1+s} \times S p(b-2, S t(a-2, \rho)) \times\left.\rho\right|^{-(a+b) / 2+1-s} \rightarrow \\
\rho||^{-(a+b) / 2+1-s} \times S p(b-2, S t(a-2, \rho)) \times\left.\rho\right|^{(a+b) / 2-1+s} .
\end{gathered}
$$

En $s=0$ les induites écrites sont irréductibles car le support cuspidal de $S p(b-2, S t(a-2, \rho))$ est formé de représentation de la forme $\left.\rho\right|^{x}$ avec $x \in[-(a+b) / 2+3,(a+b) / 2-3]$. Pour obtenir l'assertion cherchée on va démontrer plus généralement le lemme ci-dessous (1.9).

\subsection{Opérateurs d'entrelacement et action de $\theta$}

Fixons d'abord quelques notations : soit $\pi_{i}$ pour $i=1,2$ des représentations de groupes linéaires et soit $s_{i} \in \mathbb{C}$ pour $i=1,2$. On note $M\left(\pi_{1}, \pi_{2}, s\right)$ l'opérateur d'entrelacement standard de l'induite

$$
M\left(\pi_{1}, \pi_{2}, s\right): \quad \pi_{1}||^{s_{1}} \times \pi_{2}||^{s_{2}} \rightarrow \pi_{2}||^{s_{2}} \times \pi_{1}||^{s_{1}} .
$$

On définit de même

$$
M\left({ }^{\theta} \pi_{1},{ }^{\theta} \pi_{2}, s\right): \quad{ }^{\theta} \pi_{2}||^{-s_{2}} \times{ }^{\theta} \pi_{1}||^{-s_{1}} \rightarrow{ }^{\theta} \pi_{1}||^{-s_{1}} \times{ }^{\theta} \pi_{2}||^{-s_{2}} .
$$

Ces opérateurs ne dépendent que de $s=s_{1}-s_{2}$ dans les 2 cas. On identifie les espaces de $V_{\pi_{i}}$ et $V_{\pi_{i}}$ pour $i=1,2$. On note $i$ l'isomorphisme de $V_{\pi_{2}} \otimes V_{\pi_{1}}$ sur $V_{\pi_{1}} \otimes V_{\pi_{2}}$ qui échange les facteurs . On remarque que l'application qui à $\phi \in \pi_{2}||^{s_{2}} \times \pi_{1}||^{s_{1}}$ associe $i \circ \theta \phi$ est à valeurs dans ${ }^{\theta} \pi_{1}||^{-s_{1}} \times{ }^{\theta} \pi_{2}||^{-s_{2}}$ et on note $i \circ \Theta$ cet opérateur. On définit $i_{\theta}$ l'isomorphisme inverse de $i$ et on a aussi l'opérateur

$$
i_{\theta} \circ \Theta: \pi_{1}||^{s_{1}} \times \pi_{2}||^{s_{2}} \rightarrow^{\theta} \pi_{2}||^{-s_{2}} \times{ }^{\theta} \pi_{1}||^{-s_{1}} .
$$

Lemme : on a l'égalité des opérateurs : $i \circ \Theta \circ M\left(\pi_{1}, \pi_{2}, s\right)=M\left({ }^{\theta} \pi_{2},{ }^{\theta} \pi_{1}, s\right) \circ i_{\theta} \circ \Theta$. en tant qu'opérateurs de $\pi_{1}||^{s_{1}} \times \pi_{2}||^{s_{2}}$ dans ${ }^{\theta} \pi_{1}||^{-s_{1}} \times{ }^{\theta} \pi_{2}||^{-s_{2}}$.

On calcule pour $s$ de partie réelle grande en utilisant la réalisation des opérateurs d'entrelacement comme intégrales et l'assertion est immédiate.

\subsection{Fin de la preuve de 1.8}

Revenons à (10); l'action de $\theta$ sur l'induite de gauche est, avec une généralisation des notations, $i \circ \Theta$ et sur l'induite de droite $i_{\theta} \circ \Theta$. L'entrelacement cherché résulte aisément du lemme. Cela termine la preuve de la proposition 1.8

\subsection{Définition de $\operatorname{Jord}(\psi), \operatorname{Jord}_{\rho}(\psi)$ et convention d'écriture}

On décompose $\psi$ en somme de représentations irréductibles de $W_{F} \times S L(2, \mathbb{C}) \times S L(2, \mathbb{C})$; pour $c$ un entier on note, ici et ici seulement, $[c]$ la représentation de $S L(2, \mathbb{C})$ irréductible de dimension $c$ une sous-représentation irréductible incluse dans $\psi$ est un produit tensoriel, $\rho \otimes[a] \otimes[b]$ où $\rho$ s'identifie par la correspondance de Langlands à une représentation irréductible de $W_{F}$ et $a, b$ sont des entiers. Cette décomposition en sous-représentations irréductibles donne donc un ensemble, avec multiplicité, de triplets $\{(\rho, a, b)\}$ tel que

$$
\oplus_{(\rho, a, b)} \rho \otimes[a] \otimes[b]=\psi .
$$

On transforme cet ensemble en un ensemble de quadruplets $(\rho, A, B, \zeta)$ en posant pour chaque $\rho, a, b$ comme ci-dessus, $A=(a+b) / 2-1, B=|a-b| / 2$ et $\zeta$ le signe de $a-b$ ou indifféremment \pm 1 si 
$a=b$. La restriction de la représentation $\rho \otimes[a] \otimes[b]$ de $W_{F} \times S L(2, \mathbb{C}) \times S L(2, \mathbb{C})$ à la diagonale est la somme des représentations $\rho \otimes[c]$ où $c$ parcourt l'intervalle $[2 A+1,2 B+1]_{2}$, où le 2 en indice indique que l'on ne prend que les entiers ayant même parité que les bornes; d'où la signification de $A$ et $B$. On pose alors $\pi(\rho, A, B, \zeta):=S p(b, S t(a, \rho))$. On note l'ensemble des quadruplets qui décrivent la représentation définie par $\psi$, Jord $(\psi)$; pour $\rho$ fixé, on note $\operatorname{Jord}_{\rho}(\psi)$ l'ensemble des quadruplets $\left(\rho^{\prime}, A^{\prime}, B^{\prime}, \zeta^{\prime}\right)$ de $\operatorname{Jord}(\psi)$ vérifiant $\rho \simeq \rho^{\prime}$.

\section{Attention à la convention commode sur $\operatorname{Jord}_{\rho}(\psi)$.}

Cet ensemble, $\operatorname{Jord}_{\rho}(\psi)$ est encore la réunion de 2 ensembles qui n'ont rien à voir entre eux, $\operatorname{Jord}_{\rho, \text { ent }}(\psi)$ et $\operatorname{Jord}_{\rho, 1 / 2 \text { ent }}(\psi)$ où $(\rho, A, B, \zeta) \in \operatorname{Jord}_{\rho, \text { ent }}(\psi)$ précisément quand $A, B$ sont des entiers. Dans la suite, quand on aura un quadruplet $(\rho, A, B, \zeta) \in \operatorname{Jord}(\psi)$ fixé on abrégera en $\operatorname{Jord}_{\rho}(\psi)$ ce qui devrait être "ent" ou "1/2ent" ceci étant fixé par $A, B$.

\subsection{Définition de $\pi(\psi)$ pour $\psi$ de restriction discrète à la diagonale}

On réutilise la notation $\Delta$ de l'introduction : $\Delta$ est le plongement diagonal de $S L(2, \mathbb{C})$ dans $S L(2, \mathbb{C}) \times S L(2, \mathbb{C})$ que l'on prolonge par l'identité sur $W_{F}$ en un morphisme de $W_{F} \times S L(2, \mathbb{C})$ dans $W_{F} \times S L(2, \mathbb{C}) \times S L(2, \mathbb{C})$. On suppose ici que la restriction de $\psi \circ \Delta$ à $W_{F} \times S L(2, \mathbb{C})$ définit une représentation sans multiplicités. L'hypothèse faite sur la restriction de $\psi$ à la diagonale se traduit par le fait que si $(\rho, A, B, \zeta)$ et $\left(\rho^{\prime}, A^{\prime}, B^{\prime}, \zeta^{\prime}\right)$ sont dans $\operatorname{Jord}(\psi)$ alors soit $\rho \neq \rho^{\prime}$ soit $[B, A] \cap\left[B^{\prime}, A^{\prime}\right]=\emptyset$; cela entraîne que la représentation $<\rho||^{\zeta B}, \cdots,\left.\rho\right|^{-\zeta A}>\times \pi\left(\rho^{\prime}, A^{\prime}, B^{\prime}, \zeta^{\prime}\right)$ est irréductible et qu'il en est de même de la représentation $\pi\left(\rho^{\prime}, A^{\prime}, B^{\prime}, \zeta^{\prime}\right) \times<\left.\rho\right|^{-\zeta A}, \cdots,\left.\rho\right|^{\zeta B}>$. Dans tout ce qui suit, quand on a fixé $(\rho, A, B, \zeta) \in \operatorname{Jord}(\psi)$ on note $\psi^{\prime}$ le morphisme qui se déduit de $\psi$ en "enlevant" la représentation de $W_{F} \times S L(2, \mathbb{C}), \times S L(2, \mathbb{C})$ correspondant à $(\rho, A, B, \zeta) ; \psi^{\prime}$ est donc à valeurs dans $G L\left(N-d_{\rho} a b, \mathbb{C}\right)$ (où $(\rho, a, b)$ correspond à $\left.(\rho, A, B, \zeta)\right)$. Une écriture $\left(\psi^{\prime},\left(\rho, A^{\prime}, B^{\prime}, \zeta^{\prime}\right)\right)$ représente le morphisme $\psi^{\prime \prime}$ tel que $\left.\operatorname{Jord}\left(\psi^{\prime \prime}\right)=\operatorname{Jord}\left(\psi^{\prime}\right) \cup\left(\rho, A^{\prime}, B^{\prime}, \zeta\right)\right)$.

La définition de l'action de $\theta$ se fait en plusieurs temps, c'est un point vraiment délicat. D'abord on suppose que pour tout $(\rho, A, B, \zeta) \in \operatorname{Jord}(\psi), A=B=0$; dans ce cas $\pi(\psi)$ est une induite de cuspidales $\theta$-invariantes toutes distinctes; avec les opérateurs d'entrelacement, on définit une action de $\theta$. On la normalise à la Whittaker comme expliqué dans l'introduction.

Ensuite on fait les constructions par récurrence à partir de ce choix. Supposons pour le moment, en plus, que pour tout $\left(\rho, A^{\prime}, B^{\prime}, \zeta^{\prime}\right) \in \operatorname{Jord}_{\rho}(\psi), A^{\prime}=B^{\prime}$; dans les notations initiales, c'est la condition inf $(a, b)=1$. Ici on note $\left(\rho, A_{0}, B_{0}, \zeta_{0}\right)$ l'élément de $\operatorname{Jord}_{\rho}(\psi)$ tel que $B_{0}$ soit minimum. Il y a encore 2 cas à distinguer.

Le cas où $B_{0} \neq 0$; en tant que représentation de $G L(N)$, on vérifie que $\pi(\psi)$ est l'unique sousmodule irréductible de l'induite :

$$
\tau:=\rho||^{\zeta B_{0}} \times \pi\left(\psi^{\prime},\left(\rho, B_{0}-1, B_{0}-1, \zeta\right)\right) \times \pi\left(\psi^{\prime}\right) \times \rho||^{-\zeta B_{0}}
$$

$\left(\rho, B_{0}-1, B_{0}-1, \zeta\right)$ n'intervient pas si $B_{0}=1 / 2$; en effet que $\pi(\psi)$ soit un sous-module de $\tau$ résulte immédiatement des définitions et le point est de vérifier que l'induite de droite a un unique sousmodule irréductible. On vérifie cela par réciprocité de Frobenius. On utilise la notation $J a c_{x}^{\theta}$ de 1.4 relative à notre $\rho$ fixé. Et il suffit de vérifier que

$$
J a c_{\zeta B_{0}}^{\theta} \tau=\pi\left(\psi^{\prime},\left(\rho, B_{0}-1, B_{0}-1, \zeta\right)\right) .
$$

Or on a $J a c_{x} \pi\left(\psi^{\prime}\right) \neq 0$ seulement si $x$ est de la forme $\zeta^{\prime} B^{\prime}$ avec $\left(\rho, A^{\prime}, B^{\prime}, \zeta^{\prime}\right) \in J o r d\left(\psi^{\prime}\right)$; or pour un tel $\zeta^{\prime} B^{\prime}$, on a $\zeta^{\prime} B^{\prime} \notin\left[\zeta_{0} B_{0},-\zeta_{0} B_{0}\right]$ par l'hypothèse sur la restriction de $\psi$ à la diagonale. Donc $J a c_{\zeta B_{0}} \pi\left(\psi^{\prime},\left(\rho, B_{0}-1, B_{0}-1, \zeta\right)\right)=0$. On a une assertion analogue pour calculer $J a c_{-\zeta B_{0}}$ "à droite", c'est à dire que l'on projette le module de Jacquet sur l'espace des représentations de la forme $\sigma^{\prime} \otimes$ $\rho||^{-\zeta B_{0}}$. Et les formules standard montrent alors l'assertion cherchée.

D'où, en écrivant les définitions :

$$
\left.\pi(\psi) \hookrightarrow \rho\right|^{\zeta B_{0}} \times \pi\left(\rho, B_{0}-1, B_{0}-1\right) \times \pi\left(\psi^{\prime}\right) \times\left.\rho\right|^{-\zeta B_{0}}
$$


L'induite écrite a une action de $\theta$ dès que $\pi\left(\rho, B_{0}-1, B_{0}-1\right) \times \pi\left(\psi^{\prime}\right)$ en a une et l'action sur l'induite ne dépend que de l'action sur $\pi\left(\rho, B_{0}-1, B_{0}-1\right) \times \pi\left(\psi^{\prime}\right)$. Par unicité du sous-module, on en déduit une action sur $\pi(\psi)$; c'est ainsi que l'on met une action sur $\pi(\psi)$ par induction.

On suppose maintenant que $B_{0}=0$ et qu'il existe $\left(\rho, B_{1}, B_{1}, \zeta_{1}\right) \in \operatorname{Jord}(\psi)$ avec $B_{1} \neq 0$. On fixe $B_{1}$ minimum avec cette propriété et on note $\psi_{1}$ le morphisme qui se déduit de $\psi$ en enlevant les 2 blocs, celui relatif à $B_{0}$ et celui relatif à $B_{1}$. Montrons d'abord l'inclusion (cf 1.5 pour la notation) :

$$
\pi(\psi) \hookrightarrow<\rho||^{\zeta_{1} B_{1}}, \cdots, \rho>\times \pi\left(\psi_{1}\right) \times<\rho, \cdots,\left.\rho\right|^{-\zeta_{1} B_{1}}>
$$

Or par minimalité de $B_{1}$, l'entrelacement :

$$
\pi\left(\psi_{1}\right) \times<\rho, \cdots, \rho||^{-\zeta_{1} B_{1}}>\rightarrow<\rho, \cdots, \rho||^{-\zeta_{1} B_{1}}>\times \pi\left(\psi_{1}\right)
$$

est un isomorphisme. Comme

$$
\pi(\psi) \simeq \rho \times<\rho||^{\zeta_{1} B_{1}}, \cdots,\left.\rho\right|^{-\zeta_{1} B_{1}}>\times \pi\left(\psi_{1}\right)
$$

le seul point à vérifier est que

$$
\rho \times<\rho||^{\zeta_{1} B_{1}}, \cdots, \rho||^{-\zeta_{1} B_{1}}>\hookrightarrow<\rho||^{\zeta_{1} B_{1}}, \cdots, \rho>\times<\rho, \cdots, \rho||^{-\zeta_{1} B_{1}}>
$$

ce qui est un cas particulier des résultats de Zelevinsky.

L'induite $\left(^{*}\right)$ contient même $\pi(\psi)$ avec multiplicité 1 en tant que sous-quotient par un calcul élémentaire de module de Jacquet (ici on utilise le fait que $\psi \circ \Delta$ est sans multiplicités). Ainsi toute action de $\theta$ sur $\pi\left(\psi_{1}\right)$ donne une action de $\theta$ sur l'induite et par restriction une action de $\theta$ sur $\pi(\psi)$. Cela termine les définitions dans le cas élémentaire.

Pour la suite, on a besoin de remarquer que

$$
<\rho, \cdots, \rho||^{-\zeta_{1} B_{1}}>\times \pi\left(\psi_{1}\right) \times<\rho||^{\zeta_{1} B_{1}}, \cdots, \rho>.
$$

admet $\pi(\psi)$ comme unique quotient irréductible : ce résultat est dual du précédent et résulte donc du fait que $\pi(\psi)$ est autoduale. Toute action de $\theta$ sur $\pi\left(\psi_{1}\right)$ se prolonge donc canoniquement à $(* *)$ et par restriction donne une action de $\theta$ sur $\pi(\psi)$. On montre que les 2 actions ainsi définies coïncident : pour cela on construit un entrelacement pour $s \in \mathbb{C}$ voisin de 0 :

$$
<\rho, \cdots,\left.\rho\right|^{-\zeta_{1} B_{1}}>||^{s} \times \pi\left(\psi_{1}\right) \times<\rho||^{\zeta_{1} B_{1}}, \cdots, \rho>||^{-s}
$$

dans

$$
<\rho||^{\zeta_{1} B_{1}}, \cdots, \rho>||^{-s} \times \pi\left(\psi_{1}\right) \times<\rho, \cdots,\left.\rho\right|^{-\zeta_{1} B_{1}}>||^{s} .
$$

D'après 1.9, les opérateurs d'entrelacement standard permettent de construire un tel entrelacement compatible aux prolongements canonique de l'action de $\theta$ sur $\pi\left(\psi_{1}\right)$; en $s=0$, l'entrelacement n'a pas de raison d'être holormorphe non nul mais on peut toujours obtenir cela à condition de multiplier par $s^{z}$ avec $z \in \mathbb{Z}$ convenable. On peut alors évaluer en $s=0$; l'image est nécessairement égale à $\pi(\psi)$ et on obtient l'entrelacement des actions de $\theta$ comme annoncé.

Supposons maintenant que $\operatorname{Jord}(\psi)$ contient un quadruplet $(\rho, A, B, \zeta)$ avec $A>B$. Ici on vérifie que $\pi(\psi)$, comme représentation de $G L(N, F)$, est l'unique sous-module irréductible de l'induite :

$$
\sigma:=<\rho||^{\zeta B}, \cdots, \rho||^{-\zeta A}>\times \pi(\rho, A-1, B+1, \zeta) \times<\rho||^{\zeta A}, \cdots, \rho||^{-\zeta B}>\times \pi\left(\psi^{\prime}\right),
$$

où $\pi(\rho, A-1, B+1, \zeta)$ est la représentation triviale de $G L(0, F)$ si $A=B+1$. L'assertion est un peu plus difficile que ci-dessus. En effet, il faut d'abord montrer avec les notations de 1.4 que

$$
J a c_{\zeta B, \cdots,-\zeta A} \sigma=\pi(\rho, A-1, B+1, \zeta) \times<\left.\rho\right|^{\zeta A}, \cdots, \rho \|^{-\zeta B}>\times \pi\left(\psi^{\prime}\right) .
$$


Pour cela, on vérifie que si ce n'est pas vrai il existe un sous-segment $[x,-\zeta A]$ de $[\zeta B,-\zeta A]$ tel que

$$
J a c_{x, \cdots,-\zeta A}\left(\pi(\rho, A-1, B+1, \zeta) \times<\rho||^{\zeta A}, \cdots,\left.\rho\right|^{-\zeta B}>\times \pi\left(\psi^{\prime}\right)\right) \neq 0 .
$$

Or $\rho||^{-\zeta A}$ ne se trouve pas dans le support cuspidal de $\pi(\rho, A-1, B+1, \zeta) \times<\rho||^{\zeta A}, \cdots,\left.\rho\right|^{-\zeta B}>$, et il faudrait donc encore qu'il existe un sous-segment $\left[x^{\prime},-\zeta A\right]$ de $[\zeta B,-\zeta A]$ tel que $J a c_{x^{\prime}, \cdots,-\zeta A} \pi\left(\psi^{\prime}\right) \neq 0$. On récrit $\pi\left(\psi^{\prime}\right)$ comme une induite de représentations de la forme $\pi\left(\rho^{\prime}, A^{\prime}, B^{\prime}, \zeta^{\prime}\right)$. Et quitte à reduire encore $\left[x^{\prime},-\zeta A\right]$ il devrait exister $\left(\rho^{\prime}, A^{\prime}, B^{\prime}, \zeta^{\prime}\right) \in \operatorname{Jord}\left(\psi^{\prime}\right)$ tel que $J a c_{x^{\prime}, \cdots,-\zeta A} \pi\left(\rho^{\prime}, A^{\prime}, B^{\prime}, \zeta^{\prime}\right) \neq 0$; cela entraîne $\rho \simeq \rho^{\prime}$ et $x^{\prime}=\zeta^{\prime} B^{\prime}$, en particulier $B^{\prime} \leq A$; par l'hypothèse de restriction discrète à la diagonale, cela assure que $A^{\prime}$ vérifie $A^{\prime}<B$ et $\rho \|^{-\zeta A}$ n'est pas dans le support cuspidal de $\pi\left(\rho, A^{\prime}, B^{\prime}, \zeta^{\prime}\right)$ ce qui donne la contradiction. La démonstration n'est pas finie; on utilise le fait que dans (1) et dans (2) on peut mettre $<\rho||^{\zeta A}, \cdots,\left.\rho\right|^{-\zeta B}>$ en dernière position et par exemple en conjuguant par $\theta$, il suffit de démontrer que l'induite

$$
J a c_{\zeta B, \cdots,-\zeta A}<\rho||^{\zeta B}, \cdots, \rho||^{-\zeta A}>\times \pi(\rho, A-1, B+1, \zeta) \times \pi\left(\psi^{\prime}\right)=\pi(\rho, A-1, B+1, \zeta) \times \pi\left(\psi^{\prime}\right) .
$$

C'est clairement la même démonstration qui prouve l'assertion.

On a l'isomorphisme

$$
<\rho||^{\zeta A}, \cdots,\left.\rho\right|^{-\zeta B}>\times \pi\left(\psi^{\prime}\right) \simeq \pi\left(\psi^{\prime}\right) \times<\rho||^{\zeta A}, \cdots,\left.\rho\right|^{-\zeta B}>,
$$

un tel isomorphisme se construit avec des opérateurs d'entrelacement standard rendus holomorphes en 0 . En effet on plonge $\pi\left(\psi^{\prime}\right)$ dans une induite de la forme

$$
\times_{\left(\rho^{\prime}, A^{\prime}, B^{\prime}, \zeta^{\prime}\right) \in \operatorname{Jord}\left(\psi^{\prime}\right)} \times_{k \in\left[0, A^{\prime}-B^{\prime}\right]}<\rho^{\prime}||^{\zeta^{\prime}\left(B^{\prime}+k\right)}, \cdots, \rho||^{-\zeta^{\prime}\left(A^{\prime}-k\right)}>
$$

et il suffit de démontrer que pour tout $\left(\rho^{\prime}, A^{\prime}, B^{\prime}, \zeta^{\prime}\right), k$ comme ci-dessus :

$$
\begin{aligned}
& <\rho||^{\zeta A}, \cdots, \rho||^{-\zeta B}>\times<\rho^{\prime}||^{\zeta^{\prime}\left(B^{\prime}+k\right)}, \cdots, \rho||^{-\zeta^{\prime}\left(A^{\prime}-k\right)}>\simeq \\
& <\rho^{\prime}||^{\zeta^{\prime}\left(B^{\prime}+k\right)}, \cdots,\left.\rho\right|^{-\zeta^{\prime}\left(A^{\prime}-k\right)}>\times<\rho||^{\zeta A}, \cdots,\left.\rho\right|^{-\zeta B}>.
\end{aligned}
$$

L'hypothèse que $\psi$ est de restriction discrète à la diagonale assure que soit $A^{\prime}<B$ soit $A<B^{\prime}$ d'où aussi soit $A^{\prime}-k<B$ soit $A<B^{\prime}+k$. Ainsi le segment $[\zeta A,-\zeta B]$ soit contient le segment $\left[\zeta^{\prime}\left(B^{\prime}+k\right),-\zeta^{\prime}\left(A^{\prime}-k\right)\right]$ soit est inclus dans ce segment même si les propriétés de croissance des segments ne sont pas les mêmes. On construit alors l'isomorphisme comme restriction d'un isomorphisme où on garde la représentation correspondant au segment le plus long et on remplace l'autre par l'induite évidente ; par exemple supposons que $A<B^{\prime}$, pour tout $x \in[\zeta A,-\zeta B], x \in\left[\zeta^{\prime}\left(B^{\prime}+k\right),-\zeta^{\prime}\left(A^{\prime}-k\right)\right]$ et l'induite $\rho||^{x} \times<\rho^{\prime}||^{\zeta^{\prime}\left(B^{\prime}+k\right)}, \cdots, \rho||^{-\zeta^{\prime}\left(A^{\prime}-k\right)}>$ est irréductibile d'après les résultats de Zelevinsky. D'où

$$
\times_{x \in[\zeta A,-\zeta B]} \rho||^{x} \times<\rho^{\prime}||^{\zeta^{\prime}\left(B^{\prime}+k\right)}, \cdots, \rho||^{-\zeta^{\prime}\left(A^{\prime}-k\right)}>\simeq<\rho^{\prime}||^{\zeta^{\prime}\left(B^{\prime}+k\right)}, \cdots, \rho||^{-\zeta^{\prime}\left(A^{\prime}-k\right)}>\times_{x \in[\zeta A,-\zeta B]} \rho||^{x},
$$

isomorphisme que l'on peut construire avec les opérateurs d'entrelacements standard rendu holomorphes en $s=0$ (on a remplacé $\left.<\rho^{\prime}||^{\prime}\left(B^{\prime}+k\right), \cdots, \rho||^{-\zeta^{\prime}\left(A^{\prime}-k\right)}>\operatorname{par}<\rho^{\prime}||^{\prime}\left(B^{\prime}+k\right), \cdots, \rho||^{-\zeta^{\prime}\left(A^{\prime}-k\right)}\right\rangle$ ||$\left.^{s}\right)$. D'où l'assertion.

On note $\psi_{-}$le morphisme qui se déduit de $\psi$ en remplçant $(\rho, A, B, \zeta)$ par $(\rho, A-1, B+1, \zeta)$ et on obtient donc que $\pi(\psi)$ est l'unique sous-module irréductible de l'induite :

$$
<\rho||^{\zeta B}, \cdots,\left.\rho\right|^{-\zeta A}>\times \pi\left(\psi_{-}\right) \times<\left.\rho\right|^{\zeta A}, \cdots,\left.\rho\right|^{-\zeta B}>.
$$

On remarque que le morphisme, $\psi_{-}$a la même propriété de restriction discrète à la diagonale que $\psi$. Ainsi on peut munir par induction l'induite que l'on vient d'écrire d'une action de $\theta$ qui ne dépend que de l'action de $\theta$ sur $\pi\left(\psi_{-}\right)$. Par unicité du sous-module, cela donne une action de $\theta$ sur $\pi(\psi)$. 
Lemme. L'action que l'on vient de mettre sur $\pi(\psi)$ est indépendante du choix de $(\rho, A, B, \zeta)$ tel que $A>B$.

On fixe $(\rho, A, B, \zeta)$ et $\left(\rho^{\prime}, A^{\prime}, B^{\prime}, \zeta^{\prime}\right)$ dans $\operatorname{Jor} d(\psi)$ tel que $A>B$ et $A^{\prime}>B^{\prime}$. On note $\psi^{\prime \prime}$ le morphisme qui se déduit de $\psi$ en enlevant ces 2 blocs. On représente $\pi(\psi)$ comme sous-module irréductible de l'induite :

$$
\begin{aligned}
<\rho||^{\zeta B}, \cdots, \rho||^{-\zeta A}> & \times<\rho^{\prime}||^{\zeta^{\prime} B^{\prime}}, \cdots, \rho^{\prime}||^{-\zeta^{\prime} A^{\prime}}>\times \pi\left(\psi^{\prime \prime},(\rho, A-1, B+1, \zeta),\left(\rho^{\prime}, A^{\prime}-1, B^{\prime}+1, \zeta^{\prime}\right)\right) \\
& \times<\rho^{\prime}||^{\zeta^{\prime} A^{\prime}}, \cdots, \rho^{\prime}||^{-\zeta^{\prime} B^{\prime}}>\times<\rho||^{\zeta B}, \cdots,\left.\rho\right|^{-\zeta A}>
\end{aligned}
$$

et comme sous-module de l'induite :

$$
\begin{aligned}
<\rho^{\prime}||^{\zeta^{\prime} B^{\prime}}, \cdots, \rho^{\prime}||^{-\zeta^{\prime} A^{\prime}}> & \times<\rho||^{\zeta B}, \cdots, \rho||^{-\zeta A}>\times \pi\left(\psi^{\prime \prime},(\rho, A-1, B+1, \zeta),\left(\rho^{\prime}, A^{\prime}-1, B^{\prime}+1, \zeta^{\prime}\right)\right) \\
& <\rho||^{\zeta B}, \cdots, \rho||^{-\zeta A}>\times<\rho^{\prime}||^{\zeta^{\prime} A^{\prime}}, \cdots, \rho^{\prime}||^{-\zeta^{\prime} B^{\prime}}>
\end{aligned}
$$

Et le point est de montrer que les prolongements canoniques de l'action de $\theta$ sur $\pi\left(\psi^{\prime \prime},(\rho, A-1, B+\right.$ $\left.1, \zeta),\left(\rho^{\prime}, A^{\prime}-1, B^{\prime}+1, \zeta^{\prime}\right)\right)$ à $(3)$ et à $(4)$ ont la même restriction à $\pi(\psi)$. On remplace par exemple dans $(3),<\rho||^{\zeta B}, \cdots,\left.\rho\right|^{-\zeta A}>\operatorname{par}<\rho||^{\zeta B}, \cdots, \rho||^{-\zeta A}>\|\left.\right|^{S}$ et $<\rho||^{\zeta B}, \cdots,\left.\rho\right|^{-\zeta A}>$ en $<\rho||^{\zeta B}, \cdots,\left.\rho\right|^{-\zeta A}>\|\left.\right|^{-s}$. On peut alors utiliser l'opérateur d'entrelacement standard

$$
\begin{aligned}
& <\rho||^{\zeta B}, \cdots, \rho||^{-\zeta A}>||^{s} \times<\rho^{\prime}||^{\zeta^{\prime} B^{\prime}}, \cdots, \rho^{\prime}||^{-\zeta^{\prime} A^{\prime}}>\rightarrow \\
& <\rho^{\prime}||^{\zeta^{\prime} B^{\prime}}, \cdots, \rho^{\prime}||^{-\zeta^{\prime} A^{\prime}}>\times<\rho||^{\zeta B}, \cdots,\left.\rho\left\|\left.\right|^{-\zeta A}>\right\|\right|^{s}
\end{aligned}
$$

Composé avec son analogue pour échanger les 2 derniers facteurs. On vient de vérifier ci-dessus, qu'en le normalisant correctement, il devient un isomorphisme en $s=0$ (la normalisation consiste simplement à le rendre holomorphe en $s=0$ ). Il est clair comme en 1.9 que l'on a ainsi construit un entrelacement entre le prolongement canonique de l'action de $\theta$ sur $\pi\left(\psi^{\prime \prime},(\rho, A-1, B+1, \zeta),\left(\rho^{\prime}, A^{\prime}-1, B^{\prime}+1, \zeta^{\prime}\right)\right)$ à l'induite :

$$
\begin{aligned}
<\rho||^{\zeta B}, \cdots, \rho||^{-\zeta A}> & ||^{s} \times \times<\rho^{\prime}||^{\zeta^{\prime} B^{\prime}}, \cdots, \rho^{\prime}||^{-\zeta^{\prime} A^{\prime}}>\times \pi\left(\psi^{\prime \prime},(\rho, A-1, B+1, \zeta),\left(\rho^{\prime}, A^{\prime}-1, B^{\prime}+1, \zeta^{\prime}\right)\right) \\
& \times<\rho^{\prime}||^{\zeta^{\prime} A^{\prime}}, \cdots, \rho^{\prime}||^{-\zeta^{\prime} B^{\prime}}>\times<\rho||^{\zeta B}, \cdots,\left.\rho\right|^{-\zeta A}>\|\left.\right|^{-s}
\end{aligned}
$$

et le prolongement canonique de cette même action à l'induite :

$$
\begin{gathered}
<\rho^{\prime}||^{\zeta^{\prime} B^{\prime}}, \cdots, \rho^{\prime}||^{-\zeta^{\prime} A^{\prime}}>\times<\rho||^{\zeta B}, \cdots, \rho||^{-\zeta A}>||^{s} \times \pi\left(\psi^{\prime \prime},(\rho, A-1, B+1, \zeta),\left(\rho^{\prime}, A^{\prime}-1, B^{\prime}+1, \zeta^{\prime}\right)\right) \\
<\rho||^{\zeta B}, \cdots, \rho||^{-\zeta A}>\|\left.\right|^{-s} \times<\rho^{\prime}||^{\zeta^{\prime} A^{\prime}}, \cdots, \rho^{\prime}||^{-\zeta^{\prime} B^{\prime}}>
\end{gathered}
$$

En faisant $s=0$ on obtient le résultat cherché.

Lemme. L'action de $\theta$ mise sur $\pi(\psi)$ est indépendante du choix de $\rho$.

En effet fixons, $\rho, \rho^{\prime}$ tel que $\operatorname{Jord}_{\rho}(\psi) \neq \emptyset$ et $\operatorname{Jord}_{\rho^{\prime}}(\psi) \neq \emptyset$. On suppose que $\rho \not \rho^{\prime}$. Pour tout $x, y \in \mathbb{R}$, l'induite $\rho||^{x} \times \rho^{\prime}||^{y}$ est irréductible. Il suffit de faire commuter comme ci-dessus les définitions données en utilisant $\rho$ et celles données en utilisant $\rho^{\prime}$ pour obtenir l'asssertion.

\subsection{Quelques propriétés de l'action de $\theta$}

Dans ce paragraphe on suppose encore que la restriction de $\psi \circ \Delta$ est sans multiplicités vue comme représentation de $W_{F} \times S L(2, \mathbb{C})$. Soit $(\rho, A, B, \zeta) \in \operatorname{Jord}(\psi)$ avec $B \geq 1$; on a défini $\psi^{\prime}$ dans 1.12 . On suppose ici que pour tout $\left(\rho^{\prime}, A^{\prime}, B^{\prime}, \zeta^{\prime}\right) \in \operatorname{Jord}\left(\psi^{\prime}\right)$ avec $\rho^{\prime} \simeq \rho, A^{\prime} \neq B-1$. Le morphisme $\psi_{-}$ qui ici est défini en remplaçant $(\rho, A, B, \zeta) \operatorname{par}(\rho, A-1, B-1, \zeta)$ vérifie encore que $\psi_{-} \circ \Delta$ est sans multiplicités. 
Lemme. Sous l'hypothèse ci-dessus, l'induite $<\rho||^{\zeta B}, \cdots,\left.\rho\right|^{\zeta A}>\times \pi\left(\psi_{-}\right) \times<\rho||^{-\zeta A}, \cdots, \rho||^{-\zeta B}>$ a un unique sous-module irréductible et ce sous-module est $\pi(\psi)$. L'action de $\theta$ sur cette induite qui se déduit naturellement de celle sur $\pi\left(\psi_{-}\right)$induit par restriction l'action de $\theta$ déjà mise sur $\pi(\psi)$.

L'hypothèse est indispensable pour avoir un tel lemme. On suppose d'abord que $A=B$; dans ce cas c'est facile, on applique la définition et les commutations sont évidentes car on a à chaque fois irréductibilité grâce à l'hypothèse. On suppose donc que $A>B$.

L'unicité du sous-module est un calcul de module de Jacquet plus simple que celui fait en 1.12, on ne le refait pas. On écrit la suite d'induites (avec la convention faite dans les définitions si $A-1=B$ pour interpréter la définition de $\pi(\rho, A-2, B, \zeta)$ comme la représentation triviale de $G L(0))$ :

$$
\begin{aligned}
& <\rho||^{\zeta B}, \cdots, \rho||^{\zeta A}>\times \pi\left(\psi_{-}\right) \times<\rho||^{-\zeta A}, \cdots, \rho||^{-\zeta B}>\hookrightarrow \\
& <\rho||^{\zeta B}, \cdots, \rho||^{\zeta A}> \\
& \times<\rho||^{\zeta(B-1), \cdots,-\zeta(A-1)}>\times \pi(\rho, A-2, B, \zeta) \times \pi\left(\psi^{\prime}\right) \times<\rho||^{\zeta(A-1)}, \cdots,\left.\rho\right|^{-\zeta(B-1)}> \\
& \times<\rho||^{-\zeta A}, \cdots,\left.\rho\right|^{-\zeta B}> \\
& \left.\rho\right|^{\zeta B} \times<\rho||^{\zeta(B-1), \cdots,-\zeta(A-1)}>\times<\rho||^{\zeta(B+1)}, \cdots,\left.\rho\right|^{\zeta A}> \\
& \times \pi(\rho, A-2, B, \zeta) \times \pi\left(\psi^{\prime}\right) \\
& \times<\rho||^{-\zeta A}, \cdots, \rho||^{-\zeta(B+1)}>\times<\rho|| \zeta(A-1), \cdots, \rho||^{-\zeta(B-1)}>\times \rho||^{-\zeta B} . \\
& \left.\rho\right|^{\zeta B} \times<\rho||^{\zeta(B-1), \cdots,-\zeta(A-1)}>\times<\rho||^{\zeta(B+1)}, \cdots, \rho||^{\zeta(A-1)}>\times \rho||^{\zeta A} \\
& \times \pi(\rho, A-2, B, \zeta) \times \pi\left(\psi^{\prime}\right) \\
& \times \rho||^{-\zeta A} \times<\rho||^{-\zeta(A-1)}, \cdots, \rho||^{-\zeta(B+1)}>\times<\left.\rho||\right|^{\zeta(A-1)}, \cdots, \rho||^{-\zeta(B-1)}>\times \rho||^{-\zeta B} .
\end{aligned}
$$

Il y a des actions de $\theta$ sur toutes ces induites qui ne dépendent que de l'action de $\theta$ sur $\pi(\rho, A-$ $2, B, \zeta) \times \pi\left(\psi^{\prime}\right)$ et les inclusions sont compatibles aux actions de $\theta$. On sait que $A \geq B+1 \geq 2$ d'où a fortiori $A>1 / 2$ donc les induites ci-dessous sont irréductibles et isomorphes :

$$
\rho||^{-\zeta A} \times \pi(\rho, A-2, B, \zeta) \times \pi\left(\psi^{\prime}\right) \times \rho||^{\zeta A} \simeq \rho||^{\zeta A} \times \pi(\rho, A-2, B, \zeta) \times \pi\left(\psi^{\prime}\right) \times \rho||^{-\zeta A} .
$$

On peut continuer (2) par les isomorphismes :

$$
\begin{gathered}
(2) \simeq \rho||^{\zeta B} \times<\rho||^{\zeta(B-1), \cdots,-\zeta(A-1)}>\times \rho||^{-\zeta A} \\
\times<\rho||^{\zeta(B+1)}, \cdots, \rho||^{\zeta(A-1)}>\times \pi(\rho, A-2, B, \zeta) \times \pi\left(\psi^{\prime}\right) \times<\rho||^{-\zeta(A-1)}, \cdots, \rho||^{-\zeta(B+1)}> \\
\times \rho||^{-\zeta A} \times<\rho||^{\zeta(A-1)}, \cdots, \rho||^{-\zeta(B-1)}>\times \rho||^{-\zeta B} .
\end{gathered}
$$

En admettant le lemme par recurrence, on voit que (2) contient

$$
\begin{gathered}
\rho||^{\zeta B} \times<\rho|| \zeta(B-1), \cdots,-\zeta(A-1)>\times \rho||^{-\zeta A} \times \\
\pi(\rho, A-1, B+1, \zeta) \times \pi\left(\psi^{\prime}\right) \\
\times \rho||^{\zeta A} \times<\rho||^{\zeta(A-1)}, \cdots, \rho||^{-\zeta(B-1)}>\times \rho||^{-\zeta B} .
\end{gathered}
$$

L'action sur l'induite du milieu est celle que nous avons mise sur $\pi\left(\psi^{\prime \prime}\right)$ où $\psi^{\prime \prime}$ est le morphisme qui se déduit de $\psi^{\prime}$ en ajoutant le bloc $(\rho, A-1, B+1, \zeta)$; d'où $\pi\left(\psi^{\prime \prime}\right)=\pi(\rho, A-1, B+1, \zeta) \times \pi\left(\psi^{\prime}\right)$. En tant que représentation de $\tilde{G}(N)$ cette induite contient encore :

$$
<\rho||^{\zeta B}, \cdots, \rho-\zeta A>\times \pi\left(\psi^{\prime \prime}\right) \times<\rho||^{\zeta A}, \cdots,\left.\rho\right|^{-\zeta B}>
$$


Or (3) a encore comme unique sous-module irréductible $\pi(\psi)$ avec la "bonne" action de $\theta$, par définition. L'action de $\theta$ sur $\pi(\psi)$ en tant que sous-module de (1) est donc la même que celle déjà mise sur $\pi(\psi)$. Cela prouve le lemme.

Corollaire. Avec les hypothèses du lemme précédent, soit $C \in[B, A]$ alors $J a c_{\zeta B, \cdots, \zeta C}^{\theta} \pi(\psi)$ est l'unique sous-module irréductible de l'induite

$$
<\rho||^{\zeta(C+1)}, \cdots, \rho||^{\zeta A}>\times \pi\left(\psi_{-}\right) \times<\rho||^{-\zeta A}, \cdots, \rho||^{-\zeta(C+1)}>.
$$

Le lemme précédent permet d'écrire $\pi(\psi)$ comme sous-module de l'induite :

$$
\begin{gathered}
<\rho||^{\zeta B}, \cdots, \rho||^{\zeta C}>\times \\
<<\rho||^{\zeta(C+1)}, \cdots, \rho||^{\zeta A}>\times \pi\left(\psi_{-}\right) \times<\rho||^{-\zeta A}, \cdots, \rho||^{-\zeta(C+1)}>> \\
\times<\rho||^{-\zeta C}, \cdots, \rho||^{-\zeta B}>,
\end{gathered}
$$

les $<>$ extérieurs dans l'induite du milieu disent que l'on prend l'unique sous-module irréductible. Et l'action de $\theta$ prolonge naturellement celle de $\theta$ sur l'induite du milieu. Et le résultat s'en déduit.

\subsection{Remarque}

Soient $B, A$ des demi-entiers tel que $A-B \in \mathbb{N}_{>0}$ et soit $\zeta= \pm 1$. L'induite

$$
<\rho||^{\zeta B}, \cdots, \rho||^{-\zeta A}>\times<\rho||^{\zeta A}, \cdots, \rho||^{-\zeta B}>
$$

est de longueur 2, sans multiplicités et contient comme quotient l'induite irréductible :

$$
<\rho||^{\zeta B}, \cdots, \rho||^{-\zeta B}>\times<\rho||^{\zeta A}, \cdots,\left.\rho\right|^{-\zeta A}>.
$$

Sur (1), on a une action de $\theta$ dès que $\rho \simeq{ }^{\theta} \rho$ et cette action est indépendante du choix d'un opérateur entrelaçant $\rho$ et ${ }^{\theta} \rho$. Sur (2), on vient de mettre une action de $\theta$ sous la même hypothèse, en considérant que (2) correspond au morphisme $\psi$ tel que $\operatorname{Jord}(\psi)=\{(\rho, A, A, \zeta) ;(\rho, B, B, \zeta)\}$.

Remarque : l'action de $\theta$ mise sur (2) est la restriction de celle mise sur (1).

C'est la définition si $B=0$ et si $B$ est entier, avec $B>0$, on se ramène à ce cas en faisant une récurrence : on écrit

$$
\begin{gathered}
<\rho||^{\zeta B}, \cdots, \rho||^{-\zeta A}>\times<\rho||^{\zeta A}, \cdots, \rho||^{-\zeta B}>\hookrightarrow \\
\rho||^{\zeta B} \times<\rho||^{\zeta(B-1)}, \cdots, \rho||^{-\zeta A}>\times<\rho||^{\zeta A}, \cdots, \rho||^{-\zeta(B-1)}>\times \rho||^{-\zeta B} .
\end{gathered}
$$

Par récurrence on admet que la projection de (3) sur

$$
\rho||^{\zeta B} \times<\rho||^{\zeta(B-1)}, \cdots, \rho||^{-\zeta(B-1)}>\times<\rho||^{\zeta A}, \cdots, \rho||^{-\zeta A}>\times \rho||^{-\zeta B}
$$

entrelace le prolongement canonique de l'action de $\theta$ mise sur l'induite du milieu. L'induite (4) contient avec multiplicité 1 la représentation $\tau:=<\rho||^{\zeta B}, \cdots,\left.\rho\right|^{-\zeta B}>\times<\rho||^{\zeta A}, \cdots,\left.\rho\right|^{-\zeta A}>$. Cette représentation est un sous-module de (4) et l'action de $\theta$ que nous y avons mise est la restriction de l'action de $\theta$ sur (4). Par un calcul de module de Jacquet, on vérifie que $\tau$ est l'unique sous-module irréductible de (4). On vérifie aussi que $\tau$ intervient avec multiplicité 1 comme sous-quotient de (3). On a donc construit une application $\theta$-équivariante de (1) dans (4); si elle était nulle, $\tau$ qui intervient dans (1) et dans l'image de (3) dans (4), interviendrait avec multiplicité au moins 2 dans (3). Ceci est exclu et $\tau$ est donc l'image de l'application construite. La $\theta$-équivariance prouve l'assertion.

Il reste à voir le cas où $B$ est demi-entier; la démonstration ci-dessus ramène au cas où $B=1 / 2$, cas que nous allons traiter de façon totalement similaire : on construit comme ci-dessus

$$
<\rho||^{\zeta 1 / 2}, \cdots,\left.\rho\right|^{-\zeta A}>\times<\rho||^{\zeta A}, \cdots,\left.\rho\right|^{-\zeta 1 / 2}>\hookrightarrow
$$




$$
\begin{gathered}
\rho||^{\zeta 1 / 2} \times<\rho||^{-\zeta 1 / 2}, \cdots, \rho||^{-\zeta A}>\times<\rho||^{\zeta A}, \cdots, \rho||^{\zeta 1 / 2}>\times \rho||^{-\zeta 1 / 2} \rightarrow \\
\rho||^{\zeta 1 / 2} \times<\rho||^{\zeta A}, \cdots, \rho||^{-\zeta A}>\times \rho||^{-\zeta 1 / 2} .
\end{gathered}
$$

Le point est de démontrer que cette application est $\theta$-équivariante. Par définition, il n'y a plus qu'à démontrer la $\theta$-équivariance de l'application

$$
<\rho||^{-\zeta 1 / 2}, \cdots, \rho||^{-\zeta A}>\times<\rho||^{\zeta A}, \cdots, \rho||^{\zeta 1 / 2}>\rightarrow<\rho||^{\zeta A}, \cdots, \rho \|\left.\right|^{-\zeta A}>.
$$

Or l'action de $\theta$ mise sur $<\rho||^{\zeta A}, \cdots, \rho \|^{-\zeta A}>$ vient de l'inclusion

$$
<\rho||^{\zeta A}, \cdots, \rho||^{-\zeta A}>\hookrightarrow<\rho||^{\zeta A}, \cdots,\left.\rho\right|^{\zeta 1 / 2}>\times<\rho||^{-\zeta 1 / 2}, \cdots,\left.\rho\right|^{-\zeta A}>.
$$

Mais on a déjà vu que la $\theta$-équivariance résulte de 1.9 en glissant un paramètre $s \in \mathbb{C}$ :

$$
\begin{gathered}
<\rho||^{-\zeta 1 / 2}, \cdots, \rho||^{-\zeta A}>\left.\left\|\left.\right|^{s} \times<\rho||^{\zeta A}, \cdots, \rho\right\|\right|^{-\zeta A}>\|\left.\right|^{-s} \rightarrow \\
<\rho||^{\zeta A}, \cdots,\left.\rho\left\|\left.\right|^{-\zeta A}>\right\|\right|^{-s} \times<\rho||^{-\zeta 1 / 2}, \cdots,\left.\rho\right|^{-\zeta A}>\|^{s} .
\end{gathered}
$$

C'est une application $\theta$-équivariante qui, rendue holomorphe en $s=0$, donne l'égalité des 2 actions de $\theta$ sur $<\rho||^{\zeta A}, \cdots,\left.\rho\right|^{-\zeta A}>$. La fin de la démonstration est comme ci-dessus.

Remarque : supposons que $B=0$; alors pour tout entier $A, \pi(\rho, A, 0,+) \simeq \pi(\rho, A, 0,-)$.

En tant que représentation de $G L\left(a b d_{\rho}, F\right)$ les 2 représentations écrites sont isomorphes. Le problème est l'action de $\theta$. On fait d'abord la démonstration pour $A=1 ; \operatorname{sur} \pi(\rho, 1,0,+), \theta$ agit par $\theta_{+}$obtenu en restreignant l'action naturelle de $\theta$ sur l'induite :

$$
<\rho, \rho||^{-1}>\times<\left.\rho\right|^{+1}, \rho>.
$$

On inclut cette induite dans l'induite :

$$
\rho \times \rho||^{-1} \times \rho||^{+1} \times \rho \simeq \rho \times \rho^{\prime}||^{+1} \times \rho||^{-1} \times \rho,
$$

l'isomorphisme préservant les actions naturelles de $\theta$. L'induite de droite contient encore comme sousmodule l'induite $<\rho, \rho||^{+1}>\times<\left.\rho\right|^{-1}, \rho>$ munie de l'action naturelle de $\theta$ et cette induite contient $\pi(\rho, 1,0,-)$ comme unique sous-module irréductible. Pour conclure, il ne reste plus qu'à remarquer que l'isomorphisme du milieu a identifié $\pi(\rho, 1,0,+)$ et $\pi(\rho, 1,0,-)$.

Le cas où $A>1$ se traite par récurrence pour se ramener au cas ci-dessus.

\section{2 "Résolution" dans le groupe de Grothendieck}

\subsection{Le cas des représentations $\psi$ irréductibles avec inf $(a, b)=2$}

On fixe un triplet $(\rho, a, b)$ et son quadruplet associé $(\rho, A, B, \zeta)$ cf. 1.11. Dans ce paragraphe on a posé $S p(b, S t(a, \rho))=\pi(\rho, A, B, \zeta)$ notation que nous allons utiliser.

Traitons d'abord le cas où $A=B+1$, c'est-à-dire inf $(a, b)=2$. Alors $\pi(\rho, A, B, \zeta)$ est l'unique sous-module irréductible de l'induite (on remplace systématiquement $A$ par $B+1$ ) :

$$
<\rho||^{\zeta B}, \cdots,\left.\rho\right|^{-\zeta(B+1)}>\times<\rho||^{\zeta(B+1)}, \cdots,\left.\rho\right|^{-\zeta B}>.
$$

Cette induite est en fait de longueur 2 avec comme quotient irréductible la représentation induite

$$
<\rho||^{\zeta(B+1)}, \cdots, \rho||^{-\zeta(B+1)}>\times<\rho||^{\zeta B}, \cdots,\left.\rho\right|^{-\zeta B}>.
$$

On remarque d'ailleurs que (2) n'est autre que l'induite $\pi(\rho, A=B+1, B+1, \zeta) \times \pi(\rho, B, B, \zeta)$. Il faut comparer l'action de $\theta$ que nous avons mis sur (2) avec celle que l'on obtient en passant au quotient l'action de $\theta$ mise sur (1). On a démontré en 1.14 que ces 2 actions coïncidaient . Ainsi ici, $\pi(\rho, A, B, \zeta)$ est la différence des 2 induites celle écrite en (1) moins celle écrite en (2), différence dans le groupe de Grothendieck. 


\subsection{Le cas des représentations $\psi$ irréductibles}

Dans ce paragraphe on va généraliser la formule de 2.1. On reprend les notations $(\rho, A, B, \zeta)$ mais on suppose ici $A>B+1$. On pose :

$$
\begin{gathered}
\tilde{\pi}(\rho, A, B, \zeta):= \\
\oplus_{C \in] B, A]}(-1)^{A-C}<\rho||^{\zeta B}, \cdots, \rho||^{-\zeta C}>\times J a c_{\zeta(B+2), \cdots, \zeta C}^{\theta} \pi(\rho, A, B+2, \zeta) \times<\rho||^{\zeta C}, \cdots, \rho||^{-\zeta B}> \\
\oplus(-1)^{[(A-B+1) / 2]} \pi(\rho, A, B+1, \zeta) \times \pi(\rho, B, B, \zeta),
\end{gathered}
$$

où par convention pour $C=B+1, J a c_{\zeta(B+2), \cdots, \zeta C}^{\theta} \pi(\rho, A, B+2, \zeta)$ est simplement $\pi(\rho, A, B+2, \zeta)$. Toutes les représentations intervenant ont été munies d'une action de $\theta$ et $\tilde{\pi}(\rho, A, B, \zeta)$ est donc un élément du groupe de Grothendieck des représentations de $\tilde{G}\left(a b d_{\rho}\right)$. Il n'est pas vrai en général que $\pi(\rho, A, B, \zeta)=\tilde{\pi}(\rho, A, B, \zeta)$ mais on va montrer :

Proposition : Dans sa description comme combinaison linéaire de représentations irréductibles de $\tilde{G}\left(a b d_{\rho}\right), \tilde{\pi}(\rho, A, B, \zeta)$ contient exactement une représentation irréductible qui reste irréductible quand on la restreint à $G L\left(a b d_{\rho}, F\right)$; cette représentation est $\pi(\rho, A, B, \zeta)$ et elle intervient avec le coefficient +1 . Plus élégamment, pour tout $g \in G L(n, F) \theta$-semi-simple et régulier,

$$
\operatorname{tr}(\tilde{\pi}(\rho, A, B, \zeta))(g, \theta)=\operatorname{tr} \pi(\rho, A, B, \zeta)(g, \theta) .
$$

\subsubsection{Description des représentations dans la classification de Zelevinsky}

Avant de commencer la démonstration, décrivons, pour $C \in] B, A]$ la représentation $J a c_{\zeta(B+2), \cdots, \zeta C}^{\theta} \pi(\rho$, $A, B+2, \zeta)$ dans la classification de Zelevinsky. Notons $\mathcal{T}_{A, B, \zeta}$ le tableau suivant dont les lignes sont des segments décroissants si $\zeta=+$ et croissants si $\zeta=-$ et les colonnes sont des segments ayant les propriétés de croissance opposées, en les considérant du haut vers le bas.

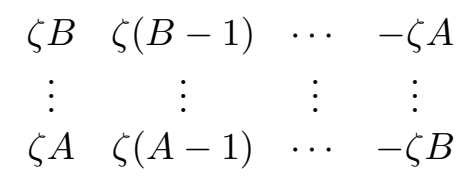

Ce tableau a $\sup (a, b)$ colonnes et $\inf (a, b)$ lignes.

On peut considérer le tableau analogue en remplaçant $B$ par $B+2$ et en gardant $A$ et $\zeta$, c'est-à-dire $\mathcal{T}_{(A, B+2, \zeta)}:$

$$
\begin{array}{cccc}
\zeta(B+2) & \zeta(B+1) & \cdots & -\zeta A \\
\vdots & \vdots & \vdots & \vdots \\
\zeta A & \zeta(A-1) & \cdots & -\zeta(B+2)
\end{array}
$$

Ce tableau a $\sup (a, b)+2$ colonnes et $\inf (a, b)-2$ lignes. Soit $C \in] B, A]$; on suppose même que $C \geq B+2$ pour qu'il y ait quelque chose à décrire. Et notons $\mathcal{T}_{C}$ le tableau qui s'obtient à partir de celui écrit ci-dessus mais en enlevant les $(C-B-1)$ premiers éléments de la première colonne et les $(C-B-1)$ derniers éléments de la dernière colonne; en particulier, on n'enlève rien si $C=B+1$. Ecrivons ce que l'on obtient dans le cas où $A+B \geq 2 C$

$$
\begin{array}{cccc}
\zeta(B+1) & \ldots & \ldots & -\zeta A \\
\vdots & \vdots & \vdots & \vdots \\
\zeta(C) & \cdots & \ldots & -\zeta(A-C+B+1) \\
\vdots & \vdots & \vdots & \vdots \\
\ldots & \ldots & -\zeta C & -\zeta(C+1) \\
\vdots & \vdots & \vdots & \\
\ldots & \cdots & -\zeta(B+1) &
\end{array}
$$


Dans le cas opposé, c'est-à-dire $A+B<2 C$, on obtient :

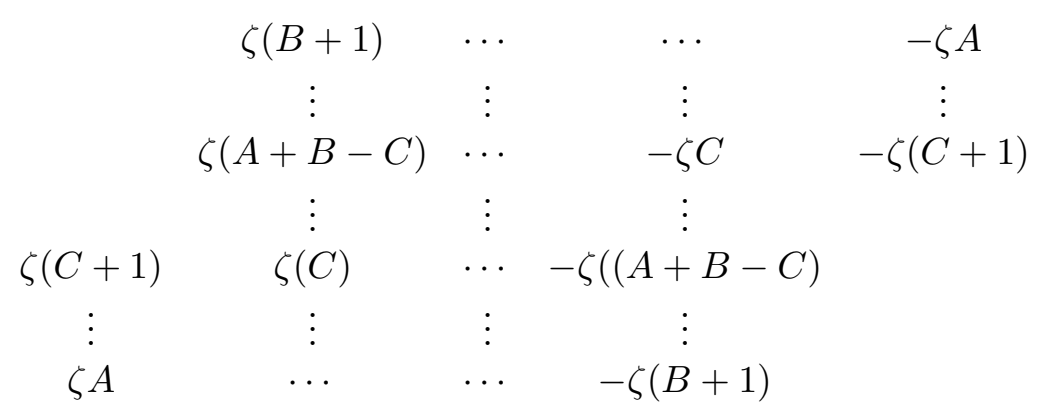

Quel que soit le tableau écrit ci-dessus, la classification de Zelevinsky montre qu'il existe exactement une unique représentation irréductible sous-module de l'induite associée aux multi-segments formés par l'ensemble des lignes mis dans l'ordre des lignes (du haut vers le bas). On la note $\sigma_{\mathcal{T}}$ où $\mathcal{T}$ est le tableau correspondant. Avec un tableau écrit comme ci-dessus avec les lignes formées de segments et les colonnes formées aussi de segments mais avec la propriété de croissance opposée à celle des lignes, on vérifie que $\sigma_{\mathcal{T}}$ est aussi l'unique sous-représentation irréductible de l'induite associée aux multi-segments formés par l'ensemble des colonnes mis dans l'ordre des colonnes (de gauche vers la droite). On a en tant que représentation du $G L$ convenable

$$
\begin{gathered}
\pi(\rho, A, B, \zeta)=\sigma_{\mathcal{T}_{A, B, \zeta}}, \quad \pi(\rho, A, B+2, \zeta)=\sigma_{\mathcal{T}_{A, B+2, \zeta}} \\
J a c_{\zeta(B+2), \cdots, \zeta C}^{\theta} \pi(\rho, A, B+2, \zeta)=\sigma_{\mathcal{T}_{C}} .
\end{gathered}
$$

De plus, avec la description ci-dessus, on a $J a c_{x} J a c_{\zeta(B+2), \cdots, \zeta C}^{\theta} \pi(\rho, A, B+2, \zeta) \neq 0$ entraîne que $x$ est à la fois un début de ligne et un début de colonne, c'est-à-dire ici, nécessairement $x=\zeta(B+1)$ ou $x=\zeta(C+1)$. On peut aussi avoir l'action de $\theta:$ pour $\pi(\rho, A, B, \zeta)$ la définition dit qu'il faut privilégier la représentation comme sous-module en utilisant les segments formés par les lignes. Mais 1.13 et 1.14 montrent que l'on obtient la même action de $\theta$ en utilisant les colonnes. Avec les mêmes références, on démontre le même résultat pour $J a c_{\zeta(B+2), \cdots, \zeta C}^{\theta} \pi(\rho, A, B+2, \zeta)$.

\subsubsection{Propriété des modules de Jacquet et preuve}

Dans tout ce qui suit, on fixe $\tilde{\pi}$ une représentation irréductible de $\tilde{G}\left(a b d_{\rho}\right)$ qui intervient avec un coefficient non nul dans la description de $\tilde{\pi}(\rho, A, B, \zeta)$.

Lemme : (i) Soit $x \in \mathbb{R}$. On a $J a c_{x} \tilde{\pi}=0$ pour tout $x \notin[\zeta B, \zeta A]$ et on a $J a c_{x, x} \tilde{\pi}=0$ pour tout $x$.

(ii) On suppose ici que la restriction de $\tilde{\pi}$ à $G L\left(a b d_{\rho}, F\right)$ est irréductible; alors pour tout $x \in \mathbb{R}$, $J a c_{x}^{\theta} \tilde{\pi}=0$ sauf éventuellement pour $x=\zeta B$.

(iii) La proposition 2.2 est vraie c'est-à-dire que $\tilde{\pi}(\rho, A, B, \zeta)$ contient une unique représentation irréductible dont la restriction à $G L\left(a b d_{\rho}, F\right)$ est irréductible, elle intervient avec coefficient 1 et est exactement $\pi(\rho, A, B, \zeta)$.

Comme $\tilde{\pi}$ est irréductible, soit $\tilde{\pi}$ est l'induite $\pi(\rho, A, B+1, \zeta) \times \pi(\rho, B, B, \zeta)$ soit il existe $C \in] B, A]$ tel que $\tilde{\pi}$ est un sous-quotient irréductible de l'induite

$$
\sigma_{C}:=<\rho||^{\zeta B}, \cdots, \rho||^{-\zeta C}>\times J a c_{\zeta(B+2), \cdots, \zeta C}^{\theta} \pi(\rho, A, B+2, \zeta) \times<\rho||^{\zeta C}, \cdots, \rho||^{-\zeta B}>.
$$

Les 2 propriétés ne sont pas exclusives l'une de l'autre.

On vérifie que $J a c_{x}(\pi(\rho, A, B+1, \zeta) \times \pi(\rho, B, B, \zeta))=0$ sauf exactement pour $x=\zeta B$ et $x=$ $\zeta(B+1)$ et on vérifie aussi aisément que $\operatorname{Jac}_{x, x}(\pi(\rho, A, B+1, \zeta) \times \pi(\rho, B, B, \zeta))=0$ pour tout $x$ réel. Supposons que $\tilde{\pi}$ est un sous-quotient irréductible de $\sigma_{C}$ pour $\left.\left.C \in\right] B, A\right]$. Si $J a c_{x} \tilde{\pi} \neq 0$, nécessairement soit $x=\zeta B$, soit $x=\zeta C$ soit $J a c_{x} J a c_{\zeta(B+2), \cdots, \zeta C}^{\theta} \pi(\rho, A, B+2, \zeta) \neq 0$. Pour étudier cette dernière possibilité on utilise la réalisation de $J a c_{\zeta(B+2), \cdots, \zeta C}^{\theta} \pi(\rho, A, B+2, \zeta)$ comme $\sigma_{\mathcal{T}_{C}}$; on a vu que la non 
nullité nécessite que soit $x=\zeta(B+1)$ soit $x=\zeta(C+1)$ avec les 2 cas particuliers suivant : si $C=B+1$ seul la valeur $x=\zeta(B+2)$ donne quelque chose de non nul et si $C=A$ seul $x=\zeta(B+1)$ donne quelque chose de non nul. On vérifie alors aisément, dans tous les cas, que $J a c_{x, x} \sigma_{C}=0$ pour toute valeur de $x$. Cela termine la preuve de (i).

Prouvons (ii); la seule difficulté peut venir d'un réel $x$ vérifiant $J a c_{x} \tilde{\pi} \neq 0$ et d'après (i) cela entraîne que $x \in[\zeta B, \zeta A]$. Il faut donc montrer que pour tout $C \in] B, A], J a c_{\zeta C}^{\theta} \tilde{\pi}=0$.

On va d'abord traiter le cas où $C \in[B+2, A]$ où on va en fait montrer que $\operatorname{Jac}_{\zeta C}^{\theta} \tilde{\pi}(\rho, A, B, \zeta)=0$. On pose $x=\zeta C$. Montrons d'abord comment cela permettra de conclure; les arguments ci-dessous ne suppose que $\zeta x \in] B, A]$, c'est-à-dire $C \in] B, A]$.

Faisons remarquer tout de suite que, pour les valeurs que nous considérons $x \neq 0$. Soit $\pi^{\prime}$ une représentation irréductible de $\tilde{G}\left(a b d_{\rho}\right)$ intervenant dans $\tilde{\pi}(\rho, A, B, \zeta)$ et telle que $J a c_{x}^{\theta} \pi^{\prime} \neq 0$; on considère la restriction de $\pi^{\prime}$ à $G L\left(a b d_{\rho}, F\right)$ et son module de Jacquet relativement au parabolique de Levi $G L\left(d_{\rho}\right) \times G L\left((a b-2) d_{\rho}, F\right) \times G L\left(d_{\rho}\right)$. Par hypothèse la projection de cette représentation sur la composante où le premier facteur de $G L\left(d_{\rho}\right)$ agit par $\rho \|^{x}$ et le troisième facteur agit par $\left.\rho\right|^{-x}$ de façon isotypique est non nulle. Par réciprocité de Frobenius, il existe une représentation irréductible $\pi_{0}$ de $G L\left((a b-2) d_{\rho}, F\right)$ et une inclusion d'une des composantes irréductibles de la restriction de $\pi^{\prime}$ à $G L\left(a b d_{\rho}, F\right)$ dans l'induite $\left.\rho\right|^{x} \times \pi_{0} \times\left.\rho\right|^{-x}$. On note $\pi_{0}^{\prime}$ une composante irréductible de la restriction de $\pi^{\prime}$ à $G L\left(a b d_{\rho}, F\right)$ incluse dans cette induite.

On calcule $\operatorname{Jac}_{x}^{\theta}\left(\left.\rho\right|^{x} \times \pi_{0} \times\left.\rho\right|^{-x}\right)=\pi_{0}$ : pour cela on remarque d'abord que si $J a c_{x} \pi_{0} \neq 0$, on aurait aussi $J a c_{x, x} \pi_{0}^{\prime} \neq 0$ et certainement aussi $J a c_{x, x} \pi^{\prime} \neq 0$ et une contractiction avec (i). Ainsi $J a c_{x} \pi_{0}=0$. Comme $x \neq 0$, on a aussi $J a c_{x}\left(\pi_{0} \times \rho||^{-x}\right)=0$. D'où l'assertion.

On déduit de cette assertion que l'induite $\left.\rho\right|^{x} \times \pi_{0} \times\left.\rho\right|^{-x}$ a un unique sous- $G L\left(a b d_{\rho}, F\right)$-module irréductible qui ici est nécessairement $\pi_{0}^{\prime}$. L'unicité assure aussi que $\pi_{0}^{\prime}$ est $\theta$-invariante si et seulement si $\pi_{0}$ l'est. Supposons que $\pi_{0}$ est $\theta$-invariante; on a donc $\pi_{0}^{\prime}=\pi^{\prime}$ comme représentation de $G L\left(a b d_{\rho}, F\right)$. Mais $\pi_{0}^{\prime}$ hérite alors d'une action de $\theta$ qui donne une action de $\theta$ sur $\pi_{0}$ uniquement déterminée. Par contre si $\pi_{0}$ n'est pas $\theta$-invariante $\pi^{\prime}$ et l'action de $\theta$ sur $\pi^{\prime}$ sont uniquement déterminés par $\pi_{0}^{\prime}$ et donc par $\pi_{0}$. Donc dans les 2 cas, la seule hypothèse $J a c_{x}^{\theta} \pi^{\prime} \neq 0$ (ajoutée à l'hypothèse que $\pi^{\prime}$ intervient dans $\tilde{\pi}(\rho, A, B, \zeta))$ entraîne que $J a c_{x}^{\theta} \pi^{\prime}$ est irréductible comme $\tilde{G}\left((a b-2) d_{\rho}\right)$-module et détermine uniquement $\pi^{\prime}$. Cela prouve qu'il ne peut y avoir de simplification dans le groupe de Grothendieck pour $\tilde{G}\left((a b-2) d_{\rho}\right)$ entre des $J a c_{x}^{\theta} \pi^{\prime}$ faisant intervenir des $\pi^{\prime}$ différents, ou encore que $J a c_{x}^{\theta} \tilde{\pi}(\rho, A, B, \zeta)=0$ entraîne que $J a c_{x}^{\theta} \pi^{\prime}=0$ pour tout $\pi^{\prime}$ représentation irréductible de $\tilde{G}$ intervenant avec un coefficient non nul dans $\pi(\rho, \tilde{A, B}, \zeta)$.

Il nous reste à montrer que pour tout $C \in](B+1), A], J a c_{\zeta C}^{\theta} \tilde{\pi}(\rho, A, B, \zeta)=0$. Il y a exactement 2 termes dans la définition de $\tilde{\pi}(\rho, A, B, \zeta)$ qui vérifient simplement $J a c_{\zeta C} \neq 0$, il s'agit, d'après ce que l'on a vu dans la preuve de (i) de $\sigma_{C}$ et $\sigma_{C-1}$. On calcule

$$
J a c_{\zeta C}^{\theta} \sigma_{C}=<\rho||^{\zeta B}, \cdots, \rho||^{-\zeta(C-1)}>\times J a c_{\zeta(B+2), \cdots, \zeta C}^{\theta} \pi(\rho, A, B+2, \zeta) \times<\rho||^{\zeta(C-1)}, \cdots,\left.\rho\right|^{-\zeta B}>;
$$

Pour voir l'action de $\theta$ sur ce module de Jacquet, on considère d'abord l'inclusion :

$$
<\rho||^{\zeta B}, \cdots, \rho||^{-\zeta(C-1)}>\times \rho||^{-\zeta C} \times J a c_{\zeta(B+2), \cdots, \zeta C}^{\theta} \pi(\rho, A, B+2, \zeta) \times \rho||^{\zeta C} \times<\rho||^{\zeta(C-1)}, \cdots, \rho||^{-\zeta B}>.
$$

C'est une inclusion qui respecte les actions naturelles de $\theta$. Le calcul de $J a c^{\theta}$ se fait avec la filtration de Bernstein Zelevinsky qui provient des doubles classes du type $P_{0} w P_{0}$ pour $w$ parcourant un bon ensemble de matrices de permutations. L'action de $\theta$ respecte cette filtration à condition de regrouper $w$ avec ${ }^{\theta} w$; le terme qui nous intéresse correspond à un unique $w$ dont la double classe est invariante sous l'action de $\theta$. L'action de $\theta$ obtenue sur $J a c_{\zeta C}^{\theta}$ est l'action obtenue sur $J a c_{\zeta C}^{\theta}$ appliqué à

$<\rho||^{\zeta B}, \cdots,\left.\rho\right|^{-\zeta(C-1)}>\times \rho||^{\zeta C} \times J a c_{\zeta(B+2), \cdots, \zeta C}^{\theta} \pi(\rho, A, B+2, \zeta) \times\left.\rho\right|^{-\zeta C} \times<\rho||^{\zeta(C-1)}, \cdots, \rho||^{-\zeta B}>$,

car on peut remplacer l'induite du milieu par son unique sous-module irréductible. Mais cette dernière induite est isomorphe à :

$\left.\rho\right|^{\zeta C} \times<\rho||^{\zeta B}, \cdots,\left.\rho\right|^{-\zeta(C-1)}>\times J a c_{\zeta(B+2), \cdots, \zeta C}^{\theta} \pi(\rho, A, B+2, \zeta) \times<\rho||^{\zeta(C-1)}, \cdots, \rho||^{-\zeta B}>\times \rho||^{-\zeta C}$, 
car $C \neq B+1$. L'action de $\theta$ sur $J a c_{\zeta C}^{\theta}$ de l'induite ci-dessus est l'action naturelle sans aucune ambiguïté. On a aussi

$$
\begin{gathered}
J_{a c_{\zeta C}}^{\theta} \sigma_{C-1}= \\
<\rho||^{\zeta B}, \cdots,\left.\rho\right|^{-\zeta(C-1)}>\times J a c_{\zeta C}^{\theta} J a c_{\zeta(B+2), \cdots, \zeta(C-1)}^{\theta} \pi(\rho, A, B+2, \zeta) \times<\rho||^{\zeta(C-1)}, \cdots,\left.\rho\right|^{-\zeta B}>.
\end{gathered}
$$

Pour voir l'action de $\theta$ le plus simple est d'écrire $J a c_{\zeta(B+2), \cdots, \zeta(C-1)} \pi(\rho, A, B+2, \zeta)$ comme l'unique sous-module irréductible de l'induite :

$$
\left.\rho\right|^{\zeta C} \times J a c_{\zeta(B+2), \cdots, \zeta C}^{\theta} \pi(\rho, A, B+2, \zeta) \times \rho \|^{-\zeta C}
$$

cette inclusion respecte les actions de $\theta$ par la définition même. Et l'action de $\theta$ sur (2) est l'action naturelle.

Ces termes (1) et (2) sont donc exactement les mêmes, action de $\theta$ comprise mais l'un intervient avec le signe $(-1)^{A-C}$ tandis que l'autre intervient avec le signe $(-1)^{A-C+1}$; ils s'éliminent donc, prouvant l'assertion cherchée.

Considérons pour finir le cas $x=\zeta(B+1)$; ici on démontre moins que pour $C \in] B+1, A]$ c'est-àdire on démontre seulement que pour $\tilde{\pi}$ une représentation irréductible de $\tilde{G}\left(a b d_{\rho}\right)$ intervenant dans $\tilde{\pi}(\rho, A, B, \zeta)$ dont la restriction à $G L\left(a b d_{\rho}, F\right)$ est irréductible, $J a c_{\zeta(B+1)}^{\theta} \tilde{\pi}=0$. Fixons un tel $\tilde{\pi}$ et pour éviter les confusions, on note $\pi$ la restriction de $\tilde{\pi}$ à $G L\left(a b d_{\rho}\right)$. Le support cuspidal de $\pi$ est un ensemble de représentations cuspidales de la forme $\rho \|^{z}$ avec $z$ réel; on note $\mathcal{E}_{\pi}$ cet ensemble de nombres réels comptés avec multiplicité. Pour un ordre convenable sur $\mathcal{E}$, il existe une inclusion :

$$
\pi \hookrightarrow \times\left._{z \in \mathcal{E} \rho} \rho\right|^{z}
$$

Le réel $-\zeta A$ est soit le plus petit élément de $\mathcal{E}$ soit le plus grand (cela dépend de la valeur de $\zeta$ ). De façon formelle on montre qu'il existe $x_{0} \in \mathcal{E}$ tel que $\left[x_{0},-\zeta A\right]$ soit un segment et qu'il existe un ordre sur $\mathcal{E}$ tel que (1) soit encore vrai mais tel que les premiers éléments de $\mathcal{E}$ soient précisément les éléments du segment $\left[x_{0},-\zeta A\right]$. En effet fixons sur $\mathcal{E}$ un ordre tel que la place de $-\zeta A$ soit la plus petite possible. On note $x_{0}$ le premier élément de $\mathcal{E}$ et on va vérifier qu'il convient, c'est-à-dire que $\left[x_{0},-\zeta A\right]$ est un segment. On note $t$ le nombre d'éléments de $\mathcal{E}$ plus petit ou égaux (pour l'ordre fixé) à $-\zeta A$, c'est-à-dire que les $t$ premiers éléments de $\mathcal{E}$ sont $x_{i}$ pour $i \in[1, t]$ avec $x_{t}=-\zeta A$. On montre par récurrence décroissante sur $i$ que $\left[x_{i},-\zeta A\right]$ est un segment et que (1) se précise en une inclusion :

$$
\pi \hookrightarrow \times_{j<i} \rho||^{x_{j}} \times<\rho||^{x_{i}}, \cdots, \rho\left\|^{-\zeta A}>\times_{z \in \mathcal{E}-\left\{x_{1}, \cdots, x_{t}\right\}} \rho\right\|^{z} .
$$

Ceci est trivialement vrai si $i=t$. Admettons le pour $i$ et montrons le pour $i-1$; la représentation induite :

$$
\rho||^{x_{i-1}} \times<\rho||^{x_{i}}, \cdots, \rho||^{-\zeta A}>
$$

est soit irréductible soit de longueur 2 ; elle ne peut être irréductible, sinon on pourrait remplacer $t$ par $t-1$ en faisant commuter $x_{i-1}$ au dessus de $<\rho||^{x_{i}}, \cdots,\left.\rho\right|^{-A}>$. Elle est donc de longueur 2 , c'est-à-dire soit $x_{i-1}=x_{i}+\zeta$ soit $x_{i-1}=-\zeta A-\zeta$; la dernière éventualité est impossible par extrémalité de $-\zeta A$. Donc $\left[x_{i-1},-\zeta A\right]$ est un segment et l'inclusion (2) se factorise soit par

$$
\pi \hookrightarrow \times_{j<i-1} \rho||^{x_{j}} \times<\rho||^{x_{i-1}}, \cdots, \rho||^{-\zeta A}>\times\left._{z \in \mathcal{E}-\left\{x_{1}, \cdots, x_{t}\right\}} \rho\right|^{z}
$$

soit par

$$
\pi \hookrightarrow \times_{j<i-1} \rho||^{x_{j}} \times<\rho||^{x_{i}}, \cdots, \rho\left\|\left.\right|^{-\zeta A}>\times \rho||^{x_{i}} \times_{z \in \mathcal{E}-\left\{x_{1}, \cdots, x_{t}\right\}} \rho\right\|^{z} .
$$

Mais (4) est contraire à la minimalité de $t$ et c'est donc (3) qui prévaut, montrant ainsi notre assertion. Ainsi, il existe $x_{0} \in \mathcal{E}$ et une représentation irréductible $\pi_{0}^{\prime}$ convenable telle que

$$
\pi \hookrightarrow<\rho||^{x_{0}}, \cdots, \rho||^{-\zeta A}>\times \pi_{0}^{\prime} .
$$

Grâce à (i), on sait que $x_{0} \in[\zeta B, \zeta A]$. 
Supposons d'abord que $x_{0}=\zeta B$; dans ce cas, on va très facilement montrer que $\tilde{\pi}=\pi(\rho, A, B, \zeta)$ et donc en particulier $J a c_{\zeta(B+1)} \tilde{\pi}=0$, ce que l'on cherche. En effet, supposons d'abord qu'il existe $C \in] B, A]$ tel que $\tilde{\pi}$ soit un sous-quotient de

$$
\sigma_{C}:=<\rho||^{\zeta B}, \cdots,\left.\rho\right|^{-\zeta C}>\times J a c_{\zeta(B+2), \cdots, \zeta C}^{\theta}(\pi(\rho, A, B+2, \zeta)) \times<\rho||^{\zeta C}, \cdots,\left.\rho\right|^{-\zeta B}>.
$$

On calcule $J a c_{\zeta B, \cdots-\zeta A} \sigma_{C}$ par les formules standard en se souvenant que $J a c_{x} J a c_{\zeta(B+2), \cdots, \zeta C}^{\theta}(\pi(A, B+$ $2, \zeta))=0$ sauf éventuellement si $x=\zeta(B+1)$ ou $x=\zeta(C+1)$. Or ni $\zeta(B+1)$ ni $\zeta(C+1)$ ni $\zeta C$ ne sont dans le segment $[\zeta B,-\zeta A]$. Ainsi

$$
\begin{gathered}
J a c_{\zeta B, \cdots-\zeta A} \sigma_{C}=\left(J a c_{\zeta B, \cdots,-\zeta A}<\rho||^{\zeta B}, \cdots,\left.\rho\right|^{-\zeta C}>\right) \times \\
J a c_{\zeta(B+2), \cdots, \zeta C}^{\theta}(\pi(A, B+2, \zeta)) \times<\rho||^{\zeta C}, \cdots,\left.\rho\right|^{-\zeta B}>
\end{gathered}
$$

Et cela est nul sauf si $C=A$ où cela vaut $J a c_{\zeta(B+2), \cdots, \zeta A}^{\theta}(\pi(\rho, A, B+2, \zeta)) \times<\rho||^{\zeta A}, \cdots,\left.\rho\right|^{-\zeta B}>$. On vérifie que $J a c_{\zeta(B+2), \cdots, \zeta A}^{\theta}(\pi(\rho, A, B+2, \zeta))=\pi(\rho, A-1, B+1, \zeta)$ en particulier est irréductible et on obtient l'inclusion :

$$
\tilde{\pi} \hookrightarrow<\rho||^{\zeta B}, \cdots, \rho||^{-\zeta A}>\times \pi(\rho, A-1, B+1, \zeta) \times<\rho||^{\zeta A}, \cdots,\left.\rho\right|^{-\zeta B}>.
$$

Ainsi $\tilde{\pi}$ est l'unique sous-module irréductible de cette induite avec l'action de $\theta$ qui provient de l'action sur l'induite. Par définition, $\tilde{\pi}=\pi(\rho, A, B, \zeta)$. Il reste à voir le cas où $\tilde{\pi}$ est un sous-quotient de $\pi(\rho, A, B+1, \zeta) \times<\left.\rho\right|^{\zeta B}, \cdots,\left.\rho\right|^{-\zeta B}>$. Ici on sait que $J a c_{x} \pi(\rho, A, B+1, \zeta)=0$ sauf pour $x=$ $\zeta(B+1)$ et il est facile de voir que cela entraîne que

$$
\begin{aligned}
& J a c_{\zeta B, \cdots,-\zeta A}\left(\pi(\rho, A, B+1, \zeta) \times<\rho||^{\zeta B}, \cdots,\left.\rho\right|^{-\zeta B}>\right)= \\
& \pi(\rho, A, B+1, \zeta) \times J a c_{\zeta B, \cdots,-\zeta A}<\rho||^{\zeta B}, \cdots,\left.\rho\right|^{-\zeta B}>=0 .
\end{aligned}
$$

Cela termine le cas où $x_{0}=\zeta B$.

Supposons donc maintenant que $\left.\left.x_{0} \in\right] \zeta B, \zeta A\right]$. On écrit donc :

$$
\pi \hookrightarrow<\rho||^{x_{0}}, \cdots, \rho||^{-\zeta A}>\times \pi_{0}^{\prime},
$$

pour $\pi_{0}^{\prime}$ irréductible convenable. On utilise le fait que $\pi$ est $\theta$-invariante et on obtient une autre inclusion :

$$
\pi \hookrightarrow{ }^{\theta} \pi_{0}^{\prime} \times<\rho||^{\zeta A}, \cdots, \rho||^{-x_{0}}>.
$$

On applique $J a c_{x_{0}, \cdots,-\zeta A}$ à l'induite de droite et comme $\zeta A \notin\left[x_{0},-\zeta A\right]$ cela vaut

$$
J a c_{x_{0}, \cdots-\zeta A}{ }^{\theta} \pi_{0}^{\prime} \times<\rho||^{\zeta A}, \cdots,\left.\rho\right|^{-x_{0}}>.
$$

Par exactitude du foncteur de Jacquet, on obtient une inclusion :

$$
J a c_{x_{0}, \cdots-\zeta A} \pi=\pi_{0}^{\prime} \hookrightarrow J a c_{x_{0}, \cdots-\zeta A}{ }^{\theta} \pi_{0}^{\prime} \times<\rho||^{\zeta A}, \cdots,\left.\rho\right|^{-x_{0}}>.
$$

Il existe donc un sous-quotient irréductible $\pi_{1}^{\prime \prime}$ de $J a c_{x_{0}, \cdots,-\zeta A} \pi_{0}^{\prime}$ tel que en posant $\pi_{1}^{\prime}:={ }^{\theta} \pi_{1}^{\prime \prime}$

$$
\pi_{0}^{\prime} \hookrightarrow \pi_{1}^{\prime} \times<\rho||^{\zeta A}, \cdots,\left.\rho\right|^{-x_{0}}>
$$

En remontant, on obtient :

$$
\pi \hookrightarrow<\rho||^{x_{0}}, \cdots,\left.\rho\right|^{-\zeta A}>\times \pi_{1}^{\prime} \times<\rho||^{\zeta A}, \cdots, \rho||^{-x_{0}}>.
$$

On vérifie que $\pi_{1}^{\prime}=J a c_{x_{0}, \cdots,-\zeta A}^{\theta} \pi$ et hérite d'une action de $\theta$ provenant de l'action de $\theta$ sur $\tilde{\pi}$, on note alors $\pi_{1}$ la représentation ainsi étendue. Avec cette action, on obtient l'inclusion :

$$
\tilde{\pi} \hookrightarrow<\rho||^{x_{0}}, \cdots,\left.\rho\right|^{-\zeta A}>\times \pi_{1} \times<\rho||^{\zeta A}, \cdots, \rho||^{-x_{0}}>
$$


Grâce à ce que l'on a déjà démontré, $x_{0}=\zeta(B+1)$. De plus, $\tilde{\pi}$ est uniquement déterminée par $\pi_{1}$ et l'inclusion (5). Pour démontrer qu'il n'existe pas de tel $\tilde{\pi}$ intervenant dans $\tilde{\pi}(\rho, A, B, \zeta)$, il suffit donc de démontrer que $J a c_{\zeta(B+1), \cdots,-\zeta A}^{\theta} \tilde{\pi}(\rho, A, B, \zeta)$ ne contient pas de représentation irréductible de $\left.\tilde{G}(a b-2(\sup (a, b)+1)) d_{\rho}\right)$ dont la restriction au GL correspondant reste irréductible.

On reprend la notation $\sigma_{C}$ introduite ci-dessus. On va avoir besoin des formules ci-dessous : soit $C \in] B+1, A[$ (pour que l'ensemble soit non vide, il faut $A>B+2$ ) et $x \in[\zeta(B+1),-\zeta A[$, alors

$$
J a c_{y} J a c_{\zeta(B+1), \cdots, x}^{\theta} J a c_{\zeta(B+2), \cdots, \zeta C}^{\theta} \pi(\rho, A, B+2, \zeta)=0,
$$

pour tout $y \in] x-\zeta,-\zeta A]$ et

$$
J a c_{\zeta(B+1), \cdots,-\zeta A}^{\theta} J a c_{\zeta(B+2), \cdots, \zeta C}^{\theta} \pi(\rho, A, B+2, \zeta)=J a c_{\zeta(B+3), \cdots, \zeta C}^{\theta} \pi(\rho, A-1, B+3, \zeta),
$$

cette égalité étant compatible aux actions de $\theta$ et si $A=B+3$ le terme de droite ci-dessus est la représentation triviale de $\tilde{G}(0)$. Pour les vérifier on utilise la représentation de $J a c_{\zeta(B+2), \cdots, \zeta C}^{\theta} \pi(\rho, A, B+$ $2, \zeta)$ donnée à la fin de 2.1; en calculant $J a c_{\zeta(B+1), \cdots x}^{\theta}$ on enlève du tableau une partie (tout si $x=-\zeta A$ ) de la première et symétriquement de la dernière ligne. Et ensuite on voit sur le tableau les valeurs de $y$ pour lesquelles $J a c_{y}$ du résultat peut être non nul, $y$ doit au moins être un début de ligne. L'action de $\theta$ a été précisée en loc.cit, d'où nos assertions. Cela entraîne que pour $C \in] B+1, A[$

$$
\begin{gathered}
J a c_{\zeta(B+1), \cdots,-\zeta A}^{\theta} \sigma_{C}= \\
<\rho||^{\zeta B}, \cdots, \rho||^{-\zeta C}>\times J a c_{\zeta(B+3), \cdots, \zeta C}^{\theta} \pi(\rho, A-1, B+3, \zeta) \times<\rho||^{\zeta C}, \cdots,\left.\rho\right|^{-\zeta B}>.
\end{gathered}
$$

Pour $C=B+1$, on a tout simplement $J a c_{x} \pi(\rho, A, B+2, \zeta)=0$ pour tout $x \in[\zeta B,-\zeta(B+1)]$ et

$$
J a c_{\zeta(B+1), \cdots,-\zeta A^{\theta}}^{\sigma_{B+1}}=J a c_{-\zeta(B+2), \cdots,-\zeta A} \pi(\rho, A, B+2, \zeta)=0 .
$$

Pour $C=A, J a c_{\zeta(B+2), \cdots, \zeta A}^{\theta} \pi(\rho, A, B+2, \zeta)=\pi(\rho, A-1, B+1, \zeta)$ et

$$
\begin{gathered}
J_{\zeta(B+1), \cdots,-\zeta A}^{\theta} \sigma_{A}= \\
J a c_{\zeta(B+1), \cdots,-\zeta A}^{\theta}\left(<\rho||^{\zeta B}, \cdots,\left.\rho\right|^{-\zeta A}>\times \pi(\rho, A-1, B+1, \zeta) \times<\rho||^{\zeta A}, \cdots, \rho^{-\zeta B}>\right)= \\
\operatorname{Jac}_{\zeta(B+1)}^{\theta} \pi(\rho, A-1, B+1, \zeta) .
\end{gathered}
$$

On a aussi, sans l'action de $\theta$ :

$$
J a c_{\zeta(B+1), \cdots,-\zeta A}^{\theta}(\pi(\rho, A, B+1, \zeta) \times \pi(\rho, B, B, \zeta))=\pi(\rho, A-1, B+2, \zeta) \times \pi(\rho, B, B, \zeta) ;
$$

pour avoir l'action de $\theta$, il faut revenir à la définition de $\pi(\rho, A, B+1, \zeta) \times \pi(\rho, B, B, \zeta)$. On veut montrer que l'action de $\theta$ sur le membre de gauche est l'action que nous avons mise sur le membre de droite. Par définition $\theta$ agit sur $\pi(\rho, A, B+1, \zeta) \times \pi(\rho, B, B, \zeta)$ par restriction de l'action naturelle de $\theta$ sur l'induite :

$$
<\rho||^{\zeta(B+1)}, \cdots,\left.\rho\right|^{-\zeta A}>\times \pi(\rho, A-1, B+2, \zeta) \times \pi(\rho, B, B, \zeta) \times<\rho||^{\zeta A}, \cdots, \rho||^{-\zeta(B+1)}>.
$$

Et le résultat est clair.

En regroupant tous les termes ensembles, on obtient :

$$
\begin{gathered}
J_{a c_{\zeta(B+1), \cdots,-\zeta A}^{\theta}}^{\theta} \tilde{\pi}(\rho, A, B, \zeta)=\operatorname{Jac}_{\zeta(B+1)}^{\theta} \pi(\rho, A-1, B+1, \zeta) \oplus_{C \in] B+1, A-1]}(-1)^{A-C} \\
<\rho||^{\zeta B}, \cdots, \rho||^{-\zeta C}>\times J a c_{\zeta(B+3), \cdots, \zeta C}^{\theta} \pi(\rho, A-1, B+3, \zeta) \times<\rho||^{\zeta C}, \cdots, \rho||^{-\zeta B}> \\
\oplus(-1)^{[(A-B+1) / 2]} \pi(\rho, A-1, B+2, \zeta) \times<\rho||^{\zeta B}, \cdots, \rho||^{-\zeta B}>.
\end{gathered}
$$


On remarque que la somme de (6) et (7) n'est autre que $(-1) J a c_{\zeta(B+1)}^{\theta} \tilde{\pi}(\rho, A-1, B+1, \zeta)$. On ne peut pas conclure tout de suite à cause de la différence entre $\pi$ et $\tilde{\pi}$.

Prenons $\tau$ une représentation irréductible de $J a c_{\zeta(B+1)}^{\theta} \tilde{\pi}(\rho, A-1, B+1, \zeta)$ qui reste irréductible après restriction au $G L$ correspondant. On reprend un argument déjà donné; on fixe $\tilde{\tau}$ une représentation irréductible intervenant dans $\tilde{\pi}(\rho, A-1, B+1, \zeta)$ et telle que $J a c_{\zeta(B+1)}^{\theta} \tilde{\tau}$ contienne $\tau$ comme sousquotient. Comme on l'a fait ci-dessus, on démontre que $J a c_{\zeta(B+1)}^{\theta} \tilde{\tau}=\tau$ et que $\tilde{\tau}$ est uniquement déterminé par $\tau$ en particulier est de restriction irréductible au $G L$ correspondant. Par exemple par récurrence on sait que la seule représentation de $\tilde{\pi}(\rho, A-1, B+1, \zeta)$ dont la restriction au GL correspondant est irréductible est $\pi(\rho, A-1, B+1, \zeta)$. On vient donc de montrer que $J a c_{\zeta(B+1)}^{\theta}(\tilde{\pi}(\rho, A-$ $1, B+1, \zeta)-\pi(\rho, A-1, B+1, \zeta))$ ne contient aucune représentation dont la restriction au GL est irréductible.

Revenons à $\tilde{\pi}$ comme dans l'énoncé; on vérifie comme ci-dessus que $J a c_{\zeta(B+1)}^{\theta} \pi$ est irréductible et détermine uniquement $\tilde{\pi}$. Il ne peut donc pas y avoir de simplification dans le module de Jacquet et on vient donc de montrer que $J a c_{\zeta(B+1)}^{\theta} \pi=0$.

On déduit (iii) : on vient de démontrer que pour $\tilde{\pi}$ intervenant dans $\tilde{\pi}(\rho, A, B, \zeta)$ de restriction irréductible à $G L\left(a b d_{\rho}, F\right)$ on a nécessairement $x_{0}=\zeta B$ avec les notations ci-dessus. On a fait le calcul correspondant ci-dessus, il n'y a qu'un terme qui donne ce module de Jacquet, $\pi(\rho, A, B, \zeta)$. Cela termine la preuve de ce cas simple.

\subsection{Résolution dans le groupe de Grothendieck, cas de restriction discrète à la diagonale}

Fixons $\psi$ et $(\rho, a, b) \in \operatorname{Jord}(\psi)$ tel que $\inf (a, b)>1$; on pose encore $A=(a+b) / 2-1, B=|(a-b)| / 2$ et $\zeta$ le signe de $a-b$ si $a \neq b$ et sinon $\zeta$ est un signe quelconque. On note $\psi^{\prime}$ le morphisme qui se déduit de $\psi$ en enlevant le bloc de Jordan $(\rho, a, b)$; on a donc défini $\pi\left(\psi^{\prime}\right)$ comme représentation de $\tilde{G}\left(N_{\psi}-\right.$ $\left.a b d_{\rho}\right)$, ce n'est autre que $\times\left(\rho^{\prime}, a^{\prime}, b^{\prime}\right) \in \operatorname{Jord}(\psi)-(\rho, a, b) S p\left(b^{\prime}, S t\left(a^{\prime}, \rho^{\prime}\right)\right)$. On pose encore $S p(b, S t(a, \rho))=$ $\pi(\rho, A, B, \zeta)$ ce qui permet de définir $\pi(\rho, A, B+2, \zeta)$ et $\pi(\rho, A, B+1, \zeta)$ comme on l'a fait dans 2.1 . On suppose que $\psi \circ \Delta$ est sans multiplicités et on a donc défini $\pi(\psi)$ comme représentation de $\tilde{G}(n)$ dans 1.12

On pose

$$
\begin{gathered}
\tilde{\pi}(\psi)_{\rho, A, B, \zeta}:=\oplus_{C \in] B, A]} \\
(-1)^{A-C}<\rho||^{\zeta B}, \cdots,\left.\rho\right|^{-\zeta C}>\times J a c_{\zeta(B+2), \cdots, \zeta C}^{\theta}\left(\pi\left(\psi^{\prime}\right) \times \pi(\rho, A, B+2, \zeta)\right) \times<\rho||^{\zeta C}, \cdots, \rho||^{-\zeta A}> \\
\oplus(-1)^{[(A-B+1) / 2]} \pi\left(\psi^{\prime}\right) \times \pi(\rho, A, B+1, \zeta) \times<\rho||^{\zeta B}, \cdots, \rho||^{-\zeta B}>.
\end{gathered}
$$

Théorème. L'élément, $\tilde{\pi}(\psi)_{\rho, A, B, \zeta}$ du groupe de Grothendieck associé aux représentations irréductibles de $\tilde{G}$ fait intervenir dans sa décomposition en représentations irréductibles exactement une représentation irréductible de $\tilde{G}$ dont la restriction au GL correspondant est encore irréductible. C'est la représentation $\pi(\psi)$ et elle intervient avec le coefficient +1 . Ou encore, pour tout $g \in G L(n, F) \theta$-semi-simple et régulier,

$$
\operatorname{tr} \tilde{\pi}(\psi)(g, \theta)_{\rho, A, B, \zeta}=\operatorname{tr} \pi(\psi)(g, \theta) .
$$

On traite d'abord le cas où $A=B+1$. On vérifie que par définition $\tilde{\pi}(\psi)_{\rho, A, B, \zeta}$

$$
=<\rho||^{\zeta B}, \cdots \rho||^{-\zeta A}>\times \pi\left(\psi^{\prime}\right) \times<\rho||^{\zeta A}, \cdots, \rho||^{-\zeta B}>\ominus \pi\left(\psi^{\prime}\right) \times \pi(\rho, B+1, B+1, \zeta) \times \pi(\rho, B, B, \zeta) .
$$

L'action de $\theta$ sur le premier terme du membre de droite est l'action prolongeant naturellement celle de $\theta$ sur $\pi\left(\psi^{\prime}\right)$ par contre cette action sur $\pi\left(\psi^{\prime}\right) \times \pi(\rho, B+1, B+1, \zeta) \times \pi(\rho, B, B, \zeta)$ est plus difficile à décrire mais c'est celle que l'on a mise sur $\pi\left(\psi^{\prime \prime}\right)$ où $\psi^{\prime \prime}$ se déduit de $\psi^{\prime}$ en ajoutant les quadruplets $(\rho, B+1, B+1, \zeta)$ et $(\rho, B, B, \zeta)$.

Il est immédiat de vérifier qu'en tant que représentation de $G L(n, F)$ l'induite $<\rho||^{\zeta B},\left.\cdots \rho\right|^{-\zeta A}>$ $\times \pi\left(\psi^{\prime}\right) \times<\rho||^{\zeta A}, \cdots,\left.\rho\right|^{-\zeta B}>$ est de longueur exactement 2 avec comme sous-module $\pi\left(\psi^{\prime}\right) \times$ 
$\pi(\rho, A, B, \zeta)$ (c'est-à-dire $\pi(\psi))$ et comme quotient $\pi\left(\psi^{\prime}\right) \times \pi(\rho, B+1, B+1, \zeta) \times \pi(\rho, B, B, \zeta)$. Le problème est donc de vérifier que cette décomposition donne les "bonnes" actions de $\theta$. Pour le sousmodule, il suffit de suivre les définitions et il n'y a pas de problème. Pour le quotient, si $\psi^{\prime}$ est élémentaire et $B=0$, c'est exactement la définition. Si $B \geq 1 / 2$, c'est la démonstration de 1.14. Reste le cas où $\psi^{\prime}$ n'est pas élémentaire. On fixe alors $\left(\rho^{\prime}, A^{\prime}, B^{\prime}, \zeta^{\prime}\right) \in \operatorname{Jord}\left(\psi^{\prime}\right)$ tel que $A^{\prime}>B^{\prime}$ et on note $\psi^{\prime \prime}$ le morphisme qui se déduit de $\psi^{\prime}$ en remplaçant $\left(\rho^{\prime}, A^{\prime}, B^{\prime}, \zeta^{\prime}\right)$ par $\left(\rho^{\prime}, A^{\prime}-1, B^{\prime}+1, \zeta^{\prime}\right)$ (ou en supprimant ce bloc si $\left.A^{\prime}=B^{\prime}+1\right)$. Toutes les actions de $\theta$ s'obtiennent alors comme on l'a vu en 1.12 en remplaçant dans chaque représentation $\psi^{\prime}$ par $\psi^{\prime \prime}$ d'où une nouvelle représentation, $\sigma$, qu'il faut encore induire

$$
<\rho^{\prime}||^{\zeta^{\prime} B^{\prime}}, \cdots, \rho^{\prime}||^{-\zeta^{\prime} A^{\prime}}>\times \sigma \times<\rho^{\prime}||^{\zeta^{\prime} A^{\prime}}, \cdots, \rho^{\prime}||^{-\zeta^{\prime} B^{\prime}}>,
$$

l'action de $\theta$ étant le prolongement canonique de l'action sur $\sigma$. Il est alors facile d'obtenir le résultat par récurrence. Cela termine la preuve du cas où $A=B+1$.

On suppose donc maintenant que $A>B+1$.

La méthode est très voisine de celle de 2.2 .

Soit $C \in] B, A]$, notons

$$
\sigma_{C}:=<\rho||^{\zeta B}, \cdots, \rho||^{-\zeta C}>\times J a c_{\zeta(B+2), \cdots, \zeta C}^{\theta}\left(\pi\left(\psi^{\prime}\right) \times \pi(\rho, A, B+2, \zeta)\right) \times<\rho||^{\zeta C}, \cdots, \rho||^{-\zeta B}>.
$$

On montre que $\pi\left(\psi^{\prime}\right) \times \pi(\rho, A, B+2, \zeta)$ est l'unique sous-module irréductible de l'induite :

$$
<\rho||^{\zeta(B+2)}, \cdots, \rho||^{\zeta A}>\times \pi\left(\psi^{\prime}\right) \times \pi(\rho, A-1, B+1, \zeta) \times<\rho||^{-\zeta A}, \cdots,\left.\rho\right|^{-\zeta(B+2)}>
$$

l'inclusion étant compatible avec l'action de $\theta$. Cela et le fait que $J a c_{x} \pi\left(\psi^{\prime}\right)=0$ pour tout $x \in[\zeta B, \zeta A]$ entraînent aisément que :

$$
\begin{gathered}
J a c_{\zeta(B+2), \cdots, \zeta C}^{\theta}\left(\pi\left(\psi^{\prime}\right) \times \pi(\rho, A, B+2, \zeta)\right) \hookrightarrow \\
<\rho||^{\zeta(C+1)}, \cdots,\left.\rho\right|^{\zeta A}>\times \pi\left(\psi^{\prime}\right) \times \pi(\rho, A-1, B+1) \times<\left.\rho\right|^{-\zeta A}, \cdots, \rho||^{-\zeta(C+1)}>.
\end{gathered}
$$

On peut même préciser pour la suite que le terme de gauche est l'unique sous-module irréductible du terme de droite, ce qui donne une construction précise de $\sigma_{C}$. Evidemment ici, il est important que $\psi \circ \Delta$ soit sans multiplicités.

On note $\psi_{>}$le morphisme qui se déduit de $\psi$ en enlevant tous les blocs de Jordan $\left(\rho, A^{\prime}, B^{\prime}, \zeta^{\prime}\right)$ tels que $B^{\prime} \leq B$; éventuellement $\psi_{>}$est trivial.

Montrons encore que tout sous-quotient irréductible de $\sigma_{C}$, irréductible en tant que représentation de $\tilde{G}(n)$ et de restriction irréductible à $G L(n, F)$ est de la forme $\pi\left(\psi_{>}\right) \times \sigma^{\prime}$ où $\sigma^{\prime}$ est une représentation convenable d'un groupe linéaire dont le support cuspidal est une collection de représentations $\left.\rho\right|^{x}$ avec $|x| \leq A$. Montrons cette propriété par récurrence sur $\sum_{\left(\rho, A^{\prime}, B^{\prime}, \zeta^{\prime}\right) \in J_{o r d}\left(\psi_{>}\right)}\left(A^{\prime}-B^{\prime}+1\right)$; comme il y a une difficulté pour suivre l'action de $\theta$ alors que la conclusion ne fait pas intervenir cette action, on transforme la propriété en oubliant $\tilde{G}$ et en parlant simplement de représentation irréductible $\theta$ invariante de $G L(N, F)$. Soit $\left(\rho, A_{0}, B_{0}, \zeta_{0}\right)$ l'élément de $\operatorname{Jord}_{\rho}\left(\psi_{>}\right)$avec $A_{0}$ maximum et soit $\tau$ un sous-quotient irréductible de $\sigma_{C}$ pour l'action de $G L(N, F)$; on suppose que $\tau$ est $\theta$-invariant. Suivant ce que l'on a déjà vu, il existe $x_{0}$ un demi-entier tel que $\left|x_{0}\right| \leq A_{0}$ et $\left[x_{0},-\zeta A_{0}\right]$ est un segment et vérifiant :

$$
J a c_{x_{0}, \cdots,-\zeta_{0} A_{0} \tau \neq 0 .}
$$

Un calcul simple montre que $x_{0}=\zeta_{0} B_{0} ;$ si $B_{0}=A_{0}$, on montre tout de suite que $\tau$ s'écrit sous la forme $<\rho||^{\zeta_{0} A_{0}}, \cdots, \rho||^{-\zeta_{0} A_{0}}>\times \tau^{\prime}$ où $\tau^{\prime}$ est irréductible et $\theta$-invariante et vérifie l'analogue de $\tau$ quand on a enlevé le bloc $\left(\rho, A_{0}, B_{0}, \zeta_{0}\right)$. Par contre si $B_{0}<A_{0}$, on montre encore que $J a c_{\zeta_{0} B_{0}, \cdots,-\zeta_{0} A_{0}}^{\theta} \neq 0$. Puis on montre que $\tau$, en tant que représentation de $G L$ est de la forme $<\rho||^{\zeta_{0} B_{0}}, \cdots,\left.\rho\right|^{-\zeta_{0} A_{0}}>$ $\times \tau^{\prime} \times<\rho||^{\zeta_{0} A_{0}}, \cdots, \rho||^{-\zeta_{0} B_{0}}>$, où $\tau^{\prime}$ est un sous-quotient irréductible, $\theta$-invariante de l'analogue de $\sigma_{C}$ obtenu en remplaçant le bloc $\left(\rho, A_{0}, B_{0}, \zeta_{0}\right)$ de $\operatorname{Jord}(\psi) \operatorname{par}\left(\rho, A_{0}-1, B_{0}+1, \zeta_{0}\right)$. Cela permet de conclure. 
Soit donc $\tilde{\sigma}$ un sous-quotient irréductible de $\sigma_{C}$ dont on suppose que la restriction à $G L(n, F)$ est irréductible; on note $\sigma$ cette restriction; on écrit $\sigma$ sous la forme $\pi\left(\psi_{>}\right) \times \sigma^{\prime}$ conformément à ce que l'on vient de voir; on vérifie aisément que $\sigma^{\prime}$ est nécessairement $\theta$ invariante.

Comme dans 2.2, il existe $x_{0}$ un demi-entier tel que $\left|x_{0}\right| \leq A,\left[x_{0},-\zeta A\right]$ soit un segment et tel que $J a c_{x_{0}, \cdots,-\zeta A}^{\theta} \sigma^{\prime} \neq 0$. On en déduit que $J a c_{x_{0}, \cdots,-\zeta A}^{\theta} \sigma \neq 0$ car $\left.\rho\right|^{x} \times \pi\left(\psi_{>}\right)$et $\pi\left(\psi_{>}\right) \times \rho||^{x}$ sont des induites irréductibles pour tout $x$ verifiant $|x| \leq A$. On fixe donc un tel $x_{0}$.

Montrons que nécessairement $x_{0} \in[\zeta B, \zeta A]$ :

ce que l'on obtient immédiatement est que soit $x_{0}$ a la propriété annoncée soit $x_{0}=\zeta^{\prime} B^{\prime}$ pour un bon choix de $\zeta^{\prime}$ et $B^{\prime}$ tel qu'il existe $A^{\prime}$ avec $\left(\rho, A^{\prime}, B^{\prime}, \zeta^{\prime}\right) \in \operatorname{Jord}\left(\psi^{\prime}\right)$. Si cette dernière éventualité se produisait, on remarque que $\pi\left(\psi^{\prime}\right)=\pi\left(\psi_{>}\right) \times \pi\left(\psi_{<}\right)$où $\psi_{<}$correspondant à tous les blocs de Jordan $\left(\rho, A^{\prime \prime}, B^{\prime \prime}, \zeta^{\prime \prime}\right)$ avec $B^{\prime \prime}<B$; mais le support cuspidal de $\pi\left(\psi_{<}\right)$ne contient aucun élément du type $\rho \|^{ \pm x}$ avec $x \in[B, A]$; donc il faudrait nécessairement

$$
\begin{gathered}
J a c_{-\zeta B, \cdots,-\zeta A}\left(<\rho||^{\zeta B}, \cdots, \rho||^{-\zeta C}>\times<\rho||^{\zeta(C+1)}, \cdots, \rho||^{\zeta A}>\right. \\
\left.\times \pi(\rho, A-1, B+1, \zeta) \times<\rho||^{-\zeta A}, \cdots, \rho||^{-\zeta(C+1)}>\times<\rho||^{\zeta C}, \cdots,\left.\rho\right|^{-\zeta B}>\right) \neq 0 .
\end{gathered}
$$

Ce qui est exclu. D'où l'assertion.

On montre la même assertion si $\tilde{\sigma}$ est $\pi\left(\psi^{\prime}\right) \times \pi(\rho, A, B+1, \zeta) \times \pi(\rho, B, B, \zeta)$; dans ce cas, on a même nécessairement $x_{0}=\zeta(B+1)$.

Dans toute la suite de la démonstration, on fixe $\tilde{\sigma}$ une représentation irréductible intervenant dans la décomposition de $\tilde{\pi}(\psi)_{\rho, A, B, \zeta}$ et $x_{0}$ vérifiant la propriété précédente pour $\tilde{\sigma}$. Comme dans 2.2 le point est de démontrer que $x_{0}=\zeta B$. On suppose donc d'abord que $\left.\left.x_{0} \in\right] \zeta B, \zeta A\right]$ et on veut alors montrer simplement que $J a c_{x_{0}}^{\theta} \sigma=0$. Exactement comme dans loc.cit., on vérifie qu'il suffit de montrer que $J a c_{x_{0}}^{\theta} \tilde{\pi}(\psi)_{\rho, A, B, \zeta}=0$. Le cas où $\left.\left.x_{0} \in\right] \zeta(B+1), \zeta A\right]$ est immédiat avec la description précise de $\sigma_{C}$; les termes qui contribuent au calcul est $\sigma_{\left|x_{0}\right|}$ et $\sigma_{\left|x_{0}\right|-1}$ et ils s'éliminent l'un l'autre. Il reste donc à voir le cas plus difficile où $x_{0}=\zeta(B+1)$. On a vu ci-dessus que, pour tout $\left.\left.C \in\right] B, A\right]$ :

$$
\begin{gathered}
\sigma_{C}=<\rho||^{\zeta B}, \cdots, \rho||^{-\zeta C}>\times \\
<<\rho||^{\zeta(C+1)}, \cdots, \rho||^{\zeta A}>\times \pi\left(\psi^{\prime}\right) \times \pi(\rho, A-1, B+1, \zeta) \times<\rho||^{-\zeta A}, \cdots, \rho||^{-\zeta(C+1)}>> \\
\times<\rho||^{\zeta C}, \cdots, \rho||^{-\zeta B}>,
\end{gathered}
$$

où pour $C=A$ le terme du milieu est simplement $\pi\left(\psi^{\prime}\right) \times \pi(\rho, A-1, B+1, \zeta)$. Pour $\left.C \in\right] B+1, A[$, on récrit $\sigma_{C}$. Pour qu'il y ait une valeur de $C$, on peut supposer que $A-1>B+1$, d'où $\pi\left(\psi^{\prime}\right) \times$ $\pi(\rho, A-1, B+1, \zeta)$ est par définition un sous-module (avec action de $\theta$ ) de

$$
<\rho||^{\zeta(B+1)}, \cdots,\left.\rho\right|^{-\zeta(A-1)}>\times \pi\left(\psi^{\prime}\right) \times \pi(\rho, A-2, B+2, \zeta) \times<\rho||^{\zeta(A-1)}, \cdots, \rho||^{-\zeta(B+1)}>.
$$

En revenant à $\sigma_{C}$ pour ces valeurs de $C$,

$$
\begin{gathered}
\sigma_{C} \hookrightarrow<\rho||^{\zeta B}, \cdots, \rho||^{-\zeta C}>\times \\
<\rho||^{\zeta(C+1)}, \cdots, \rho||^{\zeta A}>\times<\rho||^{\zeta(B+1)}, \cdots, \rho||^{-\zeta(A-1)}>\times \\
\pi\left(\psi^{\prime}\right) \times \pi(\rho, A-2, B+2, \zeta) \times \\
<\rho||^{\zeta(A-1)}, \cdots, \rho||^{-\zeta(B+1)}>\times<\rho||^{-\zeta A}, \cdots,\left.\rho\right|^{-\zeta(C+1)}> \\
\times<\rho||^{\zeta C}, \cdots,\left.\rho\right|^{-\zeta B}>.
\end{gathered}
$$

Comme l'action de $\theta$ sur l'induite est l'action qui se déduit naturellement de celle mise sur $\pi\left(\psi^{\prime}\right) \times$ $\pi(\rho, A-2, B+2, \zeta)$, on obtient :

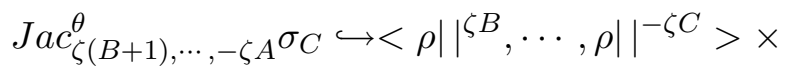




$$
\begin{gathered}
<\rho||^{\zeta(C+1)}, \cdots, \rho||^{\zeta(A-1)}>\times \\
\times\left(\psi^{\prime}\right) \times \pi(\rho, A-2, B+2, \zeta) \times<\rho||^{-\zeta(A-1)}, \cdots, \rho||^{-\zeta C}> \\
\times<\rho||^{\zeta C}, \cdots,\left.\rho\right|^{-\zeta B}>,
\end{gathered}
$$

avec l'action naturelle de $\theta$. Un calcul de module de Jacquet standard montre que cette inclusion se factorise par l'unique sous-module de (3) qui est en fait $J a c_{\zeta(B+3), \cdots, \zeta C}^{\theta}\left(\pi\left(\psi^{\prime}\right) \times \pi(\rho, A-1, B+3, \zeta)\right)$. On a donc montré

$$
\begin{gathered}
J a c_{\zeta(B+1), \cdots,-\zeta A}^{\theta} \sigma_{C}= \\
<\rho||^{\zeta B}, \cdots,\left.\rho\right|^{-\zeta C}> \\
\times J a c_{\zeta(B+3), \cdots, \zeta C}^{\theta}\left(\pi\left(\psi^{\prime}\right) \times \pi(\rho, A-1, B+3, \zeta)\right) \times \\
<\rho||^{\zeta C}, \cdots, \rho||^{-\zeta B}>.
\end{gathered}
$$

Rappelons que ce terme n'apparaît que si $(A-1)>(B+1)$, car $C \in] B+1, A-1]$ et il peut se récrire $J a c_{\zeta(B+1)}^{\theta} \sigma_{C}^{\prime}$ où $\sigma_{C}^{\prime}$ est l'analogue de $\sigma_{C}$ pour $\psi$ remplacé par le morphisme où le bloc $(\rho, A, B, \zeta)$ est remplacé par $(\rho, A-1, B+1, \zeta)$.

On vérifie par un calcul de module de Jacquet standard que pour $C=B+1, J a c_{\zeta(B+1), \cdots,-\zeta A} \sigma_{B+1}=$ 0 et donc a fortiori que $J a c_{\zeta(B+1), \cdots,-\zeta A}^{\theta} \sigma_{B+1}=0$.

On suppose maintenant que $C=A$, et on calcule :

$$
\begin{gathered}
J a c_{\zeta(B+1), \cdots,-\zeta A}^{\theta}\left(<\rho||^{\zeta B}, \cdots, \rho||^{-\zeta A}>\times \pi\left(\psi^{\prime}\right) \times \pi(\rho, A-1, B+1, \zeta) \times<\rho||^{\zeta A}, \cdots, \rho||^{-\zeta(B+1)}>\right)= \\
\operatorname{Jac}_{\zeta(B+1)}^{\theta}\left(\pi\left(\psi^{\prime}\right) \times \pi(\rho, A-1, B+1, \zeta)\right)
\end{gathered}
$$

Il reste donc à calculer

$$
J a c_{\zeta(B+1), \cdots,-\zeta A}^{\theta}\left(\pi\left(\psi^{\prime}\right) \times \pi(\rho, A, B+1, \zeta) \times \pi(\rho, B, B, \zeta)\right) .
$$

Comme on a supposé que $A>B+1$ (le cas d'égalité ayant déjà été traité), on récrit $\pi\left(\psi^{\prime}\right) \times \pi(\rho, A, B+$ $1, \zeta) \times \pi(\rho, B, B, \zeta)$ comme l'unique sous-module irréductible de

$<\rho||^{\zeta(B+1)}, \cdots,\left.\rho\right|^{-\zeta A}>\times \pi\left(\psi^{\prime}\right) \times \pi(\rho, A-1, B+2, \zeta) \times \pi(\rho, B, B, \zeta) \times<\left.\rho||\right|^{\zeta A}, \cdots, \rho||^{-\zeta(B+1)}>$.

Ainsi le $J a c^{\theta}$ cherché est exactement l'induite du milieu, c'est-à-dire $\pi\left(\psi^{\prime}\right) \times \pi(\rho, A-1, B+2, \zeta) \times$ $\pi(\rho, B, B, \zeta)$ avec l'action de $\theta$ mise sur cette induite. On va vérifier que l'on peut récrire le résultat sous la forme :

$$
\begin{gathered}
J a c_{\zeta(B+1), \cdots,-\zeta A}^{\theta}\left(\pi\left(\psi^{\prime}\right) \times \pi(\rho, A, B+1, \zeta) \times \pi(\rho, B, B, \zeta)\right)= \\
J a c_{\zeta(B+1)}^{\theta}\left(\pi\left(\psi^{\prime}\right) \times \pi(\rho, A-1, B+2, \zeta) \times \pi(\rho, B+1, B+1, \zeta)\right)
\end{gathered}
$$

grâce aux définitions on écrit :

$$
\begin{aligned}
& \pi\left(\psi^{\prime}\right) \times \pi(\rho, A, B+1, \zeta) \times \pi(\rho, B, B, \zeta) \hookrightarrow \\
&<\rho||^{\zeta(B+1)}, \cdots, \rho||^{-\zeta A}> \\
& \times \pi(\rho, A-1, B+2, \zeta) \times \pi\left(\psi^{\prime}\right) \times \pi(\rho, B, B, \zeta) \times \\
&<\rho||^{\zeta A}, \cdots, \rho||^{-\zeta(B+1)}>.
\end{aligned}
$$

D'où aisément l'identification du $J a c^{\theta}$ cherché avec l'induite du milieu, c'est-à-dire $\pi(\rho, A-1, B+$ $2, \zeta) \times \pi\left(\psi^{\prime}\right) \times \pi(\rho, B, B, \zeta)$. Mais on a vu en 1.13 que cette induite est aussi l'unique sous-module irréductible de l'induite :

$$
\rho||^{\zeta(B+1)} \times \pi(\rho, A-1, B+2, \zeta) \times \pi\left(\psi^{\prime}\right) \times \pi(\rho, B+1, B+1, \zeta) \times\left.\rho\right|^{-\zeta(B+1)}
$$


inclusion compatible avec les actions de $\theta$. D'où le résultat annoncé.

On remarque que si $A-1=B+1$, ce terme (5) est le même que (4); en tenant compte des signes dans la définition de $\tilde{\pi}(\psi)_{\rho, A, B, \zeta}$, (4) est accompagné de +1 tandis que (5) est accompagné de $(-1)^{[(A-B+1) / 2]}=-1$. Donc si $A=B+2$, on vient de montrer que $\operatorname{Jac}_{\zeta(B+1), \cdots,-\zeta A}^{\theta} \tilde{\pi}(\psi)_{\rho, A, B, \zeta}=0$ et on conclut comme en 2.3 .

Supposons que $A>B+2$. On note $\psi_{-1}$, le morphisme qui se déduit de $\psi$ en remplaçant le bloc de Jordan $(\rho, A, B, \zeta)$ par $(\rho, A-1, B+1, \zeta)$. En remettant les signes, on a donc aussi montré que dans le groupe de Grothendieck de $\tilde{G}\left(N^{\prime}\right)$ pour $N^{\prime}$ convenable :

$$
\begin{gathered}
J a c_{\zeta(B+1), \cdots,-\zeta A}^{\theta} \tilde{\pi}(\psi)_{\rho, A, B, \zeta}= \\
J a c_{\zeta(B+1)}^{\theta} \pi\left(\psi_{-1}\right) \ominus J a c_{\zeta(B+1)}^{\theta} \tilde{\pi}\left(\psi_{-1}\right)_{\rho, A-1, B+1, \zeta} .
\end{gathered}
$$

On conclut encore comme en 2.3. Cela termine la preuve.

\subsection{Le cas général}

Le résultat de 2.3 n'est sans doute pas vrai dans le cas général, c'est-à-dire où l'on ne fait pas l'hypothèse que la restriction à la diagonale est discrète, on rappelle d'ailleurs que pour le moment nous n'avons pas mis d'action de $\theta$ sur $\pi(\psi)$ sans l'hypothèse que $\psi$ est de restriction discrète à la diagonale. Pour ce cas, on réalise $\pi(\psi)$ comme un module de Jacquet convenable. Précisément, pour tout $\rho$; on met un ordre total sur $\operatorname{Jord}_{\rho}(\psi)$ en posant, pour $(\rho, A, B, \zeta),\left(\rho, A^{\prime}, B^{\prime}, \zeta^{\prime}\right) \in \operatorname{Jord}(\psi)$,

$(\rho, A, B, \zeta) \geq\left(\rho, A^{\prime}, B^{\prime}, \zeta^{\prime}\right)$ si

soit $A>A^{\prime}$, soit $A=A^{\prime}$ mais $B>B^{\prime}$ soit $A=A^{\prime}, B=B^{\prime}$ mais $B>0$ et $\zeta=+$.

Il n'y a aucune justification à avoir priviligié $A$ sur $B$, on aurait tout aussi bien pu faire l'inverse. Il n'y a pas non plus de justification pour avoir privilégié le signe + sur le signe - . On étudiera précisément l'influence de nos choix en 5.5

Ayant fait ces choix, on dit qu'un morphisme $\tilde{\psi}$ de $W_{F} \times S L(2, \mathbb{C}) \times S L(2, \mathbb{C})$ dans $G L(N, F)$ avec $N \geq n$ domine $\psi$ s'il est de restriction discrète à la diogonale et s'il existe une bijection de $\operatorname{Jord}(\tilde{\psi})$ sur $\operatorname{Jord}(\psi)$ notée bij vérifiant :

pour tout $\rho$, bij envoie $\operatorname{Jord}_{\rho}(\tilde{\psi})$ sur $\operatorname{Jord}_{\rho}(\psi)$ et bij préserve l'ordre. De plus pour tout $(\rho, \tilde{A}, \tilde{B}, \tilde{\zeta}) \in$ $\operatorname{Jord}_{\rho}(\tilde{\psi})$, en notant $(\rho, A, B, \zeta)$, l'image de cet élément par bij, on a :

$$
\zeta=\tilde{\zeta}, A-B=\tilde{A}-\tilde{B} .
$$

La bijection est clairement uniquement déterminée par le seul fait qu'elle respecte l'ordre.

Comme on part plutôt de $\psi$ que de $\tilde{\psi}$, on notera plutôt $\left(\rho, \tilde{A}_{\rho, A, B, \zeta}, \tilde{B}_{\rho, A, B, \zeta}, \zeta\right)$ l'image inverse de $(\rho, A, B, \zeta) \in \operatorname{Jord}_{\rho}(\psi)$.

On pose alors pour tout $(\rho, A, B, \zeta) \in \operatorname{Jord}(\psi), \mathcal{E}_{\rho, A, B, \zeta}:=\cup_{D \in\left[\tilde{B}_{\rho, A, B, \zeta}, B[\right.}[\zeta D, \zeta(D+A-B)]$, union d'ensembles avec multiplicités. On met sur $\mathcal{E}_{\rho, A, B, \zeta}$ un ordre total en considérant d'abord l'ordre décroissant sur les $D$ puis l'ordre sur le segment $[\zeta D, \zeta(D+A-B)]$.Pour $\rho$ fixé, l'ensemble $\mathcal{E}_{\rho, \tilde{\psi}} \cup_{(A, B, \zeta) \in J_{o r d}(\psi)} \mathcal{E}_{\rho, A, B, \zeta}$ est lui aussi totalement ordonné, en mettant d'abord l'ordre croissant sur les éléments de $\operatorname{Jord}_{\rho}(\psi)$ puis l'ordre sur les ensembles $\mathcal{E}_{\rho, A, B, \zeta}$ que l'on vient de définir. Quand on fait encore l'union sur toues les représentation $\rho$, on obtient un ensemble $\mathcal{E}_{\tilde{\psi}}$ partiellement ordonné. On pose alors, en respectant dans le terme de droite ci-dessous, l'ordre partiel :

$$
\tilde{\pi}(\psi):=J a c_{x \in \mathcal{E}_{\tilde{\psi}}}^{\theta} \pi(\tilde{\psi}) .
$$

Supposons que Cela donne l'action de $\theta$ sur la représentation $\tilde{\pi}(\psi)$.

Théorème, définition : $\tilde{\pi}(\psi)$ est une représentation irréductible de $\tilde{G}(n)$ dont la restriction à $G L(n, F)$ reste irréductible. Précisément cette restriction est $\times_{(\rho, a, b) \in \operatorname{Jord}(\psi)} \operatorname{Sp}(b, S t(a, \rho))$. De plus l'action de $\theta$ sur $\tilde{\pi}(\psi)$ est indépendante du choix fait de $\tilde{\psi}$ dominant $\psi$. On posera donc $\pi(\psi)=\tilde{\pi}(\psi)$. 
On montre ce théorème par récurrence sur $\left|\operatorname{Jord}\left(\psi^{\prime \prime}\right)\right|$ pour tous les morphismes $\psi^{\prime \prime}$ de $W_{F} \times S L(2, \mathbb{C}) \times$ $S L(2, \mathbb{C})$ dans un groupe $G L\left(N^{\prime \prime}, \mathbb{C}\right)$; en effet si ce nombre est réduit à $1, \psi^{\prime \prime} \circ \Delta$ est nécessairement sans multiplicités et le théorème est le corollaire de 1.13 appliqué de proche en proche de $C=\tilde{A}$ jusqu' à $C=A+1$.

En fait il y a 2 assertions dans ce théorème et on va les démontrer successivement. On montre d'abord que la restriction de $\tilde{\pi}(\psi)$ à $G L(n, F)$ est ce que l'on attend à savoir $\times_{(\rho, a, b) \in \operatorname{Jord}(\psi)} S p(b, S t(a, \rho))$.

On fixe $(\rho, A, B, \zeta) \in \operatorname{Jord}(\psi)$, on note $\psi_{<}$le morphisme qui correspond à la représentation de $W_{F} \times S L(2, \mathbb{C}) \times S L(2, \mathbb{C})$ dont les blocs de Jordan sont ceux de

$$
\begin{gathered}
\operatorname{Jord}(\psi)-\operatorname{Jord}_{\rho}(\psi) \cup\left\{\left(\rho, A^{\prime}, B^{\prime}, \zeta^{\prime}\right) \in \operatorname{Jord}_{\rho}(\psi),\left(\rho, A^{\prime}, B^{\prime}, \zeta^{\prime}\right)<(\rho, A, B, \zeta)\right\} \\
\cup\left\{\left(\rho, \tilde{A}^{\prime}, \tilde{B}^{\prime}, \tilde{\zeta}^{\prime}\right) \in \operatorname{Jord}_{\rho}(\tilde{\psi}) ;\left(\rho, \tilde{A}^{\prime}, \tilde{B}^{\prime}, \tilde{\zeta}^{\prime}\right)>\left(\rho, \tilde{A}_{\rho, A, B, \zeta}, \tilde{B}_{\rho, A, B, \zeta}, \zeta\right)\right\} .
\end{gathered}
$$

On admet par récurrence que la restriction de $\tilde{\pi}\left(\psi_{<}\right)$au $G L$ correspondant est ce que l'on attend (on note $\pi\left(\psi_{<}\right)$cette restriction). On va montrer que :

$$
J a c_{x \in \mathcal{E}_{A, B, \zeta}}^{\theta}\left(\pi\left(\psi_{<}\right) \times \pi\left(\rho, \tilde{A}_{\rho, A, B, \zeta}, \tilde{B}_{\rho, A, B, \zeta}, \zeta\right)\right)=\pi\left(\psi_{<}\right) \times \pi(\rho, A, B, \zeta) .
$$

De proche en proche cela donnera la première assertion du théorème.

Pour cela, on montre que pour tout $D \in\left[\tilde{B}_{\rho, A, B, \zeta}, B\right]$ :

$$
\begin{gathered}
J a c_{x \in[\zeta D, \cdots, \zeta(D+A-B)]}^{\theta}\left(\pi\left(\psi_{<}\right) \times \pi(\rho, D+A-B, D, \zeta)\right)= \\
\pi\left(\psi_{<}\right) \times J a c_{x \in[\zeta D, \cdots, \zeta(D+A-B)]}^{\theta} \pi(\rho, D+A-B, D, \zeta)=\pi\left(\psi_{<}\right) \times \pi(\rho, D+A-B-1, D-1, \zeta) .
\end{gathered}
$$

Soit $\mathcal{I}$ un sous-ensemble de $[\zeta D, \zeta(D+A-B)]$ tel que $J a c_{x \in \mathcal{I}} \pi(\rho, D+A-B, D, \zeta) \neq 0$. On se rappelle que $\pi(\rho, D+A-B, D, \zeta)$, comme représentation du $G L$ correspondant, est l'unique sousmodule irréductible de l'induite :

$$
<\rho||^{\zeta D}, \cdots, \rho||^{\zeta(D+A-B)}>\times \pi(\rho, D+A-B-1, D-1, \zeta) \times<\rho||^{-\zeta(D+A-B}, \cdots,\left.\rho\right|^{-\zeta D}>.
$$

Comme pour tout $x \in[\zeta D, \zeta(D+A-B)], J_{a c} \pi(\rho, D+A-B-1, D-1, \zeta)=0$ et qu'il en est de même pour $J a c_{x}<\rho||^{-\zeta(D+A-B)}, \cdots,\left.\rho\right|^{-\zeta D}>$, la non nullité supposée entraîne que $J a c_{x \in \mathcal{I}}<$ $\rho||^{\zeta D}, \cdots, \rho||^{\zeta(D+A-B)}>\neq 0$. En particulier $\mathcal{I}$ est un sous-segment de $[\zeta D, \zeta(D+A-B)]$ commençant par $\zeta D$. Par les formules standard, pour avoir l'égalité de (1) avec le premier membre de (2), la seule chose à démontrer est donc que pour tout $\mathcal{I}^{\prime}$ sous-segment de $[\zeta D, \zeta(D+A-B)]$ contenant l'extrêmité $\zeta(D+A-B), J a c_{x \in \mathcal{I}^{\prime}} \pi\left(\psi_{<}\right)=0$

On décompose encore $\pi\left(\psi_{<}\right)$en $\pi\left(\psi^{\prime}\right) \times \pi\left(\psi_{1}\right) \times \pi\left(\psi_{2}\right)$ où $\operatorname{Jord}\left(\psi^{\prime}\right)=\operatorname{Jord}(\psi)-\operatorname{Jord}_{\rho}(\psi)$, $\operatorname{Jord}\left(\psi_{1}\right)=\left\{\left(\rho, A^{\prime}, B^{\prime}, \zeta^{\prime}\right) \in \operatorname{Jord}(\psi) ;\left(\rho, A^{\prime}, B^{\prime}, \zeta^{\prime}\right)<(\rho, A, B, \zeta)\right\}, \operatorname{Jord}\left(\psi_{2}\right)=\left\{\left(\rho, \tilde{A}^{\prime}, \tilde{B}^{\prime}, \zeta^{\prime}\right) \in\right.$ $\left.\operatorname{Jord}(\tilde{\psi}) ;\left(\rho, \tilde{A}^{\prime}, \tilde{B}^{\prime}, \zeta^{\prime}\right)>\left(\rho, \tilde{A}_{\rho, A, B, \zeta}, \tilde{B}_{\rho, A, B, \zeta}, \zeta\right)\right\}$. Pour $\mathcal{I}^{\prime}$ un sous-ensemble de $[\zeta D, \zeta(D+A-B)]$, on a $J a c_{x \in \mathcal{I}}^{\prime} \pi\left(\psi^{\prime}\right)=0$ pour des raisons de support cuspidal $\operatorname{Jord}_{\rho}\left(\psi^{\prime}\right)=\emptyset$. On a aussi $\operatorname{Jac}_{x \in \mathcal{I}^{\prime}} \pi\left(\psi_{2}\right)=0$ car sinon il faudrait que le premier élément de $\mathcal{I}$, noté $z$, vérifie $J a c_{z} \pi\left(\psi_{2}\right) \neq 0$; il serait alors de la forme $\tilde{\zeta}^{\prime} \tilde{B}^{\prime}$ avec $\left(\rho, \tilde{A}^{\prime}, \tilde{B}^{\prime}, \tilde{\zeta}^{\prime}\right) \in \operatorname{Jord}\left(\psi_{2}\right)$. Or ceci est exclu puisque l'on a certainement $\tilde{B}^{\prime}>\tilde{A}_{\rho, A, B, \zeta}$ par l'hypothèse $\tilde{\psi}$ de restriction discrète à la diagonale, d'où $\tilde{B}^{\prime}>\tilde{A}_{\rho, A, B, \zeta} \geq D+\tilde{A}_{\rho, A, B, \zeta}-\tilde{B}_{\rho, A, B, \zeta}=$ $D+A-B$. Il reste à voir que $J a c_{x \in[\zeta D, \zeta(D+A-B)]} \pi\left(\psi_{1}\right)=0$; or $D+A-B>A$ d'où $D+A-B>A^{\prime}$ pour tout $\left(\rho, A^{\prime}, B^{\prime}, \zeta^{\prime}\right) \in \operatorname{Jord}\left(\psi_{1}\right)$ et $\rho||^{\zeta(D+A-B)}$ n'est pas dans le support cuspidal de $\pi\left(\psi_{1}\right)$. On a donc prouvé l'égalité de (1) avec le premier terme écrit dans (2).

Et l'égalité de (2) résulte de la représentation de $\pi(\rho, D+A-B, D, \zeta)$ donnée en (3). Ceci termine la preuve de $(*)$.

Il reste à montrer que l'action de $\theta$ sur $\tilde{\pi}(\psi)$ est indépendante du choix fait de $\tilde{\psi}$. Fixons 2 choix $\tilde{\psi}_{i}$ pour $i=1,2$ dominant $\psi$; il est clair qu'il existe $\tilde{\psi}$ qui domine les trois morphismes $\tilde{\psi}_{i}$ pour $i=1,2$ et $\psi$ lui-même. On a défini les ensembles ordonnés $\mathcal{E}_{\tilde{\psi}}, \mathcal{E}_{\tilde{\psi}_{i}}$ pour $i=1,2$; ces 2 derniers ensembles sont inclus dans $\mathcal{E}_{\tilde{\psi}}$. Fixons $i$ et posons $\mathcal{F}_{i}:=\mathcal{E}_{\tilde{\psi}}-\mathcal{E}_{\tilde{\psi}_{i}}$. Cet ensemble hérite d'un ordre. Il résulte du corollaire de 1.13 appliqué de nombreuses fois dans l'ordre imposé par l'ordre de $\mathcal{F}_{i}$ que :

$$
J a c_{\mathcal{F}_{i}}^{\theta} \pi(\tilde{\psi})=\pi\left(\tilde{\psi}_{i}\right) .
$$


Le point est donc de démontrer que l'on a :

$$
J a c_{\mathcal{E}_{\tilde{\psi}}}^{\theta}=J a c_{\mathcal{E}_{\tilde{\psi}_{i}}}^{\theta} J a c_{\mathcal{F}_{i}}^{\theta} \pi(\tilde{\psi}),
$$

c'est-à-dire que l'on peut perturber l'ordre de $\mathcal{E}_{\tilde{\psi}}$; on ne perturbe pas n'importe comment (sinon ce serait faux) on utilise simplement le fait que $J a c_{x}^{\theta} J a c_{y}^{\theta}=J a c_{y}^{\theta} J a c_{x}^{\theta}$ si $|y-x|>1$ ce qui ne pose pas de problème ( $\operatorname{cf} 1.4$ ). D'où le théorème.

\subsubsection{Exemples et commentaires}

Dans le paragraphe 2.4, on a fait des choix. Ici, on va donner un exemple qui prouve que le résultat n'est pas indifférent aux choix. On considère l'exemple où $G=G L(4, F)$ et où $\psi$ est trivial sur $W_{F}$ et sur $S L(2, \mathbb{C}) \times S L(2, \mathbb{C})$ est $\left(\operatorname{Rep}_{2} \otimes 1\right) \oplus\left(1 \otimes R e p_{2}\right)$ où $R e p_{2}$ est la représentation irréductible de dimension 2 de $S L(2, \mathbb{C})$. La représentation $\pi(\psi)$ considérée uniquement comme représentation de $G L(4, F)$ est l'induite irréductible $S t(2) \times S p(2)$ où $S t(2)$ est la représentation de Steinberg de $G L(2, F)$ et $S p(2)$ est la représentation triviale de ce groupe.

Pour décrire l'action de $\theta$, on considère $G L(6, F)$ et le morphisme $\tilde{\psi}$ trivial sur $W_{F}$ et valant $R e p_{4} \otimes 1 \oplus 1 \otimes R e p_{2}$ à valeurs dans $G L(6, \mathbb{C})$; ici $R e p_{4}$ est la représentation irréductible de dimension 4. Alors :

$$
\pi(\tilde{\psi}) \hookrightarrow \rho||^{-1 / 2} \times S t(4) \times \rho||^{1 / 2}
$$

avec l'action de $\theta$ qui prolonge naturellement l'action de $\theta$ mise sur $S t(4)$. Et on a par défintion, $\pi(\psi)=J a c_{3 / 2}^{\theta} \pi(\tilde{\psi})$ et cela entraîne que l'action de $\theta$ sur $\pi(\psi)$ est celle qui se déduit naturellement de l'action de $\theta$ mise sur $S t(2)$ en utilisant l'inclusion dans l'induite :

$$
\pi(\psi) \hookrightarrow \rho||^{-1 / 2} \times S t(2) \times \rho||^{1 / 2} .
$$

Pour distinguer, on va noter $\pi_{s t}(\psi)$ cette représentation de $\tilde{G}(4)$. L'autre choix raisonnable aurait été d'inverser les rôles de $S t$ et $S p$ c'est-à-dire de mettre sur $\pi(\psi)$ l'action de $\theta$ qui prolonge naturellement celle mise sur $S p(2)$ en utilisant l'inclusion :

$$
\pi(\psi) \hookrightarrow \rho||^{1 / 2} \times S p(2) \times \rho||^{-1 / 2} .
$$

On note $\pi_{s p}(\psi)$ cette représentation de $\tilde{G}(4)$. Les 2 représentations $\pi_{s t}(\psi)$ et $\pi_{s p}(\psi)$ ne sont pas isomorphes comme nous allons le vérifier.

On considère la série principale :

$$
\sigma:=\rho||^{-1 / 2} \times \rho||^{1 / 2} \times \rho||^{-1 / 2} \times\left.\rho\right|^{1 / 2} .
$$

Cette induite est munie d'une action naturelle de $\theta$ et l'induite (1) en est un sous $\tilde{G}(4)$ module. Ainsi $\pi_{s t}(\psi)$ est un sous- $\tilde{G}(4)$-module. On vérifie par un calcul de module de Jacquet que $\sigma$, en tant que représentation de $G L(4, F)$ est de longueur 4 : elle contient avec multiplicité 1 la représentation $S p(2) \times S p(2)$ et la représentation $S t(2) \times S t(2)$ et avec multiplicité 2 la représentation $S p(2) \times S t(2)$. On vérifie aussi que $\pi_{s p}(\psi)$ est un quotient de $\sigma$; en effet $S p(2)$ muni de l'action de $\theta$ que nous avons mis est quotient de l'induite "du milieu", c'est-à-dire $\rho||^{1 / 2} \times \rho \|^{-1 / 2}$ et $\pi_{s p}(\psi)$ est quotient de l'induite $\left.\rho\right|^{-1 / 2} \times S p(2) \times \rho \|^{1 / 2}$. On note $\pi_{\text {temp }}$ et $\pi_{\text {unip }}$ les représentations de $\tilde{G}(4)$ intervenant dans $\sigma$ dont la restriction à $G L(4, F)$ sont respectivement la représentation tempérée $S t(2) \times S t(2)$ et sa duale $S p(2) \times S p(2)$. Il suffit maintenant de démontrer que pour tout $g \in G L(4, F)$,

$$
\operatorname{tr} \sigma(g, \theta)=\operatorname{tr} \pi_{\text {temp }}(g, \theta)+\operatorname{tr} \pi_{\text {unip }}(g, \theta) .
$$

Pour cela on réalise différemment la représentation de $G L(4, F), S p(2) \times S t(2)$ comme sous-quotients de $\sigma$; on remarque que $S p(2)$ est un sous-module de $\left.\rho\right|^{-1 / 2} \times \rho \|^{1 / 2}$ et que $S t(2)$ en est un quotient; donc $S p(2) \times S t(2)$ est sous-quotient de

$$
\left(\rho||^{-1 / 2} \times \rho||^{1 / 2}\right) \times\left(\rho\left\|^{-1 / 2} \times \rho\right\|^{1 / 2}\right)
$$


Mais $\theta$ ne laisse pas stable cette réalisation et au contraire l'échange avec l'autre réalisation naturelle comme sous-quotient où on considère $S t(2) \times S p(2)$. Donc la trace de $(g, \theta)$ est nulle sur l'espace sous-jacent à la somme de ces 2 réalisations. Ceci prouve notre assertion.

\section{Résolution dans le cas élémentaire}

On fixe encore $\psi$ un morphisme $\theta$-invariante, en supposant $\psi$ élémentaire; par contre, il n'est pas utile qu'il soit de restriction discrète à la diagonale. Il faut faire pour GL(n,F) ce qui a été fait pour les groupes classiques en [14], c'est-à-dire représenter $\pi(\psi)$ à l'aide de la cohomologie d'un complexe exact en tout degré sauf 1 . C'est une réminiscence de [7]. On abrège la notation $G L(?, F)$ en $G L(?)$.

\subsection{Définition du complexe sans action de $\theta$}

Soient $\rho$ une représentation irréductible cuspidale telle que $\theta(\rho)=\rho$ (ou, ce qui est équivalent $\left.\rho \simeq \rho^{*}\right)$ et $d \in \mathbb{N}, d>0$. Quel que soit l'entier $m$, on note $\Pi(\rho, d)$ les représentation irréductibles de $G L(m)$ dont le support cuspidal est formé de représentations de la forme $\left.\rho\right|^{z}$ avec $|z| \leq(d-1) / 2$ et $z-(d-1) / 2 \in \mathbb{Z}$.

Soient $n_{1}, \ldots, n_{s}$ des entiers $\geq 1$, posons :

$$
M=G L\left(n_{1}\right) \times \ldots \times G L\left(n_{s}\right) .
$$

Soit $\sigma=\sigma_{1} \otimes \ldots \otimes \sigma_{s}$ une représentation irréductible de $M$. Notons $\mathcal{S}_{\rho, \leq d}(\sigma)$ l'ensemble des $t \in\{1, \ldots, s\}$ tels que $\sigma_{t} \in \Pi(\rho, \leq d)$. Soit $\pi$ une représentation de $M$. On peut décomposer $\pi$ en somme directe $\pi^{1} \oplus \pi^{2}$ de sorte que, pour tout sous-quotient irréductible $\sigma$ de $\pi^{1}$, resp. $\pi^{2}$, on ait $\left|\mathcal{S}_{\rho, \leq d}(\sigma)\right| \geq s-1$, resp. $\left|\mathcal{S}_{\rho, \leq d}(\sigma)\right| \leq s-2$. On note $\operatorname{proj}_{\rho, \leq d}$ la projection de $\pi$ sur $\pi^{1}$ de noyau $\pi^{2}$. C'est un endomorphisme de $\pi$. On note également $\operatorname{proj}_{\rho, \leq d}$ le foncteur qui, à la représentation $\pi$ de $M$, associe la représentation $\pi^{1}$. Remarquons que cet endomorphisme, resp. ce foncteur, est l'identité si $s=1$.

Soit $n \in \mathbb{N}$. Posons $\Delta=\{1, \ldots, n-1\}$ et fixons un espace vectoriel complexe $E$ de dimension $n-1$, muni d'une base $\left(e_{j}\right)_{j \in \Delta}$. Pour tout entier $j \in\{0, \ldots, n-1\}$, notons $\mathcal{M}_{j}(G L(n))$ l'ensemble des $M \in \mathcal{M}(G L(n))$ tels que la dimension du centre de $M$ soit égale à $j+1$, autrement dit tels que $M$ s'écrive sous la forme (1), avec $s=j+1$ et des entiers $n_{1}, \ldots, n_{s}>0$. Pour $M \in \mathcal{M}_{j}(G L(n))$ écrit sous cette forme, on pose :

$$
\Delta^{M}=\left\{1, \ldots, n_{1}-1\right\} \cup\left\{n_{1}+1, \ldots, n_{1}+n_{2}-1\right\} \cup \ldots \cup\left\{n_{1}+\ldots+n_{j}+1, \ldots, n-1\right\},
$$

et on définit l'élément $e^{M}=e_{n_{1}} \wedge e_{n_{1}+n_{2}} \wedge \ldots \wedge e_{n_{1}+\ldots+n_{j}}$ de $\wedge^{j} E$. Supposons $j \leq n-2$, soient $M \in \mathcal{M}_{j}(G L(n))$ et $M^{\prime} \in \mathcal{M}_{j+1}(G L(n))$, supposons $M^{\prime} \subset M$. Il y a un unique élément $m \in \Delta$ tel que $\Delta^{M}=\Delta^{M^{\prime}} \cup\{m\}$. On a une égalité $e^{M^{\prime}}= \pm e^{M} \wedge e_{m}$ et on note $\xi_{M^{\prime}, M}$ le signe qui y intervient, c'est-à-dire tel que $e^{M^{\prime}}=\xi_{M^{\prime}, M} e^{M} \wedge e_{m}$.

Soit $\pi$ une représentation de $G L(n)$. On va définir un complexe $\mathcal{E}(\pi)=\left(\mathcal{E}_{j}(\pi)\right)_{j \in \mathbb{Z}}$. Pour $j<0$ ou $j \geq n, \mathcal{E}_{j}(\pi)=\{0\}$. Pour $j \in\{0, \ldots, n-1\}$,

$$
\mathcal{E}_{j}(\pi)=\oplus_{M \in \mathcal{M}_{j}(G L(n))} \operatorname{Ind}_{P}^{G L(n)}\left(\operatorname{proj}_{\rho, \leq d}\left(\pi_{P}\right)\right) .
$$

Pour $j<0$ ou $j \geq n-1$, on note $\epsilon_{j}: \mathcal{E}_{j}(\pi) \rightarrow \mathcal{E}_{j+1}(\pi)$ l'application nulle (c'est d'ailleurs l'unique application possible puisqu'au moins un des deux espaces est nul). Soit $j \in\{0, \ldots, n-2\}$. On définit $\epsilon_{j}: \mathcal{E}_{j}(\pi) \rightarrow \mathcal{E}_{j+1}(\pi)$ comme la somme d'applications :

$$
\epsilon_{j, M^{\prime}, M}: \operatorname{Ind}_{P}^{G L(n)}\left(\operatorname{proj}_{\rho, \leq d}\left(\pi_{P}\right)\right) \rightarrow \operatorname{Ind}_{P^{\prime}}^{G L(n)}\left(\operatorname{proj}_{\rho, \leq d}\left(\pi_{P^{\prime}}\right)\right),
$$

la somme étant prise sur les couples $\left(M^{\prime}, M\right) \in \mathcal{M}_{j+1}(G L(n)) \times \mathcal{M}_{j}(G L(n))$. Si $M^{\prime} \not \subset M, \epsilon_{j, M^{\prime}, M}=0$. Supposons $M^{\prime} \subset M$. On a une application canonique :

$$
\pi_{P} \rightarrow \operatorname{Ind} d_{M \cap P^{\prime}}^{M}\left(\pi_{P^{\prime}}\right) .
$$


Considérons l'application composée :

$$
\operatorname{proj}_{\rho, \leq d}\left(\pi_{P}\right) \rightarrow \pi_{P} \rightarrow \operatorname{Ind}_{M \cap P^{\prime}}^{M}\left(\pi_{P^{\prime}}\right) \rightarrow \operatorname{Ind}_{M \cap P^{\prime}}^{M}\left(\operatorname{proj}_{\rho, \leq d}\left(\pi_{P^{\prime}}\right)\right) .
$$

Par fonctorialité, on en déduit une application :

$$
\bar{\epsilon}_{M^{\prime}, M}: \operatorname{Ind} d_{P}^{G L(n)}\left(\operatorname{proj}_{\rho, \leq d}\left(\pi_{P}\right)\right) \rightarrow \operatorname{Ind}_{P}^{G L(n)} \operatorname{Ind}_{M \cap P^{\prime}}^{M}\left(\operatorname{proj}_{\rho, \leq d}\left(\pi_{P^{\prime}}\right)\right)=\operatorname{Ind}_{P^{\prime}}^{G L(n)}\left(\operatorname{proj}_{\rho, \leq d}\left(\pi_{P^{\prime}}\right)\right) .
$$

Alors $\epsilon_{j, M^{\prime}, M}=\xi_{M^{\prime}, M} \bar{\epsilon}_{M^{\prime}, M}$.

Pour que $\mathcal{E}(\pi)$ soit un complexe, on doit vérifier :

(2) pour tout $j \in \mathbb{Z}$, on a l'égalité $\epsilon_{j+1} \circ \epsilon_{j}=0$.

Pour cela, on a besoin de la propriété auxiliaire suivante. Pour deux Lévi $M, M^{\prime} \in \mathcal{M}(G L(n))$ tels que $M^{\prime} \subset M$, notons $\tau_{M^{\prime}, M}: \pi_{P} \rightarrow \pi_{P^{\prime}}$ la projection canonique. Dans $\operatorname{Hom}\left(\pi_{P}, \pi_{P^{\prime}}\right)$, on a l'égalité :

$$
\operatorname{proj}_{\rho, \leq d} \circ \tau_{M^{\prime}, M} \circ \operatorname{proj}_{\rho, \leq d}=\operatorname{proj}_{\rho, \leq d} \circ \tau_{M^{\prime}, M} .
$$

Preuve de (3). Décomposons $\pi_{P}$ en $\pi_{P}^{1} \oplus \pi_{P}^{2}$ comme au début du paragraphe, avec $\pi_{P}^{1}=\operatorname{proj}_{\rho, \leq d}\left(\pi_{P}\right)$. Sur $\pi_{P}^{1}, \operatorname{proj}_{\rho, \leq d}$ est l'identité et l'égalité (2) est claire. Sur $\pi_{P}^{2}, \operatorname{proj}_{\rho, \leq d}$ est nul et on doit montrer que $\operatorname{proj}_{\rho, \leq d} \circ \tau_{M^{\prime}, M}$ l'est aussi. Ecrivons $M$ sous la forme (1) et $M^{\prime}=G L\left(n_{1}^{\prime}\right) \times \ldots \times G L\left(n_{s^{\prime}}^{\prime}\right)$. Il suffit de prouver l'assertion suivante : soient $\sigma=\sigma_{1} \otimes \ldots \otimes \sigma_{s}$ une représentation irréductible de $M$ et $\sigma^{\prime}=\sigma_{1}^{\prime} \otimes \ldots \otimes \sigma_{s^{\prime}}^{\prime}$ une représentation irréductible de $M^{\prime}$; supposons que $\sigma^{\prime}$ intervienne comme sousquotient de $\sigma_{M \cap P^{\prime}}$ et que $\mathcal{S}_{\rho, \leq d}(\sigma)$ ait au plus $s-2$ éléments ; alors $\mathcal{S}_{\rho, \leq d}\left(\sigma^{\prime}\right)$ a au plus $s^{\prime}-2$ éléments. Démontrons cela. Par hypothèse, on peut fixer deux éléments distincts $u, v \in\{1, \ldots, s\}$ tels que $\sigma_{u}$ et $\sigma_{v}$ n'appartiennent pas à $\Pi(\rho, \leq d)$. L'intersection $G L\left(n_{u}\right) \cap M^{\prime}$ est égale à $G L\left(n_{a}^{\prime}\right) \times \ldots \times G L\left(n_{b}^{\prime}\right)$ pour un sous-intervalle $\{a, \ldots, b\}$ de $\left\{1, \ldots, s^{\prime}\right\}$. Puisque $\sigma^{\prime}$ intervient dans $\sigma_{M \cap P^{\prime}}$, le support cuspidal de $\sigma_{u}$ est réunion, en un sens facile à préciser, des supports cuspidaux des $\sigma_{k}^{\prime}$ pour $k \in\{a, \ldots, b\}$. Puisque $\sigma_{u} \notin \Pi(\rho, \leq d)$, il existe $u^{\prime} \in\{a, \ldots, b\}$ tel que $\sigma_{u^{\prime}}^{\prime} \notin \Pi(\rho, \leq d)$, autrement dit $u^{\prime} \notin \mathcal{S}_{\rho, \leq d}\left(\sigma^{\prime}\right)$. En remplaçant $u$ par $v$, on obtient un deuxième indice $v^{\prime}$ tel que $v^{\prime} \notin \mathcal{S}_{\rho, \leq d}\left(\sigma^{\prime}\right)$. Cela prouve $(3)$.

Preuve de (2). On peut supposer $j \in\{0, \ldots, n-3\}$. Alors $\epsilon_{j+1} \circ \epsilon_{j}$ est somme d'applications :

$$
\gamma_{M^{\prime \prime}, M}: \operatorname{Ind} d_{P}^{G L(n)}\left(\operatorname{proj}_{\rho, \leq d}\left(\pi_{P}\right)\right) \rightarrow \operatorname{Ind}_{P^{\prime \prime}}^{G L(n)}\left(\operatorname{proj}_{\rho, \leq d}\left(\pi_{P^{\prime \prime}}\right)\right),
$$

la somme étant prise sur les couples $\left(M^{\prime \prime}, M\right) \in \mathcal{M}_{j+2}(G L(n)) \times \mathcal{M}_{j}(G L(n))$. Ces applications sont définies de la façon suivante. Si $M^{\prime \prime} \not \subset M, \gamma_{M, M^{\prime \prime}}=0$. Supposons $M^{\prime \prime} \subset M$. Notons $m_{1}, m_{2}$ les deux éléments de $\Delta$ tels que $\Delta^{M}=\Delta^{M^{\prime \prime}} \cup\left\{m_{1}, m_{2}\right\}$. Notons $M_{1}^{\prime}$ et $M_{2}^{\prime}$ les éléments de $\mathcal{M}_{j+1}(G L(n))$ tels que $\Delta^{M}=\Delta^{M_{k}^{\prime}} \cup\left\{m_{k}\right\}$ pour $k=1,2$. Alors :

$$
\gamma_{M^{\prime \prime}, M}=\epsilon_{j+1, M^{\prime \prime}, M_{1}^{\prime}} \circ \epsilon_{j, M_{1}^{\prime}, M}+\epsilon_{j+1, M^{\prime \prime}, M_{2}^{\prime}} \circ \epsilon_{j, M_{2}^{\prime}, M} .
$$

On a bien sûr l'égalité $\xi_{M^{\prime \prime}, M_{1}^{\prime}} \xi_{M_{1}^{\prime}, M}+\xi_{M^{\prime \prime}, M_{2}^{\prime}} \xi_{M_{2}^{\prime}, M}=0$. Il suffit alors de vérifier que l'application :

$$
\bar{\epsilon}_{M^{\prime \prime}, M_{k}^{\prime}} \circ \bar{\epsilon}_{M_{k}^{\prime}, M}: \operatorname{Ind} d_{P}^{G L(n)}\left(\operatorname{proj}_{\rho, \leq d}\left(\pi_{P}\right)\right) \rightarrow \operatorname{Ind}_{P^{\prime \prime}}^{G L(n)}\left(\operatorname{proj}_{\rho, \leq d}\left(\pi_{P^{\prime \prime}}\right)\right)
$$

est indépendante de $k \in\{1,2\}$. Soit $f \in \operatorname{Ind}_{P}^{G L(n)}\left(\operatorname{proj}_{\rho, \leq d}\left(\pi_{P}\right)\right)$. Alors $\bar{\epsilon}_{M^{\prime \prime}, M_{k}^{\prime}} \circ \bar{\epsilon}_{M_{k}^{\prime}, M}(f)$ est la fonction qui, en un point $g \in G L(n)$, vaut :

$$
\operatorname{proj}_{\rho, \leq d} \circ \tau_{M^{\prime \prime}, M_{k}^{\prime}} \circ \operatorname{proj}_{\rho, \leq d} \circ \tau_{M_{k}^{\prime}, M}(f(g)) .
$$

D’après (2), ceci est égal à :

$$
\operatorname{proj}_{\rho, \leq d} \circ \tau_{M^{\prime \prime}, M_{k}^{\prime}} \circ \tau_{M_{k}^{\prime}, M}(f(g))
$$

ou encore :

$$
\operatorname{proj}_{\rho, \leq d} \circ \tau_{M^{\prime \prime}, M}(f(g)),
$$

qui est bien indépendant de $k$. 


\subsubsection{Un lemme d'irréductibilité}

Pour prouver l'exactitude du complexe sauf en 1 degré, nous allons utiliser à plusieurs reprise le lemme d'irréductibilité suivant. On fixe $\rho$ une représentation irréductible $\theta$-invariante et $d$ un entier $\geq 1$ comme dans le paragraphe précédent; on reprend aussi la notation $\Pi(\rho, \leq d)$ de ce paragraphe. Soient encore $\pi$ une représentation irréductible de $G L\left(n(\pi)\right.$ (ce qui définit $n(\pi)$ ) et $\psi^{\prime}$ un morphisme élémentaire tel que $\operatorname{Jord}\left(\psi^{\prime}\right)$ soit un ensemble de triplets $\left(\rho_{i}, a_{i}, b_{i}\right)$ pour $i$ dans un ensemble $I$, tel que pour tout $i \in I$ l'une au moins des hypothèses suivantes est vérifiée :

$\rho_{i} \neq \rho, \rho_{i}$ est une représentation cuspidale irréductible autoduale d'un groupe $G L\left(d_{\rho_{i}}\right)$;

$\sup \left(a_{i}, b_{i}\right)-(d-1) / 2 \notin \mathbb{Z}_{\leq 0}$.

On pose $n\left(\psi^{\prime}\right):=\sum_{i \in I} a_{i} b_{i} d_{\rho_{i}}$.

Lemme. Pour tout $\pi \in \Pi(\rho, \leq d)$, les induites $\pi\left(\psi^{\prime}\right) \times \pi$ et $\pi \times \pi\left(\psi^{\prime}\right)$ sont irréductibles et isomorphes.

Rappelons les résultats d'adjonction suivants. Soient $n, n_{1}, n_{2}$ trois entiers $\geq 0$ tels que $n=n_{1}+n_{2}$. Posons $M=G L\left(n_{1}\right) \times G L\left(n_{2}\right), M^{\prime}=G L\left(n_{2}\right) \times G L\left(n_{1}\right)$. Ce sont des Lévi de $G L(n)$. Soient $\sigma_{1}$ et $\sigma_{2}$ des représentations de $G L\left(n_{1}\right)$ et $G L\left(n_{2}\right)$ et $\sigma$ une représentation de $G L(n)$. Alors :

$$
\begin{aligned}
& \operatorname{Hom}_{G L(n)}\left(\sigma, \operatorname{Ind}_{P}^{G L(n)}\left(\sigma_{1} \otimes \sigma_{2}\right)\right)=\operatorname{Hom}_{M}\left(\sigma_{P}, \sigma_{1} \otimes \sigma_{2}\right), \\
& \operatorname{Hom}_{G L(n)}\left(\operatorname{Ind}_{P^{\prime}}^{G L(n)}\left(\sigma_{2} \otimes \sigma_{1}\right), \sigma\right)=\operatorname{Hom}_{M}\left(\sigma_{1} \otimes \sigma_{2}, \sigma_{P}\right) .
\end{aligned}
$$

La première est la réciprocité de Frobenius et la 2e est due à Bernstein.

On va utiliser ces propriétés pour $n_{1}=n\left(\psi^{\prime}\right), n_{2}=n(\pi), n=n_{1}+n_{2}$. Pour tout représentation $\delta$ de $M$, on décompose $\delta$ en $\delta=\delta^{\pi} \oplus \delta^{\neq \pi}$ où tous les sous-quotients irréductibles $\delta_{1} \otimes \delta_{2}$ de $\delta^{\pi}$, resp. $\delta^{\neq \pi}$, vérifient la propriété : $\delta_{2}$ a même support cuspidal que $\pi$, resp. $\delta_{2}$ n'a pas même support cuspidal que $\pi$. Ceci s'applique en particulier quand $\delta$ est le module de Jacquet $\sigma_{P}$ d'une représentation $\sigma$ de $G L(n)$.

Posons $\Sigma=\operatorname{Ind}_{P}^{G L(n)}\left(\pi\left(\psi^{\prime}\right) \otimes \pi\right)$. Soit $\sigma$ un quotient de $\Sigma$. Montrons que :

$$
\sigma_{P}^{\pi} \neq\{0\} .
$$

Notons $\mathbf{x}=\left(x_{1}, \ldots, x_{e}\right)$ une famille de demi-entiers telle que, en posant $\Pi=\rho|\cdot|^{x_{1}} \times \ldots \times \rho|.|^{x_{e}}, \pi$ soit un quotient de $\Pi$. Alors $\sigma$ est un quotient de :

$$
\operatorname{Ind}_{P}^{G L(n)}\left(\pi\left(\psi^{\prime}\right) \otimes \Pi\right)=\left(\times_{i \in I}<\rho_{i}, a_{i}, b_{i}>\right) \times \rho|\cdot|^{x_{1}} \times \ldots \times \rho|\cdot|^{x_{e}}
$$

(l'ensemble $I$ étant muni d'un ordre arbitraire). Cette représentation est isomorphe à :

$$
\operatorname{Ind}_{P^{\prime}}^{G L(n)}\left(\Pi \otimes \pi\left(\psi^{\prime}\right)\right)=\rho|\cdot|^{x_{1}} \times \ldots \times \rho|\cdot|^{x_{e}} \times\left(\times_{i \in I}<\rho_{i}, a_{i}, b_{i}>\right) .
$$

En effet, on passe d'une induite à l'autre en permutant des termes $\left\langle\rho_{i}, a_{i}, b_{i}\right\rangle$ et $\rho|.|^{x_{m}}$ pour $i \in I$ et $m=1, \ldots, e$. D'après l'hypothèse $\pi \in \Pi(\rho, \leq d)$, on a $x_{m} \equiv \frac{d-1}{2} \bmod \mathbb{Z}$ et $\left|x_{m}\right| \leq \frac{d-1}{2}$. L'hypothèse sur $\psi^{\prime}$ et les résultats de Zelevinsky entraînent que les induites $<\rho_{i}, a_{i}, b_{i}>\times \rho|\cdot|^{x_{m}}$ et $\rho|.|^{x_{m}} \times<\rho_{i}, a_{i}, b_{i}>$ sont irréductibles et isomorphes. D'où l'assertion. Alors $\sigma$ est quotient de $\operatorname{Ind}_{P^{\prime}}^{G L(n)}\left(\Pi \otimes \pi\left(\psi^{\prime}\right)\right)$. En appliquant (2), on obtient $\operatorname{Hom}_{M}\left(\pi\left(\psi^{\prime}\right) \otimes \Pi, \sigma_{P}\right) \neq\{0\}$, ce qui implique (3).

Montrons que l'on a :

$$
\Sigma_{P}^{\pi}=\pi\left(\psi^{\prime}\right) \otimes \pi
$$

On utilise la filtration de Bernstein-Zelevinsky. Elle est indexée par $\left[W^{M} \backslash W / W^{M}\right]$. Le quotient correspondant à $w \in\left[W^{M} \backslash W / W^{M}\right]$ est :

$$
\operatorname{Ind}_{M \cap w P w^{-1}}^{M}\left(w\left(\left(\pi\left(\psi^{\prime}\right) \otimes \pi\right)_{M \cap w^{-1} P w}\right)\right) .
$$

Le terme pour $w=1$ donne $\pi\left(\psi^{\prime}\right) \otimes \pi$. Pour un autre $w$, soit $\sigma_{1} \otimes \sigma_{2}$ un sous-quotient irréductible de l'induite ci-dessus. Le support cuspidal de $\sigma_{2}$ contient forcément un terme $\rho_{i}|\cdot|^{\frac{b_{i}-1}{2}}$ ou $\rho_{i}|\cdot|^{\frac{1-a_{i}}{2}}$ pour un 
$i \in I$. D'après les hypothèses sur $\psi^{\prime}$ et $\pi$, un tel terme ne peut pas intervenir dans le support cuspidal de $\pi$. D'où (4).

Soit maintenant $\sigma$ un sous-module irréductible de $\Sigma$ et $\bar{\Sigma}$ le module quotient. D'après (1), $\sigma_{P}^{\pi}$ contient $\pi\left(\psi^{\prime}\right) \otimes \pi$. Par exactitude du foncteur $\delta \mapsto \delta_{P}^{\pi}$ et d'après (4), on a $\bar{\Sigma}_{P}^{\pi}=\{0\}$. Cela contredit (3) sauf si $\bar{\Sigma}=\{0\}$. Donc cette dernière égalité est vérifiée et $\Sigma=\sigma$ est irréductible.

Posons $\Sigma^{\prime}=\operatorname{Ind} d_{P^{\prime}}^{G L(n)}\left(\pi \otimes \pi\left(\psi^{\prime}\right)\right)$. On montre de même que $\Sigma^{\prime}$ est irréductible. D'après (2) et (4), $\operatorname{Hom}_{G L(n)}\left(\Sigma^{\prime}, \Sigma\right) \neq\{0\}$. Par irréductibilité, cela entraîne $\Sigma=\Sigma^{\prime}$.

\subsubsection{Exactitude du complexe}

Soient $\rho, d$ et $n$ comme dans le paragraphe précédent, $\psi$ un morphisme élémentaire; on pose $\operatorname{Jord}(\psi):=\left\{\left(\rho_{i}, a_{i}, b_{i}\right)_{i \in I}\right\}$ pour un ensemble convenable d'indices $I$. On définit les ensembles $J, J_{\leq d}$ par $J:=\left\{i \in I ; \rho_{i}=\rho\right.$ et $\left.\sup \left(a_{i}, b_{i}\right) \equiv d \bmod \mathbb{Z}\right\}$ et $J_{\leq d}:=\left\{i \in J ; \sup \left(a_{i}, b_{i}\right) \leq d\right.$. Posons :

$$
\begin{gathered}
j_{0}(\psi)=\sum_{i \in J_{\leq d}} \sup \left(a_{i}, b_{i}\right), \\
j(\psi)=\left\{\begin{array}{cc}
j_{0}(\psi), & \text { si } I \neq J_{\leq d} \\
j_{0}(\psi)-1, & \text { si } I=J_{\leq d} .
\end{array}\right.
\end{gathered}
$$

Notons $\psi^{\sharp}$ l'élément de $\Psi_{\text {elem,n }}$ formé des $\left(\rho_{i}, a_{i}, b_{i}\right)$ pour $i \notin J_{\leq d}$ et des $\left(\rho_{i}, b_{i}, a_{i}\right)$ pour $i \in J_{\leq d}$.

Proposition. Le complexe $\mathcal{E}(\pi(\psi))$ est exact en degré $<j(\psi)$. Il est nul en degré $>\bar{j}(\psi)$. Le conoyau de $\epsilon_{j(\psi)-1}$ est isomorphe à $\pi\left(\psi^{\sharp}\right)$.

Preuve. Si $I=J_{\leq d}$, la représentation $\pi(\psi)$ appartient à $\Pi(\rho, \leq d)$ et tous les foncteurs $\operatorname{proj}_{\rho, \leq d}$ intervenant dans la définition de $\mathcal{E}(\pi(\psi))$ agissent par l'identité. Le complexe $\mathcal{E}(\pi(\psi))$ est celui qui définit l'involution de Zelevinsky et l'assertion est connue ([]], [22]). On suppose désormais $I \neq J_{\leq d}$. Notons $\psi^{\prime}$ la famille formée des $\left(\rho_{i}, a_{i}, b_{i}\right)$ pour $i \notin J_{\leq d}$. Elle n'est pas vide. Notons $\mathcal{R}$ l'ensemble des représentations de la forme $\pi\left(\psi^{\prime}\right) \times \pi$, où $\pi \in \Pi(\rho, \leq d)$ (il s'agit de représentations de groupes $G L(m)$, où $m$ est quelconque). Soient $j \in\{0, \ldots, n-1\}, M \in \mathcal{M}_{j}(G L(n))$ et $\sigma$ un sous-quotient irréductible de $\operatorname{proj}_{\rho, \leq d}\left(\pi(\psi)_{P}\right)$. Ecrivons :

$$
M=G L\left(n_{1}\right) \times \ldots \times G L\left(n_{j+1}\right), \sigma=\sigma_{1} \otimes \ldots \otimes \sigma_{j+1} .
$$

Montrons qu'il existe $t \in\{1, \ldots, j+1\}$ tel que :

$$
\text { (1) }\left\{\begin{array}{c}
\sigma_{t} \in \mathcal{R} ; \\
\text { pour } r \in\{1, \ldots, j+1\}, \quad r \neq t, \sigma_{r} \in \Pi(\rho, \leq d) .
\end{array}\right.
$$

Par définition de $\operatorname{proj}_{\rho, \leq d}(\pi(\psi))$, on peut en tout cas trouver $t$ tel que la deuxième condition soit satisfaite. Fixons un tel $t$. Rappelons que, par définition, $\pi(\psi)$ est une induite; on écrit $\sigma(\psi)$ la représentation induisante c'est-à-dire $\times_{i \in I} S\left(\rho_{i}, a_{i}, b_{i}\right)$ où pour $i \in I, S\left(\rho_{i}, a_{i}, b_{i}\right)$ est $S t\left(\rho_{i}, a_{i}\right)$ si $b_{i}=1$ et est $S p\left(\rho_{i}, b_{i}\right)$ si $a_{i}=1$. On note $P_{\psi}$ le parbolique (standard) qui permet l'induction.

Filtrons $\pi(\psi)_{P}$ par la filtration de Bernstein-Zelevinsky. Cette filtration est indexée par $\left[W^{M} \backslash W / W^{M_{\psi}}\right]$ et le terme du gradué associé à un élément $w$ de cet ensemble est :

$$
\delta_{w}=\operatorname{Ind} d_{M \cap w P_{\psi} w^{-1}}^{M}\left(w\left(\sigma_{\psi, M_{\psi} \cap w^{-1} P w}\right)\right) .
$$

Soit $w \in\left[W^{M} \backslash W / W^{M_{\psi}}\right]$ tel que $\sigma$ intervienne dans $\delta_{w}$. On a :

(2) pour $i \in I \backslash J_{\leq d}, w$ envoie le bloc de $M_{\psi}$ correspondant à $\left(\rho_{i}, a_{i}, b_{i}\right)$ dans le $t$-ième bloc $G L\left(n_{t}\right)$ de $M$.

En effet, notons $M_{\psi, i}$ le bloc de $M_{\psi}$ en question. Considérons l'ensemble des éléments $r \in\{1, \ldots, j+$ $1\}$ tels que $w M_{\psi, i} w^{-1} \cap G L\left(n_{r}\right) \neq\{1\}$. Notons $r_{1}<\ldots<r_{s}$ ses éléments. Si la conclusion de (2) était fausse, on aurait $r_{1} \neq t$ ou $r_{s} \neq t$. Alors $\sigma_{r_{1}}$, resp. $\sigma_{r_{s}}$, contiendrait dans son support cuspidal une représentation $\rho_{i}|.|^{ \pm \frac{\sup \left(a_{i}, b_{i}\right)-1}{2}}$, ce qui contredirait la propriété $\sigma_{r_{1}} \in \Pi(\rho, \leq d)$, resp. $\sigma_{r_{s}} \in \Pi(\rho, \leq d)$. 
Grâce à (2), $\sigma_{t}$ est sous-quotient d'une induite de la forme $\pi_{1} \times \ldots \times \pi_{u}$ où la famille $\left(\pi_{1}, \ldots, \pi_{u}\right)$ est réunion des $<\rho_{i}, a_{i}, b_{i}>$ pour $i \in I \backslash J_{\leq d}$ et d'une famille d'éléments de $\Pi(\rho, \leq d)$. Le lemme 3.1 .1 entraîne que $\sigma_{t}$ appartient à $\mathcal{R}$, ce qui prouve (1).

De (1) résulte d'abord que, si $\operatorname{proj}_{\rho, \leq d}\left(\pi(\psi)_{P}\right) \neq 0$, il existe $t \in\{1, \ldots, j+1\}$ et des entiers $m_{r} \geq 0$ pour $r \in\{1, \ldots, j+1\}$ de sorte que :

$$
n_{t}=n\left(\psi^{\prime}\right)+m_{t} d_{\rho}, n_{r}=m_{r} d_{\rho} \text { pour } r \neq t .
$$

Cela entraîne $j \leq j(\psi)$. Donc $\mathcal{E}_{j}(\pi(\psi))$ est nul pour $j>j(\psi)$.

Introduisons le Lévi :

$$
\bar{M}=G L\left(n\left(\psi^{\prime}\right)\right) \times G L\left(d_{\rho}\right) \times \ldots \times G L\left(d_{\rho}\right)
$$

de $G L(n)$. Soit $\mathcal{X}$ l'ensemble des familles $\mathbf{x}=\left(x_{k}\right)_{k=1, \ldots, j(\psi)}$ formées de demi-entiers tels que $x_{k} \equiv$ $\frac{d-1}{2} \bmod \mathbb{Z}$ et $\left|x_{k}\right| \leq \frac{d-1}{2}$. Pour toute telle famille $\mathbf{x}$, on définit la représentation de $\bar{M}$ :

$$
\bar{\sigma}_{\mathbf{x}}=\pi\left(\psi^{\prime}\right) \otimes \rho|\cdot|^{x_{1}} \otimes \ldots \otimes \rho|\cdot|^{x_{j(\psi)}} .
$$

Pour tout couple de représentations $\pi_{1}, \sigma_{1}$ d'un Lévi $M_{1}$, avec $\sigma_{1}$ irréductible, notons $\pi_{1}\left[\sigma_{1}\right]$ le facteur direct de $\pi_{1}$ dont tous les sous-quotients ont même support cuspidal que $\sigma_{1}$. Soit $\pi$ une représentation de $G L(n)$ appartenant à $\mathcal{R}$. Il résulte du lemme 3.1.1 et de sa preuve que :

- pour tout $\mathbf{x} \in \mathcal{X}, \pi_{\bar{P}}\left[\bar{\sigma}_{\mathbf{x}}\right]$ est isotypique, de type $\bar{\sigma}_{\mathbf{x}}$;

- il existe $\mathbf{x} \in \mathcal{X}$ tel que $\pi_{\bar{P}}\left[\bar{\sigma}_{\mathbf{x}}\right] \neq 0$.

Soit $j \in\{1, \ldots, j(\psi)\}$. D'après $(1)$ et le lemme 3.1.1, tout sous-quotient irréductible de $\mathcal{E}_{j}(\pi(\psi))$ appartient à $\mathcal{R}$. D'après les propriétés ci-dessus et d'après l'exactitude du foncteur $\pi \mapsto \pi_{\bar{P}}\left[\bar{\sigma}_{\mathbf{x}}\right]$, l'exactitude du complexe $\mathcal{E}(\pi(\psi))$ en degrés $\neq j(\psi)$ résulte de l'assertion suivante :

(3) pour tout $\mathrm{x} \in \mathcal{X}$, la suite :

$$
0 \rightarrow \mathcal{E}_{0}(\pi(\psi))_{\bar{P}}\left[\bar{\sigma}_{\mathbf{x}}\right] \rightarrow \mathcal{E}_{1}(\pi(\psi))_{\bar{P}}\left[\bar{\sigma}_{\mathbf{x}}\right] \rightarrow \ldots \rightarrow \mathcal{E}_{j(\psi)}(\pi(\psi))_{\bar{P}}\left[\bar{\sigma}_{\mathbf{x}}\right]
$$

est exacte.

On reprend maintenant la preuve de [7]. Munissons $W$ de l'ordre de Bruhat, écrivons $W=$ $\left\{w_{1}, \ldots, w_{N}\right\}$ de sorte que, si $w_{m}<w_{m^{\prime}}$, alors $m<m^{\prime}$. Pour $m \in\{1, \ldots, N+1\}$ et $M \in \mathcal{M}(G L(n))$, notons $\Omega_{M, m}$ le plus grand sous-ensemble de $\left\{w_{m}, \ldots, w_{N}\right\}$ qui soit invariant par multiplication à droite par $W^{M}$ et à gauche par $W^{\bar{M}}$. Cet ensemble vérifie les propriétés suivantes :

(4) soient $m, h, h^{\prime} \in\{1, \ldots, N\}$, supposons $w_{h} \in \Omega_{M, m}$ et $w_{h}<w_{h^{\prime}}$. Alors $w_{h^{\prime}} \in \Omega_{M, m}$.

En effet, soient $\ell, \ell^{\prime}$ les entiers tels que $w_{\ell}, w_{\ell^{\prime}}$ soit des éléments de $\left[W^{\bar{M}} \backslash W / W^{M}\right]$ de longueur minimale dans leur double classe et $w_{h} \in W^{\bar{M}} w_{\ell} W^{M}, w_{h^{\prime}} \in W^{\bar{M}} w_{\ell^{\prime}} W^{M}$. On montre aisément que l'inégalité $w_{h}<w_{h^{\prime}}$ entraîne $w_{\ell} \leq w_{\ell^{\prime}}$. Donc $\ell \leq \ell^{\prime}$. Puisque $w_{h} \in \Omega_{M, m}$ et par définition de cet ensemble, on a aussi $w_{\ell} \in \Omega_{M, m}$, donc $\ell \geq m$, puis $\ell^{\prime} \geq m$. Soit $w_{k} \in W^{\bar{M}} w_{\ell^{\prime}} W^{M}$. Alors $w_{k} \geq w_{\ell^{\prime}}$ donc $k \geq \ell^{\prime} \geq m$. Cela montre que $W^{\bar{M}} w_{\ell^{\prime}} W^{M} \subseteq\left\{w_{m}, \ldots, w_{N}\right\}$. Par maximalité de $\Omega_{M, m}$, on a aussi $W^{\bar{M}} w_{\ell^{\prime}} W^{M} \subseteq \Omega_{M, m}$. A fortiori $w_{h^{\prime}} \in \Omega_{M, m}$. Cela prouve (4);

(5) pour $m \leq N$, on a :

$$
\Omega_{M, m}=\left\{\begin{array}{cl}
\Omega_{M, m+1}, & \text { si } w_{m} \notin\left[W^{\bar{M}} \backslash W / W^{M}\right], \\
\left(W^{\bar{M}} w_{m} W^{M}\right) \cup \Omega_{M, m+1}, & \text { si } w_{m} \in\left[W^{\bar{M}} \backslash W / W^{M}\right] .
\end{array}\right.
$$

Il est clair que $\Omega_{M, m} \neq \Omega_{M, m+1}$ si et seulement si $w_{m} \in \Omega_{M, m}$. Si cette condition est vérifiée, on a $W^{\bar{M}} w_{m} W^{M} \subseteq \Omega_{M, m} \subseteq\left\{w_{m}, \ldots, w_{N}\right\}$. Donc $w_{m}$ est minimal pour l'ordre de Bruhat dans sa double classe $W^{\bar{M}} w_{m} W^{M}$ et cela entraîne $w_{m} \in\left[W^{\bar{M}} \backslash W / W^{M}\right]$. Inversement, si $w_{m} \in\left[W^{\bar{M}} \backslash W / W^{M}\right]$, on a pour la même raison $W^{\bar{M}} w_{m} W^{M} \subseteq\left\{w_{m}, \ldots, w_{N}\right\}$, donc $W^{\bar{M}} w_{m} W^{M} \subseteq \Omega_{M, m}$ par maximalité de cet ensemble. Il est clair que $\Omega_{M, m+1} \subseteq \Omega_{M, m} \backslash W^{\bar{M}} w_{m} W^{M}$. Mais ce dernier ensemble est bien inclus dans $\left\{w_{m+1}, \ldots, w_{N}\right\}$ et vérifie les conditions d'invariance requises. La dernière inclusion est donc une égalité ;

(6) si $M^{\prime} \in \mathcal{M}(G L(n))$ est inclus dans $M$, alors $\Omega_{M, m} \subseteq \Omega_{M^{\prime}, m}$. 
C'est évident.

Pour $M \in \mathcal{M}(G L(n))$, on définit une filtration sur $\operatorname{Ind}_{P}^{G L(n)}\left(\operatorname{proj}_{\rho, \leq d}\left(\pi(\psi)_{P}\right)\right)$ indexée par $\{1, \ldots, N+$ 1 \}. Le $m$-ième terme est formé des fonctions à support dans $\bigcup_{w \in \Omega_{M, m}} B w^{-1} B$, ou encore $\bigcup_{w \in \Omega_{M, m}} P w^{-1} \bar{P}$, ces deux ensembles étant égaux. Remarquons que, d'après (4), cet ensemble est ouvert dans $G L(n)$. Chaque terme de la filtration est stable par l'action de $\bar{P}$. De ces filtrations résulte une filtration $\left(\mathcal{E}_{j, m}(\pi(\psi))\right)_{m=1, \ldots, N+1}$ de chaque espace $\mathcal{E}_{j}(\pi(\psi))$. Cette filtration est compatible avec les différentielles $\epsilon_{j}$ d'après (6). Par exactitude du foncteur $\pi \mapsto \pi_{\bar{P}}\left[\bar{\sigma}_{\mathbf{x}}\right]$ (qui est défini pour toute représentation de $\bar{P})$, on a aussi des filtrations $\left(\mathcal{E}_{j, m}(\pi(\psi))_{\bar{P}}\left[\bar{\sigma}_{\mathbf{x}}\right]\right)_{m=1, \ldots, N+1}$ sur les termes $\mathcal{E}_{j}(\pi(\psi))_{\bar{P}}\left[\bar{\sigma}_{\mathbf{x}}\right]$, compatibles aux différentielles. Pour démontrer que la suite (3) est exacte, il suffit de démontrer la même assertion pour la suite des $m$-ièmes gradués, pour tout $m=1, \ldots, N$. Cette suite s'écrit :

$$
\ldots \rightarrow \oplus_{M \in \mathcal{M}_{j-1}(G L(n))} \delta_{M, m}\left[\bar{\sigma}_{\mathbf{x}}\right] \rightarrow \oplus_{M \in \mathcal{M}_{j}(G L(n))} \delta_{M, m}\left[\bar{\sigma}_{\mathbf{x}}\right] \rightarrow \ldots \rightarrow \oplus_{M \in \mathcal{M}_{j(\psi)}(G L(n))} \delta_{M, m}\left[\bar{\sigma}_{\mathbf{x}}\right]
$$

où $\delta_{M, m}$ est le $m$-ième quotient de la filtration de $\left(\operatorname{Ind}_{P}^{G L(n)}\left(\operatorname{proj}_{\rho, \leq d}\left(\pi_{P}\right)\right)\right)_{\bar{P}}$.

On va décrire cette suite. Soient $m \in\{1, \ldots, N\}$ et $M \in \mathcal{M}(G L(n))$. Le $m$-ième gradué de la filtration de $\operatorname{Ind}_{P}^{G L(n)}\left(\operatorname{proj}_{\rho, \leq d}\left(\pi(\psi)_{P}\right)\right)$ est nul, d'après (5), si $w_{m} \notin\left[W^{\bar{M}} \backslash W / W^{M}\right]$. Si $w_{m} \notin\left[W^{\bar{M}} \backslash W\right]$, cette condition est vérifiée pour tout $M$ et la suite (7) est entièrement nulle donc exacte. On suppose désormais $w_{m} \in\left[W^{\bar{M}} \backslash W\right]$. La condition $w_{m} \in\left[W^{\bar{M}} \backslash W / W^{M}\right]$ est alors équivalente à $w_{m} \in\left[W / W^{M}\right]$. Supposons-la vérifiée. D'après (5), on a alors :

$$
\delta_{M, m}=\operatorname{Ind}_{\bar{M} \cap w_{m} P w_{m}^{-1}}^{\bar{M}}\left(w_{m}\left(\left(\operatorname{proj}_{\rho, \leq d}\left(\pi(\psi)_{P}\right)\right)_{M \cap w_{m}^{-1} \bar{P} w_{m}}\right)\right) .
$$

Introduisons quelques notations. Si $w_{m}^{-1} \bar{M} w_{m}$ est un Lévi standard, on le note $\bar{M}_{m}$ et, comme toujours, on note $\bar{P}_{m}$ le sous-groupe parabolique standard qui lui est associé (qui n'a aucune raison d'être égal à $\left.w_{m}^{-1} \bar{P} w_{m}\right)$. On pose :

$$
\bar{\delta}_{m}=w_{m}\left(\pi(\psi)_{\bar{P}_{m}}\left[w_{m}^{-1}\left(\bar{\sigma}_{\mathbf{x}}\right)\right]\right) .
$$

Montrons que :

$$
\left\{\begin{aligned}
\text { si } w_{m}^{-1} \bar{M} w_{m} \not \subset M, & \delta_{M, m}\left[\bar{\sigma}_{\mathbf{x}}\right]=0 \\
\text { si } w_{m}^{-1} \bar{M} w_{m} \subseteq M, & \delta_{M, m}\left[\bar{\sigma}_{\mathbf{x}}\right]=\bar{\delta}_{m}
\end{aligned}\right.
$$

Remarquons que, puisque $w_{m} \in\left[W^{\bar{M}} \backslash W / W^{M}\right]$, l'hypothèse $w_{m}^{-1} \bar{M} w_{m} \subseteq M$ entraîne que $w_{m}^{-1} \bar{M} w_{m}$ est un groupe de Lévi standard de $G L(n)$, ce qui donne un sens à $\bar{\delta}_{m}$.

Ecrivons :

$$
M=G L\left(n_{1}\right) \times \ldots \times G L\left(n_{r}\right), \bar{M}=G_{\bar{n}_{1}} \times \ldots \times G_{\bar{n}_{s}} .
$$

Pour tous $h \in\{1, \ldots, r\}, k \in\{1, \ldots, s\}$, décomposons le sous-groupe $G L\left(n_{h}\right) \cap w_{m}^{-1} \bar{M} w_{m}$ de $M \cap$ $w_{m}^{-1} \bar{M} w_{m}$ en $G L\left(n_{h, 1}\right) \times \ldots \times G L\left(n_{h, s}\right)$ et le sous-groupe $G_{\bar{n}_{k}} \cap w_{m} M w_{m}^{-1}$ de $\bar{M} \cap w_{m} M w_{m}^{-1}$ en $G_{\bar{n}_{k, 1}} \times \ldots \times G_{\bar{n}_{k, r}}$. Les $n_{h, k}$ et $\bar{n}_{k, h}$ sont des entiers $\geq 0$ tels que $n_{h, k}=\bar{n}_{k, h}$. L'action de $w_{m}$ envoie le bloc $G L\left(n_{h, k}\right)$ de $M \cap w_{m}^{-1} \bar{M} w_{m}$ sur le bloc $G_{\bar{n}_{k, h}}$ de $\bar{M} \cap w_{m} M w_{m}^{-1}$. Supposons $\delta_{M, m}\left[\bar{\sigma}_{\mathbf{x}}\right] \neq 0$. On peut fixer un sous-quotient irréductible $\sigma=\sigma_{1} \otimes \ldots \otimes \sigma_{r}$ de $\operatorname{proj}_{\rho, \leq d}\left(\pi(\psi)_{P}\right)$ et une représentation irréductible :

$$
\mu=\mu_{1,1} \otimes \ldots \otimes \mu_{1, s} \otimes \mu_{2,1} \otimes \ldots \otimes \mu_{2, s} \otimes \ldots \otimes \mu_{r, 1} \otimes \ldots \otimes \mu_{r, s}
$$

de $M \cap w_{m}^{-1} \bar{M} w_{m}$ de sorte que les propriétés suivantes soient vérifiées :

- $\mu$ intervient dans $\sigma_{M \cap w_{m}^{-1} \bar{M} w_{m}}$

- une représentation de même support cuspidal que $\bar{\sigma}_{\mathbf{x}}$ intervient dans $\operatorname{Ind}_{\bar{M} \cap w_{m} P w_{m}^{-1}}^{\bar{M}}\left(w_{m}(\mu)\right)$.

Remarquons que la représentation $w_{m}(\mu)$ de $\bar{M} \cap w_{m} M w_{m}^{-1}$ s'écrit :

$$
w_{m}(\mu)=\mu_{1,1} \otimes \ldots \otimes \mu_{r, 1} \otimes \mu_{1,2} \otimes \ldots \otimes \mu_{r, 2} \otimes \ldots \otimes \mu_{1, s} \otimes \ldots \otimes \mu_{r, s} .
$$

A $\sigma$ associons l'entier $t$ vérifiant (1). On a donc $\sigma_{t}=\pi\left(\psi^{\prime}\right) \times \pi$ pour une représentation $\pi \in \Pi(\rho, \leq d)$. Le même raisonnement qui nous a permis de prouver (1) montre que l'une des deux propriétés suivantes est vérifiée : 
- il existe $u \in\{1, \ldots, s\}$ tel que le support cuspidal de $\mu_{t, u}$ contient entièrement celui de $\pi\left(\psi^{\prime}\right)$;

- il existe deux éléments distincts $u, v \in\{1, \ldots, s\}$ tels que $\mu_{t, u}$ et $\mu_{t, v}$ n'appartiennent pas à $\Pi(\rho, \leq d)$.

Rappelons que :

$$
\bar{\sigma}_{\mathbf{x}}=\pi\left(\psi^{\prime}\right) \otimes \rho|\cdot|^{x_{1}} \otimes \ldots \otimes \rho|\cdot|^{x_{j(\psi)}} .
$$

La seconde propriété ci-dessus entraîne que deux facteurs de cette expression n'appartiennent pas à $\Pi(\rho, \leq d)$. Or seul le premier facteur n'appartient pas à cet ensemble. La seconde propriété ci-dessus est donc exclue. Considérons la première. Pour la même raison, on a nécessairement $u=1$ et il y a en fait égalité des supports cuspidaux de $\mu_{t, 1}$ et $\pi\left(\psi^{\prime}\right)$. Donc $n\left(\psi^{\prime}\right)=n_{t, 1}=\bar{n}_{1, t}$. Ces égalités signifient que $w_{m}^{-1}$ envoie le premier bloc de $\bar{M}$ dans le $t$-ième bloc de $M$. Pour le $k$-ième bloc de $\bar{M}$, avec $k \neq 1$, puisque le facteur correspondant de $\bar{\sigma}_{\mathbf{x}}$ est cuspidal, il est clair que la suite $\bar{n}_{k, 1}, \ldots, \bar{n}_{k, r}$ est formée de $r-1$ termes nuls et d'un terme égal à $d_{\rho}$. Cela entraîne encore que $w_{m}^{-1}$ envoie le $k$-ième bloc de $\bar{M}$ dans un bloc de $M$. Cela prouve que $w_{m}^{-1} \bar{M} w_{m} \subseteq M$ et la première assertion de (8) s'ensuit.

Supposons maintenant $w_{m}^{-1} \bar{M} w_{m} \subseteq M$. Alors :

$$
\delta_{M, m}=w_{m}\left(\left(\operatorname{proj}_{\rho, \leq d}\left(\pi(\psi)_{P}\right)_{M \cap w_{m}^{-1} \bar{P} w_{m}}\right)\right.
$$

et :

$$
\delta_{M, m}\left[\bar{\sigma}_{\mathbf{x}}\right]=w_{m}\left(\left(\operatorname{proj}_{\rho, \leq d}\left(\pi(\psi)_{P}\right)_{M \cap w_{m}^{-1} \bar{P} w_{m}}\left[w_{m}^{-1}\left(\bar{\sigma}_{\mathbf{x}}\right)\right]\right) .\right.
$$

Avec la notation introduite dans la preuve de 3.1(3), on a :

$$
\left(\operatorname{proj}_{\rho, \leq d}\left(\pi(\psi)_{P}\right)\right)_{M \cap w_{m}^{-1} \bar{P} w_{m}}\left[w_{m}^{-1}\left(\bar{\sigma}_{\mathbf{x}}\right)\right]=\left(\tau_{\bar{M}_{m}, M} \circ \operatorname{proj}_{\rho, \leq d} \circ \tau_{M, G L(n)}(\pi(\psi))\right)\left[w_{m}^{-1}\left(\bar{\sigma}_{\mathbf{x}}\right)\right] .
$$

Par construction, on a $\operatorname{proj}_{\rho, \leq d}\left(w_{m}^{-1}\left(\bar{\sigma}_{\mathbf{x}}\right)\right)=w_{m}^{-1}\left(\bar{\sigma}_{\mathbf{x}}\right)$ et l'expression ci-dessus est égale à :

$$
\left(\operatorname{proj}_{\rho, \leq d} \circ \tau_{\bar{M}_{m}, M} \circ \operatorname{proj}_{\rho, \leq d} \circ \tau_{M, G L(n)}(\pi(\psi))\right)\left[w_{m}^{-1}\left(\bar{\sigma}_{\mathbf{x}}\right)\right] .
$$

D'après 3.1(3), ceci est égal à :

$$
\left(\operatorname{proj}_{\rho, \leq d} \circ \tau_{\bar{M}_{m}, M} \circ \tau_{M, G L(n)}(\pi(\psi))\right)\left[w_{m}^{-1}\left(\bar{\sigma}_{\mathbf{x}}\right)\right],
$$

ou encore à :

$$
\left(\operatorname{proj}_{\rho, \leq d} \circ \tau_{\bar{M}_{m}, G L(n)}(\pi(\psi))\right)\left[w_{m}^{-1}\left(\bar{\sigma}_{\mathbf{x}}\right)\right] .
$$

Toujours parce que $\operatorname{proj}_{\rho, \leq d}\left(w_{m}^{-1}\left(\bar{\sigma}_{\mathbf{x}}\right)\right)=w_{m}^{-1}\left(\bar{\sigma}_{\mathbf{x}}\right)$, on peut supprimer le $\operatorname{proj}_{\rho, \leq d}$ de cette expression et il ne reste plus que $\left.\tau_{\bar{M}_{m}, G L(n)}(\pi(\psi))\right)\left[w_{m}^{-1}\left(\bar{\sigma}_{\mathbf{x}}\right)\right]$. Alors $\left.\delta_{M, m}\left[\bar{\sigma}_{\mathbf{x}}\right]=w_{m}\left(\tau_{\bar{M}_{m}, G L(n)}(\pi(\psi))\right)\left[w_{m}^{-1}\left(\bar{\sigma}_{\mathbf{x}}\right)\right]\right)$, ce qui démontre la seconde assertion de (8).

Supposons que $w_{m}^{-1} \bar{M} w_{m}$ ne soit pas un Lévi standard. D'après la remarque qui suit (8), on a alors $\delta_{M, m}\left[\bar{\sigma}_{\mathbf{x}}\right]=0$ pour tout $M$. De nouveau, la suite (7) est entièrement nulle, donc exacte. Supposons maintenant que $w_{m}^{-1} \bar{M} w_{m}$ soit un Lévi standard, notons $\Delta_{m} \subseteq \Delta$ son ensemble associé. Identifions $w_{m}$ à une permutation de $\{1, \ldots, n\}$, notons $\Delta_{m}^{\prime} \subseteq \Delta$ l'ensemble des $k \in \Delta$ tels que $w_{m}(k+1)>w_{m}(k)$. Puisque $w_{m} \in\left[W^{\bar{M}} \backslash W\right]$, on a $\Delta_{m} \subseteq \Delta_{m}^{\prime}$. La condition $w_{m}^{-1} \bar{M} w_{m} \subseteq M$ équivaut à $\Delta_{m} \subseteq \Delta^{M}$. La condition $w_{m} \in\left[W / W^{M}\right]$ équivaut à $\Delta^{M} \subseteq \Delta_{m}^{\prime}$. Le résultat de nos calculs s'exprime par l'égalité suivante :

$$
\delta_{M, m}\left[\bar{\sigma}_{\mathbf{x}}\right]=\left\{\begin{array}{cc}
\bar{\delta}_{m}, & \text { si } \Delta_{m} \subseteq \Delta^{M} \subseteq \Delta_{m}^{\prime} \\
0, & \text { sinon }
\end{array}\right.
$$

La suite (7) a donc pour $j$-ième terme :

$$
\oplus_{M \in \mathcal{M}_{j}(G L(n)), \Delta_{m} \subseteq \Delta^{M} \subseteq \Delta_{m}^{\prime}} \bar{\delta}_{m} .
$$

Les différentielles de ce complexe sont évidentes : ce sont les sommes directes d'identités multipliées par les signes $\xi_{M^{\prime}, M}$. Il est bien connu que ce complexe est exact, sauf dans le cas où $\Delta_{m}=\Delta_{m}^{\prime}$, auquel cas il est exact sauf en degré $j=|\Delta|-\left|\Delta_{m}\right|$. Mais $|\Delta|=n\left(\psi^{\prime}\right)+j(\psi) d_{\rho}-1$ et $\left|\Delta_{m}\right|=\left|\Delta^{\bar{M}}\right|=$ 
$n\left(\psi^{\prime}\right)-1+j(\psi)\left(d_{\rho}-1\right)$. Le degré $j$ ci-dessus est donc égal à $j(\psi)$. Cela prouve que le complexe $\mathcal{E}(\pi(\psi))$ est exact en degré $\neq j(\psi)$.

Notons $\pi^{\sharp}$ le conoyau de $\epsilon_{j(\psi)-1}$. C'est une représentation dont on ne sait pas encore qu'elle est irréductible. Toutefois, grâce à (1) et au lemme 3.1.1, tous ses sous-quotients irréductibles appartiennent à $\mathcal{R}$. On peut donc l'écrire $\pi^{\sharp}=\pi\left(\psi^{\prime}\right) \times \pi^{\prime \prime}$, où $\pi^{\prime \prime}$ est une représentation de $G L\left(n-n\left(\psi^{\prime}\right)\right)$ dont tous les sous-quotients appartiennent à $\Pi(\rho, \leq d)$. Pour $\mathbf{x}=\left(x_{k}\right)_{k=1, \ldots, j(\psi)} \in \mathcal{X}$, on a introduit la représentation $\bar{\sigma}_{\mathbf{x}}$ de $\bar{M}$. Introduisons maintenant la représentation :

$$
\sigma_{\mathbf{x}}^{\prime \prime}=\rho|\cdot|^{x_{1}} \otimes \ldots \otimes \rho|\cdot|^{x_{j(\psi)}}
$$

du Lévi $M^{\prime \prime}=G L\left(d_{\rho}\right) \times \ldots \times G L\left(d_{\rho}\right)$ de $G L\left(n-n\left(\psi^{\prime}\right)\right)$. On a $\bar{\sigma}_{\mathbf{x}}=\pi\left(\psi^{\prime}\right) \otimes \sigma_{\mathbf{x}}^{\prime \prime}$. Une preuve analogue à celle du lemme 3.1.1 prouve que :

$$
\pi_{\bar{P}}^{\sharp}\left[\bar{\sigma}_{\mathbf{x}}\right]=\pi\left(\psi^{\prime}\right) \otimes \pi_{P^{\prime \prime}}^{\prime \prime}\left[\sigma_{\mathbf{x}}^{\prime \prime}\right] .
$$

On calcule $\pi_{\bar{P}}^{\sharp}\left[\bar{\sigma}_{\mathbf{x}}\right]$ en reprenant les calculs ci-dessus : il suffit de remplacer le $(j(\psi)+1)$-ième terme du complexe $\mathcal{E}(\pi(\psi))$ par $\pi^{\sharp}$. On obtient que $\pi_{\bar{P}}^{\sharp}\left[\bar{\sigma}_{\mathbf{x}}\right]$ est filtré par une filtration indexée par $\{1, \ldots, N+1\}$. Le $m$-ième terme du gradué associé est nul sauf si $w_{m} \in\left[W^{\bar{M}} \backslash W\right]$ et $w_{m}^{-1} \bar{M} w_{m}$ est un Lévi standard. Si ces conditions sont vérifiées, ce $m$-ième terme du gradué est le dernier conoyau de la suite dont les termes indexés par $j \leq j(\psi)$ sont les termes (9). Comme on l'a vu cette suite est exacte et le conoyau est nul, sauf si $\Delta_{m}=\Delta_{m}^{\prime}$. Dans ce dernier cas, la suite en question est réduite à un seul terme $\bar{\delta}_{m}$ placé en degré $j(\psi)$ et le conoyau est évidemment égal à $\bar{\delta}_{m}$. Or on vérifie qu'il n'y a qu'un élément $m$ vérifiant toutes ces conditions, i.e. $w_{m} \in\left[W^{\bar{M}} \backslash W\right], w_{m}^{-1} \bar{M} w_{m}$ est un Lévi standard, $\Delta_{m}=\Delta_{m}^{\prime}$. Notons $m_{0}$ cet unique $m$. On a $w_{m_{0}}=w_{\max }^{M} w_{\max }$, où $w_{\max }$ est l'élément de longueur maximale de $W$ et $w_{\max }^{\bar{M}}$ l'élément de longueur maximale de $W^{\bar{M}}$. Finalement, on obtient l'égalité :

$$
\pi_{\bar{P}}^{\sharp}\left[\bar{\sigma}_{\mathbf{x}}\right]=\bar{\delta}_{m_{0}} .
$$

Notons $\psi^{\prime \prime}$ la famille formée des $\left(\rho_{i}, a_{i}, b_{i}\right)_{i \in J_{\leq d}}$. Posons $\iota(\mathbf{x})=\left(x_{j(\psi)+1-k}\right)_{k=1, \ldots, j(\psi)}$. On a $\pi(\psi)=$ $\pi\left(\psi^{\prime}\right) \times \pi\left(\psi^{\prime \prime}\right)$ et on prouve une égalité analogue à (10) :

$$
\pi(\psi)_{\bar{P}_{m_{0}}}\left[w_{m_{0}}^{-1}\left(\bar{\sigma}_{\mathbf{x}}\right)\right]=\pi\left(\psi^{\prime \prime}\right)_{P^{\prime \prime}}\left[\sigma_{\iota(\mathbf{x})}^{\prime \prime}\right] \otimes \pi\left(\psi^{\prime}\right)
$$

D'où :

$$
\bar{\delta}_{m_{0}}=w_{m_{0}}\left(\pi\left(\psi^{\prime \prime}\right)_{P^{\prime \prime}}\left[\sigma_{\iota(\mathbf{x})}^{\prime \prime}\right] \otimes \pi\left(\psi^{\prime}\right)\right) .
$$

En utilisant (10) et (11), on en déduit que la multiplicité de $\sigma_{\mathbf{x}}^{\prime \prime}$ dans $\pi_{P^{\prime \prime}}^{\prime \prime}$ est égale à celle de $\sigma_{\iota(\mathbf{x})}^{\prime \prime}$ dans $\pi\left(\psi^{\prime \prime}\right)_{P^{\prime \prime}}$. Notons $\psi^{\prime \prime \sharp}$ la famille formée des $\left(\rho_{i}, b_{i}, a_{i}\right)$ pour $i \in J_{\leq d}$. On sait que $\pi\left(\psi^{\prime \prime \sharp}\right)$ est l'image par l'involution de Zelevinsky de $\pi\left(\psi^{\prime \prime}\right)$. Grâce à [A], on sait que la multiplicité de $\sigma_{\iota(\mathbf{x})}^{\prime \prime}$ dans $\pi\left(\psi^{\prime \prime}\right)_{P^{\prime \prime}}$ est égale à celle de $\sigma_{\mathbf{x}}^{\prime \prime}$ dans $\pi\left(\psi^{\prime \prime}\right)_{P^{\prime \prime}}$. Finalement, on a deux représentations $\pi^{\prime \prime}$ et $\pi\left(\psi^{\prime \prime \sharp}\right)$ de $G L\left(n-n\left(\psi^{\prime}\right)\right)$ qui vérifient les propriétés suivantes :

- $\pi\left(\psi^{\prime \prime \sharp}\right)$ est irréductible et appartient à $\Pi(\rho, \leq d)$;

- tout sous-quotient irréductible de $\pi^{\prime \prime}$ appartient à $\Pi(\rho, \leq d)$;

- pour tout $\mathbf{x} \in \mathcal{X}$, les multiplicités de $\sigma_{\mathbf{x}}^{\prime \prime}$ dans $\pi\left(\psi^{\prime \prime \sharp}\right)_{P^{\prime \prime}}$ et dans $\pi_{P^{\prime \prime}}^{\prime \prime}$ sont égales.

Il en résulte que $\pi^{\prime \prime}=\pi\left(\psi^{\prime \prime \sharp}\right)$. En effet, munissons $\mathcal{X}$ de l'ordre lexicographique. Pour toute représentation irréductible $\mu$ de $G L\left(n-n\left(\psi^{\prime}\right)\right)$, appartenant à $\Pi(\rho, \leq d)$, notons $\mathbf{x}(\mu)$ le plus grand élément de l'ensemble des $\mathbf{x} \in \mathcal{X}$ tels que $\mu_{P^{\prime \prime}}\left[\sigma_{\mathbf{x}}^{\prime \prime}\right] \neq 0$. D'après $[\mathrm{Z}]$ proposition 6.9 , l'application $\mu \mapsto \mathbf{x}(\mu)$ est injective. Considérons l'élément $\mathbf{x}^{\sharp}=\mathbf{x}\left(\pi\left(\psi^{\prime \prime}\right)\right)$. On a $\pi\left(\psi^{\prime \prime \sharp}\right)_{P^{\prime \prime}}\left[\sigma_{\mathbf{x}^{\sharp}}^{\prime \prime}\right] \neq 0$, donc aussi $\pi_{P^{\prime \prime}}^{\prime \prime}\left[\sigma_{\mathbf{x}^{\sharp}}^{\prime \prime}\right] \neq 0$. Fixons un sous-quotient irréductible $\pi_{0}^{\prime \prime}$ de $\pi^{\prime \prime}$ tel que $\pi_{0, P^{\prime \prime}}^{\prime \prime}\left[\sigma_{\mathbf{x}^{\sharp}}^{\prime \prime}\right] \neq 0$. Alors $\mathbf{x}^{\sharp}$ est encore maximal parmi les $\mathbf{x} \in \mathcal{X}$ tels que $\pi_{0, P^{\prime \prime}}^{\prime \prime}\left[\sigma_{\mathbf{x}}^{\prime \prime}\right] \neq 0$. Donc $\mathbf{x}\left(\pi_{0}^{\prime \prime}\right)=\mathbf{x}^{\sharp}$. D'après le résultat de Zelevinsky que l'on vient de citer, $\pi_{0}^{\prime \prime}=\pi\left(\psi^{\prime \prime} \sharp\right)$. Mais alors on a aussi $\pi^{\prime \prime}=\pi_{0}^{\prime \prime}$ car les propriétés ci-dessus impliquent qu'il n'y a plus de place pour d'autres sous-quotients. Cela démontre notre assertion.

On a maintenant :

$$
\pi\left(\psi^{\sharp}\right)=\pi\left(\psi^{\prime}\right) \times \pi^{\prime \prime}=\pi\left(\psi^{\prime}\right) \times \pi\left(\psi^{\prime \prime}\right)=\pi\left(\psi^{\sharp}\right),
$$

ce qui achève la preuve. 


\subsection{Action de $\theta$}

Les données et les notations sont les mêmes que dans 3.1.2. Prolongeons $\pi(\psi)$ en une représentation $\pi(\psi)^{+}$de $\tilde{G}(n)$. Alors on peut munir chaque terme $\mathcal{E}_{j}(\pi(\psi))$ du complexe $\mathcal{E}(\pi(\psi))$ d'un prolongement à $\tilde{G}(n)$ que l'on note $\mathcal{E}_{j}\left(\pi(\psi)^{+}\right)$. Il se définit de la façon suivante. Soit $M \in \mathcal{M}_{j}(G L(n))$. De $\pi(\psi)^{+}(\theta)$ se déduit naturellement un homomorphisme de $\pi(\psi)_{P}$ dans $\pi(\psi)_{\theta(P)}$, qui se restreint évidemment en un homomorphisme de $\operatorname{proj}_{\rho, \leq d}\left(\pi(\psi)_{P}\right)$ dans $\operatorname{proj}_{\rho, \leq d}\left(\pi(\psi)_{\theta(P)}\right)$. On induit cet homomorphisme en un homomorphisme :

$$
\operatorname{Ind}_{P}^{G L(n)}\left(\operatorname{proj}_{\rho, \leq d}\left(\pi(\psi)_{P}\right)\right) \rightarrow \operatorname{Ind}_{\theta(P)}^{G L(n)}\left(\operatorname{proj}_{\rho, \leq d}\left(\pi(\psi)_{\theta(P)}\right)\right) .
$$

L'action de $\theta$ sur $\mathcal{E}_{j}(\pi(\psi))$ est la somme sur les $M \in \mathcal{M}_{j}(G L(n))$ de ces homomorphismes, multipliée $\operatorname{par}(-1)^{\left[\frac{j}{2}\right]}$. On vérifie sans peine que les différentielles $\epsilon_{j}$ sont équivariantes pour cette action de $\theta$.

Remarque. C'est pour assurer cette compatibilité que l'on a introduit la multiplication par $(-1)^{\left[\frac{j}{2}\right]}$. En effet, si on omet ces signes, on a équivariance non pas des différentielles $\epsilon_{j}$, mais des applications $\bar{\epsilon}_{j}$ définies comme $\epsilon_{j}$, mais en oubliant les signes $\xi_{M^{\prime}, M}$. Avec notre définition, on obtient la bonne équivariance en vertu de l'égalité suivante : soient $M \in \mathcal{M}_{j}(G L(n)), M^{\prime} \in \mathcal{M}_{j+1}(G L(n))$ deux Lévi tels que $M^{\prime} \subset M$; alors $(-1)^{\left[\frac{j}{2}\right]} \xi_{M^{\prime}, M}=(-1)^{\left[\frac{j+1}{2}\right]} \xi_{\theta\left(M^{\prime}\right), \theta(M)}$.

Le conoyau de $\epsilon_{j(\psi)-1}$ se retrouve naturellement muni d'un prolongement à $\tilde{G}(n)$. Grâce à la proposition 15, on obtient ainsi un prolongement $\pi\left(\psi^{\sharp}\right)^{+}$de $\pi\left(\psi^{\sharp}\right)$.

on pose $\beta(\psi, \rho, \leq d)=\left\{\begin{array}{l}(-1)^{\left|J_{\leq d}\right|\left(\left|J_{\leq d}\right|-1\right) / 2} \prod_{i \in J_{\leq d}}(-1)^{\left(a_{i} b_{i}-1\right) / 2}, \text { si } d \text { est impair } \\ \prod_{i \in J_{\leq d}}(-1)^{\left(a_{i} b_{i}-1\right) / 2}, \text { si } d \text { est pair }\end{array}\right.$.

On remarque que ce signe ne dépend que de $\psi \circ \Delta$, on le note donc $\beta(\psi \circ \Delta, \rho, \leq d)$. On vérifie encore aisément que $\beta(\psi \circ \Delta, \rho, \leq d)$ vaut $(-1)^{[j(\psi) / 2]}$, où $j(\psi)$ a été défini au début de 3.1.2.

Lemme. L'action de $\theta$ sur $\pi\left(\psi^{\sharp}\right)$ est celle que nous y avons mise si et seulement si $\beta(\psi, \rho, \leq d)=+1$.

Comme $\psi$ est élémentaire pour tout $(\rho, a, b) \in \operatorname{Jord}(\psi)$, le quadruplet correspondant $(\rho, A, B, \zeta)$ vérifie $A=B$. On supprimera donc le $A$ de la notation. On va faire la démonstration dans le cas où $B$ est demi-entier pour tout $(\rho, B, \zeta) \in \operatorname{Jord}(\psi)$. Le cas opposé donne une écriture plus compliquée mais n'introduit pas de signe. On note $\psi_{>d}$ le morphisme qui se déduit de $\psi$ en enlevant tous les blocs $(\rho, B, \zeta)$ avec $B \leq(d-1) / 2$.

Par définition $\pi(\psi)$ est un sous-module irréductible de l'induite :

$$
\begin{aligned}
\pi(\psi) \hookrightarrow & \left(\times_{(\rho, B, \zeta) \in \operatorname{Jord}(\psi) ; B \leq(d-1) / 2}<\rho||^{\zeta B}, \cdots, \rho||^{\zeta 1 / 2}>\right) \times \pi\left(\psi_{>d}\right) \\
& \times\left(\times_{(\rho, B, \zeta) \in \operatorname{Jord}(\psi) ; B \leq(d-1) / 2}<\rho||^{-\zeta 1 / 2}, \cdots,\left.\rho\right|^{-\zeta B}>\right)
\end{aligned}
$$

le produit se fait en prenant les $B$ dans l'ordre croissant pour le terme de gauche et décroissant pour le terme de droite. On vient de démontrer que l'image de $\pi(\psi)$ par $i n v_{\leq d}$ est un sous-module irréductible de l'induite :

$$
\begin{aligned}
\pi\left(\psi^{\sharp}\right) \hookrightarrow & \left(\times_{(\rho, B, \zeta) \in \operatorname{Jord}(\psi) ; B \leq(d-1) / 2}<\rho||^{-\zeta B}, \cdots,\left.\rho\right|^{-\zeta 1 / 2}>\right) \times \pi\left(\psi_{>d}\right) \\
& \left(\times_{(\rho, B, \zeta) \in \operatorname{Jord}(\psi) ; B \leq(d-1) / 2} \times<\left.\rho\right|^{\zeta 1 / 2}, \cdots,\left.\rho\right|^{\zeta B}>\right) .
\end{aligned}
$$

L'action $\theta(\psi)$ mise sur $\pi(\psi)$ est la restriction à $\pi(\psi)$ du prolongement canonique de $\theta\left(\psi_{>d}\right)$ (cf. (1)) et on veut montrer que l'action de $\theta$ que l'on obtient par la résolution du complexe a la même propriété en utilisant (2). Pour cela on note $\pi\left(\psi^{\sharp}\right)^{+}$la représentation munie de l'action $\theta\left(\psi^{\sharp}\right)$ et $\pi\left(\psi^{\sharp}\right)^{-}$la représentation munie de l'action $-\theta\left(\psi^{\sharp}\right)$. On pose

$$
\sigma:=\left(\left.\otimes_{(\rho, B, \zeta) \in \operatorname{Jord}(\psi) ; B \leq(d-1) / 2} \otimes_{k \in[-\zeta B,-\zeta 1 / 2]} \rho\right|^{-k}\right) \otimes \pi\left(\psi_{>d}\right)
$$




$$
\left(\otimes_{(\rho, B, \zeta) \in \operatorname{Jord}(\psi) ; B \leq(d-1) / 2} \otimes_{k \in[\zeta 1 / 2, \zeta B]} \rho||^{k}\right) .
$$

On note $M_{0}$ le Levi sur lequel vit $\sigma$ et $P_{0}$ le parabolique standard de Levi $M_{0}$. On note encore $\sigma^{+}$la représentation $\sigma$ munie de l'action de $\theta$ qui échange la partie gauche avec la partie droite et qui agit par $\theta\left(\psi_{>d}\right)$ sur la représentation du milieu. Et on note $\sigma^{-}$la même représentation où on remplace $\theta\left(\psi_{>d}\right)$ par son opposé. On vérifie aisément que $\sigma^{+}$intervient dans le module de Jacquet de $\pi\left(\psi^{\sharp}\right)_{P_{0}}^{+}$ alors que $\sigma^{-}$n'y intervient pas. Pour $\pi\left(\psi^{\sharp}\right)^{-}$, les rôles sont inversés. Pour $\tau$ une représentation, avec action de $\theta$, du Levi sur lequel vit $\sigma$, on note $\tilde{m}(\tau)$ la multiplicité de $\sigma^{+}$moins la multiplicité de $\sigma^{-}$. L'intérêt d'une telle multiplicité est qu'elle vaut 0 pour toute représentation de la forme $\tau \oplus \tau^{\prime}$ si $\theta$ échange les 2 facteurs. En effet la trace de $\theta$ y vaut 0 et donc la multiplicité avec laquelle $\sigma^{+}$intervient est la même que celle avec laquelle $\sigma^{-}$intervient.

Soit $P$ un sous-groupe parabolique standard de Levi $M$; pour toute représentation $\delta$ de $M$, on utilise la description du semi-simplifié de $\left(\operatorname{Ind} d_{P}^{G L(n)} \delta\right)_{P_{0}}$ comme la somme des termes indexés par les éléments du groupe de Weyl, $w$, de longueur minimale à gauche modulo le groupe de Weyl de $M_{0}$ et à droite modulo le groupe de Weyl de $M, \operatorname{Ind}_{M_{0} \cap w P w^{-1}}^{M_{0}} w\left(\delta_{M \cap w^{-1} P_{0} w}\right)$. On note $I_{P, w}$ ce terme pour $\delta$ $=\operatorname{proj}_{\rho, \leq d} \pi(\psi)_{P}$. Montrons que, la première somme ci-dessous porte sur les paraboliques standard de rang fixé :

$$
\tilde{m}\left(\sum_{P}\left(\operatorname{Ind}_{P}^{G L(n)}\left(\operatorname{proj}_{\rho, \leq d} \pi(\psi)_{P}\right)_{P_{0}}\right)=\sum_{P ; \theta(M)=M} \tilde{m}\left(\operatorname{Ind}_{P}^{G L(n)}\left(\operatorname{proj}_{\rho, \leq d} \pi(\psi)_{P}\right)_{P_{0}}\right) .\right.
$$

En effet, on regroupe dans la première somme le parabolique de Levi $M$ avec celui de Levi $\theta(M)$; si ces 2 Levi sont différents, $\theta$ échange les 2 termes $\sigma$ isotypiques correspondants et agit donc par une représentation de trace 0 . On fixe donc $P$ de Levi $\theta$-invariante; de la même façon, on montre que l'on peut limiter la somme aux éléments $w$ qui sont $\theta$-invariantes. Soient $M$ et $w \theta$-invariantes; montrons que $\tilde{m}\left(I_{P, w}\right)=0$ si $w^{-1} M_{0} w \not \subset M$. En effet, on écrit

$$
M=G L\left(n_{1}\right) \times \cdots \times G L\left(n_{r}\right) \times G L\left(n_{0}\right) \times G L\left(n_{r}\right) \times \cdots \times G L\left(n_{1}\right),
$$

avec des $n_{i}>0$ pour $i \in[1, r]$ et $n_{0} \geq 0$. On a défini $\operatorname{proj}_{\rho, \leq d}$ pour les représentations de $M$; on note $\operatorname{proj}_{\rho, \leq d}^{\theta}$ la somme des sous-modules irréductibles de $\operatorname{proj}_{\rho, \leq d}$ de la forme $\otimes_{i \in[1, r]} \sigma_{i} \otimes \sigma_{0} \otimes_{i \in[r, 1]}$ ${ }^{\theta} \sigma_{i}$. Par les arguments ci-dessus, on voit que l'on peut remplacer dans les calculs $\operatorname{proj}_{\rho, \leq d} \pi(\psi)_{P}$ par $\operatorname{proj}_{\rho, \leq d}^{\theta} \pi(\psi)_{P}$. Et comme par définition une au plus des représentations $\delta^{\prime}$ dans l'union des 3 ensembles

$$
\left\{\sigma_{i} ; i \in[1, r]\right\} \cup\left\{{ }^{\theta} \sigma_{i} ; i \in[1, r]\right\} \cup\left\{\sigma_{0}\right\}
$$

pourrait contenir dans son support cuspidal $\rho||^{y}$ avec $|y|>(d-1) / 2$ ou $\left.y-(d-1) / 2\right) \notin \mathbb{Z}$, ce n'est pas le cas des représentations $\sigma_{i}$ pour $i \in[1, r]$. Le fait que $w$ est de longueur minimale dans sa classe modulo $M$ et la forme de $M_{0}$ entraîne alors l'assertion et entraîne même que $w^{-1} M_{0} w=M_{0}$.

Soit donc $P, M, w$ comme ci-dessus, tels que $M$ et $w$ soient $\theta$-invariantes et tels que $w^{-1} M_{0} w=M_{0}$. Alors $\tilde{m} I_{P, w}=\tilde{m}\left(w\left(\pi(\psi)_{P_{0}}\right)\right)$.

On note $W\left(M_{0}\right)$ l'ensemble des éléments du groupe de Weyl $w$ tel que $w M_{0} w^{-1}=M_{0}$ et $W_{M_{0}}$ le groupe de Weyl de $M_{0}$. Notons ici $\pi_{\sharp}$ la représentation $\pi\left(\psi^{\sharp}\right)$ munie de l'action de $\theta$ qui lui vient du complexe. Pour tout entier $j$, notons encore $\mathcal{M}_{j}^{\theta}$ l'ensemble des Levi standard de corang $j$ qui sont $\theta$ invariants et qui contiennent $M_{0}$. On a alors montré que :

$$
\tilde{m}\left(\left(\pi_{\sharp}\right)_{P_{0}}\right)=\sum_{j}(-1)^{j+[j / 2]-j(\psi)} \sum_{M \in \mathcal{M}_{j}^{\theta}} \sum_{w \in W_{M_{0}} \backslash W\left(M_{0}\right) ; \theta(w)=w} \tilde{m}\left(w\left(\pi(\psi)_{P_{0}}\right)\right) .
$$

On remarque que $(-1)^{j+[j / 2]}=(-1)^{-j+[j / 2]}=(-1)^{[(j+1) / 2]}$. On peut inverser l'ordre des sommations, c'est-à-dire fixer $w$ et sommer sur les Levi standard $M, \theta$-invariantes tels que $w$ soit de longueur minimale dans sa classe à droite modulo $M$. Pour $w$ fixé, on pose $\Delta^{w}$ l'ensemble des racines simples positives hors de $M_{0}$ dont les images par $w$ soient encore positives. Cet ensemble est $-\theta$-invariante 
et on note $\Delta_{\theta}^{w}$ les classes de conjugaison pour cette action $-\theta$. Un Levi standard $M, \theta$-invariante tel que $w$ soit minimal dans sa classe à droite modulo $M$ est précisément défini pour un sous-ensemble de $\Delta_{\theta}^{w}$. Ainsi, si $\Delta_{\theta}^{w}$ est non vide, la somme alternée est nulle. Il n'y a qu'un seul élément $w$ pour lequel $\Delta^{w}$ est vide, celui qui envoie toutes les racines positives hors de $M_{0}$ sur des racines négatives; notons le $w_{0}$. On a

$$
\begin{aligned}
w_{0} \sigma= & \left(\otimes_{(\rho, B, \zeta) \in \operatorname{Jord}(\psi) ; B \leq(d-1) / 2} \otimes_{k \in[\zeta B, \zeta 1 / 2]} \rho||^{-k}\right) \otimes \pi\left(\psi_{>d}\right) \\
& \left(\otimes_{(\rho, B, \zeta) \in \operatorname{Jord}(\psi) ; B \leq(d-1) / 2} \otimes_{k \in[-\zeta 1 / 2,-\zeta B]} \rho||^{k}\right) .
\end{aligned}
$$

Si $\psi$ est de restriction discrète à la diagonale (cas que nous utiliserons), la multiplicité de cette représentation dans $\pi(\psi)_{P_{0}}$ est précisément 1 avec comme action de $\theta$ l'action qui se déduit naturellement de $\theta\left(\psi_{>d}\right)$. Avec (3), cela donne :

$$
\tilde{m}\left(\left(\pi_{\sharp}\right)_{P_{0}}\right)=(-1)^{j(\psi)+[j(\psi) / 2]-j(\psi)}=(-1)^{[j(\psi) / 2]} .
$$

C'est le résultat annoncé.

\section{Transfert, contexte et propriétés générales}

\subsection{Les groupes}

Soit $n \in \mathbb{N}$. On reprend la notation $\tilde{G}(n)$ pour le produit semi-direct de $G L(n)$ avec le groupe engendré par $\theta$ et on note $G_{n}^{+}$la composante non neutre de ce groupe.

Si $n$ est pair, on note $H_{n}$ la forme déployée du groupe spécial orthogonal $S O(n+1)$ sur $F$. Si $n$ est impair, on note $H_{n}$ le groupe symplectique $S p(n-1)$ sur $F$. Plus exactement, $H_{n}$ est le groupe des points sur $F$ du groupe algébrique indiqué. Le groupe $H_{n}$ est un groupe endoscopique de $\tilde{G}(n)$, c'est celui qui contrôle les distributions stables sur $G_{n}^{+}$. Notons $G_{n, \text { reg }}^{+}$, resp. $H_{n, \text { reg }}$, l'ensemble des éléments semi-simples réguliers de $G_{n}^{+}$, resp. $H_{n}$. On sait définir une application, d'ailleurs bijective, entre classes de conjugaison stable dans $G_{n, \text { reg }}^{+}$et classes de conjugaison stable dans $H_{n, \text { reg }}$; cf. [11, [12] et pour une description concrète [26] III.2.

Pour $\tilde{g} \in G_{n, \text { reg }}^{+}$et $h \in H_{n, r e g}$, on note $\tilde{g} \sim h$ si les classes de conjugaison stable de $\tilde{g}$ et de $h$ se correspondent. Soient D, resp. $\mathbf{D}^{H}$, deux distributions sur $\tilde{G}(n)$, resp. $H_{n}$, invariantes par conjugaison par $G L(n)$, resp. $H_{n}$, et localement intégrables, c'est-à-dire définies par des fonctions localement intégrables notées $D$, resp. $D^{H}$. Supposons de plus $D$, resp. $D^{H}$, localement constantes sur $G_{n, r e g}^{+}$, resp. $H_{n, \text { reg }}$. Alors $\mathbf{D}$, resp. $\mathbf{D}^{H}$ sont stablement invariantes si et seulement si $D$, resp. $D^{H}$, est constante sur les classes de conjugaison stable contenues dans $G_{n, \text { reg }}^{+}$, resp. $H_{n, r e g}$. La distribution $\mathbf{D}$ est un transfert de $\mathbf{D}^{H}$ si et seulement si on a l'égalité $D(\tilde{g})=D^{H}(h)$ pour tout couple $(\tilde{g}, h) \in G_{n, \text { reg }}^{+} \times H_{n, \text { reg }}$ tel que $\tilde{g} \sim h$. En particulier, soient $\pi$, resp. $\pi^{H}$, des représentations virtuelles de $\tilde{G}(n)$, resp. $H_{n}$, c'est-àdire des combinaisons linéaires à coefficients dans $\mathbb{Z}$ de représentations irréductibles. Notons trace $\pi^{H}$ le caractère de $\pi^{H}$ (défini par linéarité à partir du cas où $\pi^{H}$ est irréductible). Notons $\operatorname{trace}_{G_{n}^{+}} \pi$ la restriction à $G_{n}^{+}$du caractère de $\pi$. Pour simplifier les notations, on considère ces caractères soit comme des distributions, soit comme des fonctions localement constantes sur $G_{n, \text { reg }}^{+}$ou $H_{n, r e g}$. On dit que $\pi$, resp. $\pi^{H}$, est stable si et seulement si $\operatorname{trace}_{G_{n}^{+}} \pi$ l'est, resp. trace $\pi^{H}$ l'est. On dit que $\pi$ est un transfert de $\pi^{H}$ si et seulement si trace $_{G_{n}^{+}} \pi$ est un transfert de $\operatorname{trace} \pi^{H}$.

\subsection{Propriétés générales du transfert stable}

Comme souvent quand on travail avec $G L(n), H_{n}$ on doit aussi travailler avec des Levi de ces groupes qui se correspondent; considérons une partition de $n$ de la forme symétrique suivante

$$
\left(n_{1}, \cdots, n_{r}, n_{0}, n_{r}, \cdots, n_{1}\right) ; n=\sum_{i \in[1, r]} 2 n_{i}+n_{0}
$$


On note $M$ le Levi standard de $G L(n)$ correspondant à cette partition et $M^{H}$ celui de $H_{n} ; M^{H}$ est isomorphe à $\times_{i \in[1, r]} G L\left(n_{i}\right) \times H_{n_{0}}$ et $M$ est $\theta$ invariant. Ceci permet de définir $\tilde{M}$ et $M^{+}$comme sous-groupe, resp. sous-ensemble de $\tilde{G}(n)$ et $G_{n}^{+}$. On fixe un ensemble $\mathcal{C}$ de représentations cuspidales (non autoduales en général). Pour $\pi$ une représentation de $\tilde{G}$, on définit $J a c_{\mathcal{C}}^{\theta} \pi$ de la façon suivante : on considère d'abord la restriction de $\pi$ le long du parabolique $P$ de Levi $M$. On obtient donc une représentation de $M$; on projette cette représentation sur son facteur direct tel que l'action des $r$ premiers (resp. derniers) facteurs $G L\left(n_{i}\right)$ se fasse via des représentations de support cuspidal les représentations de $\mathcal{C}$ (resp. ${ }^{\theta} \mathcal{C}$ ); on note cette opération $\operatorname{proj}_{\mathcal{C}}^{\theta}$. L'action de $\theta$ sur $\pi$ donne une action de $\theta$ sur cette projection et on obtient donc une représentation de $\tilde{M}$. Si $\pi^{H}$ est une représentation de $H$, on définit de façon analogue (et plus simple) $J a c_{\mathcal{C}} \pi^{H}$, ce qui utilise la projection $\operatorname{proj}_{\mathcal{C}}$. On étend ces définitions linéairement au groupe de Grothendieck des représentations de $\tilde{G}(n)$ et de $H$.

Lemme. Soient $\pi^{H}$ une représentation virtuelle de $H_{n}, \pi$ une représentation virtuelle de $\tilde{G}(n)$ et $M, M^{H}$ comme ci-dessus. Supposons que $\pi^{H}$ et $\pi$ sont stables et que $\pi$ est un transfert de $\pi^{H}$.

Alors $J a c_{\mathcal{C}} \pi^{H}$ et $J a c_{\mathcal{C}}^{\theta} \pi$ sont stables et $J a c_{\mathcal{C}}^{\theta} \pi$ est un transfert de $J a c_{\mathcal{C}} \pi^{H}$.

On démontre en 2 temps cette assertion; d'abord on démontre que les modules de Jacquet ont les propriétés de stabilité et de transfert annoncés puis on montre que leur projection suivant $\mathcal{C}$ ont aussi ces propriétés. C'est un résultat qui ne surprend personne mais dont on redonne la démonstration.

On note $\pi_{P}$ et $\pi_{P}^{H}$ les modules de Jacquet de $\pi$ et $\pi^{H}$ respectivement; on rappelle que $\theta$ agit sur $\pi_{P}$ et que $\pi_{P}$ est donc une représentation de $\tilde{M}$. Il suffit de montrer que, pour tous $\tilde{g} \in M_{\text {reg }}^{+}$et $h \in M_{\text {reg }}^{H}$ tels que $\tilde{g} \sim h$, on a l'égalité $\operatorname{trace}_{\tilde{M}} \pi_{P}^{+}(\tilde{g})=\operatorname{trace}_{P^{H}}^{H}(h)$. Fixons de tels $\tilde{g}$ et $h$. Ecrivons :

$$
\begin{gathered}
M=G L\left(n_{1}\right) \times \ldots \times G L\left(n_{r}\right) \times G L\left(n_{0}\right) \times G L\left(n_{r}\right) \times \ldots \times G L\left(n_{1}\right) \\
M^{H}=G L\left(n_{1}\right) \times \ldots \times G L\left(n_{r}\right) \times H_{n_{0}} .
\end{gathered}
$$

Soient $z_{1}, \ldots, z_{r} \in F^{\times}$, posons :

$$
\begin{gathered}
z_{M}=z_{1} \mathbf{1} \times \ldots \times z_{r} \mathbf{1} \times \mathbf{1} \times z_{r}^{-1} \mathbf{1} \times \ldots \times z_{1}^{-1} \mathbf{1}, \\
z_{M^{H}}=z_{1} \mathbf{1} \times \ldots \times z_{r} \mathbf{1} \times \mathbf{1},
\end{gathered}
$$

où on note uniformément 1 les unités des groupes en question. Supposons que les réels suivants soient assez petits :

- $\left|\frac{z_{i}}{z_{i+1}}\right|$ pour $i=1, \ldots, r-1$ et $\left|z_{r}\right|$.

Alors un résultat de Casselman affirme que l'on a les égalités :

$$
\begin{gathered}
\operatorname{trace}_{M^{+}} \pi_{P}\left(z_{M} \tilde{g}\right)=\delta_{P}\left(z_{M} \tilde{g}\right)^{-1 / 2} \operatorname{trace}_{G_{n}^{+}} \pi\left(z_{M} \tilde{g}\right), \\
\operatorname{trace}_{P^{H}}^{H}\left(z_{M^{H}} h\right)=\delta_{P^{H}}\left(z_{M^{H}} h\right)^{-1 / 2} \operatorname{trace} \pi^{H}\left(z_{M^{H}} h\right),
\end{gathered}
$$

où $\delta_{P}$ et $\delta_{P H}$ sont les modules usuels. De la définition de la correspondance entre classes de conjugaison stables et de l'hypothèse $\tilde{g} \sim h$ résulte que $z_{M} \tilde{g} \sim z_{M^{H}} h$. On vérifie par ailleurs l'égalité des facteurs $\delta$ ci-dessus. Puisque $\pi$ est un transfert de $\pi^{H}$, les membres de droite des égalités ci-dessus sont égaux. Donc les membres de gauche le sont aussi. Mais, parce que $z_{M}$ appartient au centre de $M^{+}$, la fonction :

$$
\left(z_{1}, \ldots, z_{r}\right) \mapsto \operatorname{trace}_{\tilde{M}} \pi_{P}\left(z_{M} \tilde{g}\right)
$$

est une combinaison linéaire finie de caractères de $\left(F^{\times}\right)^{r}$. Il en est de même de la fonction :

$$
\left(z_{1}, \ldots, z_{r}\right) \mapsto \operatorname{trace} \pi_{P^{H}}^{H}\left(z_{M^{H}} h\right) .
$$

On vient de voir que ces fonctions coïncident dans un "cône ouvert" de l'espace de départ. Il en résulte qu'elles sont égales. En particulier, leurs valeurs en $\left(z_{1}, \ldots, z_{r}\right)=(1, \ldots, 1)$ sont égales, c'est-à-dire trace $_{M^{+}} \pi_{P}(\tilde{g})=\operatorname{trace} \pi_{P^{H}}^{H}(h)$, ce que l'on voulait démontrer. 
On reprend les notations $\operatorname{proj}_{\mathcal{C}}^{\theta}$ et $\operatorname{proj}_{\mathcal{C}}$ introduites avant l'énoncé du lemme. Le point à démontrer est maintenant le suivant : soit $\pi_{M}$ une représentation virtuelle de $\tilde{M}$ et $\pi_{M}^{H}$ une représentation virtuelle de $M^{H}$; on suppose qu'elles sont stables et que $\pi_{M}$ est un transfert de $\pi_{M}^{H}$, alors la même propriété est vraie pour $\operatorname{proj}_{\mathcal{C}}^{\theta} \pi_{M}$ et $\operatorname{proj}_{\mathcal{C}} \pi_{M}^{H}$. On écrit $\pi_{M}$ dans la base des représentations irréductibles de $\tilde{M}$ et on remarque tout de suite que toute représentation irréductible de $\tilde{M}$ dont la restriction à $M$ n'est pas irréductible donne une trace nulle sur $M^{+}$; on peut donc supposer que

$$
\pi_{M}=\sum_{\sigma, \sigma_{0}} c\left(\sigma, \sigma_{0}\right) \sigma \otimes \sigma_{0} \otimes \theta(\sigma),
$$

où $\sigma$ parcourt l'ensemble des représentations irréductibles de $\times_{i \in[1, r]} G L\left(n_{i}\right)$ et $\sigma_{0}$ l'ensemble des représentations irréductibles de $\tilde{G}\left(n_{0}\right)$ et où $c\left(\sigma, \sigma_{0}\right)$ est un nombre complexe. On regroupe en fonction $\operatorname{des} \sigma$ :

$$
\pi_{M}=\sum_{\sigma} \sigma \otimes \sigma_{0}[\sigma] \otimes \theta(\sigma)
$$

où $\sigma$ est comme ci-dessus mais où $\sigma_{0}[\sigma]$ est une représentation virtuelle de $\tilde{G}\left(n_{0}\right)$; remarquons que si $n_{0}=0$ il reste quand même une action de $\theta$, c'est-à-dire un signe. On écrit de façon analogue

$$
\pi_{M}^{H}=\sum_{\sigma} \sigma \otimes \sigma_{0}^{H}[\sigma]
$$

On va démontrer que sous l'hypothèse que $\pi_{M}$ et $\pi_{M}^{H}$ sont stables et des transferts l'une de l'autre alors il en est de même de $\sigma_{0}[\sigma]$ et $\sigma_{0}^{H}[\sigma]$ pour tout $\sigma$ comme ci-dessus. Ce sera largement suffisant.

Soient $g=\left(g_{1}, \ldots, g_{r}, g_{0}, g_{r}^{\prime}, \ldots, g_{0}^{\prime}\right) \in M_{r e g}$ et $h=\left(h_{1}, \ldots, h_{r}, h_{0}\right) \in M_{r e g}^{H}$. On vérifie les propriétés suivantes :

- $g \theta \sim h$ si et seulement si $g_{0} \theta \sim h_{0}$ et, pour tout $i=1, \ldots, r, g_{i} \theta\left(g_{i}^{\prime}\right)$ est conjugué à $h_{i}$ dans $G L\left(n_{i}\right)$;

- pour toute représentation irréductible $\sigma=\sigma_{1} \otimes \ldots \otimes \sigma_{r}$ de $G L\left(n_{1}\right) \times \ldots \times G L\left(n_{r}\right)$ et pour toute représentation virtuelle $\sigma_{0}^{+}$de $G L\left(n_{0}\right)^{+}$, on a l'égalité :

$$
\left.\operatorname{trace}_{\tilde{M}}\left(\sigma \otimes \sigma_{0}^{+}\right)(g \theta)=\text { trace }_{G_{n_{0}}^{+}} \sigma_{0}^{+}\left(g_{0} \theta\right) \prod_{i=1, \ldots, r} \operatorname{trace}_{i}\left(g_{i} \theta\left(g_{i}^{\prime}\right)\right)\right) .
$$

Dire que $\pi_{M}$ et $\pi_{M}^{H}$ sont stables et que $\tau_{M}$ est un transfert de $\pi_{M}^{H}$ signifie donc que pour tout $\gamma \in G L\left(n_{1}\right) r e g \times \ldots \times G L\left(n_{r}\right) r e g$, tout $\tilde{g}_{0} \in G_{n_{0}}^{+}$, tout $h_{0} \in H_{n_{0}, \text { reg }}$ tels que $\tilde{g}_{0} \sim h_{0}$, on a l'égalité :

$$
\sum_{\sigma} \operatorname{trace} \sigma(\gamma) \operatorname{trace}_{G_{n_{0}}^{+}} \sigma_{0}^{+}[\sigma]\left(\tilde{g}_{0}\right)=\sum_{\sigma} \operatorname{trace} \sigma(\gamma) \operatorname{trace}_{0}^{H}[\sigma]\left(h_{0}\right) .
$$

Considérons $\tilde{g}_{0}$ et $h_{0}$ comme fixés et $\gamma$ comme variable. L'égalité ci-dessus est une égalité entre combinaisons linéaires (à coefficients complexes) de traces de représentations irréductibles de $G L\left(n_{1}\right) \times$ $\ldots \times G L\left(n_{r}\right)$. On sait que ces traces sont linéairement indépendantes. Ainsi cette égalité entraîne que, pour tout $\sigma$, on a l'égalité :

$$
\text { trace }_{G_{n_{0}}^{+}} \sigma_{0}^{+}[\sigma]\left(\tilde{g}_{0}\right)=\operatorname{trace}_{0}^{H}[\sigma]\left(h_{0}\right) .
$$

Ces égalités achèvent la démonstration.

\subsection{Les représentations}

Jusqu'à présent, nous avions regardé un ensemble de morphismes $\psi \theta$-invariant, il faut maintenant tenir compte des questions de parité qui sont liés aux choix de $H_{n}$ si l'on veut que l'objet attaché soit un transfert d'un analogue pour $H_{n}$. Pour $\rho$ une représentation cuspidale irréductible autoduale, on attache un signe $\eta_{\rho}$ de la façon suivante. D'après la conjecture de Langlands (théorème de HarrisTaylor et Henniart), on sait associer à $\rho$ une représentation irréductible de dimension $d_{\rho}$ du groupe de Weil de $F$. Parce que $\theta(\rho)=\rho$, l'image de cette représentation est incluse dans un sous-groupe de $G L_{d_{\rho}}(\mathbb{C})$ qui est soit un groupe orthogonal, soit un groupe symplectique. On pose $\eta_{\rho}=1$ dans 
le premier cas, $\eta_{\rho}=-1$ dans le second. On rappelle d'autre part que, pour toute représentation $\pi$ irréductible, on note $\chi_{\pi}$ son caractère central. L'hypothèse $\theta(\pi)=\pi$ entraîne $\chi_{\pi}^{2}=1$. Pour $\rho$ comme ci-dessus telle que $\eta_{\rho}=-1, \chi_{\rho}$ est nécessairement trivial. On va maintenant noter $\Psi_{n}^{H}$ l'ensemble des morphismes $\psi$ de $W_{F} \times S L(2, \mathbb{C}) \times S L(2, \mathbb{C})$ dans $G L(n, \mathbb{C})$ vérifiant les propriétés suivantes (les morphismes sont regardés à conjugaison près) :

1- l'image de $\psi$ est à valeurs dans $S p(n)$ si $n$ est pair et à valeurs dans $S O(n)$ si $n$ est impair. On note $H^{*}$ le groupe $S p(n)$ ou $S O(n)$, comme ci-dessus;

2- le centralisateur de $\psi$ dans $H^{*}$ est un groupe à centre fini.

Cela se traduit encore de façon combinatoire ainsi : $\psi$ définit une représentation de dimension $n$ de $W_{F} \times S L(2, \mathbb{C}) \times S L(2, \mathbb{C})$ que l'on décompose en sous-représentations irréductibles. Chaque sousreprésentation irréductible est associée à un triplet $(\rho, a, b)$ où $\rho$ est une représentation irréductible de $W_{F}$ et $a, b$ sont les dimensions des représentations irréductibles de $S L(2, \mathbb{C})$. La condition 1 assure que la multiplicité de la représentation associée au triplet $(\rho, a, b)$ dans cette décomposition est la même que celle de la représentation $\left(\rho^{*}, a, b\right)$. La condition 2 entraîne alors que $\rho \simeq \rho^{*}$ (sinon le centralisateur contient un groupe linéaire). Et la condition 1 peut alors se récrire :

$$
\eta_{\rho}(-1)^{a+b}=(-1)^{n+1} \quad \text { et } \quad \prod_{(\rho, a, b)} \chi_{\rho}^{a b}=1 .
$$

Remarque. Pour $\psi \in \Psi_{n}$, on a défini $\pi(\psi)$ comme représentation de $\tilde{G}(n)$. Cette définition dépend $d u$ choix initial de l'action de $\theta$ sur chaque $\rho$ intervenant dans la décomposition. Un choix raisonnable, c'est celui que nous avons fait, est de fixer un caractère additif $\tau$ est d'imposer que $\theta$ agisse trivialement sur l'espace des vecteurs de Whittaker associé à $\pi(\psi)$ (l'espace des coinvariants comme expliqué dans l'introduction). L'action est indépendante du choix de $\tau$.

Remplaçons $\tau$ par le caractère $x \mapsto \tau(z x)$ avec $z \in F^{\times}$. L'action de $\theta$ sur une représentation autoduale $\rho$ d'un groupe $G L\left(d_{\rho}\right)$ est alors multipliée par $\chi_{\rho}(z)^{d_{\rho}+1}$ (on applique les définitions). Pour démontrer l'assertion d'indépendance, il suffit de le faire dans le cas où $\psi$ est de restriction discrète à la diagonale. On reprend les définitions et le changement de $\zeta$ induit donc la multiplication par le signe :

$$
\prod_{(\rho, a, b) \in \operatorname{Jord}(\psi)} \chi_{\rho}(z)^{\left(d_{\rho}+1\right)(a-2[a / 2])(b-2[b / 2])}=\prod_{(\rho, a, b)} \chi_{\rho}(z)^{a b}=1
$$

par les conditions ci-dessus. D'où l'assertion.

Définition. On note $\Psi_{n}^{\text {temp }}$ le sous-ensemble de $\Psi$ formé des morphismes triviaux sur la 2e copie de $S L(2, \mathbb{C})$.

\subsection{Hypothèse}

On fixe un ensemble $\mathcal{E}$ de représentations irréductibles de $W_{F}$ et pour tout $n$, on note $\Psi_{n, \mathcal{E}}$ l'ensemble des éléments de $\Psi_{n}$ dont la restriction à $W_{F}$ est une somme de représentations isotypiques de type des représentations dans $\mathcal{E}$. On définit de façon évidente $\Psi_{n, \mathcal{E}}^{t e m p}$.

Hypothèse. Nous allons supposer à partir de maintenant que $\mathcal{E}$ est fixé de telle sorte que pour tout $n$ et tout $\phi \in \Psi_{n, \mathcal{E}}^{t e m p}$, la représentation tempérée, $\pi(\phi)$, associée à $\phi$ (grâce aux travaux de Zelevinsky et à la preuve de la conjecture de Langlands) est un transfert d'une représentation virtuelle $\pi^{H}(\phi)$ de $H_{n}$.

Remarquons que l'on n'a pas précisé l'action de $\theta$; il est clair que si l'on a un transfert pour un choix d'action de $\theta$, on en aura pour tout choix, la seule chose est que $\pi(\phi)$ n'est défini qu'au signe près. Toutefois, les représentations irréductibles de $H_{n}$ intervenant dans la décomposition de $\pi^{H}(\phi)$ sont uniquement déterminées par $\phi$ et l'égalité des traces. On notera $\Pi^{H}(\phi)$ cet ensemble de représentations. 


\subsection{Remarques sur l'hypothèse; points de réductibilité des induites de cuspidales}

On suppose que le lemme fondamental pour $H_{n}, \tilde{G}(n)$ est connu, les travaux d'Arthur démontrent alors cette hypothèse. Ces travaux la démontrent d'ailleurs sans supposer que $\phi$ est tempéré. Ce que nous faisons ici, ramener le cas général au cas tempéré, n'a donc d'intérêt que si l'on peut être plus précis et en particulier si on peut décomposer les représentations virtuelles obtenues. Sans connaissance précise de $\pi^{H}(\phi)$ dans le cas cas tempéré, nous n'ajoutons pas grand chose.

Ici, nous allons voir rapidement ce que l'on peut en déduire de cette hypothèse très générale et reposer le problème précisément.

Remarque 1. Arthur annonce en [6] 30.1 que pour un bon choix de l'action de $\theta, \pi^{H}(\phi)$ est pour tout $\phi$ une combinaison linéaire à coefficients positifs de représentations. Alors $\pi^{H}(\phi)$ est une représentation tempérée.

En effet, les modules de Jacquet non nul de $\pi^{H}(\phi)$ se transfèrent en des modules de Jacquet non nuls de $\pi(\phi)$ d'après ce que l'on a vu en 4.2 et il est alors facile de vérifier la positivité des exposants. On peut conclure que chaque représentation irréductible constituant $\pi^{H}(\phi)$ est tempérée car il ne peut y avoir de simplification dans le calcul des modules de Jacquet sous l'hypothèse faite que $\pi^{H}(\phi)$ est une représentation et pas seulement une représentation virtuelle. Une démonstration plus conceptuelle est dans [26] VI.1.

Remarque 2. Soit $\sigma$ une représentation cuspidale de $H_{n}$; supposons qu'il existe un morphisme $\phi \in \Psi_{n, \mathcal{E}}^{\text {temp }}$ tel que $\sigma \in \Pi^{H}(\phi)$. Alors

(i) Jord $(\phi)$ est sans multiplicités et sans trou, c'est-à-dire que si une représentation $\rho \otimes[a]$ de $W_{F} \times S L(2, \mathbb{C})([a]$ est la représentation de dimension a de $S L(2, \mathbb{C}))$ intervient dans la représentation définie par $\phi$, elle y intervient avec multiplicité 1 et si $a>2$ la représentation $\rho \otimes[a-2]$ y intervient aussi avec multiplicité 1 .

(ii) Soit $\rho$ une représentation irréductible cuspidale de $G L\left(d_{\rho}, F\right)$ (ce qui définit $d_{\rho}$ ). On suppose que $\rho \simeq \rho^{*}$ et que l'ensemble $\operatorname{Jord}_{\rho}(\phi) \neq 0$ et on note $a_{\max }$ son élément maximum. Soit $x_{0} \in \mathbb{R}$ tel que l'induite $\rho||^{x_{0}} \times \sigma$ soit réductible. Alors $x_{0}= \pm\left(a_{\max }+1\right) / 2$.

(iii)Soit $\rho$ comme en (ii) mais tel que $\operatorname{Jord}_{\rho}(\phi)=\emptyset$; on suppose que $\eta_{\rho}=(-1)^{n+1}$, alors pour $x_{0}$ comme en (ii), $x_{0}= \pm 1 / 2$.

Avant de donner la preuve, remarquons que l'énoncé ne dit rien si $\eta_{\rho}=(-1)^{n}$ et l'ensemble de (ii) est vide. On traitera ce cas dans [17]. Toutefois, les propriétés démontrées ici sont suffisantes pour construire avec [18], [19], pour tout morphisme $\phi \in \Psi_{n, \mathcal{E}}^{\text {temp }}$ discret (i.e. Jord $\phi$ ) est sans multiplicités) une application (non triviale) de l'ensemble des caractères du centralisateur de $\phi$ dans ${ }^{L} H_{n}, \epsilon \mapsto$ $\pi^{H}(\phi, \epsilon)$, avec $\pi^{H}(\phi, \epsilon)$ est soit 0 soit une série discrète sans multiplicités. Les questions sont alors :

1- a-t-on $\Pi^{H}(\phi)=\left\{\pi^{H}(\phi, \epsilon)\right\}$ ? la réponse est nécessairement oui si l'on sait a priori que les $c_{\pi}$ sont des réels positifs pour un bon choix de l'action de $\theta$, ce qui fait partie des résultats d'Arthur déjà cités.

2- a-t-on plus précisément $\operatorname{tr} \pi(\phi)(g, \theta)= \pm \sum_{\epsilon} \operatorname{tr} \pi^{H}(\phi, \epsilon)(h)$ quand les classes de conjugaison stable de het de $(g, \theta)$ se correspondent? C'est cette formule que nous a en vue avec une description précise des représentations $\pi^{H}(\phi, \epsilon)$.

Supposons d'abord que $\operatorname{Jord}(\phi)$ ne soit pas sans multiplicités; il existe donc $\rho \otimes[a]$ qui intervient dans $\phi$ avec multiplicité au moins 2 (notations de l'énoncé). On décompose :

$$
\phi=\phi_{0} \oplus \rho \otimes[a] \oplus \rho \otimes[a] .
$$

Pour $n_{0}$ convenable, $\phi_{0} \in \Psi_{n_{0}, \mathcal{E}}^{t e m p}$. On vérifie que pour tout $g \in \tilde{G}_{n, r e g}^{+}$

$$
\operatorname{tr} \pi(\phi)(g)=\operatorname{tr} S t(a, \rho) \times \pi\left(\phi_{0}\right) \times \operatorname{St}(a, \rho)(g)
$$

qui dans le transfert donne une égalité en tout point $h \in H_{n, r e g}$

$$
\operatorname{tr} \pi^{H}(\phi)(h)=\operatorname{tr}\left(\operatorname{ind}\left(S t(a, \rho) \otimes \pi^{H_{n_{0}}}\left(\phi_{0}\right)\right)(h) .\right.
$$


Ainsi $\Pi^{H}(\phi)$ est inclus dans la réunion des composantes irréductibles des induites de la forme $S t(a, \rho) \times$ $\sigma_{0}$ pour $\sigma_{0} \in \Pi^{H_{n}}\left(\phi_{0}\right)$. A priori, il pourrait y avoir des simplifications et c'est pour cela que l'on n'a qu'une inclusion. Mais comme $\sigma$ est cuspidale par hypothèse, elle ne pourrait être dans $\Pi^{H}(\phi)$. D'où la première assertion de (i).

Montrons le reste de la remarque : on fixe, en admettant que c'est possible, $(\rho, a)$ avec $a \geq 0$ tel que $\rho \otimes[a+2]$ ne soit pas une sous-représentation de de $\phi$ (i.e. $(\rho, a+2) \notin \operatorname{Jor} d(\phi))$ alors que $\rho \otimes[a]$ en est une si $a>0$; si $a=0$ on suppose que $\eta_{\rho}=(-1)^{n+1}$. On note $\phi_{1}$ le morphisme qui se déduit de $\phi$ de sorte que

$$
\operatorname{Jord}\left(\phi_{1}\right)=\operatorname{Jord}(\phi)-(\rho, a) \cup(\rho, a+2) .
$$

Clairement $\phi_{1} \in \Psi_{n+2 d_{\rho}, \mathcal{E}}^{t e m p}$. On calcule (les Jac sont comme en 1.4 pour $\rho$ fixé) :

$$
\operatorname{Jac}_{(a+1) / 2}^{\theta} \pi\left(\phi_{1}\right)=\pi(\phi) ; \quad J_{a c_{-(a+1) / 2}^{\theta}}^{\theta}\left(\phi_{1}\right)=0 .
$$

En particulier $J a c_{(a+1) / 2} \pi^{H}\left(\phi_{1}\right)=\pi^{H}(\phi)$ et $J a c_{-(a+1) / 2} \pi^{H}\left(\phi_{1}\right)=0$. Ainsi, il existe $\sigma_{1}$ dans $\Pi^{H}\left(\phi_{1}\right)$ tel que $J_{a c_{(a+1) / 2} \sigma_{1}}$ contient $\sigma$ comme sous-quotient. Ainsi $\sigma_{1}$ est un sous-module irréductible de l'induite $\rho \|^{(a+1) / 2} \times \sigma$. Si cette induite est irréductible, $\sigma_{1}$ coïncide avec toute l'induite et est donc l'unique élément de $\Pi^{H}\left(\phi_{1}\right)$ sous-quotient de cette induite. Et on ne pourrait avoir de simplification de $J a c_{-(a+1) / 2} \sigma_{1}$ quand on calcule $J a c_{-(a+1) / 2} \pi^{H}\left(\phi_{1}\right)$. On vient donc de montrer que $\left.\rho\right|^{(a+1) / 2} \times \sigma$ est réductible. D'après un résultat de Silberger (24]) $(a+1) / 2$ est uniquement déterminé par $\rho$ et $\sigma$ (il n'y a qu'un point de réductibilité réel positif ou nul).

Supposons maintenant que $\rho$ est tel que $\operatorname{Jord}_{\rho}(\phi) \neq 0$. En prenant $a=a_{\text {max }}$ l'élément maximum de $\operatorname{Jord}_{\rho}(\phi)$, l'hypothèse $(\rho, a+2) \notin \operatorname{Jord}(\phi)$ est bien satisfaite; ainsi $\left(a_{\max }+1\right) / 2$ est le point de réductibilité cherché. L'inégalité prouvée en [20] (cf. l'intorduction de cet article) montre que $\phi$ est sans trou et on a prouvé (ii). On démontre (iii) en prenant $a=0$. Cela termine la preuve.

\subsection{Construction des représentations de $H_{n}$}

On fixe $\psi \in \Psi_{n, \mathcal{E}}$ avec l'hypothèse faite en 4.4; pour pouvoir traduire en termes combinatoires, on décompose la représentation $\psi$ de $W_{F} \times S L(2, \mathbb{C}) \times S L(2, \mathbb{C})$ en représentations irréductibles, d'où un ensemble de triplets $(\rho, a, b)$ constituant par définition $\operatorname{Jor} d(\psi)$. On rappelle que $\psi \circ \Delta$ est la restriction de $\psi$ à $W_{F}$ fois la diagonale de $S L(2, \mathbb{C})$. Il est facile de vérifier que le centralisateur de $\psi$ dans $G L(n, \mathbb{C})$ est un sous-groupe du centralisateur de $\psi \circ \Delta$. On dit que $\psi$ est discret si le centralisateur de $\psi$ est un groupe fini et que $\psi$ est de restriction discrète à la diagonale si le centralisateur de $\psi \circ \Delta$ est un groupe fini (notation déjà utilisée ici). On verra souvent $\psi \circ \Delta$ comme un élément de $\Psi_{n, \mathcal{E}}^{t e m p}$.

On suppose d'abord que $\Psi$ est élémentaire, discret, c'est-à-dire par définition que l'inclusion du centralisateur de $\psi$ dans le centralisateur de $\psi \circ \Delta$ est un isomorphisme et que ces groupes sont finis. En termes combinatoires cela se traduit par le fait que $\operatorname{Jord}(\psi)$ est sans multiplicités et que pour tout $(\rho, a, b) \in \operatorname{Jord}(\psi), \inf (a, b)=1$. On sait donc parfaitement définir $\pi^{H}(\psi \circ \Delta)$ grâce à l'hypothèse de 4.4. Pour construire $\pi^{H}(\psi)$ en suivant [14], on a besoin de la construction $i n v_{\rho, \leq d}$, où $\rho$ est une cuspidale autoduale irréductible d'un groupe linéaire et $d$ est un entier strictement supérieur à 1 et où pour toute représentation $\pi^{H}$ de $H_{n}$, on pose, $\mathcal{C}_{\rho, \leq d}$ l'ensemble des représentations cuspidales de la forme $\rho||^{x}$ avec $x$ un réel vérifiant $|x| \leq(d-1) / 2$ et

$$
\operatorname{inv}_{\rho, \leq d}:=\sum_{P^{H}}(-1)^{\operatorname{corang}^{H}} \operatorname{Ind}_{P^{H}}^{H_{n}} \operatorname{Jac}_{\mathcal{C}_{\rho, \leq d}}^{P^{H}} \pi^{H} .
$$

On donne une définition analogue pour $<d$ en remplaçant partout $\leq$ par $<$. On définit alors

$$
\pi^{H}(\psi):=\circ_{(\rho, a, b) \in \operatorname{Jord}(\psi) ; \sup (a, b)=b}\left(\beta(\psi, \rho, b) i n v_{\rho,<b} \circ i n v_{\rho, \leq b}\right) \pi^{H}(\psi \circ \Delta),
$$

où $\beta(\psi, \rho, b)$ est un signe que l'on précisera ci-dessous et où on prend n'importe quel ordre pour faire les opérations; en [14], on a bien montré que l'ordre n'importait pas mais avec des hypothèses un 
peu différentes (a priori au moins) de celles que l'on a ici. Comme on va montrer que quel que soit l'ordre $\pi^{H}(\psi)$ est stable et que $\pi(\psi)$ en est un transfert, on aura bien l'indépendance de l'ordre. Le signe n'est pas simple à interpréter, il vaut $\beta(\psi, \rho, \leq b) \beta\left(\psi, \rho, \leq b^{\prime}\right)$ où ces signes ont été définis en 3.2 avec où $b^{\prime}$ est le plus grand entier s'il existe (sinon le signe correspondant est + ) tel qu'il existe $\left(\rho, a^{\prime \prime}, b^{\prime \prime}\right) \in \operatorname{Jord}(\psi)$ avec $\sup \left(a^{\prime \prime}, b^{\prime \prime}\right)=b^{\prime}<b$.

Dans ce travail, nous n'avons besoin que de la définition mais pour motiver un peu rappelons le résultat de [14]. En fait tel que cet article est rédigé on a supposé un peu plus que seulement 4.4; on a supposé qu'il existe une bijection entre l'ensemble des caractères cuspidaux du centralisateur d'un morphisme discret sans trou, $\phi$, (cf. 4.5) et les représentations cuspidales incluses dans $\Pi^{H}(\phi)$. On pourrait facilement reformuler les résultats pour éviter cette dernière hypothèse ; il faut remplacer irréductible par nul ou de longueur finie sans multiplicités; ici on va continuer avec les hypothèses de [14] qui sont précisément cette asssertion de bijectivité, l'hypothèse 4.4 et une réponse positive à la question 2 de 4.5 .

En [14], on a alors montré que $\pi^{H}(\psi)$ est de la forme $\sum_{\epsilon} \epsilon\left(z_{2}\right) \pi^{H}(\psi, \epsilon)$ où $\epsilon$ parcourt l'ensemble des caractères du centralisateur de $\psi$ de restriction triviale au centre de ${ }^{L} H$, où $\pi^{H}(\psi, \epsilon)$ est une représentation irréductible et $z_{2}$ est le centre de la 2 e copie de $S L(2, \mathbb{C})$.

On suppose maintenant que $\psi$ est de restriction discrète à la diagonale. On reprend les notations bien commodes $(\rho, A, B, \zeta)$ de tout ce travail pour décrire les éléments de $\operatorname{Jord}(\psi)$ et on définit par récurrence $\pi^{H}(\psi)$, la récurrence porte sur $\sum_{(\rho, A, B, \zeta)}(A-B)$. On fixe $(\rho, A, B, \zeta) \in J$ ord $(\psi)$ tel que $A>B$, a priori la construction dépend de ce choix mais comme ci-dessus à posteriori elle n'en dépend pas, donc on ne s'ennuie pas avec cela et on pose :

$$
\begin{aligned}
& \pi^{H}(\psi):=\oplus_{C \in] B, A]}(-1)^{A-C}<\rho||^{\zeta C}, \cdots,\left.\rho\right|^{\zeta A}>\times J a c_{\rho||^{\zeta(B+2)}, \cdots, \rho||^{\zeta C}} \pi^{H}\left(\psi^{\prime},(\rho, A, B+2, \zeta)\right) \\
& \oplus(-1)^{[(A-B+1) / 2]} \pi^{H}\left(\psi^{\prime},(\rho, A, B+1, \zeta),(\rho, B, B, \zeta)\right) .
\end{aligned}
$$

En [15], on a décrit cette représentation virtuelle comme combinaison linéaire explicite de représentations indexées par les caractères du centralisateur de $\psi$, sous certaines hypothèses, le résultat obtenu (qui nécessite les mêmes hypothèses que dans le cas élémentaire) est que cette distribution s'écrit elle aussi sous la forme $\sum_{\epsilon} \epsilon\left(z_{2}\right) \pi^{H}(\psi, \epsilon)$ où chaque $\pi^{H}(\psi, \epsilon)$ est une représentation en général non irréductible. Une décomposition précise est donnée en loc.cit. Ici comme ci-dessus, nous n'avons besoin que de la définition.

On supprime encore l'hypothèse $\psi$ de restriction discrète à la diagonale, en fixant $N>>n$ et un morphisme $\tilde{\psi} \in \Psi_{N, \mathcal{E}}$ dominant $\psi$ suivant la définition de 2.4. On a alors défini en 2.4 l'ensemble de représentations $\mathcal{E}_{\tilde{\psi}}$ et on définit $J a c_{\mathcal{E}_{\tilde{\psi}}}$ comme en loc. cit. (attention, il faut tenir compte de l'ordre comme indiqué) et on pose :

$$
\pi^{H}(\psi):=J_{a c} \mathcal{E}_{\tilde{\psi}} \pi^{H}(\tilde{\psi}) .
$$

En [16], on décrit un peu moins précisément que dans le cas de restriction discrète à la diagonale cette représentation virtuelle, sous les mêmes hypothèses que ci-dessus, on garde toujours le même résultat que cette distribution s'écrit sous la forme $\sum_{\epsilon} \epsilon\left(z_{2}\right) \pi^{H}(\psi, \epsilon)$ où chaque $\pi^{H}(\psi, \epsilon)$ est une représentation en général non irréductible qui peut même ici être nulle.

\subsection{Preuve du transfert}

On fait ici l'hypothèse de 4.4 et on reprend les définitions de 4.6; on garde l'action de $\theta$ mise dans tout ce travail sur $\pi(\psi)$ pour tout $\psi \in \Psi_{n}$. On a en vue le théorème suivant :

Théorème. Les représentations $\pi(\psi)$ et $\pi^{H}(\psi)$ sont stables et $\psi(\psi)$ est un transfert de $\pi^{H}(\psi)$.

Remarquons que l'assertion de stabilité est un corollaire immédiat de 4.2 et du fait facile avec les formules explicites que l'induction respecte la stabilité. 
Ici on admet le théorème dans le cas élémentaire, le cas général se ramène au cas de restriction discrète à la diagonale grâce à 2.4 et à 4.2 . Le cas de restriction discrète à la diagonale résulte de 2.3 et 4.2 . Il n'y a donc à démontrer le théorème que dans le cas élémentaire.

Supposons donc que $\psi$ est élémentaire. On veut montrer que $\pi(\psi)$ est un transfert de $\pi^{H}(\psi)$, sous l'hypothèse que $\pi(\psi \circ \Delta)$ est un transfert de $\pi^{H}(\psi \circ \Delta)$. Si $\psi$ est trivial sur la 2e copie de $S L(2, \mathbb{C})$, il n'y a donc rien à démontrer. On fait donc la preuve par récurrence sur le nombre d'éléments de $\operatorname{Jord}(\psi),(\rho, a, b)$ tel que $\sup (a, b)=b>1$. On suppose que $\psi$ est de restriction discrète à la diagonale et on fixe $(\rho, a, b) \in \operatorname{Jord}(\psi)$ tel que $\sup (a, b)=b>1$. On a défini $\psi^{\sharp}$ en 3.1 .2 en prenant $d=b$. Puis on passe de $\psi^{\sharp}$ à $\left(\psi^{\sharp}\right)^{\sharp}$ en prenant maitenant $d$ le plus grand entier, notée $d_{0}$, strictement inférieur à $b$ et tel qu'il existe $d^{\prime}$ avec soit $\left(\rho, d, d^{\prime}\right) \in \operatorname{Jord}(\psi)$ soit $\left(\rho, d^{\prime}, d\right) \in \operatorname{Jor} d(\psi)$; si ce nombre n'existe pas, on pose $\left(\psi^{\sharp}\right)^{\sharp}=\psi^{\sharp}$. On a que $\operatorname{Jord}\left(\left(\psi^{\sharp}\right)^{\sharp}\right)$ coïncide avec $\operatorname{Jord}(\psi)$ sauf que $(\rho, a, b)$ a été changé en $(\rho, b, a)$. Par récurrence, on peut donc admettre que $\pi\left(\left(\psi^{\sharp}\right)^{\sharp}\right)$ est un transfert de $\pi^{H}\left(\left(\psi^{\sharp}\right)^{\sharp}\right)$, attention avec l'action que nous avons mise sur ces représentations. En tenant compte de ce qu'être un transfert commute à l'induction est à la restriction, on voit que $\pi\left(\psi^{\sharp}\right)$ est un transfert de $i n v_{\rho, \leq d_{0}} \pi^{H}\left(\left(\psi^{\sharp}\right)^{\sharp}\right)$ si $\beta\left(\psi \circ \Delta, \rho, \leq d_{0}\right)=+1$ et de son opposé sinon. Puis $\pi(\psi)$ est un transfert de $i n v_{\rho, \leq b} \circ i n v_{\rho, \leq d_{0}} \pi^{H}\left(\left(\psi^{\sharp}\right)^{\sharp}\right)$ si $\beta(\psi \circ \Delta, \rho, \leq b) \beta\left(\psi \circ \Delta, \rho, \leq d_{0}\right)=1$ et de son opposé sinon. En tenant compte de ce signe, on a exactement $\pi^{H}(\psi)$ tel qu'on l'a défini dans 4.6. Cela termine donc la preuve.

\section{$5 \quad$ Normalisation de l'action de $\theta$}

\subsection{Définition de la normalisation de Whittaker}

On fixe $\delta$ un caractère de $F$; si $\psi$ est tempéré, la représentation $\pi(\psi)$ a un modèle de Whittaker et l'action de $\theta$ est celle qui est normalisée à la Whittaker. On considère le morphisme de

$$
\begin{gathered}
W_{F} \times S L(2, \mathbb{C}) \rightarrow W_{F} \times S L(2, \mathbb{C}) \times S L(2, \mathbb{C}) \\
\left(w \in W_{F}, h \in S L(2, \mathbb{C})\right) \mapsto\left(w, h,\left(\begin{array}{cc}
|w|^{1 / 2} & 0 \\
0 & ||^{-1 / 2}
\end{array}\right)\right)
\end{gathered}
$$

et en composant on obtient un morphisme, $\psi^{2}$, de $W_{F} \times S L(2, \mathbb{C})$ dans $G L(n, \mathbb{C})$. A ce morphisme correspond une représentation par la classification de Langlands, qui n'est autre que $\pi(\psi)$; mais dans la construction de Langlands, $\pi(\psi)$ est quotient d'une induite à partir d'une représentation tempérée. Il est facile de voir que cette induite est $\theta$-invariante. On normalise l'action de $\theta$ sur l'induite en utilisant les fonctionnelles de Whittaker (cf. introduction).

Par restriction ce choix donne une action sur $\pi(\psi)$. Pour $z= \pm$, on note $\pi(\psi)^{z}$ la représentation $\pi(\psi)$ munie de cette action de $\theta$ si $z=+$ et de l'action opposée si $z=-$. Cette normalisation, facile à présenter n'est pas très simple non plus mais elle a le gros avantage par rapport à celle utilisée ici d'être d'origine globale. Localement il introduit des signes qu'il nous faut de toute façon calculer. On note $\pi(\psi)_{W}$ la représentation $\pi(\psi)$ avec l'action de $\theta$ telle que l'on vient de la définir et on note $\theta_{W}(\psi)$ cette action.

\subsection{Explicitation de la normalisation de Whittaker}

On écrit l'induite dont $\pi(\psi)$ est l'unique sous-module irréductible (on transforme quotient de Langands en sous-module sans problème). Pour cela, pour tout $k$ demi-entier positif ou nul, on définit $\operatorname{Jord}(\psi)_{\geq k}:=\{(\rho, a, b) \in \operatorname{Jord}(\psi) ;(b-1) / 2-k$ est un entier positif ou nul $\}$. Ainsi $\pi(\psi)$ est l'unique sous-module irréductible de l'induite :

$$
\left(\times_{k} \times\left._{(\rho, a, b) \in \operatorname{Jord}(\psi)_{\geq k}} \operatorname{St}(a, \rho)\right|^{-k}\right) \times_{(\rho, a, b) \in \operatorname{Jord}(\psi)_{\geq 0}} \operatorname{St}(a, \rho)\left(\times_{k} \times\left._{(\rho, a, b) \in \operatorname{Jord}(\psi)_{\geq k}} \operatorname{St}(a, \rho)\right|^{k}\right),
$$

où les $k$ sont d'abord pris dans l'ordre décroissant puis dans l'ordre croissant. Et l'action $\theta_{W}$ sur $\pi(\psi)$ normalisée "à la Whittaker" se déduit de l'action de $\theta$ sur cette induite normalisée à la Whittaker. On 
remarque que l'induite du milieu, correspondant à $k=0$, n'est autre que $\prod_{(\rho, a, b) \in \operatorname{Jord}(\psi) ;(-1)^{b}=-1} S t(a, \rho)$; on la note de façon cohérente avec ce qui précède $\pi\left(\psi_{i m p}^{2}\right)$. L'action de $\theta$ sur cette représentation est avec les notations déjà introduites $\theta_{W}\left(\psi_{i m p}^{2}\right)$.

Lemme. L'action $\theta_{W}(\psi)$ sur (1) est celle qui se déduit canoniquement de l'action de $\theta$ sur $\pi\left(\psi_{\text {imp }}^{2}\right)$.

Notons $V^{\prime}$ le sous-espace de l'induite (1) formé des fonctions à support dans la grosse cellule $P w B$ où $w$ est l'élément du groupe de Weyl de longueur maximal ( $P$ est le parabolique qui sert dans l'induction et $B$ le Borel standard). La restriction d'une fonctionnelle de Whittaker à $V^{\prime}$ se fait par l'intégration, où il faut fixer une fonctionnelle de Whittaker, $\ell_{M}$ sur la représentation que l'on induit, $\theta$ invariante (ce qui est possible)

$$
f \in V^{\prime} \mapsto \int_{U \cap M \backslash U} \ell^{M}(f(w u)) \chi(u)^{-1} d u .
$$

L'action canonique de $\theta$ laisse stable $V^{\prime}$ et cette forme linéaire, ce qui prouve le lemme.

On peut encore expliciter un peu plus; on note $\psi_{i m p, i m p}$ le morphisme dont les blocs de Jordan sont les $(\rho, a-2[a / 2], b-2[b / 2])$ pour $(\rho, a, b)$ parcourant $\operatorname{Jord}(\psi)$ (avec les mêmes multiplicités). On a encore une inclusion :

$$
\begin{aligned}
& \pi\left(\psi_{i m p}^{2}\right) \hookrightarrow \\
& \left(\times_{k \geq 1 / 2} \times(\rho, a, b) \in \operatorname{Jord}(\psi) ;\left.\rho\right|^{k}\right) \times \pi\left(\psi_{\text {imp }, i m p}\right) \times\left(\times(\rho, a, b) \in \operatorname{Jord}(\psi) ; \rho \|^{-k}\right), \\
& (-1)^{b}=-1 \\
& (a-1) / 2-k \in \mathbb{Z}_{\geq 0} \\
& (-1)^{b}=-1 \\
& (a-1) / 2-k \in \mathbb{Z}_{\geq 0}
\end{aligned}
$$

où dans le premier produit les $k$ sont les demi-entiers, $>0$, pris dans l'ordre décroissant et dans le deuxième produit les $k$ sont les demi-entiers, $>0$, pris dans l'ordre croissant. On obtient alors avec (1) une série d'inclusion, pour des représentations $\sigma, \sigma^{\prime}$ convenables qui sont explicitées dans (1) et (2)

$$
\pi(\psi) \hookrightarrow \sigma \times \pi\left(\psi_{i m p}^{2}\right) \times{ }^{\theta} \sigma \hookrightarrow \sigma \times \sigma^{\prime} \times \pi\left(\psi_{i m p, i m p}\right) \times{ }^{\theta} \sigma^{\prime} \times{ }^{\theta} \sigma .
$$

En suivant les définitions $\theta_{W}(\psi)$ est la restriction à $\pi(\psi)$ de l'action de $\theta$ sur le dernier membre de (3) qui se déduit canoniquement de $\theta_{W}\left(\pi(\psi)_{i m p, i m p}\right)$.

\subsection{Définition de la normalisation unipotente}

Dans la définition de la normalisation pour l'action de $\theta$ à la Whittaker, le côté tempéré, c'est-àdire la première copie de $S L(2, \mathbb{C})$ a été privilégié. D'un point de vue local, il n'y a pas de raison à ce choix; pour faire le choix opposé, il faut d'abord traiter le cas des représentations "unipotentes" c'est-à-dire les duales au sens de l'involution de Zelevinsky des représentations tempérées. Pour celleslà, on prend la normalisation à la Whittaker, c'est-à-dire qu'on représente cette représentation comme quotient de Langlands d'une série principale généralisée qui elle a un modèle de Whittaker. On fixe l'action de $\theta$ sur cette série principale en demandandant que $\theta$ induise l'action naturelle sur le modèle de Whittaker. Fixons maintenant $\psi$ général et on considère le morphisme de $W_{F}$ dans la première copie de $S L(2, \mathbb{C})$ analogue à celui défini en 5.1 que l'on note $i_{1}$ et on obtient un morphisme, $\psi_{\text {unip }}$ de $W_{F} \times S L(2, \mathbb{C})$ qui sur $W_{F}$ cö̈ncide avec $\psi \circ i_{1}$ sur $W_{F}$ et avec la restriction de $\psi$ à la deuxième copie de $S L(2, \mathbb{C})$ sur $S L(2, \mathbb{C})$. Ce morphisme $\psi_{\text {unip }}$ définit une induite à partir d'une représentation de Speh d'un Levi, c'est la classification originelle de Zelevinsky. Explicitons; on pose ici pour distinguer de $5.2 \operatorname{Jord}^{a}(\psi)_{\geq k}:=\left\{(\rho, a, b) \in \operatorname{Jord}(\psi) ;(a-1) / 2-k \in \mathbb{Z}_{\geq 0}\right\}$ pour tout $k$ demi-entier strictement positif et on pose $\psi_{i m p}^{1}$ le morphisme dont les blocs de Jordan sont les $(\rho, a-2[a / 2], b)$ pour tout $(\rho, a, b) \in \operatorname{Jord}(\psi)$ avec $a$ impair. Alors

$\pi(\psi) \hookrightarrow\left(\times_{k \geq 1 / 2} \times_{(\rho, a, b) \in \operatorname{Jord}^{a}(\psi)_{\geq k}} S p(b, \rho)||^{-k}\right) \times \pi\left(\psi_{\text {imp }}^{1}\right) \times\left(\times_{k \geq 1 / 2} \times_{(\rho, a, b) \in \operatorname{Jord}^{a}(\psi)_{\geq k}} S p(b, \rho)||^{k}\right)$, 
où les $k$ sont d'abord pris dans l'ordre décroissant puis dans l'ordre croissant. Comme on a mis une action de $\theta \operatorname{sur} \pi\left(\psi_{\text {imp }}^{1}\right)$, on prolonge canoniquement cette action $\operatorname{de} \theta \operatorname{sur}(1)$. Par restriction on obtient une action de $\theta$ sur $\pi(\psi)$ que l'on note $\theta_{U}(\psi)$.

Comme pour $\theta_{W}(\psi)$ on peut retrouver cette action par une suite d'inclusion analogue à (3) de 5.2; pour cela on considère l'inclusion

$$
\begin{aligned}
& \pi\left(\psi_{i m p}^{1}\right) \hookrightarrow \\
& \times_{k \geq 1 / 2} \times\left.(\rho, a, b) \in \operatorname{Jord}(\psi) \rho\right|^{-k} \times \pi\left(\psi_{i m p, i m p}\right) \times_{k \geq 1 / 2} \times(\rho, a, b) \in \operatorname{Jord}(\psi) \rho||^{k} \text {, } \\
& (-1)^{a}=-1 ; \quad(-1)^{a}=-1 ; \\
& (b-1) / 2-k \in \mathbb{Z}_{\geq 0} \quad(b-1) / 2-k \in \mathbb{Z}_{\geq 0}
\end{aligned}
$$

D'où pour des bons choix de $\tau, \tau^{\prime}$

$$
\pi(\psi) \hookrightarrow \tau \times \pi\left(\psi_{i m p}^{1}\right) \times{ }^{\theta} \tau \hookrightarrow \tau \times \tau^{\prime} \times \pi\left(\psi_{i m p, i m p}\right) \times{ }^{\theta} \tau^{\prime} \times{ }^{\theta} \tau .
$$

Et $\theta_{U}(\psi)$ s'obtient en restreignant l'action de $\theta$ sur le dernier membre de (3) obtenu en prolongeant canoniquement $\theta_{W}\left(\psi_{i m p, i m p}\right)$. C'est bien $\theta_{W}\left(\psi_{i m p, i m p}\right)$ car $\pi\left(\psi_{i m p, i m p}\right)$ est une induite de cuspidales et les choix se voient sur l'espace de Whittaker relatif au caractère additif que nous avons fixé. Pour garder la symétrie on pose

$$
\theta\left(\psi_{i m p, i m p}\right):=\theta_{U}\left(\psi_{i m p, i m p}\right):=\theta_{W}\left(\psi_{i m p, i m p}\right) .
$$

\subsection{Une propriété commune à ces actions}

Proposition. Soit $\psi$ et soit $(\rho, a, b) \in \operatorname{Jord}(\psi)$.

(i) Supposons que $b \geq 2$; on note $\psi^{\prime}$ le morphisme qui se déduit de $\psi$ en remplaçant $(\rho, a, b)$ par $(\rho, a, b-2)$. On suppose qu'il existe une inclusion :

$$
\pi(\psi) \hookrightarrow S t(a, \rho)||^{-(b-1) / 2} \times \pi\left(\psi^{\prime}\right) \times S t(a, \rho)||^{(b-1) / 2} .
$$

Alors $\theta_{W}(\psi)$ est par restriction l'action $\theta_{W}\left(\psi^{\prime}\right)$ étendue canoniquement à l'induite de droite.

(ii) Supposons que $a \geq 2$; on note $\psi^{\prime}$ le morphisme qui se déduite de $\psi$ en remplaçant $(\rho, a, b)$ par $(\rho, a-2, b)$. On suppose qu'il existe une inclusion :

$$
\pi(\psi) \hookrightarrow S p(b, \rho)||^{(a-1) / 2} \times \pi\left(\psi^{\prime}\right) \times\left. S p(b, \rho)\right|^{-(a-1) / 2} .
$$

Alors $\theta_{U}(\psi)$ est par restriction l'action $\theta_{U}\left(\psi^{\prime}\right)$ étendue canoniquement à l'induite de droite.

On montre par exemple (ii) ; on utilise l'inclusion de $\pi\left(\psi^{\prime}\right)$ dans 5.3 (1) (où on remplace $\psi$ par $\left.\psi^{\prime}\right)$ ) ; on remarque que $\psi_{i m p}^{1}=\left(\psi^{\prime}\right)_{i m p}^{1}$ d'où avec l'hypothèse de $(2)$ une inclusion

$$
\begin{aligned}
\pi(\psi) \hookrightarrow & S p(b, \rho)||^{(a-1) / 2} \times\left(\times_{k \geq 1 / 2} \times{ }_{\left(\rho^{\prime}, a^{\prime}, b^{\prime}\right) \in \operatorname{Jord}\left(\psi^{\prime}\right) ;\left(a^{\prime}-1\right) / 2-k \in \mathbb{Z}_{\geq 0}} S p\left(\rho^{\prime}, b^{\prime}\right)||^{k}\right) \times \pi\left(\psi_{i m p}^{1}\right) \\
& \times\left(\times_{k \geq 1 / 2} \times_{\left(\rho^{\prime}, a^{\prime}, b^{\prime}\right) \in \operatorname{Jord}\left(\psi^{\prime}\right) ;\left(a^{\prime}-1\right) / 2-k \in \mathbb{Z}_{\geq 0}} S p\left(b^{\prime}, \rho\right)||^{-k}\right) \times\left. S p(b, \rho)\right|^{-(a-1) / 2} .
\end{aligned}
$$

Cette inclusion ressemble à l'inclusion $5.3(1)$ pour $\pi(\psi)$ à ceci près que $S p(b, \rho)||^{(a-1) / 2}$ et $S p(b, \rho)||^{-(a-1) / 2}$ ne sont pas à leur bonne place. On sait quand même que $\pi(\psi)$ intervient avec multiplicité exactement 1 en tant que sous-quotient à la fois dans (3) et dans 5.3 (1). On construit un entrelacement de (3) vers 5.3 (1), en remplaçant d'abord $S p(b, \rho)^{(a-1) / 2}$ par $\left.S p(b, \rho)\right|^{(a-1) / 2+s}$ et $\left.S p(b, \rho)\right|^{-(a-1) / 2}$ par $S p(b, \rho)||^{-(a-1) / 2-s}$; pour cela on prend un opérateur d'entrelacement standard qui dépend méromorphiquement de $s$. On le note $M(s)$ et on le multiplie par une fonction méromorphe de $s$ de sorte qu'il deviennent holomorphe non nul en $s=0$. On note $M_{0}$ la valeur de cet opérateur en $s=0$. Comme $\pi(\psi)$ est l'unique sous-module irréductible de 5.3 (1), l'image de $M_{0}$ contient $\pi(\psi)$ et envoie donc $\pi(\psi)$ le sous-module de $(3)$ sur $\pi(\psi)$ le sous-module de 5.3 (1). Il est facile de vérifier que $M_{0}$ entrelace l'action de $\theta$ sur (3) qui prolonge canoniquement une action fixée de $\theta \operatorname{sur} \pi\left(\psi_{\text {imp }}^{1}\right)$ en l'action de $\theta$ sur (1) qui prolonge canoniquement cette même action. D'où l'assertion de (ii). 


\subsection{Comparaison des normalisations}

\subsubsection{Le cas où $|\operatorname{Jord}(\psi)|=1$}

Lemme. Supposons que Jord $(\psi)$ a un seul élément $(\rho, a, b)$; dans ce cas $\theta_{W}(\psi)=\theta_{U}(\psi)$.

Si inf $(a, b)=1$ cela résulte des définitions. On démontre l'assertion par récurrence. Supposons donc que inf $(a, b)>1$. On a une inclusion $\pi((\rho, a, b)) \hookrightarrow$

$$
S t(a, \rho)||^{-(b-1) / 2} \times \pi(\rho, a, b-2) \times S t(a, \rho)||^{(b-1) / 2}
$$

qui d'après 5.4 entrelace $\theta_{W}((\rho, a, b))$ et le prolongement canonique de $\theta_{W}((\rho, a, b-2))$. L'hypothèse de récurrence dit que $\theta_{W}((\rho, a, b-2))=\theta_{U}((\rho, a, b-2))$. On a encore une inclusion $: \pi((\rho, a, b-2)) \hookrightarrow$

$$
\left.S p(b-2, \rho)\right|^{(a-1) / 2} \times \pi((\rho, a-2, b-2)) \times S p(b-2, \rho)||^{-(a-1) / 2} .
$$

D'après 5.4 cette inclusion entrelace $\theta_{U}((\rho, a, b-2))$ et le prolongement canonique de $\theta_{U}((\rho, a-2, b-2))$. D'où une inclusion $\pi((\rho, a, b)) \hookrightarrow$

$$
S t(a, \rho)||^{-(b-1) / 2} \times S p(b-2, \rho)||^{(a-1) / 2} \times \pi((\rho, a-2, b-2)) \times S p(b-2, \rho)||^{-(a-1) / 2} \times S t(a, \rho)||^{(b-1) / 2},
$$

qui entrelace $\theta_{W}((\rho, a, b))$ avec le prolongement canonique de $\theta_{U}((\rho, a-2, b-2))$. En travaillant symétriquement, on montre une inclusion $\pi((\rho, a, b)) \hookrightarrow$

$$
S p(b, \rho)||^{(a-1) / 2} \times S t(a-2, \rho)||^{-(b-1) / 2} \times \pi((\rho, a-2, b-2)) \times S t(a-2, \rho)||^{(b-1) / 2} \times S p(b, \rho)||^{-(a-1) / 2}
$$

qui entrelace $\theta_{U}((\rho, a, b))$ avec le prolongement canonique de $\theta_{W}(\rho, a-2, b-2)$; si $(a-2)(b-2)=0$ cette action est l'action triviale. Par récurrence on sait encore que $\theta_{U}((\rho, a-2, b-2))=\theta_{W}((\rho, a-$ $2, b-2))$. On remarque que $S t(a, \rho)^{-(b-1) / 2} \hookrightarrow \rho||^{(a-b) / 2} \times S t(a-2, \rho)^{-(b-1) / 2} \times\left.\rho\right|^{-(a+b) / 2+1}$ et que $S p(b, \rho)||^{(a-1) / 2} \hookrightarrow \rho||^{(a-b) / 2} \times S p(b-2, \rho)||^{(a-1) / 2} \times \rho||^{(a+b) / 2-1}$. L'induite $\left.S t(a-2, \rho)\right|^{-(b-1) / 2}$ est un sous-module de l'induite $\times_{\ell \in[(a-b) / 2-1,-(a+b) / 2+2} \rho||^{\ell}$ tandis que $S p(b-2, \rho)||^{(a-1) / 2}$ est un sous-module de l'induite $\times\left._{\ell \in[(a-b) / 2+1,(a+b) / 2-2]} \rho\right|^{\ell}$. D'où les isomorphismes (cf. la preuve de 1.12) :

$$
\begin{aligned}
\rho||^{(a+b) / 2-1} \times S t(a-2, \rho)||^{-(b-1) / 2} & \simeq S t(a-2, \rho)||^{-(b-1) / 2} \times \rho||^{(a+b) / 2-1} ; \\
\rho||^{-(a+b) / 2+1} \times S p(b-2, \rho)||^{(a-1) / 2} & \simeq S p(b-2, \rho)||^{(a-1) / 2} \times \rho||^{-(a+b) / 2+1} ; \\
S t(a-2, \rho)^{-(b-1) / 2} \times S p(b-2, \rho)||^{(a-1) / 2} & \simeq S p(b-2, \rho)||^{(a-1) / 2} \times S t(a-2, \rho)^{-(b-1) / 2} .
\end{aligned}
$$

On peut donc remplacer (5) par l'inclusion $\pi((\rho, a, b)) \hookrightarrow$

$$
\begin{gathered}
\rho \|\left.\right|^{(a-b) / 2} \times S p(b-2, \rho)||^{(a-1) / 2} \times S t(a-2, \rho)||^{-(b-1) / 2} \times \rho||^{(a+b) / 2-1} \times \pi((\rho, a-2, b-2)) \\
\times\left.\rho\left\|^{-(a+b) / 2+1} \times S t(a-2, \rho)\right\|\right|^{(b-1) / 2} \times\left. S p(b-2, \rho)\left\|\left.\right|^{-(a-1) / 2} \times \rho\right\|\right|^{-(a-b) / 2}
\end{gathered}
$$

et remplacer (4) par l'inclusion $\pi((\rho, a, b)) \hookrightarrow$

$$
\begin{aligned}
\rho||^{(a-b) / 2} & \times S t(a-2, \rho)||^{(b-1) / 2} \times S p(b-2, \rho)||^{-(a-1) / 2} \times \rho||^{-(a+b) / 2+1} \times \pi((\rho, a-2, b-2)) \\
& \times \rho||^{(a+b) / 2-1} \times S p(b-2, \rho)||^{(b-1) / 2} \times S t(a-2, \rho)||^{-(a-1) / 2} \times \rho||^{-(a-b) / 2} .
\end{aligned}
$$

Et ces inclusions entrelacent pour $(4)^{\prime}, \theta_{W}((\rho, a, b))$ avec le prolongement canonique de $\theta((\rho, a-2, b-2))$ et pour $(5)^{\prime}, \theta_{U}((\rho, a, b))$ avec le prolongement canonique de $\theta((\rho, a-2, b-2))$. On peut encore échanger dans $(4)^{\prime} S t(a-2, \rho)||^{-(b-1) / 2} \times\left. S p(b-2, \rho)\right|^{(a-1) / 2}$ en $S p(b-2, \rho)||^{(a-1) / 2} \times S t(a-2, \rho)||^{-(b-1) / 2}$ et dualement $\left.S p(b, \rho)\right|^{-(a-1) / 2} \times\left. S t(a-2, \rho)\right|^{(b-1) / 2}$ en $S t(a-2, \rho)||^{(b-1) / 2} \times S p(b-2, \rho)||^{-(a-1) / 2}$ puis remarquer que $\rho||^{(a+b) / 2-1} \times \pi((\rho, a-2, b-2)) \times\left.\rho\right|^{-(a+b) / 2+1}$ est irréductible; cela résulte de ce que les représentations $\rho||^{\ell}$ dans le support cuspidal de $\pi((\rho, a-2, b-2))$ vérifient $(a+b) / 2-1-\ell>1$. On peut donc encore échanger et transformer le deuxième membre de $(4)^{\prime}$ en celui de $(5)^{\prime}$ par des entrelacements qui entrelacent les prolongements canoniques de $\theta((\rho, a-2, b-2))$ (cf. 1.9). Pour pouvoir conclure que 
$\theta_{W}((\rho, a, b))=\theta_{U}((\rho, a, b))$, il faut encore remarquer que $(5)^{\prime}$ admet $\pi((\rho, a, b))$ comme sous-module irréductible avec multiplicité 1 ; supposons qu'il existe une inclusion de $\Pi:=\pi((\rho, a, b)) \oplus \pi((\rho, a, b))$ dans le deuxième membre de $(5)^{\prime}$. On reprend les arguments en sens inverse; on écrit une suite exacte :

$$
\begin{gathered}
0 \rightarrow S p(b, \rho)||^{(a-1) / 2} \times S t(a-2, \rho)||^{-(b-1) / 2} \rightarrow \\
\rho||^{(a-b) / 2} \times S p(b-2, \rho)||^{(a-1) / 2} \times S t(a-2, \rho)||^{-(b-1) / 2} \times \rho||^{(a+b) / 2-1} \\
\rightarrow \tau \rightarrow 0,
\end{gathered}
$$

ce qui définit $\tau$. On vérifie qu'il existe $x \neq(a-b) / 2$ tel que $J a c_{x} \tau \neq 0$. On considère l'image de $\Pi$ dans

$$
\tau \times \pi((\rho, a-2, b-2)) \times \rho||^{-(a+b) / 2+1} \times S t(a-2, \rho)||^{(b-1) / 2} \times S p(b-2, \rho)||^{-(a-1) / 2} \times \rho||^{-(a-b) / 2} .
$$

Si elle était non nulle, pour un $x$ comme ci-dessus, on aurait $J a c_{x} \pi((\rho, a, b)) \neq 0$, ce qui est impossible. On montre de façon analogue que $\Pi$ doit être inclus dans

$S p(b, \rho)||^{(a-1) / 2} \times S t(a-2, \rho)||^{-(b-1) / 2} \times \pi((\rho, a-2, b-2)) \times\left. S t(a-2, \rho)\right|^{(b-1) / 2} \times S p(b, \rho)||^{-(a-1) / 2}$.

Un calcul de module de Jacquet maintenant immédiat montre que cette induite n'a qu'un unique sous-module irréductible. D'où a fortiori notre assertion qui termine la preuve.

\subsubsection{Action de $\theta$ et opérateurs d'entrelacement normalisés 1.}

Pour pouvoir comparer les actions $\theta_{W}(\psi)$ et $\theta_{U}(\psi)$ on va les comparer à des actions obtenues avec des opérateurs d'entrelacement normalisés et on va montrer que le signe dont diffèrent ces 2 actions est un quotient de facteurs de normalisation.

D’une façon très générale, soit $\delta, \delta^{\prime}$ des représentations $\theta$-invariantes; on considère un opérateur d'entrelacement, pour $s \in \mathbb{C}$ voisin de 0 :

$$
N(s): \delta||^{s} \times \delta^{\prime} \rightarrow \delta^{\prime} \times \delta||^{s}
$$

dépendant méromorphiquement de $s$; exactement on note $M(s)$ l'opérateur d'entrelacement standard (cf. 1.6) et on suppose qu'il existe une fonction méromorphe de $s, r(s)$ tel que $N(s)$ soit l'opérateur $r(s) M(s)$. On suppose que $N(s)$ est holomorphe en $s=0$. On suppose aussi que $\delta$ et $\delta^{\prime}$ sont munis d'une action de $\theta$, notée $\theta_{\delta}$ et $\theta_{\delta^{\prime}}$. On peut alors définir une action de $\theta$ sur l'induite $\delta \times \delta^{\prime}$, en posant pour toute section $f$ à valeurs dans $\delta \otimes \delta^{\prime}$ et pour tout élément $g$ du groupe :

$$
\theta . f(g):=\left(\theta_{\delta} \otimes \theta_{\delta^{\prime}} \circ i n v\right)(N(0) f)(\theta(g))=:(\Theta \circ N(0)) f
$$

où inv est l'échange $\delta^{\prime} \otimes \delta$ dans $\delta \otimes \delta^{\prime}$. Pour que $\theta^{2} . f$ soit égal à 1 , il faut que l'on ait

$$
((\Theta \circ N(-s) \circ \Theta) \circ N(s))_{s=0}=1 .
$$

Pour $\operatorname{Re}(-s)>>0$, on vérifie que pour l'opérateur d'entrelacement standard $\Theta \circ M(-s) \circ \Theta$ est l'opérateur d'entrelacement standard :

$$
M^{\prime}(-s): \quad \delta^{\prime} \times \delta||^{s} \rightarrow \delta||^{s} \times \delta^{\prime}
$$

cela se fait en écrivant explicitement l'intégrale. La condition devient donc

$$
\left(r(s) r(-s) M^{\prime}(-s) M(s)\right)_{s=0}=1 .
$$

Quand on représente l'induite $\delta||^{s} \times \delta^{\prime}$ dans un espace indépendant de $s$, le produit $M^{\prime}(-s) \circ M(s)$ est une homothétie avec un coefficient qui est une fonction méromorphe de $s, c(s):=M^{\prime}(-s) \circ M(s)$.Pour 
avoir une action de $\theta$ avec la définition ci-dessus, suffit donc que $r(s) r(-s)=c(s)^{-1}$. Des choix de $r(s)$, plusieurs sont possibles; on considère d'abord celui suggéré par Langlands et calculé explicitement par Shahidi [23], à ceci près que l'on modifiera les facteurs $\epsilon$. On pose donc :

$$
N_{L}(s):=E\left(\delta, \delta^{\prime}, s\right) L\left(\delta \times \delta^{\prime}, s\right) / L\left(\delta \times \delta^{\prime}, 1+s\right),
$$

où $E(s):=\prod_{\tau, \tau^{\prime}} \epsilon\left(\tau \times \tau^{\prime}, s\right)$; dans ce produit $\tau$ parcourt l'ensemble des représentations cuspidales formant le support cuspidale de $\delta$ et $\tau^{\prime}$ parcourt l'ensemble analogue pour $\delta^{\prime}$.

On applique cela à $\pi(\psi)$ de la façon suivante : on fixe $(\rho, a, b) \in \operatorname{Jord}(\psi)$ tel que $b$ soit maximum. On note $\psi^{\prime}$ le morphisme qui se déduit de $\psi$ en enlevant ce triplet; on a déjà défini $\pi((\rho, a, b))$ et on a l'isomorphisme :

$$
\pi(\psi)=\pi((\rho, a, b)) \times \pi\left(\psi^{\prime}\right) .
$$

On a démontré en [21] un certain nombre de propriétés pour les opérateurs d'entrelacement normalisés à la Langlands-Shahidi :

$$
N_{L}(s): \pi(\rho, a, b)||^{s} \times \pi\left(\psi^{\prime}\right) \rightarrow \pi\left(\psi^{\prime}\right) \times \pi(\rho, a, b)||^{s} .
$$

La première propriété est une formule de produit; par exemple quand on inclut $\pi(\rho, a, b)$ dans $S t(a, \rho)^{(b-1) / 2} \times \pi(\rho, a-2, b-2) \times S t(a, \rho)^{-(b-1) / 2}, N_{L}(s)$ est un produit, ici, de 3 opérateurs d'entrelacements normalisés à la Langlands-Shahidi. C'est facile car cela résulte uniquement la formule de produit évidente pour les facteurs de normalisations introduits. On utilisera aussi la formule de produit suivant la décomposition $\pi\left(\psi^{\prime}\right)=\times_{\left(\rho^{\prime}, a^{\prime}, b^{\prime}\right) \in \operatorname{Jord}\left(\psi^{\prime}\right)} \pi\left(\rho^{\prime}, a^{\prime}, b^{\prime}\right)$.

La deuxième propriété est nettement plus difficile; on dit en suivant [21] que deux représentations $\pi(\rho, a, b)||^{s}$ (s réel) et $\pi\left(\rho^{\prime}, a^{\prime}, b^{\prime}\right)$ sont liées si $\rho=\rho^{\prime}$ et

$$
\left|\left(a-a^{\prime}\right) / 2\right|+\left|\left(b-b^{\prime}\right) / 2\right|<|s| \leq\left(a+a^{\prime}+b+b^{\prime}\right) / 2-1 .
$$

C'est une généralisation de la notion de segments liés introduite par Zelevinsky, condition qui est naturelle quand on travaille avec les composantes locales des formes automorphes de carré intégrables résiduelles des groupes $G L$.

Et la propriété est que l'opérateur d'entrelacement :

$$
N_{L}\left((\rho, a, b),\left(\rho^{\prime}, a^{\prime}, b^{\prime}\right), s\right): \pi(\rho, a, b)||^{s} \times \pi\left(\rho^{\prime}, a^{\prime}, b^{\prime}\right) \rightarrow \pi\left(\rho^{\prime}, a^{\prime}, b^{\prime}\right) \times\left.\pi(\rho, a, b)\right|^{s}
$$

est holomorphe en tout point $s$ réel tel que $\pi(\rho, a, b)||^{s}$ et $\pi\left(\rho^{\prime}, a^{\prime}, b^{\prime}\right)$ ne sont pas liés.

On admet que l'on sait définir par récurrence une action $\theta_{L}\left(\psi^{\prime}\right)$ sur $\pi\left(\psi^{\prime}\right)$ et on pose

$$
\theta_{L}(\psi):=\left(\left(\theta(\rho, a, b) \otimes \theta_{L}\left(\psi^{\prime}\right)\right) \circ i n v\right) \circ N_{L}(0) .
$$

La propriété de multiplicativité des opérateurs d'entrelacement normalisés montre que $\theta_{L}(\psi)$ est indépendant du choix de $(\rho, a, b) \in \operatorname{Jord}(\psi)$.

On suppose ici que $b \geq 2$ et on construit les morphismes suivants, les inclusions sont naturelles et les flèches sont les opérateurs d'entrelacement normalisés à la Langlands-Shahidi :

$$
\begin{gathered}
\pi(\rho, a, b)||^{s} \times \pi\left(\psi^{\prime}\right) \hookrightarrow S t(a, \rho)^{-(b-1) / 2+s} \times \pi(\rho, a, b-1)||^{1 / 2+s} \times \pi\left(\psi^{\prime}\right) \rightarrow \\
S t(a, \rho)||^{-(b-1) / 2+s} \times \pi\left(\psi^{\prime}\right) \times \pi(\rho, a, b-1)||^{s+1 / 2} \hookrightarrow \\
S t(a, \rho)||^{-(b-1) / 2+s} \times \pi\left(\psi^{\prime}\right) \times \pi(\rho, a, b-2)||^{s} \times S t(a, \rho)||^{(b-1) / 2+s} \\
\rightarrow S t(a, \rho)||^{-(b-1) / 2+s} \times \pi(\rho, a, b-2)||^{s} \times \pi\left(\psi^{\prime}\right) \times S t(a, \rho)||^{(b-1) / 2+s} .
\end{gathered}
$$

L'opérateur d'entrelacement défini entre (2) et (3) est holormophe en $s=0$ car pour tout $\left(\rho, a^{\prime}, b^{\prime}\right) \in$ $\operatorname{Jord}\left(\psi^{\prime}\right),\left|\left(a-a^{\prime}\right) / 2\right|+\left|\left(b-1-b^{\prime}\right) / 2\right| \geq 1 / 2$ sauf a priori si $a=a^{\prime}$ et $b^{\prime}=b+1$ mais la différence de parité suffit alors à l'holomorphie. L'opérateur qui passe de (2) à (3) est aussi holomorphe en $s=0$; on construit donc une application avec des opérateurs d'entrelacement normalisés

$$
n_{L}(0): \pi(\rho, a, b) \times \pi\left(\psi^{\prime}\right) \rightarrow S t(a, \rho)^{-(b-1) / 2} \times \pi(\rho, a, b-2) \times \pi\left(\psi^{\prime}\right) \times S t(a, \rho)^{(b-1) / 2}
$$


cette application est certainement non nulle car tous les opérateurs d'entrelacement utilisés sont inversibles (condition de non liaison qui ne dépend que de $|s|$ ).

Lemme. L'opérateur $n_{L}(0)$ entrelace $\theta_{L}(\psi)$ avec le prolongement canonique de $\theta_{L}\left(\psi^{\prime},(\rho, a, b-2)\right)$.

En tant qu'opérateur méromorphe $n_{L}(s)$ coïncide avec l'opérateur d'entrelacement normalisé à la Langlands-Shahidi :

$$
\begin{gathered}
\pi(\rho, a, b)||^{s} \times \pi\left(\psi^{\prime}\right) \hookrightarrow S t(a, \rho)||^{(b-1) / 2+s} \times \pi(\rho, a, b-2)||^{s} \times S t(a, \rho)||^{(b-1) / 2} \times \pi\left(\psi^{\prime}\right) \rightarrow \\
S t(a, \rho)||^{-(b-1) / 2+s} \times \pi(\rho, a, b-2)||^{s} \times \pi\left(\psi^{\prime}\right) \times S t(a, \rho)||^{(b-1) / 2+s}
\end{gathered}
$$

On note $\theta_{L}^{+}\left(\psi^{\prime},(\rho, a, b-2)\right)$ le prolongement canonique de $\theta_{L}\left(\psi^{\prime},(\rho, a, b-2)\right.$; il s'obtient comme la valeur en $s=0$ du composé de l'opérateur d'entrelacement normalisé qui échange les 2 facteurs de $\left.\pi(\rho, a, b-2)\right|^{s} \times \pi\left(\psi^{\prime}\right)$ composé avec l'action naturelle de $\theta$ sur l'induite

$$
\begin{gathered}
S t(a, \rho)||^{-(b-1) / 2+s} \times \pi\left(\psi^{\prime}\right) \times \pi(\rho, a, b-2)||^{s} \times S t(a, \rho)||^{(b-1) / 2+s} \rightarrow \\
S t(a, \rho)||^{-(b-1) / 2-s} \times \pi(\rho, a, b-2)||^{s} \times \pi\left(\psi^{\prime}\right) \times S t(a, \rho)||^{(b-1) / 2-s} .
\end{gathered}
$$

On note $\theta_{L}^{+}\left(\psi^{\prime},(\rho, a, b-2), s\right)$ cet opérateur.

Pour appliquer d'abord $\theta_{L}(\psi)$, il faut réaliser $\theta_{L}(\psi)$ comme la valeur en $s=0$ de l'action naturelle de $\theta: \pi(\rho, a, b)||^{s} \times \pi\left(\psi^{\prime}\right)$ dans $\pi\left(\psi^{\prime}\right) \times \pi(\rho, a, b)^{-s}$ composée avec l'opérateur d'entrelacement normalisé de $\pi\left(\psi^{\prime}\right) \times \pi(\rho, a, b)^{-s}$ dans $\pi(\rho, a, b)||^{-s} \times \pi\left(\psi^{\prime}\right)$; opérateur que l'on note $\theta_{L}(\psi, s)$. Ensuite on applique $n_{L}(-s)$ et on montre alors facilement l'égalité d'opérateurs méromorphes :

$$
\theta_{L}^{+}\left(\psi^{\prime},(\rho, a, b-2), s\right) \circ n_{L}(s)=n_{L}(-s) \circ \theta_{L}(\psi, s) .
$$

En faisant $s=0$, on obtient l'assertion.

Corollaire. L'inclusion de Langlands de 5.2 (1) entrelace $\theta_{L}(\psi)$ et le prolongement canonique de $\theta_{L}\left(\psi_{i m p}^{2}\right)$.

C'est un corollaire immédiat. Remarquons qu'il n'y a pas d'espoir d'avoir une égalité entre $\theta_{L}(\psi)$ et $\theta_{W}(\psi)$ car $\theta_{L}(\psi)$ dépend de $E(s)$ (cf ci-dessus) qu'on a fixé sans précaution. Précisément, il semble que l'on puisse toujours très raisonablement remplacer $N_{L}(s)$ par $-N_{L}(s)$ ce qui évidemment change $\theta_{L}$ en son opposé. On cherche à calculer $\theta_{W}(\psi) / \theta_{U}(\psi)$ et on va le réaliser à l'aide de quotients de facteurs de normalisation où $E(s)$ ne jouera plus de rôle; les seuls termes qui compteront seront les fonctions $L$ qui ne dépendent d'aucun choix.

\subsubsection{Action de $\theta$ et opérateurs d'entrelacement normalisés 2 .}

Ce que l'on a fait ci-dessus avec la normalisation de Langlands-Shahidi peut se faire en remplaçant les représentations de Steinberg par les représentations de Speh. On va le faire explicitement. Rappelons la valeur du facteur de normalisation pour l'entrelacement entre 2 blocs :

$$
N_{L}(s): \quad \pi(\rho, a, b)^{s} \times \pi\left(\rho^{\prime}, a^{\prime}, b^{\prime}\right) \rightarrow \pi\left(\rho^{\prime}, a^{\prime}, b^{\prime}\right) \times \pi(\rho, a, b)||^{s} .
$$

On n'a pas besoin de la valeur de $E(s)$ introduite dans 5.5.2, pour $r_{L}(s)$ tel que $r_{L}(s) N_{L}(s)$ soit l'opérateur d'entrelacement standard :

$$
\begin{gathered}
r_{L}(s)=E(s) \underset{\substack{j \in[-(b-1) / 2,(b-1) / 2], j^{\prime} \in\left[-\left(b^{\prime}-1\right) / 2,\left(b^{\prime}-1\right) / 2\right]}}{ } L\left(\rho \times \rho^{\prime}, j+j^{\prime}+\left|\left(a-a^{\prime}\right) / 2\right|+s\right) / L\left(\rho \times \rho^{\prime}, j+j^{\prime}+\left(a+a^{\prime}\right) / 2+s\right) .
\end{gathered}
$$


On pose, a priori, pour le même $E(s)$ :

$$
\begin{gathered}
r_{S}(s):=E(s) \underset{\substack{i \in[(a-1) / 2,-(a-1) / 2], i^{\prime} \in\left[\left(a^{\prime}-1\right) / 2,-\left(a^{\prime}-1\right) / 2\right]}}{ } L\left(\rho \times \rho^{\prime}, i+i^{\prime}+\left|\left(b-b^{\prime}\right) / 2\right|-s\right) / L\left(\rho \times \rho^{\prime}, i+i^{\prime}+\left(b+b^{\prime}\right) / 2-s\right) .
\end{gathered}
$$

Cela permet de poser $N_{S}(s):=r_{S}(s)^{-1} M(s)$, où $M(s)$ est encore l'opérateur d'entrelacement normalisé. Et on généralise à plusieurs blocs en composant les opérateurs.

Remarque. Pour $\rho$ et $\rho^{\prime}$ des représentations cuspidales autoduales irréductibles, le quotient $r_{L}(s) / r_{S}(s)$ est holormophe en $s=0$; en ce point il vaut 1 si $\rho \neq \rho^{\prime}$ et vaut le signe $(-1)^{\text {inf }\left(a, a^{\prime}\right) \inf \left(b, b^{\prime}\right)}$ si $\rho \simeq \rho^{\prime}$. Plus généralement ce quotient est holomorphe en tout point $s$ tel que les représentations $\left.\pi(\rho, a, b)\right|^{s}$ et $\pi\left(\rho^{\prime}, a^{\prime}, b^{\prime}\right)$ ne sont pas liées.

On pose $a_{+}=\sup \left(a, a^{\prime}\right), a_{-}=\inf \left(a, a^{\prime}\right), b_{+}=\sup \left(b, b^{\prime}\right), b_{-}=\inf \left(b, b^{\prime}\right)$. On récrit

$$
\begin{aligned}
& \prod_{j \in[-(b-1) / 2,(b-1) / 2], j^{\prime} \in\left[-\left(b^{\prime}-1\right) / 2,\left(b^{\prime}-1\right) / 2\right]} \frac{L\left(\rho \times \rho^{\prime}, j+j^{\prime}+\left|\left(a-a^{\prime}\right) / 2\right|+s\right)}{L\left(\rho \times \rho^{\prime}, j+j^{\prime}+\left(a+a^{\prime}\right) / 2+s\right)}= \\
& \Pi \\
& k^{\prime} \in\left[-\left(b_{+}-1\right) / 2,\left(b_{+}-1\right) / 2\right] \\
& \frac{L\left(\rho \times \rho^{\prime}, k+k^{\prime}+\left(a_{+}-1\right) / 2+\ell+s\right)}{L\left(\rho \times \rho^{\prime}, k+k^{\prime}+\left(a_{+}-1\right) / 2+\ell+1+s\right)}= \\
& k \in\left[-\left(b_{-}-1\right) / 2,\left(b_{-}-1\right) / 2\right] \text {; } \\
& \ell \in\left[\left(a_{-}-1\right) / 2,-\left(a_{-}-1\right) / 2\right] \\
& =\prod_{k \in\left[\left(b_{-}-1\right) / 2,-\left(b_{-}-1\right) / 2\right],} \frac{L\left(\rho \times \rho^{\prime}, k+\ell+\left(a_{+}-1\right) / 2-\left(b_{+}-1\right) / 2+s\right)}{L\left(\rho \times \rho^{\prime}, k+\ell+\left(a_{+}-1\right) / 2+\left(b_{+}-1\right) / 2+1+s\right)} . \\
& \ell \in\left[\left(a_{-}-1\right) / 2,-\left(a_{-}-1\right) / 2\right]
\end{aligned}
$$

De façon analogue, on obtient :

$$
\begin{aligned}
& \prod_{\substack{i \in[(a-1) / 2,-(a-1) / 2] \\
i^{\prime} \in\left[\left(a^{\prime}-1\right) / 2,-\left(a^{\prime}-1\right) / 2\right]}} \frac{L\left(\rho \times \rho^{\prime}, i+i^{\prime}+\left|\left(b-b^{\prime}\right) / 2\right|-s\right)}{L\left(\rho \times \rho^{\prime}, i+i^{\prime}+\left(b+b^{\prime}\right) / 2-s\right)}= \\
& \prod_{\substack{k \in\left[\left(b_{-}-1\right) / 2,-\left(b_{-}-1\right) / 2\right] \\
\ell \in\left[\left(a_{-}-1\right) / 2,-\left(a_{-}-1\right) / 2\right]}}^{\frac{L\left(\rho \times \rho^{\prime}, k+\ell+\left(b_{+}-1\right) / 2-\left(a_{+}-1\right) / 2-s\right)}{L\left(\rho \times \rho^{\prime}, k+\ell+\left(a_{+}-1\right) / 2+\left(b_{+}-1\right) / 2+1-s\right)} .}
\end{aligned}
$$

Le seul cas où les fonctions $L$ ne valent pas identiquement 1 et celui où $\rho^{\prime}=\chi \otimes \rho$ avec $\chi$ est un caractère non ramifié de $F^{*}$, y compris, évidemment le caratère trivial. En particulier $\rho$ et $\rho^{\prime}$ sont des représentations d'un même groupe $G L(d, F)$.

Pour ce cas, on revient à la formule explicite pour $L\left(\rho \times \rho^{\prime}, s\right)$ donnée par Shahidi ??; le produit porte sur tous les caractères non ramifiés $\tau$ de $F^{*}$ vus comme caractères de $G L(d, F)$ via le déterminant tels que $\rho \simeq \tau \otimes \rho^{\prime}$ et $\omega$ est une uniformisante :

$$
L\left(\rho \times \rho^{\prime}, s\right)=\prod_{\tau}\left(1-\tau(\omega) q^{-s}\right)^{-1} .
$$

On note $M$ l'ordre du groupe des caracères non ramifiés, $\tau^{\prime}$ tel que $\rho \simeq \tau^{\prime} \otimes \rho$ et on a encore : $L\left(\rho \times \rho^{\prime}, s\right)=\left(1-\zeta_{\rho, \rho^{\prime}} q^{-M s}\right)$ où $\zeta_{\rho, \rho^{\prime}}=+1$ si $\rho=\rho^{\prime}$ et -1 sinon.

Or pour tout $k, \ell$

$$
L\left(\rho \times \rho^{\prime}, k+\ell+\left(b_{+}-1\right) / 2-\left(a_{+}-1\right) / 2-s\right)=-\zeta_{\rho, \rho^{\prime}} q^{M s} \chi\left(L\left(\rho \times \rho^{\prime},-k-\ell+\left(a_{+}-1\right) / 2-\left(b_{+}-1\right) / 2+s\right)\right.
$$


pour $M$ convenable (ne dépendant d'ailleurs que de $\rho$ ). On remarque que le produit porte sur des ensembles symétriques par rapport à 0 . Ainsi, pour $m$ convenable

$$
r_{L}(s) / r_{S}(s)=\left(-\zeta_{\rho, \rho^{\prime}}\right)^{a_{-} b_{-}} q^{m s} \prod_{k, \ell} L\left(\rho \times \rho^{\prime}, k+\ell+\left(a_{+}+b_{+}\right) / 2-s\right) / L\left(\rho \times \rho^{\prime}, k+\ell+\left(a_{+}+b_{+}\right) / 2+s\right),
$$

où $k, \ell$ varient comme ci-dessus. Il suffit de démontrer qu'en $s=0$ et plus généralement en tout point $s$ satisfaisant :

$$
|s| \leq\left(a_{+}-a_{-}\right) / 2+\left(b_{+}-b_{-}\right) / 2 \text { ou }\left(a_{+}+a_{-}+b_{+}+b_{-}\right) / 2-1<|s|
$$

numérateur et dénominateur sont holomorphes. Or on a, pour tout $k, \ell$ comme ci-dessus :

$$
\begin{gathered}
k+\ell+\left(a_{+}+b_{+}\right) / 2 \leq\left(b_{-}-1\right) / 2+\left(a_{-}-1\right) / 2+\left(a_{+}+b_{+}\right) / 2=\left(a_{+}+a_{-}+b_{+}+b_{-}\right) / 2-1 \\
k+\ell+\left(a_{+}+b_{+}\right) / 2 \geq-\left(b_{-}-1\right) / 2-\left(a_{-}-1\right) / 2+\left(a_{+}-1\right) / 2+\left(b_{+}+1\right) / 2+1= \\
\left(a_{+}-a_{-}\right) / 2+\left(b_{+}-b_{-}\right) / 2+1>\left(a_{+}-a_{-}\right) / 2+\left(b_{+}-b_{-}\right) / 2 .
\end{gathered}
$$

Cela prouve l'holomorphie pour les valeurs de $s$ fixées, puisque $L\left(\rho \times \rho^{\prime}, s^{\prime}\right)$ est holomorphe non nul en tout $s^{\prime}$ réel tel que $s^{\prime} \neq 0$.

Corollaire. Les opérateurs $N_{S}(s)$ ont les mêmes propriétés de multiplicativité et d'holomorphie que les opérateurs $N_{L}(s)$ quand on remplace Steinberg par Speh .

La première assertion résulte de la construction même et la deuxième du lemme ci-dessus.

On définit alors $\theta_{S}(\psi)$ comme on a défini $\theta_{L}(\psi)$ en remplaçant $N_{L}(s)$ par $N_{S}(s)$ et on a le corollaire :

Corollaire. L'inclusion de $\pi(\psi)$ dans 5.3 (1) entrelace $\theta_{S}(\psi)$ avec le prolongement canonique de $\theta_{S}\left(\psi_{i m p}^{1}\right)$.

\subsubsection{Calcul de $\theta_{W}(\psi) / \theta_{U}(\psi)$.}

On a défini les facteurs de normalisations $r_{L}(s), r_{S}(s)$; ils dépendent de $\psi$ et ici on met $\psi$ dans la définition. Et on pose $a(\psi):=\left(r_{L}(\psi)(s) / r_{S}(\psi)(s)\right)_{s=0}$. Pour éviter toute ambiguïté, on précise que quand on somme sur les couples de triplets $\left((\rho, a, b),\left(\stackrel{s=0}{\rho^{\prime}}, a^{\prime}, b^{\prime}\right)\right) \in \operatorname{Jord}(\psi)$, on regarde ces couples avec leur ordre, c'est-à-dire qu'un tel couple est différent de $\left(\left(\rho^{\prime}, a^{\prime}, b^{\prime}\right),(\rho, a, b)\right)$ mais on supposera toujours $(\rho, a, b)$ est différent de $\left(\rho, a^{\prime}, b^{\prime}\right)$; c'est pour cela que beaucoup de somme sont affectées du coefficient $1 / 2$. Il y a encore une autre ambiguïté qui vient du fait que $\operatorname{Jord}(\psi)$ peut avoir de la multiplicité; il faut en tenir compte, c'est-à-dire qu'un couple $\left((\rho, a, b),\left(\rho^{\prime}, a^{\prime}, b^{\prime}\right)\right)$ vient dans les formules avec la multiplicité produit de la multiplicité de $(\rho, a, b)$ dans $\operatorname{Jord}(\psi)$ avec la multiplicité de $\left(\rho^{\prime}, a^{\prime}, b^{\prime}\right)$ dans $\operatorname{Jord}(\psi)$.

Théorème. L'action $\theta_{W}(\psi)$ diffère de $\theta_{U}(\psi)$ par le signe $a(\psi) a\left(\psi_{i m p}^{1}\right) a\left(\psi_{i m p}^{2}\right) a\left(\psi_{\text {imp }, i m p}\right)$. Ex-

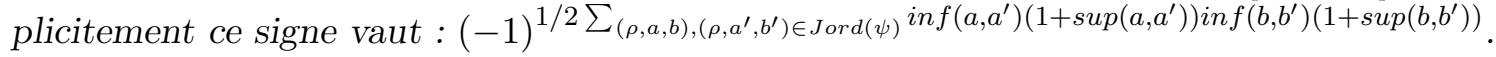

On reprend 5.2 (5); pour des bonnes représentations $\sigma, \tau$, où $\sigma$ est un produit de représentations de Steinberg tordues par des caractères négatifs et $\tau$ un produit de cuspidales tordues par des caractères positifs et que l'on voit donc comme des représentations de Speh tordues par des caractères positifs, on a des inclusions :

$$
\pi(\psi) \hookrightarrow \sigma \times \pi\left(\psi_{i m p}^{2}\right) \times{ }^{\theta} \sigma \hookrightarrow \sigma \times \tau \times \pi\left(\psi_{i m p, i m p}\right) \times{ }^{\theta} \tau \times{ }^{\theta} \sigma .
$$


L'action $\theta_{W}(\psi)$ est la restriction à $\psi$ du prolongement canonique de l'action de $\theta$ sur $\pi\left(\psi_{\text {imp,imp }}\right)$ qui agit naturellement sur l'espace des fonctionnelles de Whittaker pour le caractère additif fixé. On note $\theta_{f}$ cette action de $\theta$ sur $\pi\left(\psi_{i m p, i m p}\right)$.

La dernière inclusion entrelace le prolongement canonique de $\theta_{S}\left(\psi_{i m p}^{2}\right)$ avec le prolongement canonique de $\theta_{S}\left(\psi_{i m p, i m p}\right)$ tandis que la première inclusion entrelace le prolongement canonique de $\theta_{L}\left(\psi_{i m p}^{2}\right)$ avec $\theta_{L}(\psi)$. Posons $c_{2}:=\theta_{f} / \theta_{S}\left(\psi_{i m p, i m p}\right)$. Ainsi le prolongement canonique de $\theta_{f}$ se restreint en $c_{2}$ fois le prolongement canonique de $\theta_{S}\left(\psi_{i m p}^{2}\right)$ sur l'induite du milieu et donc par définition de $a\left(\psi_{\text {imp }}^{2}\right)$ en $c_{2} a\left(\psi_{\text {imp }}^{2}\right)$ fois le prolongement canonique de $\theta_{L}\left(\psi_{\text {imp }}^{2}\right)$. Ainsi $\theta_{W}(\psi)$ diffère de $\theta_{L}(\psi)$ par le signe $c_{2} a\left(\psi_{i m p}^{2}\right)$. On procède de façon analogue avec les normalisations unipotentes. On pose $c_{1}$ le signe $\theta_{f} / \theta_{L}\left(\psi_{i m p, i m p}\right)$ et on voit que $\theta_{U}(\psi)$ diffère de $\theta_{S}(\psi)$ par le signe $c_{1} a\left(\psi_{i m p}^{1}\right)$. Par définition $c_{1} c_{2}=a\left(\psi_{i m p, i m p}\right)$ et on obtient donc :

$$
\theta_{W}(\psi) / \theta_{U}(\psi)=c_{2} a\left(\psi_{i m p}^{2}\right) c_{1} a\left(\psi_{i m p}^{1}\right) \theta_{L}(\psi) / \theta_{S}(\psi)=a\left(\psi_{i m p, i m p}\right) a\left(\psi_{i m p}^{1}\right) a\left(\psi_{i m p}^{2}\right) a(\psi) .
$$

C'est la première assertion du théorème. Passons au calcul explicite. On rappelle que

$$
a(\psi)=(-1)^{\sum_{(\rho, a, b),\left(\rho, a^{\prime}, b^{\prime}\right) \in \operatorname{Jord}(\psi)} \inf \left(a, a^{\prime}\right) \inf \left(b, b^{\prime}\right)} .
$$

On a un calcul analogue pour les autres $a(?)$; pour $a\left(\psi_{i m p}^{1}\right)$ il faut considérer $\operatorname{Jord}\left(\psi_{i m p}^{1}\right)$ c'est-à-dire uniquement les couples $(\rho, a, b),\left(\rho, a^{\prime}, b^{\prime}\right)$ tel que inf $\left(a, a^{\prime}\right)$ et $\sup \left(a, a^{\prime}\right)$ sont impairs et la contribution d'un tel couple est $(-1)^{\text {inf }\left(b, b^{\prime}\right)}$. Pour considérer tous les couples, il suffit de prendre comme contribution $(-1)^{\text {inf }\left(a, a^{\prime}\right) \sup \left(a, a^{\prime}\right) \inf \left(b, b^{\prime}\right)}$. On procéde de façon analogue pour $\psi_{i m p}^{2} ; \psi_{\text {imp }, \text { imp }}$ la contribution ne vient que des couples $(\rho, a, b),\left(\rho, a^{\prime}, b^{\prime}\right)$ tel que $a, a^{\prime}, b, b^{\prime}$ sont impairs et la contribution est $(-1)$, on peut donc l'écrire, en considérant tous les couples, sous la forme $(-1)^{\inf \left(a, a^{\prime}\right) \sup \left(a, a^{\prime}\right) \inf \left(b, b^{\prime}\right) \sup \left(b, b^{\prime}\right)}$, on trouve le signe $(-1)^{x}$ où

$$
\begin{aligned}
x & =\sum_{(\rho, a, b),\left(\rho, a^{\prime}, b^{\prime}\right) \in \operatorname{Jord}(\psi)} \inf \left(a, a^{\prime}\right) \inf \left(b, b^{\prime}\right)+\inf \left(a, a^{\prime}\right) \inf \left(b, b^{\prime}\right) \sup \left(b, b^{\prime}\right)+ \\
& +\inf \left(b, b^{\prime}\right) \inf \left(a, a^{\prime}\right) \sup \left(b, b^{\prime}\right)+\inf \left(a, a^{\prime}\right) \sup \left(a, a^{\prime}\right) \inf \left(b, b^{\prime}\right) \sup \left(b, b^{\prime}\right)= \\
& =\sum_{(\rho, a, b),\left(\rho, a^{\prime}, b^{\prime}\right) \in \operatorname{Jord}(\psi)} \inf \left(a, a^{\prime}\right)\left(1+\sup \left(a, a^{\prime}\right)\right) \inf \left(b, b^{\prime}\right)\left(1+\sup \left(b, b^{\prime}\right)\right) .
\end{aligned}
$$

C'est le résultat annoncé.

\subsection{Comparaison avec notre normalisation de l'action de $\theta$}

Dans ce travail, nous avons défini une normalisation adhoc pour l'action de $\theta$. Elle s'exprime comme un mélange de $\theta_{W}$ et $\theta_{U}$; au lieu de plonger $W_{F}$ dans la première où la deuxième copie de $S L(2, \mathbb{C})$, on choisit la copie en fonction de $(\rho, a, b)$ et plus précisément en fonction du signe de $a-b$. L'ordre dans lequel on effectue les opérations est important pour les $\psi$ élémentaires même de restriction discrète à la diagonale (c'est -à-dire tel que pour tout $(\rho, a, b) \in \operatorname{Jord}(\psi)$, inf $(a, b)=1)$. Ensuite, nous sommes passés des $\psi$ élémentaires aux $\psi$ de restriction discrète à la diagonale sans rajouter de choix . Le choix redevient crucial quand on traite le cas des $\psi$ généraux. Il y a toutefois une astuce simple pour ne pas recommencer les calculs.

On pose $\mathcal{Z}(\psi)$ l'ensemble des couples ordonnés $\left((\rho, a, b),\left(\rho, a^{\prime}, b^{\prime}\right)\right) \in \operatorname{Jord}(\psi)$ tels que $\sup \left(b, b^{\prime}\right)$ et $\sup \left(a, a^{\prime}\right)$ sont pairs tandis que inf $\left(b, b^{\prime}\right)$ et inf $\left(a, a^{\prime}\right)$ sont impairs; on remarque immédiatement que ces conditions excluent la possibilité $(\rho, a, b)=\left(\rho^{\prime}, a^{\prime}, b^{\prime}\right)$. On pose aussi

$Z_{W}(\psi):=\left\{(\rho, a, b),\left(\rho, a^{\prime}, b^{\prime}\right) \in \mathcal{Z}(\psi) ; a+a^{\prime}<b+b^{\prime}\right.$, ou $a-b=a^{\prime}-b^{\prime} \neq 0$ et $\left.\zeta(a+b)+\zeta^{\prime}\left(a^{\prime}+b^{\prime}\right)<0\right\}$, où $\zeta$ est le signe de $a-b$ et $\zeta^{\prime}$ celui de $a^{\prime}-b^{\prime}$. On pose alors

$$
\mathcal{Z}_{U}(\psi)=\mathcal{Z}(\psi) \backslash \mathcal{Z}_{W}=
$$




$$
\begin{gathered}
\left\{(\rho, a, b),\left(\rho, a^{\prime}, b^{\prime}\right) \in \mathcal{Z}(\psi) ; a+a^{\prime}>b+b^{\prime}, \text { ou } a-b=b^{\prime}-a^{\prime}=0,\right. \\
\text { ou } \left.a-b=b^{\prime}-a^{\prime} \neq 0, \zeta(a+b)+\zeta^{\prime}\left(a^{\prime}+b^{\prime}\right) \geq 0\right\} .
\end{gathered}
$$

Ces 3 ensembles admettent donc une symétrie (celle qui échange les 2 éléments des couples) qui n'a pas de points fixes. Les cardinaux sont donc pairs.

Comme nous en aurons aussi besoin, on peut récrire ces 2 ensembles en utilisant les quadruplets $(\rho, A, B, \zeta),\left(\rho, A^{\prime}, B^{\prime}, \zeta^{\prime}\right)$ plutôt que les triplets et cela donne :

$$
\begin{gathered}
\mathcal{Z}_{W}(\psi):=\left\{(\rho, A, B, \zeta),\left(\rho, A^{\prime}, B^{\prime}, \zeta^{\prime}\right) ; \zeta B+\zeta^{\prime} B^{\prime}<0, \text { ou } B=B^{\prime} \neq 0, \zeta \zeta^{\prime}=-1, \zeta\left(A-A^{\prime}\right)<0\right\} \\
\mathcal{Z}_{U}(\psi):=\left\{(\rho, A, B, \zeta),\left(\rho, A^{\prime}, B^{\prime}, \zeta^{\prime}\right) ; \zeta B+\zeta^{\prime} B^{\prime}>0, \text { ou } B=B^{\prime}=0\right. \\
\text { ou } \left.B=B^{\prime} \neq 0, \zeta \zeta^{\prime}=-1, \zeta\left(A-A^{\prime}\right) \geq 0\right\}
\end{gathered}
$$

en effet $a-b+a^{\prime}-b^{\prime}$ vaut 2 fois $\zeta B+\zeta^{\prime} B$. Quand $\zeta B=-\zeta^{\prime} B^{\prime}$, on a soit $B=B^{\prime}=0$ soit $B B^{\prime} \neq 0$ et sous cette dernière hypothèse, nécessairement $\zeta \zeta^{\prime}=-1$. Ensuite, on écrit $\zeta(a+b)+\zeta^{\prime}\left(a^{\prime}+b^{\prime}\right)=$ $\zeta(2 A+2)+\zeta^{\prime}\left(2 A^{\prime}+2\right)=2\left(\zeta\left(A-A^{\prime}\right)\right)$ si $\zeta \zeta^{\prime}=-1$. D'où la récriture faite.

Pour $(\rho, a, b) \in \operatorname{Jord}(\psi)$, on écrit $\mathcal{Z}(\psi)_{(\rho, a, b)}, \mathcal{Z}_{W}(\psi)_{(\rho, a, b)}, \mathcal{Z}_{U}(\psi)_{(\rho, a, b)}$ l'ensemble des éléments $\left(\rho, a^{\prime}, b^{\prime}\right) \in \operatorname{Jord}(\psi)$ tels que $\left((\rho, a, b),\left(\rho, a^{\prime}, b^{\prime}\right)\right)$ soit respectivement dans $\mathcal{Z}(\psi), \mathcal{Z}_{W}(\psi)$ et $\mathcal{Z}_{U}(\psi)$. Il faut ici remarquer que les conditions de parité qui définissent $\mathcal{Z}$, entraînent que si $\left((\rho, a, b),\left(\rho, a^{\prime}, b^{\prime}\right)\right) \in$ $\mathcal{Z}$ alors $a^{\prime} \neq a$ et $b^{\prime} \neq b$. En particulier les ensembles que l'on vient de définir relativement à $(\rho, a, b)$ fixé sont des sous-ensembles de $\operatorname{Jord}\left(\psi^{\prime}\right)$ où $\psi^{\prime}$ s'obtient à partir de $\psi$ en enlevant $(\rho, a, b)$.

Théorème. L'action de $\theta$ mise sur $\pi(\psi)$ diffère de $\theta_{W}(\psi)$ par le signe $z_{W}(\psi):=(-1)^{1 / 2\left|\mathcal{Z}_{W}(\psi)\right|}$ et de $\theta_{U}(\psi)$ par le signe $z_{U}(\psi):=(-1)^{1 / 2\left|\mathcal{Z}_{U}(\psi)\right|}$.

Tenant compte du théorème de 5.5.4, il suffit de démontrer l'une des assertions. On fait d'abord les remarques suivantes. Soit $(\rho, a, b) \in \operatorname{Jor} d(\psi)$. On note $\psi^{\prime}$ le morphisme qui se déduit de $\psi$ en enlevant ce triplet.

Que ce soit pour les morphismes $\psi$ élémentaires ou pour ceux de restriction discrète à la diagonale, on a défini l'action $\theta(\psi)$ sur $\pi(\psi)$, en précisant un quadruplet $(\rho, A, B, \zeta) \in \operatorname{Jord}(\psi)$ tel que, en notant $\psi^{\prime}$ le morphisme qui se déduit de $\psi$ en enlevant ce quadruplet, l'action $\theta(\psi)$ est le prolongement canonique, utilisant l'inclusion ci-dessous, de l'action $\theta\left(\psi^{\prime},(\rho, A-1, B+1, \zeta)\right.$ si $A>B$ et $\theta\left(\psi^{\prime},(\rho, A-\right.$ $1, B-1, \zeta))$ si $A=B \neq 0$. Le premier cas est celui de $\psi$ de restriction discrète à la diagonale où l'on peut prendre n'importe quel quadruplet $(\rho, A, B, \zeta)$ du moment que $A>B$ et le deuxième cas est celui de $\psi$ élémentaire où il y a la condition $B$ minimal parmi les $\left(\rho, A^{\prime}, B^{\prime}, \zeta^{\prime}\right)$ vérifiant $B^{\prime} \neq 0$ et si $\operatorname{Jord}(\psi)$ contient à la fois $(\rho, A, B, \zeta=+)$ et $(\rho, A, B, \zeta=-)$ on prend $(\rho, A, B, \zeta=-)$. L'inclusion est, si $A>B$ :

$$
\pi(\psi) \hookrightarrow<\rho||^{\zeta B}, \cdots, \rho||^{-\zeta A}>\times \pi\left(\psi^{\prime},(\rho, A-1, B+1, \zeta) \times<\rho||^{\zeta A}, \cdots, \rho||^{-\zeta B}>;\right.
$$

et si $\psi$ est élémentaire et $B>0$

$$
\pi(\psi) \hookrightarrow \rho||^{\zeta B} \times \pi\left(\psi^{\prime},(\rho, A-1=B-1, B-1, \zeta)\right) \times \rho||^{-\zeta B} .
$$

Remarquons que dans $(1)$, si $\zeta=+,<\rho||^{\zeta B}, \cdots,\left.\rho\right|^{-\zeta A}>$ n'est autre que $\left.S t(a)\right|^{-(b-1) / 2}$ et si $\zeta=-$ cette représentation est isomorphe à $S p(b)||^{(a-1) / 2}$. D'après 5.4, si $\zeta=+($ resp. $\zeta=-)$, (1) entrelace $\theta_{W}(\psi)$ (resp. $\left.\theta_{U}(\psi)\right)$ et le prolongement canonique de $\theta_{W}\left(\psi^{\prime},(\rho, A-1, B+1, \zeta)\right.$ ) (resp. $\left.\theta_{U}\left(\psi^{\prime},(\rho, A-1, B+1, \zeta)\right)\right)$. Pour (2), si $\zeta=+$ on écrit $\left.\rho\right|^{\zeta B}$ sous la forme $\left.\rho\right|^{(a-1) / 2}$ et (2) entrelace $\theta_{U}(\psi)$ avec $\theta_{U}\left(\psi^{\prime},(\rho, A-1, B-1, \zeta)\right)$ et si $\zeta=-, \rho||^{\zeta B}=\left.\rho\right|^{-(b-1) / 2}$ et l'entrelacement est analogue en remplaçant $U$ par $W$. On aura donc démontré le théorème par récurrence pour les $\psi$ élémentaires et les $\psi$ de restriction discrète à la diagonale si l'on peut démontrer les propriétés suivantes :

(3) on suppose que $\psi$ est de restriction discrète à la diagonale. $\mathrm{Si} A>B, \mathcal{Z}_{?}(\psi)_{(\rho, A, B, \zeta)}=$ $\mathcal{Z}_{?}\left(\psi^{\prime},(\rho, A-1, B+1, \zeta)\right)_{(\rho, A-1, B+1, \zeta)}$, où $?=W($ resp $=U)$ si $\zeta=+$ (resp. - ) et en particulier est vide si $A=B+1$ (l'ensemble de droite est vide si $A=B+1$ ); 
(4) on suppose que $\psi$ est élémentaire et $B \geq 1 / 2$ (avec l'hypothèse de minimalité déjà écrite) $\mathcal{Z}_{?}(\psi)_{(\rho, A, B, \zeta)}=\mathcal{Z}_{?}\left(\psi^{\prime},(\rho, A-1, B-1, \zeta)\right)_{(\rho, A-1, B-1, \zeta)}$, où $?=U($ resp $=W)$ si $\zeta=+($ resp. -$)$ (l'ensemble de droite est vide si $B=1 / 2$ ).

Plus généralement, car on en aura besoin pour le cas général, on fixe $(\rho, A, B, \zeta)$ ou plutôt $(\rho, a, b)$; on suppose d'abord que $b \geq 2$; on remarque que $(\rho, a, b-2)$ correspond à $(\rho, A-1, B+1, \zeta)$ si $\zeta=+$ et à $(\rho, A-1, B-1, \zeta)$ si $\zeta=-$. Et on montre :

$\mathcal{Z}_{W}(\psi)_{(\rho, a, b)}$ est vide si $b=2$ et contient $\mathcal{Z}_{W}\left(\psi^{\prime},(\rho, a, b-2)\right)_{(\rho, a, b-2)}$ sinon, avec égalité sauf s'il existe $\left(\rho, A^{\prime}, B^{\prime}, \zeta^{\prime}\right) \in \operatorname{Jord}\left(\psi^{\prime}\right)$ tel que

soit $B^{\prime} \neq 0$ et $\zeta B+\zeta^{\prime} B^{\prime}=-1$ et $\zeta^{\prime}\left(A^{\prime}-A+1\right) \geq 0$,

soit $B^{\prime}=B \neq 0, \zeta \zeta^{\prime}=-$ et $\zeta\left(A-A^{\prime}\right)<0$.

Supposons d'abord que $b=2 ;$ comme $\mathcal{Z}_{W}(\psi)_{(\rho, a, b)}$ est inclus dans $\mathcal{Z}(\psi)_{(\rho, a, b)}$ les éléments $\left(\rho, a^{\prime}, b^{\prime}\right)$ de $\mathcal{Z}_{W}(\psi)_{(\rho, a, b)}$ vérifie certainement $\inf \left(b, b^{\prime}\right)$ est impair et ne peut donc valoir $b$. Ainsi $b=\sup \left(b, b^{\prime}\right)$ et $b^{\prime}=\inf \left(b, b^{\prime}\right)$ vaut nécessairement 1 . D'après les propriétés de $\mathcal{Z}_{W}$, on a $a+a^{\prime} \leq b+b^{\prime}=3$ et les conditions de parité entraînent encore $\sup \left(a, a^{\prime}\right)=2$, inf $\left(a, a^{\prime}\right)=1$. D'où $a+a^{\prime}=b+b^{\prime}$ et il faut donc $\zeta \zeta^{\prime}=-1 a-b=b^{\prime}-a^{\prime} \neq 0$. D'où encore $a=1$ et $a^{\prime}=2$ mais alors $\zeta(a+b)+\zeta^{\prime}\left(a^{\prime}+b^{\prime}\right)$ vaut 0 et n'est donc pas $<0$. D'où la première assertion.

Supposons maintenant que $b>2$. Il est clair que tout élément $\left(\rho, a^{\prime}, b^{\prime}\right) \in \mathcal{Z}_{W}\left(\psi^{\prime},(\rho, a, b-2)\right)_{(\rho, a, b-2)}$ vérifie $a+a^{\prime} \leq b-2+b^{\prime}<b+b^{\prime}$ et est donc un élément de $\mathcal{Z}_{W}(\psi)_{(\rho, a, b)}$. Réciproquement soit $\left(\rho, a^{\prime}, b^{\prime}\right) \in \mathcal{Z}_{W}(\psi)_{(\rho, a, b)}$. Si $a+a^{\prime}<b+b^{\prime}-2$, il est aussi dans $\mathcal{Z}_{W}\left(\psi^{\prime},(\rho, a, b-2)\right)_{(\rho, a, b-2)}$. Supposons maintenant que $a+a^{\prime}=b+b^{\prime}-2$; un tel élément n'est pas dans $\mathcal{Z}_{W}\left(\psi^{\prime},(\rho, a, b-2)_{(\rho, a, b-2)}\right.$ si

soit $B^{\prime}=0$ d'où nécessairement $\zeta B=-1$, mais alors $a^{\prime}=b^{\prime}, b=a+1$ et les conditions de parité sur inf $\left(a, a^{\prime}\right), \inf \left(b, b^{\prime}\right)$ et $\sup \left(a, a^{\prime}\right), \sup \left(b, b^{\prime}\right)$ ne peuvent être satisfaites car 3 de ces nombres ont même parité;

soit $B^{\prime} \neq 0$ d'où nécessairement $(a-(b-2))$ est du signe $-\zeta^{\prime}$ et, pour que cette élément ne soit pas dans $\mathcal{Z}_{W}\left(\psi^{\prime},(\rho, a, b-2)\right)_{(\rho, a, b-1)},-\zeta^{\prime}(a+b-2)+\zeta^{\prime}\left(a^{\prime}+b^{\prime}\right) \geq 0$. Cette dernière condition se récrit $-\zeta^{\prime}(A-1)+\zeta^{\prime} A^{\prime} \geq 0$ ou encore $\zeta^{\prime} A^{\prime} \geq \zeta^{\prime}(A-1)$ et la condition $a+a^{\prime}=b+b^{\prime}-2$ est $\zeta B+\zeta^{\prime} B^{\prime}=-1$.

Il reste à voir le cas où $a+a^{\prime}=b+b^{\prime}$ et alors nécessairement $B=B^{\prime} \neq 0, \zeta \zeta^{\prime}=-$ et $\zeta\left(A-A^{\prime}\right)<0$. Ce sont les conditions de l'assertion.

Utilisons les tout de suite pour démontrer (3) sous l'hypothèse $\zeta=+:$ l'hypothèse $b \geq 2$ est alors équivalente à $A \geq B+1$ les égalités se correspondant. Ainsi, si $A=B+1$, on a vu que les 2 ensembles sont vides. Supposons que $A>B+1$. Il n'existe certainement pas $\left(\rho, A^{\prime}, B^{\prime}, \zeta^{\prime}\right) \in \operatorname{Jord}\left(\psi^{\prime}\right)$ avec $B^{\prime}=B$; supposons que l'on ait $\zeta B+\zeta^{\prime} B^{\prime}=-1$ et $\zeta^{\prime} A^{\prime} \geq \zeta^{\prime}(A-1)$; comme $\zeta=+$ par hypothèse, $B+1=-\zeta^{\prime} B^{\prime}$ d'où $\zeta^{\prime}=-$ et $B^{\prime}=B+1, A \geq A^{\prime}+1$, en particulier $\left[B^{\prime}, A^{\prime}\right] \subset[B, A]$ ce qui est aussi impossible puisque dans (3), on a supposé que $\psi$ est de restriction discrète à la diagonale. Cela prouve (3) sous l'hypothèse $\zeta=+$.

On démontre aussi (4) sous les hypothèses $\zeta=-$; l'hypothèse $\zeta=-$ et le fait que $\psi$ est supposé élémentaire dans l'énoncé de $(4)$, donnent $B=(b-1) / 2$. Ainsi le fait que $b \geq 2$ est ici équivalenteà $B \geq 1 / 2$ et $b>2$ à $B \geq 1$. On a donc immédiatement l'égalité cherchée si $B=1 / 2$ puisque l'ensemble de gauche est vide lui aussi. Supposons donc que $B \geq 1$. Comme $\psi$ est élémentaire si $\left(\rho, A^{\prime}, B^{\prime}, \zeta^{\prime}\right) \in \operatorname{Jord}\left(\psi^{\prime}\right)$ vérifie $B^{\prime}=B$, il vérifie aussi $A^{\prime}=A$; il ne gêne donc pas. Supposons que $\zeta B+\zeta^{\prime} B^{\prime}=-1, B^{\prime} \neq 0$ et $\zeta^{\prime} A^{\prime} \geq \zeta^{\prime}(A-1)$; cela se traduit par $\zeta^{\prime} B^{\prime}=B-1$ d'où puisque $B^{\prime} \neq 0$ nécessairement $\zeta^{\prime}=+$ et $B^{\prime}=B-1$ ce qui contredit la minimalité de $B$. Il reste encore le cas où $B^{\prime}=0, B=1$, c'est-à-dire $\left(a^{\prime}, b^{\prime}\right)=(1,1)$ et $(a, b)=(1,3)$ mais un tel couple n'est pas dans $\mathcal{Z} \psi$ pour des conditions de parité.

Supposons que $a=2$, alors $\mathcal{Z}_{U}(\psi)_{(\rho, a, b)}=\emptyset$ sauf si $b=2$ et $(\rho, 1,1) \in \operatorname{Jord}\left(\psi^{\prime}\right)$ ou $b=1$ et $(\rho, 1,2) \in \operatorname{Jord}\left(\psi^{\prime}\right)$.

En effet, soit $\left(\rho, a^{\prime}, b^{\prime}\right) \in \mathcal{Z}_{U}(\psi)_{(\rho, a, b)}$. Les conditions de parité pour $\sup \left(a, a^{\prime}\right)$ et inf $\left(a, a^{\prime}\right)$ donnent $a=\sup \left(a, a^{\prime}\right)=2$ et $a^{\prime}=\inf \left(a, a^{\prime}\right)=1$. Comme $a+a^{\prime} \geq b+b^{\prime}$, les conditions de parité pour $\sup \left(b, b^{\prime}\right)$ et $\inf \left(b, b^{\prime}\right)$ donnent $\sup \left(b, b^{\prime}\right)=2$ et $\inf \left(b, b^{\prime}\right)=1$. D'où $\left(\rho, a^{\prime}, b^{\prime}\right)$ vaut soit $(\rho, 1,1)$ mais alors $b=2$ soit vaut $(\rho, 1,2)$ mais $b=1$. Ce sont les cas annoncés. 
Supposons maintenant que $a>2$, et montrons que $\mathcal{Z}_{U}(\psi)_{(\rho, a, b)}$ contient $\mathcal{Z}_{U}\left(\psi^{\prime},(\rho, a-2, b)\right)_{(\rho, a-2, b)}$ avec égalité sauf s'il existe $\left(\rho, A^{\prime}, B^{\prime}, \zeta^{\prime}\right) \in \operatorname{Jord}\left(\psi^{\prime}\right)$ tel que

soit $B=B^{\prime}=0$

soit $B=B^{\prime} \neq 0, \zeta \zeta^{\prime}=-1$ et $\zeta\left(A-A^{\prime}\right) \geq 0$

soit $\zeta B+\zeta^{\prime} B^{\prime}=1, B^{\prime} \neq 0$ et $\zeta^{\prime}\left(A^{\prime}+1-A\right)<0$.

Soit $\left(\rho, a^{\prime}, b^{\prime}\right) \in \mathcal{Z}_{U}\left(\psi^{\prime},(\rho, a-2, b)\right)_{(\rho, a-2, b)} ;$ nécessairement $b+b^{\prime} \leq a-2+a^{\prime}<a+a^{\prime}$ et $\left(\rho, a^{\prime}, b^{\prime}\right) \in$ $\mathcal{Z}_{U}(\psi)_{(\rho, a, b)}$. Supposons maintenant que $\left(\rho, a^{\prime}, b^{\prime}\right) \in \mathcal{Z}_{U}(\psi)_{(\rho, a, b)} ;$ si $b+b^{\prime}<a-2+a^{\prime}$, cet élément est dans $\mathcal{Z}_{U}\left(\psi^{\prime},(\rho, a-2, b)\right)_{(\rho, a-2, b)}$. Supposons que $b+b^{\prime}=a-2+a^{\prime}$; cela se retraduit en $\zeta B+\zeta^{\prime} B^{\prime}=1$. L'élément $\left(\rho, a^{\prime}, b^{\prime}\right)$ est dans $\mathcal{Z}_{U}\left(\psi^{\prime},(\rho, a-2, b)\right)$ sauf si $B^{\prime} \neq 0$ et $\zeta^{\prime \prime}(a-2+b)+\zeta^{\prime}\left(a^{\prime}+b^{\prime}\right)<0$, où $\zeta^{\prime \prime}$ est le signe de $a-2-b$ c'est-à-dire $-\zeta^{\prime}$. Cette dernière inégalité est donc $\zeta^{\prime}\left(A^{\prime}+1-A\right)<0$. Reste le cas $a+a^{\prime}=b+b^{\prime}$, c'est-à-dire $\zeta B+\zeta^{\prime} B^{\prime}=0$; mais on a aussi soit $B=B^{\prime}=0$ soit $B=B^{\prime} \neq 0$ d'où $\zeta=-\zeta^{\prime}$ et $\zeta\left(A-A^{\prime}\right) \geq 0$. Ce sont les conditions annoncées.

Utilisons-les pour compléter la démonstration de (3). Dans l'énoncé, on a comme hypothèse que inf $(a, b) \geq 2$ puisque $A>B$ et que $\psi$ est de restriction discrète à la diagonale. Comme on a déjà réglé le cas où $\zeta=+$, on suppose que $\zeta=-$ et .

Si $a=2, b=2$ on ne peut avoir aussi $(\rho, 1,1) \in \operatorname{Jord}(\psi)$ car $|2-2|=|1-1|$ et les segments $[B, A]$ et $\left[B^{\prime}, A^{\prime}\right]$ contiennent tous les deux 0 .

Si $a=2, b=1$ on ne peut avoir $a^{\prime}=1, b^{\prime}=2$ pour le même argument.

Or $a=2$ et équivalent à $A=B+1$ et ainsi si $a=2$ les deux ensembles de (3) sont vides et on a l'assertion. On suppose donc que $a>2$ et de façon équivalente que $A>B+1$. L'hypothèse de restriction discrète à la diagonale assure que si $\left(\rho, A^{\prime}, B^{\prime}, \zeta^{\prime}\right) \in \operatorname{Jord}\left(\psi^{\prime}\right), B^{\prime} \neq B$; il faut donc voir si l'on peut avoir $\zeta B+\zeta^{\prime} B^{\prime}=1, B^{\prime} \neq 0$ et $\zeta^{\prime}\left(A^{\prime}+1-A\right)<0$. Comme $\zeta=-$, on aurait $\zeta^{\prime} B^{\prime}=B+1$, en particulier $\zeta^{\prime}=+$, d'où $A^{\prime}+1<A$; d'où encore $\left[B^{\prime}, A^{\prime}\right] \subset[B, A]$ ce qui est exclu. D'où (3).

Complétons maintenant la démonstration de $(4)$; ici $\psi$ est élémentaire et $\zeta=+$. D'où $B=(a-1) / 2$. Supposons d'abord que $a=2$; nécessairement $b=1$. Si $(\rho, 1,2) \in \operatorname{Jord}\left(\psi^{\prime}\right)$ cela contredirait le choix minimal fait pour $(\rho, a, b)$; précisément quand $(\rho, c, 1)$ et $(\rho, 1, c)$ sont dans $\operatorname{Jord}(\psi)$ on choisit $(\rho, 1, c)$ et non $(\rho, c, 1)$. Supposons maintenant que $a>2$ d'où $B \geq 1$. Soit $\left(\rho, A^{\prime}, B^{\prime}, \zeta^{\prime}\right) \in J \operatorname{ord}\left(\psi^{\prime}\right)$ et il faut vérifier qu'il n'est pas dans les exceptions. Puisque $B \geq 1$, on n'a certainement pas $B=B^{\prime}=0$.

Considérons le cas où $B=B^{\prime} \neq 0, \zeta \zeta^{\prime}=-1$ et $\zeta\left(A-A^{\prime}\right) \geq 0$. Comme ci-dessus, on aurait $a^{\prime}=1$ et $b^{\prime}=a$ ce qui contredit le choix de $(\rho, a, b)$ comme on vient de l'expliquer.

Supposons maintenant que $\zeta B+\zeta^{\prime} B^{\prime}=1, B^{\prime} \neq 0$; d'où $\zeta^{\prime} B^{\prime}=B-1$ et la seule possibilité étant donnée l'hypothèse de minimalité sur $B$ est $B^{\prime}=0$ ce qui a été exclu. Cela termine la preuve de (4).

Nous avons donc démontré le théorème pour tous les morphismes $\psi$ élémentaires, grâce à (4) et ceux de restriction discrète à la diagonale, grâce à (3). Il reste à traiter le cas général. Ici la procédure est inverse; on part d'un $\tilde{\psi}$ de restriction discrète à la diagonale et on calcule $\pi(\psi)$ comme module de Jacquet à partir de $\pi(\tilde{\psi})$ et on a mis l'action de $\theta$ qui s'en déduit. Donc on a construit une inclusion :

$$
\pi(\tilde{\psi}) \hookrightarrow \sigma \times \pi(\psi) \times{ }^{\theta} \sigma,
$$

pour $\sigma$ convenable et $\theta(\psi)$ est tel que $\theta(\tilde{\psi})$ est le prolongement canonique de $\theta(\psi)$. Donc ici, on a le résultat pour $\theta(\tilde{\psi})$ et on veut l'obtenir pour $\theta(\psi)$. On le fait en plusieurs étapes et on se ramène à la situation suivante :

il existe $(\rho, A, B, \zeta) \in \operatorname{Jord}(\tilde{\psi})$ avec $B \geq 1$ tel que en notant $\psi^{\prime}$ le morphisme qui se déduit de $\tilde{\psi}$ en enlevant ce bloc, $\psi$ s'obtient à partir de $\psi^{\prime}$ en ajoutant le bloc $(\rho, A-1, B-1, \zeta)$. Et $(\rho, A, B, \zeta)$ a les propriétés suivantes : pour tout $\left(\rho, A^{\prime}, B^{\prime}, \zeta^{\prime}\right) \in \operatorname{Jord}\left(\psi^{\prime}\right)$ soit $B^{\prime}<B$ soit $B^{\prime}>>A$ et si $B^{\prime}=B-1$ alors $A-1 \geq A^{\prime}$ et si $B^{\prime}=B-1, A^{\prime}=A-1$ mais $\zeta \zeta^{\prime}=-$ alors $\zeta=+$.

Ces propriétés viennent des choix sur l'ordre que nous avons mis. Pour pouvoir utiliser tranquillement les calculs déjà faits sans s'embrouiller dans les notations, ou oublie la notation $\psi$ que l'on remplace par $\left(\psi^{\prime},(\rho, A-1, B-1, \zeta)\right)$.

Dans ces conditions dans $(5) \sigma=<\rho||^{\zeta B}, \cdots,\left.\rho\right|^{\zeta A}>$ c'est-à-dire si $(\rho, a, b)=(\rho, A, B, \zeta)$ si $\zeta=+$, $\sigma=S p(b)||^{(a-1) / 2}$ tandis que si $\zeta=-, \sigma=S t(a)||^{-(b-1) / 2}$ et si $\zeta=+(\rho, A-1, B-1, \zeta)=(\rho, a-2, b)$ 
tandis que si $\zeta=-,(\rho, A-1, B-1, \zeta)=(\rho, a, b-2)$. Ainsi avec 5.4, (5) entrelace $\theta_{U}(\tilde{\psi})$ (resp. $\left.\theta_{W}\right)$ et le prolongement canonique de $\theta_{U}\left(\psi^{\prime},(\rho, a-2, b)\left(\operatorname{resp} . \theta_{W}\right)\right.$ si $\zeta=+(\operatorname{resp} . \zeta=-)$.

Supposons que $\zeta=+$. Il faut donc démontrer que $\mathcal{Z}_{U}(\tilde{\psi})_{(\rho, a, b)}=\mathcal{Z}_{U}\left(\psi^{\prime},(\rho, a-2, b)\right)_{(\rho, a-2, b)}$. On a supposé que $B \geq 1$ or $(a-1) / 2 \geq B \geq 1$ d'où aussi $a>2$. Il faut donc montrer que les exceptions ne peuvent se produire. Fixons $\left(\rho, A^{\prime}, B^{\prime}, \zeta^{\prime}\right) \in \operatorname{Jord}\left(\psi^{\prime}\right)$; on suppose d'abord que $B=B^{\prime}$; or dans nos hypothèses, soit $B^{\prime}<B$ soit $B^{\prime}>>A$ ce qui est contradictoire.

On suppose maintenant que $\zeta B+\zeta^{\prime} B^{\prime}=1, B^{\prime} \neq 0$ et $\zeta^{\prime}\left(A^{\prime}+1-A\right)<0$. Ceci se retraduit en $B-1=-\zeta^{\prime} B^{\prime}, B^{\prime} \neq 0$, d'où déjà $B>1$ et $\zeta^{\prime}=-$ et encore $A^{\prime}>A-1$. Ceci a été exclu puisque $B^{\prime}=B-1$ entraîne $A^{\prime} \leq A-1$.

Supposons maintenant que $\zeta=-$. On reprend mot pour mot le début de la démonstration cidessus. Il faut ici démontrer que $\mathcal{Z}_{W}(\tilde{\psi})_{(\rho, a, b)}=\mathcal{Z}_{W}\left(\psi^{\prime},(\rho, a-2, b)\right)_{(\rho, a-2, b)}$. On a supposé que $B \geq 1$ or $(b-1) / 2 \geq B \geq 1$ d'où aussi $b>2$. Il faut donc montrer que les exceptions ne peuvent se produire. Fixons $\left(\rho, A^{\prime}, B^{\prime}, \zeta^{\prime}\right) \in \operatorname{Jord}\left(\psi^{\prime}\right)$; supposons d'abord que $B^{\prime}=B \neq 0, \zeta \zeta^{\prime}=-$ et $\zeta\left(A-A^{\prime}\right)<0$, ici c'est $B=B^{\prime}$ qui est exclu. Supposons maintenant que $\zeta^{\prime} B^{\prime} \neq 0$ et $\zeta B+\zeta^{\prime} B^{\prime}=-1$ et $\zeta^{\prime}\left(A^{\prime}-A+1\right) \geq 0$. Comme $\zeta=-$ cela donne $\zeta^{\prime} B^{\prime}=B-1$, d'où encore $B>1$ et $\zeta^{\prime}=+, B^{\prime}=B-1, A^{\prime} \geq A-1$. A priori on peut avoir $A^{\prime}=A-1$ mais alors, il faudrait $\zeta=+$; les hypothèses excluent donc aussi cette possibilité.

Cela termine la preuve du théorème.

\subsection{Signe et transfert}

Les différents choix pour l'action de $\theta$ entraînent un changement de signe dans le calcul des traces. Pour unifier les notations on pose $z(\psi)=z_{\emptyset}(\psi)$ et $\mathcal{Z}(\psi)=\mathcal{Z}_{\emptyset}(\psi)$. Aux différents signes $z_{\emptyset}(\psi), z_{W}(\psi)$, $z_{U}(\psi)$, on associe une application de $\operatorname{Jord}(\psi)$ dans $\{ \pm 1\}$, en posant simplement

$$
\epsilon_{?}(\rho, a, b):=(-1)^{\left|\mathcal{Z}_{?}(\psi)_{(\rho, a, b)}\right|} .
$$

On suppose que $\psi$ est à image dans ${ }^{L} H_{n}$ le groupe dual de $H_{n}$ (cf 4.1, une telle application s'interprète alors essentiellement comme un caractère du groupe $C_{e n t_{L_{n}}} \psi$. Le essentiellement vient du fait que si $H_{n}=S p(2 n, F)$ l'ensemble des applications de $\operatorname{Jord}(\psi)$ dans $\{ \pm 1\}$ s'identifie aux caractères du centralisateur de $\psi$ dans $O(2 n+1, F)$. Dans tous les cas, on note $z$ l'élément non trivial soit du centre de ${ }^{L} H_{n}$ (si $H_{n} \neq S p(2 n, F)$ )soit du centre de $O(2 n+1)$. Pour toute application de $\operatorname{Jord}(\psi)$ dans $\{ \pm 1\}$ identifiée comme ci-dessus en un caractère, on a :

$$
\epsilon(z)=\prod_{(\rho, a, b) \in \operatorname{Jord}(\psi)} \epsilon(\rho, a, b)
$$

où $\operatorname{Jord}(\psi)$ est vu comme un ensemble avec multiplicités.

On pose aussi

$$
c_{2, \psi}:=\psi\left(1_{W_{F}}, 1_{S L(2, \mathbb{C})}\left(\begin{array}{cc}
-1 & 0 \\
0 & -1
\end{array}\right)\right)
$$

, où $1_{W_{F}}$ et $1_{S L(2, \mathbb{C})}$ sont les unités des groupes en indice. Et on a :

Théorème. Pour? valant soit $\emptyset$, soit $W$ soit $U, \epsilon ?(z)=1$ et $\epsilon_{?}\left(c_{2, \psi}\right)=z_{?}(\psi)$.

D'après ce qui précède l'énoncé $: \epsilon_{?}(z)=\prod_{(\rho, a, b) \in \operatorname{Jord}(\psi)} \epsilon_{?}(\rho, a, b)$. Cela vaut précisément $(-1)^{T}$ où $T$ est le cardinal de $\cup_{(\rho, a, b) \in J o r d(\psi)} \mathcal{Z}_{\text {? }}(\psi)_{(\rho, a, b)} ;$ mais cet ensemble s'identifie exactement avec $\mathcal{Z}_{\text {? }}(\psi)$; un tel ensemble est de cardinal pair (cf. ??). D'où la première assertion de l'énoncé. Pour la deuxième, on a de façon très générale :

$$
\epsilon_{?}(\psi)\left(c_{2, \psi}\right)=\prod_{(\rho, a, b) \in \operatorname{Jord}(\psi) ;(-1)^{b}=1} \epsilon_{?}(\psi)(\rho, a, b)=(-1)^{T^{\prime}}
$$

où ici $T^{\prime}$ est le cardinal de $\cup_{(\rho, a, b) \in J o r d(\psi) ;(-1)^{b}=1} \mathcal{Z}_{?}(\psi)_{(\rho, a, b)}$. Or tout couple $(\rho, a, b),\left(\rho, a^{\prime}, b^{\prime}\right) \in \mathcal{Z}(\psi)$ est tel que soit $b$ soit $b^{\prime}$ est pair et l'autre est impair. Ainsi l'ensemble cherché est exactement $1 / 2\left|\mathcal{Z}_{\text {? }}(\psi)\right|$ et l'assertion est exactement la définition de $z_{?}(\psi)$. D'où le théorème. 
Remarque. On garde l'hypothèse sur l'image de $\psi$. Supposons que l'on ait construit une application de l'ensemble des caractères du centralisateur de $\psi$ dans l'ensemble des représentations de $H_{n}$ (en acceptant la représentation 0$), \epsilon \mapsto \pi(\psi, \epsilon)$ de telle sorte que pour $\pi(\psi)$ muni de l'action $\theta(\psi)$, la trace tordue de $\pi(\psi)$ soit un transfert du caractère $\sum_{\epsilon \in C e n \hat{t}_{L_{H_{n}}}} \epsilon\left(c_{2, \psi}\right) \operatorname{tr}(\pi(\psi, \epsilon))$. Alors la trace tordue de $\pi(\psi)$ muni de l'action $\theta_{W}(\psi)$ est un transfert de la distribution

$$
\sum_{\epsilon \in \operatorname{Cent}_{L_{H_{n}}}} \epsilon_{W}\left(c_{2, \psi}\right) \epsilon\left(c_{2, \psi}\right) \operatorname{tr}(\pi(\psi, \epsilon)) .
$$

Et on a une assertion analogue pour $\theta_{U}(\psi)$ et $\epsilon_{U}\left(c_{2, \psi}\right)$.

Il est alors intéressant de changer la paramétrisation des $\pi(\psi, \epsilon)$ c'est à dire de poser $\pi_{W}(\psi, \epsilon)=$ $\pi\left(\psi, \epsilon_{W}(\psi) \epsilon\right)$ d'où la trace tordue de $\pi(\psi)$ avec l'action $\theta_{W}(\psi)$ est un transfert de

$$
\sum_{\epsilon \in C e n \hat{t}_{L_{H}}} \epsilon\left(c_{2, \psi}\right) \operatorname{tr}\left(\pi_{W}(\psi, \epsilon)\right),
$$

ce qui est une jolie formule. Et la question est en plus de savoir si cette paramétrisation est compatible avec l'inclusion du paquet de Langlands ; précisément, on a déjà considéré en 5.1

$$
\psi^{2}: W_{F} \times S L(2, \mathbb{C}) \rightarrow G L(n, \mathbb{C})
$$

obtenu en composant $\psi$ avec le morphisme de $W_{F}$ dans la 2e copie de $S L(2, \mathbb{C}), w \mapsto\left(\begin{array}{cc}|w|^{1 / 2} & 0 \\ 0 & |w|^{-1 / 2}\end{array}\right)$. A $\psi^{2}$ correspond un paquet de Langlands, en général conjecturalement. Notons $\Pi_{L}(\psi)$ cet ensemble de représentations; il est paramétré par les caractères du centralisateur de $\psi_{\text {imp }}^{2}$ (voir 5.2) dans un Levi de ${ }^{L} H_{n}$. Il n'est pas a priori clair que $\Pi_{L}(\psi)$ soit inclus dans l'ensemble $\{\pi(\psi, \epsilon)\}$ où $\epsilon$ parcourt l'ensemble des caractères du centralisateur de $\psi$. Ceci résulte très vraisemblablement du transfert de $\pi(\psi)$ (quand celui-ci est établi) admettons le ici pour poser le problème. On doit encore remarquer que $\operatorname{Cent}_{L_{n}}(\psi)$ s'envoie naturellement dans le $\operatorname{Cent}\left(\psi_{i m p}^{2}\right)$ et la question est alors de savoir si pour $\eta$ un caractère du centralisateur de $\psi_{i m p}^{2}$ et $\epsilon$ un caractère de $\operatorname{Cent}_{L_{H_{n}}}(\psi)$ tel que $\pi_{W}(\psi, \epsilon)$ contienne la représentation de $\Pi_{L}(\psi)$ associée à $\eta, \epsilon$ serait la restriction de $\eta$ à $C e n t_{L_{H}}(\psi)$. On peut travailler de la même façon, en remplaçant $W$ par $U$ et poser la même question en utilisant $\psi_{i m p}^{1}$ de 5.3. Sur les exemples calculés les réponses sont partout oui et on reviendra ultérieurement sur ces questions.

\section{Références}

[1] Arthur J. : Unipotent automorphic representations : conjectures in Orbites unipotentes et représentations II, Astérisque 171-172, 1989, pp. 13-72

[2] Arthur J. : On local character relations, Selecta Math. 2, No 4 (1996), 501-579

[3] Arthur J. : A stable trace formula I. General expansions, JIMJ 1 (2002), 175-277

[4] Arthur J. : A stable trace formula II. Global descent, Invent. Math 143 (2001), 157-220

[5] Arthur J. : A stable trace formula III. Proof of the main theorems, Annals of Math. 158 (2003), 769-873

[6] Arthur J. : An Introduction to the Trace Formula prépublication 2005

[7] Aubert A.-M. : Dualité dans le groupe de Grothendieck de la catégorie des représentations lisses de longueur finie d'un groupe réductif p-adique, TAMS, 347, 1995, pp. 2179-2189 avec l'erratum publié dans TAMS, 348, 1996, pp. 4687-4690

[8] Bernstein I. N., Zelevinsky A. V. : Induced Representations of Reductive p-adic groups 1 Ann de l'ENS, 10, 1977, pp. 147-185 
[9] Harris, M.; TAYlor, R. : The geometry and cohomology of some simple Shimura varieties,Annals of Math Studies, 151, Princeton Univ. Press, 2001

[10] Henniart, G. : Une preuve simple des conjectures de Langlands pour $G L_{n}$ sur un corps padique,Invent. Math., 139, 2000, pp. 439-455

[11] Kottwitz R., Shelstad D. : Foundations of twisted endoscopy, Astérisque 2555, 1999

[12] Labesse J.-P. : Stable twisted trace formula : elliptic terms, JIMJ 3, 2004, pp. 473-530

[13] Lusztig G. : Classification of Unipotent Representations of Simple p-adic Groups, II,arXiv :math. RT/0111248, 2001et Int. Math. Res. Not., 1995, pp. 517-589

[14] Megalin C. : Sur certains paquets d'Arthur et involution d'Aubert-Schneider-Stuhler généralisée prépublication

[15] Megalin C. : Paquets d'Arthur discrets pour un groupe classique p-adique Xarchiv dec 2004

[16] Megalin C. : Paquets d'Arthur pour les groupes classiques p-adiques; point de vue combinatoireen préparation

[17] Megulin C. : Classification des séries discrètes pour certains groupes classiques p-adiques, prépublication 2005

[18] Megalin C. : Classification des séries discrètes : paramètre de Langlands et exhaustivité,JEMS, 4, 143-200, 2002

[19] Moeglin C., Tadic M. : Construction des séries discrètes pour des groupes classiques p-adiques (en anglais),JAMS, 15, 715-786, 2002

[20] Moeglin C. : Points de réductibilité pour les induites de cuspidales, Journal of Algebra 268, 2003

[21] Meeglin C., Waldspurger J.-L. : Le spectre résiduel de GL(n) Ann. de l'ENS 22, 1989, pp. 605-674

[22] Schneider M., Stuhler U. : Representation theory and sheaves on the Bruhat-Tits building Publ. Math. IHES 85, 1997, pp. 97-191

[23] Shahidi F. : Local coefficients and intertwining operators for GL(n), Compositio Math, 48, 1983, pp. 271-295

[24] Silberger, A. : Special representations of reductive p-adic groups are not integrable,Ann. of Math., 111, 1980, pp. 571-587

[25] Waldspurger J.-L. : La formule de Plancherel d'après Harish-Chandra JIMJ, vol2, fasc 2, 2003

[26] Waldspurger J.-L. : Le groupe $G L_{N}$ tordu, sur un corps p-adique ,1e partie, prépublication 2005

[27] Waldspurger J.-L. : Le groupe $G L_{N}$ tordu, sur un corps p-adique ,2e partie, prépublication 2005

[28] Zelevinsky A. V. : Induced Representations of Reductive p-adic groups II Ann de l'ENS, 13, 1980, pp. 165-210

moeglin@math.jussieu.fr; waldspur@math.jussieu.fr 